\title{
Electrochemical Umpolung C-H Functionalization of Oxindoles
}

\author{
Supporting Information
}

Miryam Pastor, ${ }^{\dagger}, \star$ Marie Vayer, ${ }^{\dagger}, \star$ Harald Weinstabl $^{\S}$ and Nuno Maulide $*, \dagger$

${ }^{+}$Christian Doppler Laboratory for Entropy-Oriented Drug Design, Institute of Organic Chemistry, University of Vienna, Währinger Strasse 38, 1090 Vienna, Austria and ${ }^{\S}$ Doktor-Boehringer-Gasse 5-11, 1120 Vienna, Austria.

\$ These authors contributed equally.

Table of Contents

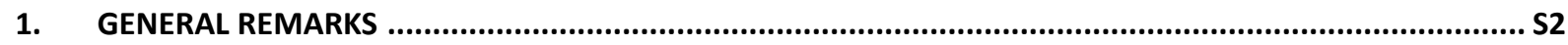

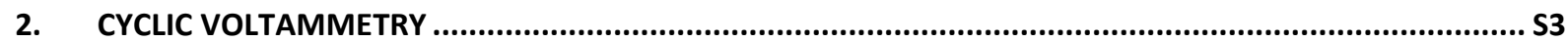

3. OPTIMIZATION OF REACTION PARAMETERS FOR ELECTROCHEMICAL FORMATION OF 3,3-

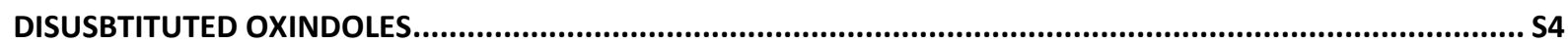

4. GENERAL PROCEDURE FOR ELECTROCHEMICAL FORMATION OF 3,3-DISUSBTITUTED OXINDOLES ....... S6

5. EXPERIMENTAL PROCEDURES AND CHARACTERIZATION DATA OF THE STARTING MATERIALS 1C-S, 3A-F

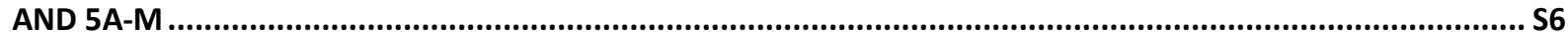

6. CHARACTERIZATION DATA OF 3,3-SUBSTITUTED OXINDOLES 2A-S, 4A-F, 6A-C AND 7A-I.................. S25

7. PROCEDURES AND CHARACTERIZATION DATA OF 3,3-SUBSTITUTED OXINDOLES 8 AND 9 (SCHEME 3) S43

8. ADDITIONAL EXPERIMENTS WITH DIFFERENT TYPE OF ALKENES ................................................ 445

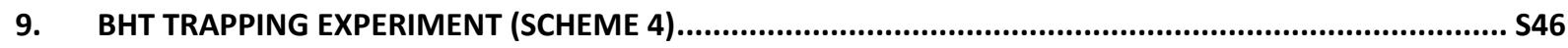

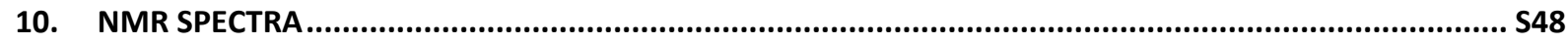

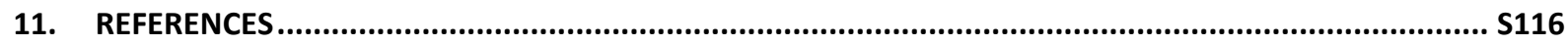




\section{General Remarks}

All solvents were distilled from appropriate drying agents prior to use or directly taken from commercial sealed bottles under an atmosphere of argon. All reagents were used as received from commercial suppliers (Alfa Aesar, Sigma Aldrich or TCI) unless otherwise stated. Reaction progress was monitored by thin layer chromatography (TLC) performed on aluminum plates coated with silica gel $\mathrm{F}_{254}$ with $0.2 \mathrm{~mm}$ thickness. Chromatograms were visualized by fluorescence quenching with UV light at $254 \mathrm{~nm}$ and/or by staining using vanillin. Flash column chromatography was performed using silica gel 60 (230-400 mesh, Merck and co.). Yields refer to chromatographically and spectroscopically pure compounds. ${ }^{1} \mathrm{H} \mathrm{NMR},{ }^{13} \mathrm{C}$ NMR and ${ }^{19} \mathrm{~F}$ NMR spectra were recorded using a Bruker AV-400 and AV-600 spectrometer at $300 \mathrm{~K} .{ }^{1} \mathrm{H}$ NMR chemical shifts are reported in ppm using residual solvent peak as reference $\left(\mathrm{CDCl}_{3}: \delta=7.26 \mathrm{ppm}\right.$ or DMSO-d $\left.6: \delta=2.50 \mathrm{ppm}\right)$. Data for ${ }^{1} \mathrm{H}$ NMR are presented as follows: chemical shift $\delta(\mathrm{ppm})$, multiplicity ( $\mathrm{s}=$ singlet, $\mathrm{d}=$ doublet, $\mathrm{t}=$ triplet, $\mathrm{m}=$ multiplet, br $=$ broad), coupling constant $J(\mathrm{~Hz})$ and integration; ${ }^{13} \mathrm{C}$ NMR spectra were recorded at 100 or $150 \mathrm{MHz}$ using broadband proton decoupling and chemical shifts are reported in ppm using residual solvent peaks as reference $\left(\mathrm{CDCl}_{3}: \delta=77.16 \mathrm{ppm}\right.$ or DMSO-d 6 : $\left.\delta=39.52 \mathrm{ppm}\right)$. Multiplicity was defined by recorded a ${ }^{13} \mathrm{C}$ NMR spectra using the attached proton test (APT). Neat infra-red spectra were recorded using a Brucker Vertex 70 FT-IR spectrometer. Wavenumbers are reported in $\mathrm{cm}^{-1}$. Mass spectra were obtained using a Finnigan MAT 8200 or $(70 \mathrm{eV})$ or an Agilent $5973(70 \mathrm{eV})$ spectrometer, using electrospray ionization (ESI) and a maXis UHR-TOF analyzer.

\section{Electrolysis general information}

Electrochemical reactions were performed with ElectraSyn 2.0 package (IKA) using the constant current mode. The reactions were conducted in a $10 \mathrm{~mL}$ vial with a magnetic stir bar and a graphite-SK-50 $(5.0 \times 0.8 \times 0.2 \mathrm{~cm})$ working electrode and counter-electrode with a distance of $0.6 \mathrm{~cm}$ between the two electrodes. 


\section{Cyclic Voltammetry}

Cyclic voltammetry was recorded with Graphite-SK-50 (5.0 x $0.8 \times 0.2 \mathrm{~cm})$ working electrode, platinum counter-electrode and aqueous $\mathrm{Ag} / \mathrm{AgCl}$ reference electrode. Scan rate: $200 \mathrm{mV} / \mathrm{s}$.

Conditions: 0.1 M Et 4 NOTs in $\mathrm{MeCN}$ as solvent.

Cyclic voltammogram of 1a showing an oxidation potential of $1.8 \mathrm{~V}$.

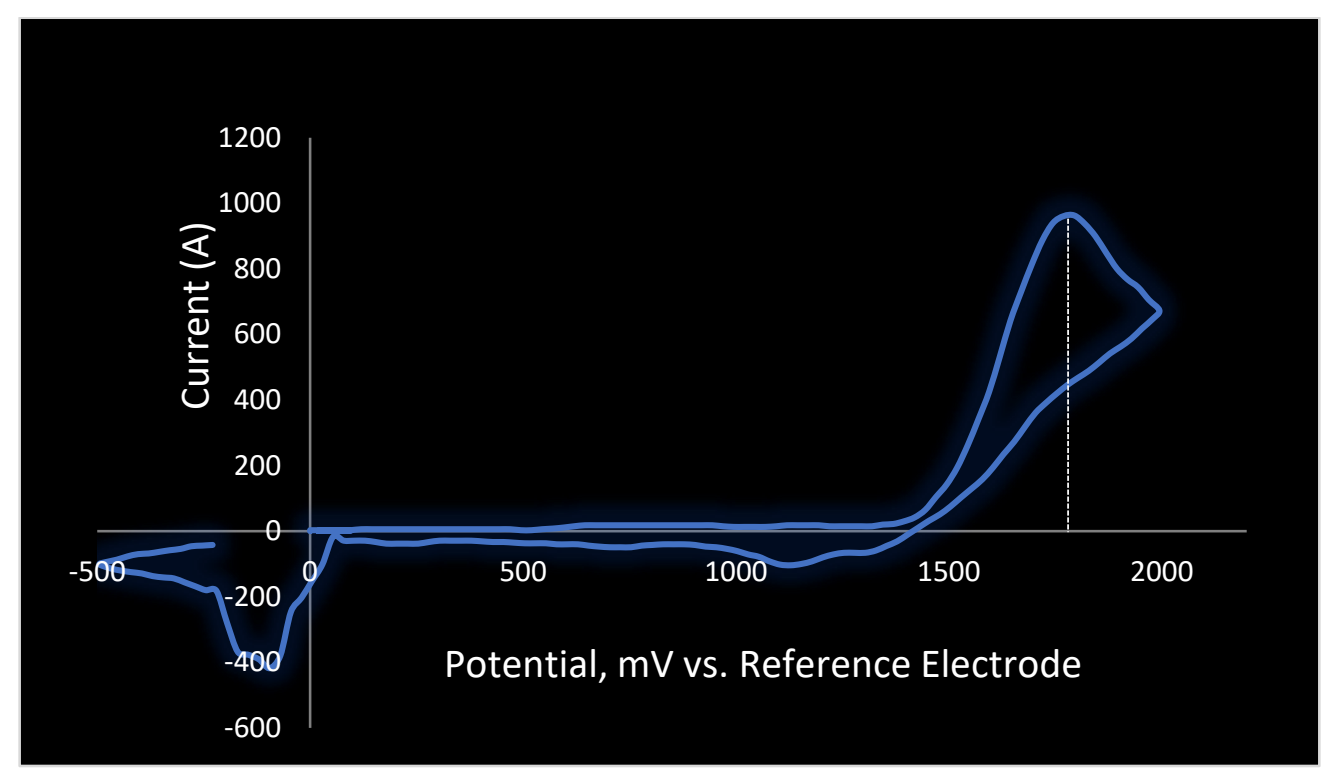

Figure S1. Cyclic voltammogram of 1a

Cyclic voltammogram of 2a showing an oxidation potential of $2.0 \mathrm{~V}$.

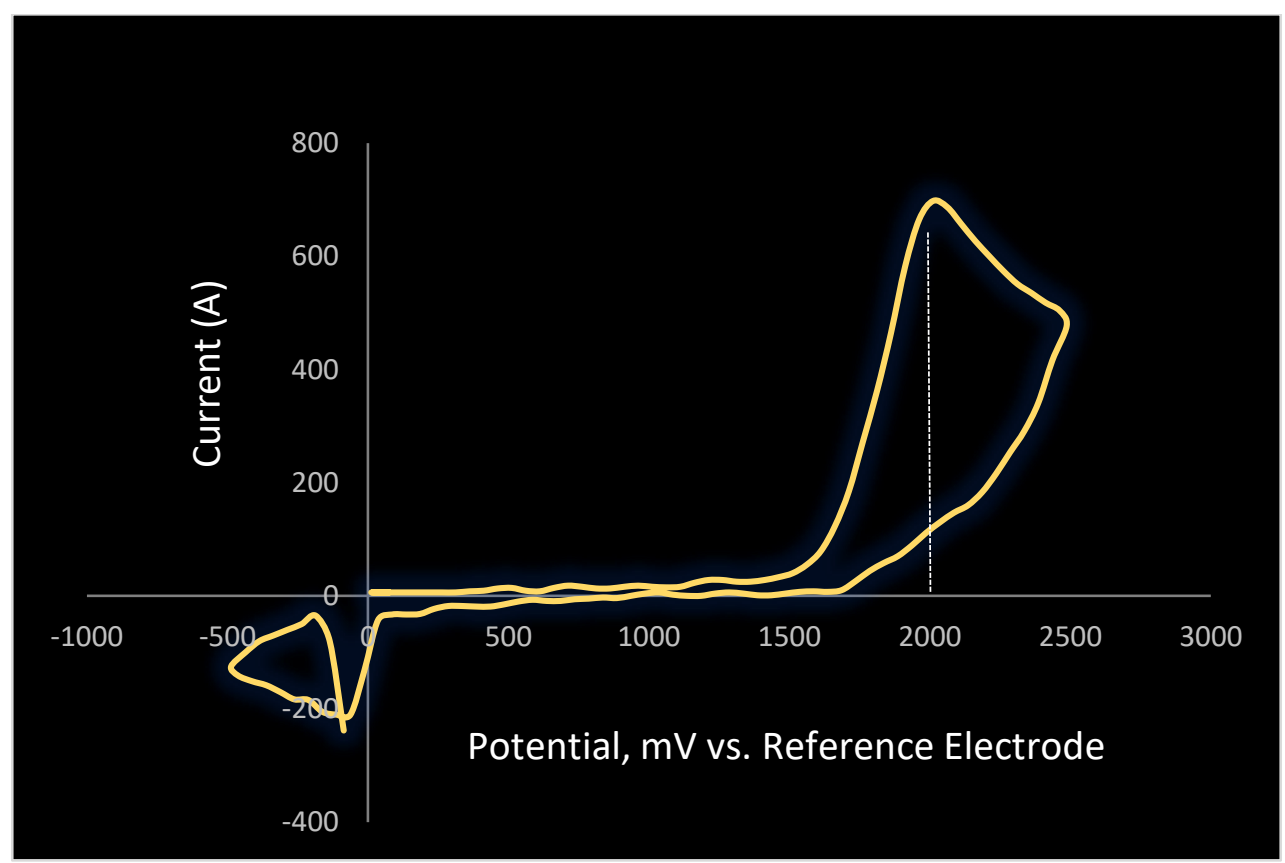

Figure S2. Cyclic voltammogram of $\mathbf{2 a}$ 


\section{Optimization of Reaction Parameters for Electrochemical Formation of 3,3-Disusbtituted Oxindoles}

All optimization reactions were carried out on $0.40 \mathrm{mmol}$ scale. The crude reaction mixture was purified by $\mathrm{FC} \mathrm{(10} \mathrm{g} \mathrm{SiO}_{2}$, heptane/ethyl acetate: 100/0 to 70/30, $\left.35 \mathrm{CV}\right)$.

Primary Evaluation of solvents (Table S1)

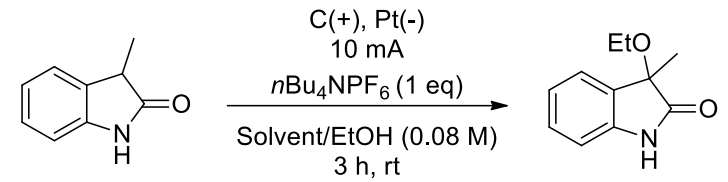

\begin{tabular}{|c|c|c|}
\hline Entry & Solvent & Yield [\%/ $^{\mathbf{a}}$ \\
\hline 1 & THF & 37 \\
\hline 2 & ACN & 40 \\
\hline 3 & DMF & ND \\
\hline 4 & DCM & 30 \\
\hline
\end{tabular}

${ }^{a}$ Isolated yield.

Evaluation of electrolytes (Table S2)

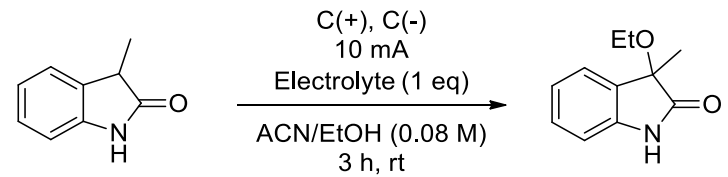

\begin{tabular}{|c|c|c|}
\hline Entry & Electrolyte & Yield [\%] $^{\mathbf{a}}$ \\
\hline 1 & $\mathrm{Et}_{4} \mathrm{NOTs}$ & $57(72 \%$ brsm $)$ \\
\hline 2 & $n \mathrm{Bu}_{4} \mathrm{NOTs}$ & 50 \\
\hline 3 & $n \mathrm{Bu}_{4} \mathrm{NPF}_{6}$ & 40 \\
\hline 4 & $n \mathrm{Bu}_{4} \mathrm{NClO}_{4}$ & 22 \\
\hline 5 & $n \mathrm{Bu}_{4} \mathrm{NOAc}_{4}$ & $\mathrm{ND}$ \\
\hline 6 & $n \mathrm{Bu}_{4} \mathrm{NBr}$ & 10 \\
\hline 7 & $\mathrm{PPTS}$ & 47 \\
\hline 8 & $\mathrm{NaPF}_{6}$ & 39 \\
\hline 9 & $\mathrm{AgPF}_{6}$ & 27 \\
\hline 10 & $\mathrm{KBF}_{4}$ & $\mathrm{ND}$ \\
\hline
\end{tabular}

${ }^{a}$ Isolated yield. 


\section{Evaluation of additives (Table S3)}

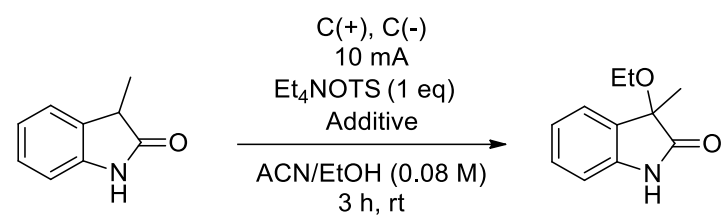

\begin{tabular}{|c|c|c|}
\hline Entry & Additive & Yield $[\%]^{\mathrm{a}}$ \\
\hline 1 & ABNO (10 mol\%) & 52 \\
\hline 2 & KetoABNO (10 mol\%) & ND \\
\hline 3 & Quinuclidine (20 mol\%) & 43 \\
\hline 4 & DDQ (20 mol\%) & 17 \\
\hline 5 & $\mathrm{DABCO}(20 \mathrm{~mol} \%)$ & 35 \\
\hline 6 & N-Hydroxytetrachlorophthalimide (10 mol\%) & 52 \\
\hline 7 & $\operatorname{HOBt}(10 \mathrm{~mol} \%)$ & 43 \\
\hline 8 & 2,4,5-Triphenylimidazole (10 mol\%) & ND \\
\hline 9 & $\operatorname{AgPF}_{6}(1 \mathrm{eq})$ & 32 \\
\hline 10 & $\operatorname{AgOTf}(1$ eq $)$ & 41 \\
\hline 11 & $\operatorname{AgNTf}_{2}(1 \mathrm{eq})$ & 38 \\
\hline 12 & $\mathrm{AgClO}_{4}(1 \mathrm{eq})$ & 37 \\
\hline 13 & $\mathrm{AgSbF}_{6}(1 \mathrm{eq})$ & 45 \\
\hline 14 & $\mathrm{AgBF}_{4}(1 \mathrm{eq})$ & 48 \\
\hline 15 & TFA $(0.5$ eq $)$ & 33 \\
\hline 16 & $\mathrm{AcOH}(0.5 \mathrm{eq})$ & 35 \\
\hline 17 & $\mathrm{AcOH}(1 \mathrm{eq})$ & 46 \\
\hline 18 & $\mathrm{~K}_{2} \mathrm{CO}_{3}(1 \mathrm{eq})$ & 23 \\
\hline 19 & 2,6-Lutidine (1 eq) & 22 \\
\hline
\end{tabular}

${ }^{\mathrm{a}}$ Isolated yield. 


\section{General Procedure for Electrochemical Formation of 3,3-Disusbtituted Oxindoles}

General procedure (A) to access 3,3-disusbtituted oxindoles

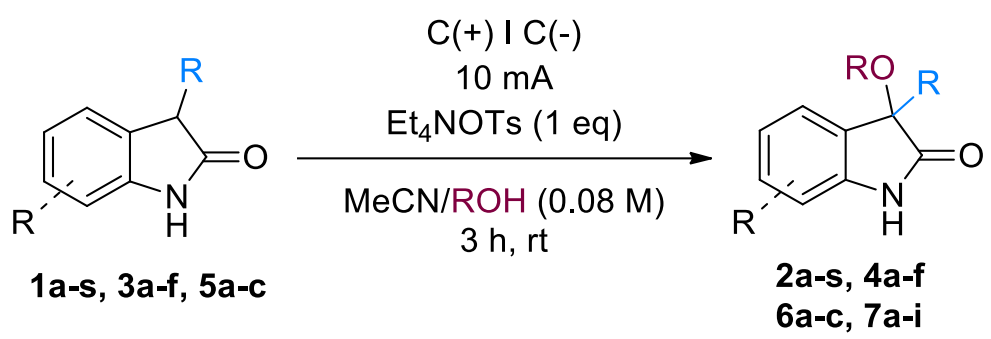

With no precautions to exclude air or moisture, the ElectraSyn vial $(10 \mathrm{~mL})$ was charged with 3-susbtituted indolin-2-one 1a-s, 3a-f or 5a-c (0.40 mmol, 1.0 equiv.), Et ${ }_{4} \mathrm{NOTs}(121.0 \mathrm{mg}$, $0.40 \mathrm{mmol}, 1.0$ equiv.), $\mathrm{ROH}(2.5 \mathrm{~mL})$ and $\mathrm{MeCN}(2.5 \mathrm{~mL})$. The ElectraSyn vial cap equipped with anode (graphite) and cathode (graphite) were inserted into the mixture. The reaction mixture was electrolyzed at a constant current of $10 \mathrm{~mA}$ for $3 \mathrm{~h}$. The ElectraSyn vial cap was removed, and electrodes were rinsed with DCM $(2.0 \mathrm{~mL})$, which was combined with the crude mixture. Then, the crude mixture was concentrated under reduced pressure and purified by FC over silica gel (heptane/ethyl acetate, 100/0 to 50/50, gradient) to furnish the desired products 2a-s, 4a-f, 6a-c or 7a-i.

\section{Experimental Procedures and Characterization Data of the Starting Materials 1c-s, 3a-f and 5a-m}

General procedure (B) to access 3-hydroxysusbtituted oxindoles (S1l-s, S3a-f, S5b)

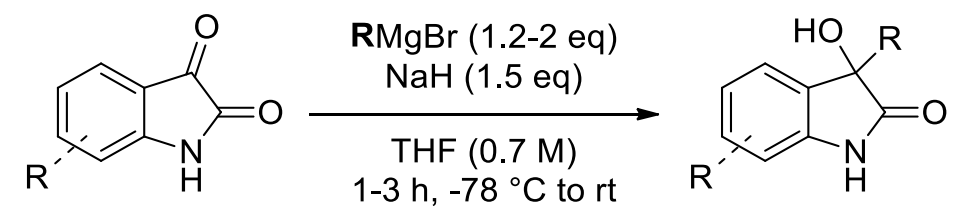

To a solution of isatin derivate (1.0 equiv.) in THF $(0.7 \mathrm{M}), \mathrm{NaH}$ (1.5 equiv.) was added at $-78{ }^{\circ} \mathrm{C}$. The mixture was stirred for $30 \mathrm{~min}$. Then, the Grignard reagent (1.2-2.0 equiv., commercially available or previously prepared) was added dropwise at this temperature. The reaction was warmed up to $\mathrm{rt}$ and stirred for $3 \mathrm{~h}$. The mixture was quenched with $\mathrm{NH}_{4} \mathrm{Cl}$, extracted with ethyl acetate, washed with $\mathrm{H}_{2} \mathrm{O}$, dried over $\mathrm{MgSO}_{4}$ and concentrated in vacuo. DCM was added to the solids obtained. Solids were filtered off and the resulting filtrate was dried in vacuo to afford the desired substrate S1l-s, S3a-f and S5b. 


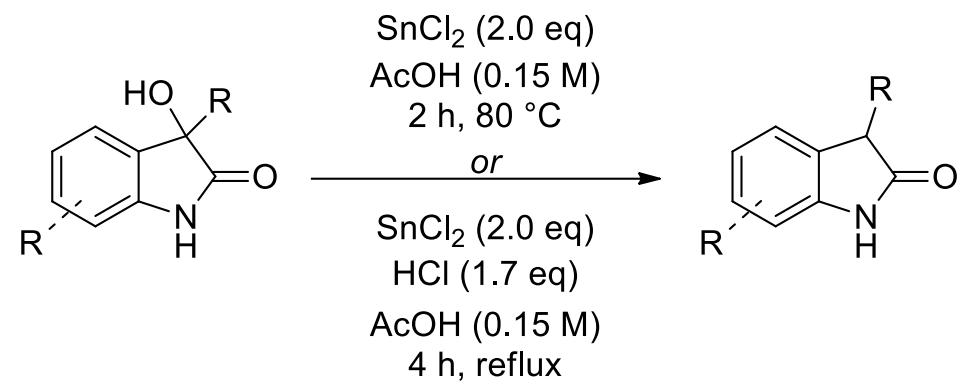

Following a modified reported procedure, ${ }^{1} 3$-hydroxysubstituted oxindole (1.0 equiv.) was dissolved in acetic acid $(0.15 \mathrm{M})$ and $\mathrm{SnCl}_{2}(2.0$ equiv.) was added in one portion. The reaction mixture was stirred at $80{ }^{\circ} \mathrm{C}$ in an oil bath for $2 \mathrm{~h}$ and then cooled to $\mathrm{rt}$ and concentrated in vacuo. The product was extracted with ethyl acetate and the organic layer was washed with $\mathrm{NaHCO}_{3}$, dried over $\mathrm{MgSO}_{4}$, and then concentrated to afford 1l-s, 3a-f and 5b. Compounds where purified by FC over silica gel (heptane/ethyl acetate, 100/0 to 70/30, gradient) to furnish the desired products.

\section{3-Isopropylindolin-2-one 1c}

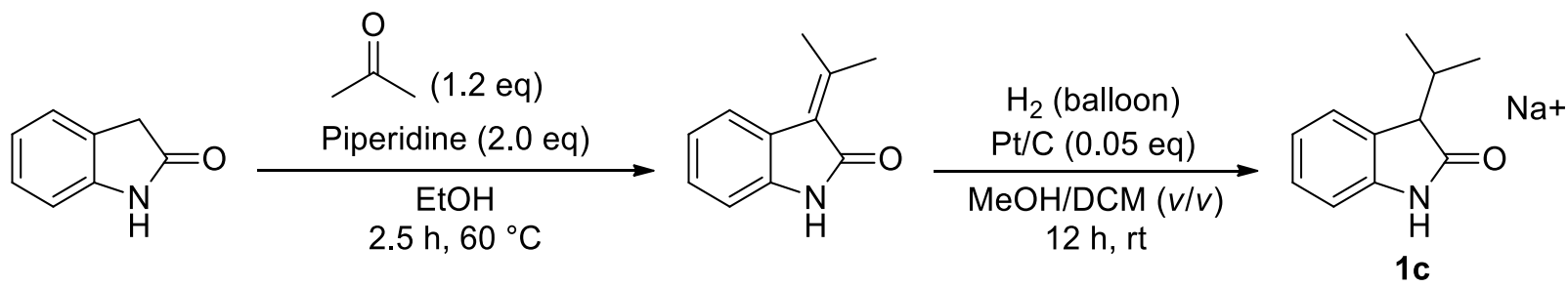

To a solution of oxindole ( $2.0 \mathrm{~g}, 15.0 \mathrm{mmol}, 1.0$ equiv.) in $\mathrm{EtOH}(15.0 \mathrm{~mL}, 0.10 \mathrm{M})$ were added acetone (1.34 mL, $18.0 \mathrm{mmol}, 1.2$ equiv.) and piperidine $(2.97 \mathrm{~mL}, 30.0 \mathrm{mmol}, 2.0$ equiv.). The reaction was then heated at reflux in an oil bath for $2.5 \mathrm{~h}$. After cooling to $0{ }^{\circ} \mathrm{C}$, the orangecolored precipitate formed was filtered, washed with cold EtOH and dried under vacuum. 3(Propan-2-ylidene)indolin-2-one is obtained as a yellow solid (1.3 g, $7.51 \mathrm{mmol}, 50 \%)$ and used in next step without further purification.

3-(Propan-2-ylidene)indolin-2-one (1.30 g, $7.51 \mathrm{mmol}, 1.0$ equiv.) was dissolved in a mixture of $\mathrm{MeOH} / \mathrm{DCM}(50 \mathrm{~mL}, v / \mathrm{v})$. Pt/C (73.2 mg, $0.380 \mathrm{mmol}, 0.05$ equiv.) was added to this solution, and the resulting mixture was stirred under hydrogen atmosphere (balloon) for $12 \mathrm{~h}$ at rt. The reaction mixture was then passed through celite, washed with DCM and concentrated in vacuo to afford $1 \mathrm{c}$ as a pale-yellow solid $(725 \mathrm{mg}, 4.14 \mathrm{mmol}, 55 \%)$.

${ }^{1} \mathbf{H}$ NMR (600 MHz, CDCl 3$): \delta 8.83$ (br s, $\left.1 \mathrm{H}\right), 7.25(\mathrm{~d}, J=7.2 \mathrm{~Hz}, 1 \mathrm{H}), 7.21(\mathrm{t}, J=7.7 \mathrm{~Hz}$, $1 \mathrm{H}), 7.01(\mathrm{td}, J=7.7,0.7 \mathrm{~Hz}, 1 \mathrm{H}), 6.90(\mathrm{~d}, J=7.7 \mathrm{~Hz}, 1 \mathrm{H}), 3.40(\mathrm{~d}, J=3.6 \mathrm{~Hz}, 1 \mathrm{H}), 2.51$ (dtd, $J=13.8,6.8,3.6 \mathrm{~Hz}, 1 \mathrm{H}), 1.13(\mathrm{~d}, J=6.8 \mathrm{~Hz}, 3 \mathrm{H}), 0.92(\mathrm{~d}, J=6.8 \mathrm{~Hz}, 3 \mathrm{H}) .{ }^{13} \mathbf{C}\left\{{ }^{1} \mathbf{H}\right\} \mathbf{~ N M R}$ (150 MHz, CDCl $)$ : $\delta 180.2(\mathrm{C}=\mathrm{O}), 142.2(\mathrm{C}), 128.5(\mathrm{C}), 127.9(\mathrm{CH}), 124.8(\mathrm{CH}), 122.2(\mathrm{CH})$, $109.7(\mathrm{CH}), 52.3(\mathrm{CH}), 30.9(\mathrm{CH}), 20.0\left(\mathrm{CH}_{3}\right), 18.1\left(\mathrm{CH}_{3}\right)$. HRMS $\left(\mathbf{E S I}^{+}\right): \mathrm{m} / z$ calcd. for $\mathrm{C}_{11} \mathrm{H}_{13} \mathrm{NONa}[\mathrm{M}+\mathrm{Na}]^{+} 198.0889$, found 198.0890 . Spectral and physical data are in accordance with literature. $^{2}$ 


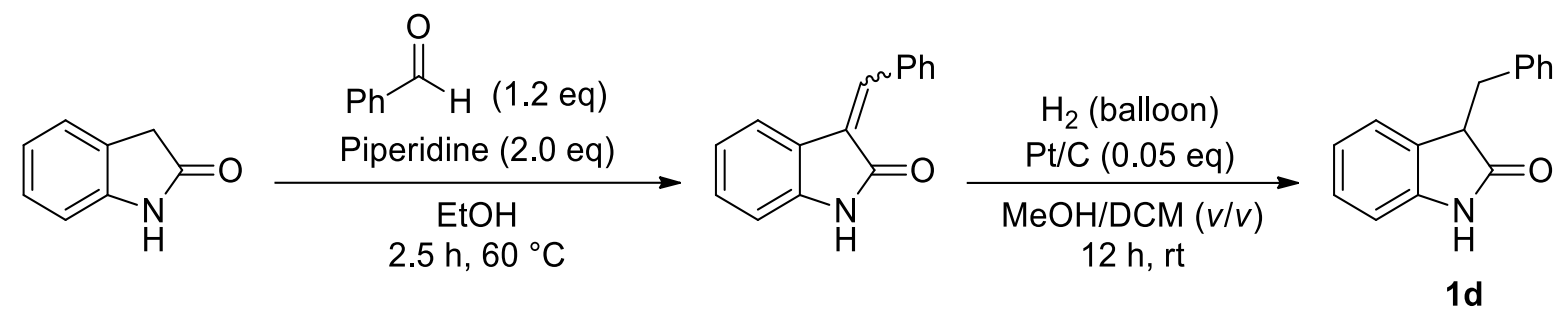

To a solution of oxindole $(500 \mathrm{mg}, 3.76 \mathrm{mmol}, 1.0$ equiv.) in EtOH $(4.0 \mathrm{~mL}, 0.10 \mathrm{M})$ were added benzaldehyde $(0.458 \mathrm{~mL}, 4.51 \mathrm{mmol}, 1.2$ equiv. $)$ and piperidine $(0.742 \mathrm{~mL}, 7.51 \mathrm{mmol}$, 2.0 equiv.). The reaction was then heated to reflux in an oil bath for $2.5 \mathrm{~h}$. After cooling to $0{ }^{\circ} \mathrm{C}$, the orange-colored precipitate formed was filtered, washed with cold $\mathrm{EtOH}$ and dried under vacuum. 3-Benzylideneindolin-2-one is obtained as a yellow solid (415 mg, $1.88 \mathrm{mmol}, 50 \%$ ) and used in next step without further purification.

Benzylideneindolin-2-one (415 g, $1.88 \mathrm{mmol}, 1.0$ equiv.) was dissolved in a mixture of $\mathrm{MeOH} / \mathrm{DCM}(16 \mathrm{~mL}, v / v)$. Pt/C (18.0 mg, $0.090 \mathrm{mmol}, 0.05$ equiv.) was added to this solution, and the resulting mixture was stirred under hydrogen atmosphere (balloon) for $12 \mathrm{~h}$ at $\mathrm{rt}$. The reaction mixture was then filtered through celite, washed with DCM and concentrated in vacuo to afford $\mathbf{1 d}$ as a pale-yellow solid (419 $\mathrm{mg}, 1.88 \mathrm{mmol}$, quant.).

${ }^{1}$ H NMR (400 MHz, CDCl3): $\delta 8.80$ (brs, 1H), 7.20-7.13 (m, 3H), 7.12-7.05 (m, 3H), $6.81(\mathrm{t}$, $J=7.5 \mathrm{~Hz}, 1 \mathrm{H}), 6.77(\mathrm{~d}, J=7.8 \mathrm{~Hz}, 1 \mathrm{H}), 6.66(\mathrm{~d}, J=7.5 \mathrm{~Hz}, 1 \mathrm{H}), 3.67(\mathrm{dd}, J=9.3,4.6 \mathrm{~Hz}$, 1H), $3.42(\mathrm{dd}, J=13.6,4.6 \mathrm{~Hz}, 1 \mathrm{H}), 2.86(\mathrm{dd}, J=13.6,9.3 \mathrm{~Hz}, 1 \mathrm{H}) .{ }^{13} \mathbf{C}\left\{{ }^{1} \mathbf{H}\right\} \mathbf{N M R}(\mathbf{1 0 0} \mathbf{~ M H z}$, CDCl3): $\delta 179.9(\mathrm{C}=\mathrm{O}), 141.6(\mathrm{C}), 137.9(\mathrm{C}), 129.6(2 \mathrm{CH}), 129.1(\mathrm{C}), 128.5(2 \mathrm{CH}), 128.1$ $(\mathrm{CH}), 126.8(\mathrm{CH}), 125.0(\mathrm{CH}), 122.1(\mathrm{CH}), 109.9(\mathrm{CH}), 47.7(\mathrm{CH}), 36.7\left(\mathrm{CH}_{2}\right)$. HRMS (ESI $\left.{ }^{+}\right)$: $\mathrm{m} / \mathrm{z}$ calcd. for $\mathrm{C}_{15} \mathrm{H}_{13} \mathrm{NONa}[\mathrm{M}+\mathrm{Na}]^{+} 246.0889$, found 246.0890 . Spectral and physical data are in accordance with literature. ${ }^{2}$

\section{3-Allylindolin-2-one 1e}

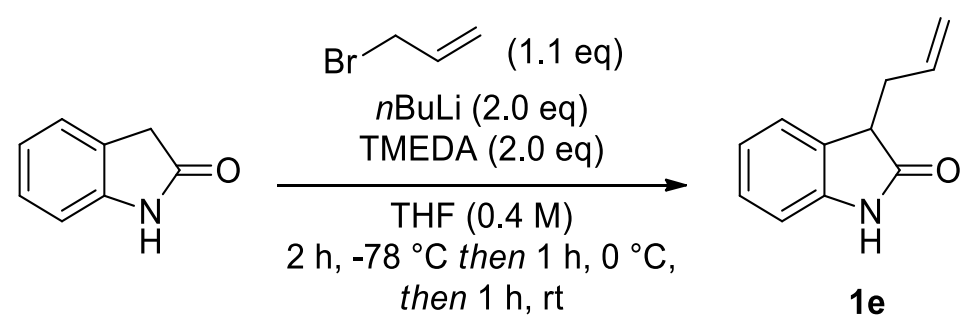

To a solution of oxindole $(500 \mathrm{mg}, 3.76 \mathrm{mmol}, 1.0$ equiv. $)$ in THF $(15.0 \mathrm{~mL})$ at $-78{ }^{\circ} \mathrm{C}$ was added $n \operatorname{BuLi}(2.5 \mathrm{M}$ in hexanes, $3.0 \mathrm{~mL}, 7.51 \mathrm{mmol}, 2.0$ equiv.) and TMEDA $(1.13 \mathrm{~mL}$, $7.51 \mathrm{mmol}, 2.0$ equiv. $)$ and the resulting solution was stirred for $2 \mathrm{~h}$. Allyl bromide $(0.357 \mathrm{~mL}$, $4.13 \mathrm{mmol}, 1.1$ equiv.) was then added dropwise and the solution was slowly warmed up to $0{ }^{\circ} \mathrm{C}$ and stirred for $1 \mathrm{~h}$ at that temperature and for $1 \mathrm{~h}$ at $\mathrm{rt}$. The reaction was then quenched with aq. $\mathrm{NH}_{4} \mathrm{Cl}$ and extracted with ethyl acetate. The combined organic layers were washed with brine, dried over $\mathrm{MgSO}_{4}$, and concentrated in vacuo. The crude was purified by FC (40 g $\mathrm{SiO}_{2}$, heptane/ethyl acetate: $100 / 0$ to $70 / 30,20 \mathrm{CV}$ ) to afford $1 \mathrm{e}$ as a white solid (305 mg, $1.76 \mathrm{mmol}, 47 \%)$. 
${ }^{1} \mathbf{H}$ NMR (400 MHz, CDCl $): \delta 8.85$ (brs, $\left.1 \mathrm{H}\right), 7.29-7.24(\mathrm{~m}, 1 \mathrm{H}), 7.21(\mathrm{t}, J=7.8 \mathrm{~Hz}, 1 \mathrm{H})$, $7.01(\mathrm{t}, J=7.5 \mathrm{~Hz}, 1 \mathrm{H}), 6.91(\mathrm{~d}, J=7.8 \mathrm{~Hz}, 1 \mathrm{H}), 5.92-5.68(\mathrm{~m}, 1 \mathrm{H}), 5.13(\mathrm{dd}, J=17.0,1.2 \mathrm{~Hz}$, $1 \mathrm{H}), 5.06(\mathrm{dd}, J=10.1,1.2 \mathrm{~Hz}, 1 \mathrm{H}), 3.54(\mathrm{dd}, J=7.2,5.2 \mathrm{~Hz}, 1 \mathrm{H}), 2.87-2.80(\mathrm{~m}, 1 \mathrm{H}), 2.65-$ $2.56(\mathrm{~m}, 1 \mathrm{H}) .{ }^{13} \mathbf{C}\left\{{ }^{1} \mathbf{H}\right\}$ NMR (100 MHz, $\left.\mathbf{C D C l}_{3}\right): \delta 180.0(\mathrm{C}=\mathrm{O}), 141.7(\mathrm{C}), 134.0(\mathrm{CH}), 129.4$ $(\mathrm{C}), 128.1(\mathrm{CH}), 124.6(\mathrm{CH}), 122.3(\mathrm{CH}), 118.2\left(\mathrm{CH}_{2}\right), 109.9(\mathrm{CH}), 45.9(\mathrm{CH}), 34.9\left(\mathrm{CH}_{2}\right)$. HRMS (ESI $\left.{ }^{+}\right): m / z$ calcd. for $\mathrm{C}_{11} \mathrm{H}_{11} \mathrm{NONa}[\mathrm{M}+\mathrm{Na}]^{+}$196.0738, found 196.0733. Spectral and physical data are in accordance with literature. ${ }^{3}$

\section{3-Cyclopentylindolin-2-one 1f}

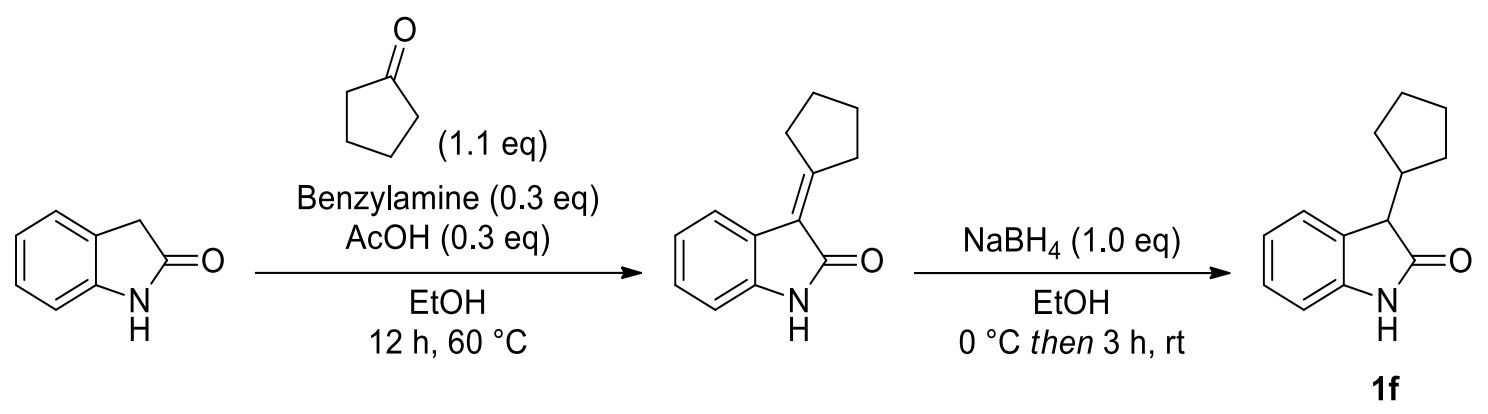

Following a procedure previously reported, ${ }^{4}$ to a $100 \mathrm{~mL}$ screw-capped pressure tube was introduced oxindole (1.0 g, $7.51 \mathrm{mmol}, 1.0$ equiv.), EtOH $(15.0 \mathrm{~mL})$ and cyclopentanone ( $0.731 \mathrm{~mL}, 8.26 \mathrm{mmol}, 1.1$ equiv.). Then, benzylamine $(0.246 \mathrm{~mL}, 1.0 \mathrm{mmol}, 0.30$ equiv.) and acetic acid $(0.129 \mathrm{~mL}, 1.0 \mathrm{mmol}, 0.30$ equiv.) were added. The tube was sealed and was heated to $60{ }^{\circ} \mathrm{C}$ in an oil bath for $12 \mathrm{~h}$. After the solvent was removed, the resulting crude mixture was dissolved in DCM and washed with $\mathrm{H}_{2} \mathrm{O}$. The aqueous phase was extracted with DCM, dried over $\mathrm{MgSO}_{4}$ and concentrated in vacuo. 3-Cyclopentylideneindolin-2-one was obtained as a brown solid (1.50 g, $7.50 \mathrm{mmol}$, quant.) and used directly in the next step without further purification.

The crude mixture was dissolved in $20 \mathrm{~mL}$ of $\mathrm{EtOH}$, and $\mathrm{NaBH}_{4}(285 \mathrm{mg}, 7.50 \mathrm{mmol}$, 1.0 equiv.) was added at $0{ }^{\circ} \mathrm{C}$ in one portion. The resulting mixture was warm up to $\mathrm{rt}$ and vigorously stirred for $3 \mathrm{~h}$. The reaction is quenched with saturated aq. $\mathrm{NH}_{4} \mathrm{Cl}$, extracted with DCM, dried over $\mathrm{MgSO}_{4}$ and concentrated in vacuo. The residue was purified by FC (40 g $\mathrm{SiO}_{2}$, heptane/ethyl acetate, 100/0 to 70/30, $\left.20 \mathrm{CV}\right)$ to afford $\mathbf{1 f}$ as a white solid $(1.12 \mathrm{~g}$, $5.56 \mathrm{mmol}, 74 \%)$.

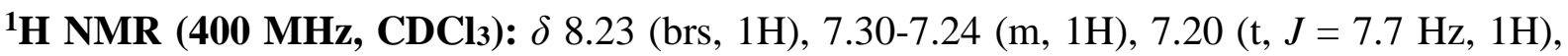
$7.00(\mathrm{td}, J=7.6,0.8 \mathrm{~Hz}, 1 \mathrm{H}), 6.87(\mathrm{~d}, J=7.8 \mathrm{~Hz}, 1 \mathrm{H}), 3.52(\mathrm{~d}, J=5.3 \mathrm{~Hz}, 1 \mathrm{H}), 2.54-2.41(\mathrm{~m}$, $1 \mathrm{H}), 1.97-1.87(\mathrm{~m}, 1 \mathrm{H}), 1.80-1.72(\mathrm{~m}, 1 \mathrm{H}), 1.68-1.49(\mathrm{~m}, 5 \mathrm{H}), 1.39-1.29(\mathrm{~m}, 1 \mathrm{H}) .{ }^{13} \mathbf{C}\left\{{ }^{1} \mathbf{H}\right\}$ NMR (100 MHz, CDCl $)$ ): $\delta 180.0(\mathrm{C}=\mathrm{O}), 141.8(\mathrm{C}), 129.3(\mathrm{C}), 127.9(\mathrm{CH}), 124.9(\mathrm{CH}), 122.2$ $(\mathrm{CH}), 109.6(\mathrm{CH}), 49.2(\mathrm{CH}), 42.0(\mathrm{CH}), 30.0\left(\mathrm{CH}_{2}\right), 28.5\left(\mathrm{CH}_{2}\right), 25.2\left(\mathrm{CH}_{2}\right), 25.2\left(\mathrm{CH}_{2}\right)$. HRMS (ESI $\left.{ }^{+}\right): m / z$ calcd. for $\mathrm{C}_{13} \mathrm{H}_{15} \mathrm{NONa}[\mathrm{M}+\mathrm{Na}]^{+} 224.1046$, found 224.1044. Spectral and physical data are in accordance with literature. ${ }^{4}$ 


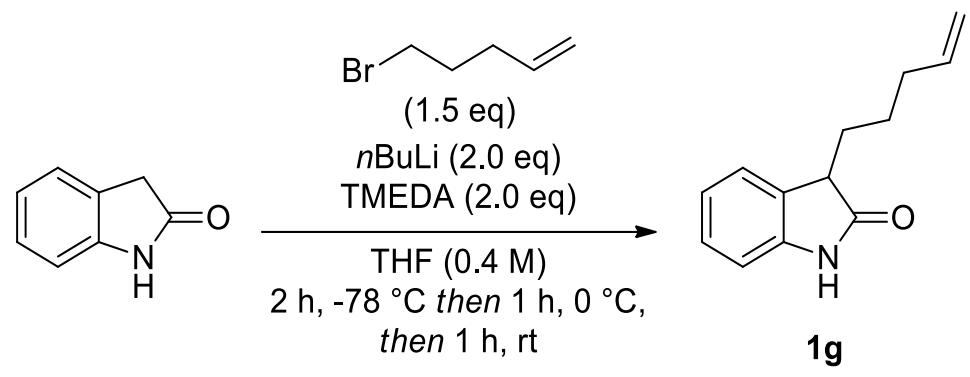

To a solution of oxindole $(2.0 \mathrm{~g}, 15.0 \mathrm{mmol}, 1.0$ equiv. $)$ in THF $(60.0 \mathrm{~mL})$ at $-78{ }^{\circ} \mathrm{C}$ was added $n \operatorname{BuLi}(2.5 \mathrm{M}$ in hexanes, $12.0 \mathrm{~mL}, 30.0 \mathrm{mmol}, 2.0$ equiv.) and TMEDA (4.51 mL, $30.0 \mathrm{mmol}$, 2.0 equiv.) and the resulting solution was stirred for $2 \mathrm{~h}$. 5-Bromo-1-pentene $(2.67 \mathrm{~mL}$, $22.5 \mathrm{mmol}, 1.1$ equiv.) was then added dropwise and the solution was slowly warmed up to $0{ }^{\circ} \mathrm{C}$ and stirred for $1 \mathrm{~h}$ at that temperature and for $1 \mathrm{~h}$ at $\mathrm{rt}$. The reaction was then quenched with aq. $\mathrm{NH}_{4} \mathrm{Cl}$ and extracted with ethyl acetate. The combined organic layers were washed with brine, dried over $\mathrm{MgSO}_{4}$, and concentrated in vacuo. The crude was purified by FC ( $80 \mathrm{~g}$ $\mathrm{SiO}_{2}$, heptane/ethyl acetate, $100 / 0$ to $70 / 30,20 \mathrm{CV}$ ) to afford $\mathbf{1 g}$ as an orange oil (326 mg, $1.62 \mathrm{mmol}, 11 \%)$.

${ }^{1} \mathbf{H}$ NMR (400 MHz, CDCl 3$): \delta 8.69$ (brs, 1H), 7.24-7.18 (m, 2H), 7.03 (t, $\left.J=7.5 \mathrm{~Hz}, 1 \mathrm{H}\right)$, $6.90(\mathrm{~d}, J=7.6 \mathrm{~Hz}, 1 \mathrm{H}), 5.76(\mathrm{ddt}, J=16.9,10.2,6.7 \mathrm{~Hz}, 1 \mathrm{H}), 5.07-4.89(\mathrm{~m}, 2 \mathrm{H}), 3.48(\mathrm{t}, J=$ $6.0 \mathrm{~Hz}, 1 \mathrm{H}), 2.08(\mathrm{q}, J=7.1 \mathrm{~Hz}, 2 \mathrm{H}), 1.98(\mathrm{ddd}, J=16.4,10.6,6.0 \mathrm{~Hz}, 2 \mathrm{H}), 1.57-1.37(\mathrm{~m}, 2 \mathrm{H})$. ${ }^{13} \mathbf{C}\left\{{ }^{1} \mathbf{H}\right\}$ NMR (100 MHz, CDCl $): \delta 180.6(\mathrm{C}=\mathrm{O}), 141.7(\mathrm{C}), 138.3(\mathrm{CH}), 129.9(\mathrm{C}), 128.0$ $(\mathrm{CH}), 124.3(\mathrm{CH}), 122.4(\mathrm{CH}), 115.0\left(\mathrm{CH}_{2}\right), 109.8(\mathrm{CH}), 46.1(\mathrm{CH}), 33.8\left(\mathrm{CH}_{2}\right), 30.1\left(\mathrm{CH}_{2}\right)$, $25.1\left(\mathrm{CH}_{2}\right)$. FT-IR (neat, $\mathbf{c m}^{-1}$ ): 3204, 2924, 1698, 1619, 1485, 1439, 1336, 1217, 1100. HRMS (ESI $\left.{ }^{+}\right): m / z$ calcd. for $\mathrm{C}_{13} \mathrm{H}_{15} \mathrm{NONa}[\mathrm{M}+\mathrm{Na}]^{+} 224.1046$, found 224.1043 .

\section{3-Pentylindolin-2-one $1 \mathrm{~h}$}

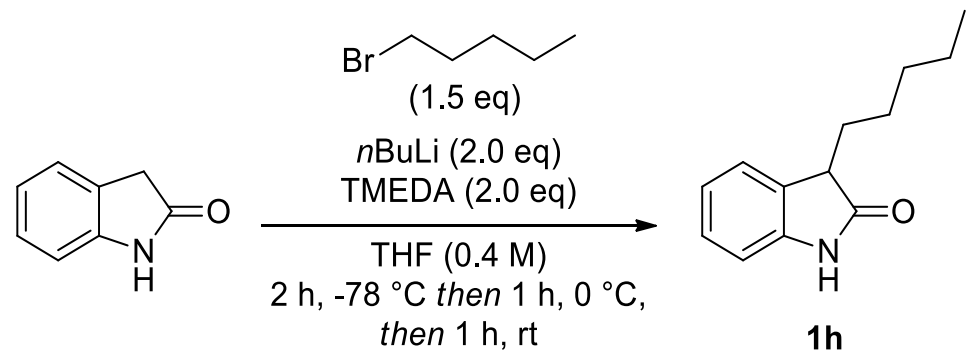

To a solution of oxindole $(2.0 \mathrm{~g}, 15.0 \mathrm{mmol}, 1.0$ equiv. $)$ in THF $(60 \mathrm{~mL})$ at $-78{ }^{\circ} \mathrm{C}$ was added $n \operatorname{BuLi}(2.5 \mathrm{M}$ in hexanes, $12.0 \mathrm{~mL}, 30.0 \mathrm{mmol}, 2.0$ equiv.) and TMEDA (4.51 mL, $30.0 \mathrm{mmol}$, 2.0 equiv.) and the resulting solution was stirred for $2 \mathrm{~h}$. 1-Bromopentane $(2.79 \mathrm{~mL}, 22.5 \mathrm{mmol}$, 1.1 equiv.) was then added dropwise and the solution was slowly warmed up to $0{ }^{\circ} \mathrm{C}$ and stirred for $1 \mathrm{~h}$ at that temperature and for $1 \mathrm{~h}$ at $\mathrm{rt}$. The reaction was then quenched with aq. $\mathrm{NH}_{4} \mathrm{Cl}$ and extracted with ethyl acetate. The combined organic layers were washed with brine, dried over $\mathrm{MgSO}_{4}$, and concentrated in vacuo. The crude was purified by $\mathrm{FC}\left(80 \mathrm{~g} \mathrm{SiO}_{2}\right.$, heptane/ethyl acetate, 100/0 to 70/30, $20 \mathrm{CV}$ ) to afford $\mathbf{1 h}$ as a light yellow oil $(1.18 \mathrm{~g}, 5.8 \mathrm{mmol}, 39 \%)$.

${ }^{1}$ H NMR (600 MHz, CDCl 3$): \delta 9.37$ (brs, 1H), $7.21(\mathrm{~m}, 2 \mathrm{H}), 7.02(\mathrm{t}, J=7.5 \mathrm{~Hz}, 1 \mathrm{H}), 6.93$ (d, $J=7.8 \mathrm{~Hz}, 1 \mathrm{H}), 3.48(\mathrm{t}, J=6.0 \mathrm{~Hz}, 1 \mathrm{H}), 2.02-1.90(\mathrm{~m}, 2 \mathrm{H}), 1.46-1.39(\mathrm{~m}, 1 \mathrm{H}), 1.36-1.31(\mathrm{~m}$, 
1H), 1.31-1.27 (m, 4H), $0.85(\mathrm{dd}, J=9.3,4.7 \mathrm{~Hz}, 3 \mathrm{H}) .{ }^{13} \mathbf{C}\left\{{ }^{1} \mathbf{H}\right\}$ NMR (150 MHz, CDCl $): \delta$ $181.4(\mathrm{C}=\mathrm{O}), 141.9(\mathrm{C}), 130.1(\mathrm{C}), 127.9(\mathrm{CH}), 124.2(\mathrm{CH}), 122.3(\mathrm{CH}), 110.0(\mathrm{CH}), 46.4$ $(\mathrm{CH}), 31.9\left(\mathrm{CH}_{2}\right), 30.6\left(\mathrm{CH}_{2}\right), 25.6\left(\mathrm{CH}_{2}\right), 22.5\left(\mathrm{CH}_{2}\right), 14.1\left(\mathrm{CH}_{3}\right)$. HRMS (ESI $\left.{ }^{+}\right): m / z$ calcd. for $\mathrm{C}_{13} \mathrm{H}_{17} \mathrm{NONa}[\mathrm{M}+\mathrm{Na}]^{+}$226.1202, found 226.1202. Spectral and physical data are in accordance with literature. ${ }^{2}$

\section{3-((1,3-Dioxolan-2-yl)methyl)indolin-2-one $1 \mathrm{i}$}

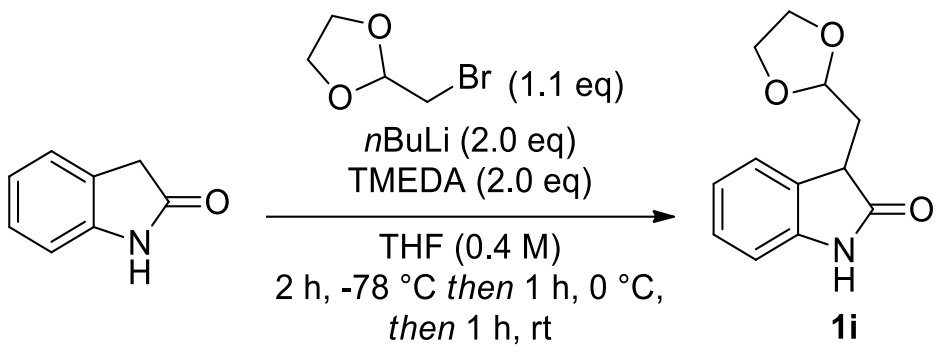

To a solution of oxindole $(2.0 \mathrm{~g}, 15.0 \mathrm{mmol}, 1.0$ equiv. $)$ in THF $(60 \mathrm{~mL})$ at $-78^{\circ} \mathrm{C}$ was added $n \operatorname{BuLi}(2.5 \mathrm{M}$ in hexanes, $12.0 \mathrm{~mL}, 30.0 \mathrm{mmol}, 2.0$ equiv.) and TMEDA (4.51 mL, $30.0 \mathrm{mmol}$, 2.0 equiv.) and the resulting solution was stirred for $2 \mathrm{~h}$. 2-Bromomethyl-1,3-dioxolane (1.71 $\mathrm{mL}, 16.5 \mathrm{mmol}, 1.1$ equiv.) was then added dropwise and the solution was slowly warmed up to $0^{\circ} \mathrm{C}$ and stirred for $1 \mathrm{~h}$ at that temperature and for $1 \mathrm{~h}$ at $\mathrm{rt}$. The reaction was then quenched with aq. $\mathrm{NH}_{4} \mathrm{Cl}$ and extracted with ethyl acetate. The combined organic layers were washed with brine, dried over $\mathrm{MgSO}_{4}$, and concentrated in vacuo. The crude was purified by FC ( $80 \mathrm{~g}$ $\mathrm{SiO}_{2}$, heptane/ethyl acetate: $100 / 0$ to $60 / 40,20 \mathrm{CV}$ ) to afford $1 \mathbf{i}$ as a white solid $(529 \mathrm{mg}$, $2.41 \mathrm{mmol}, 16 \%)$.

${ }^{1}$ H NMR (400 MHz, CDCl3): $\delta 7.87$ (brs, $\left.1 \mathrm{H}\right), 7.35$ (d, $\left.J=7.4 \mathrm{~Hz}, 1 \mathrm{H}\right), 7.21(\mathrm{t}, J=7.8 \mathrm{~Hz}$, $1 \mathrm{H}), 7.02(\mathrm{t}, J=7.2 \mathrm{~Hz}, 1 \mathrm{H}), 6.87(\mathrm{~d}, J=7.8 \mathrm{~Hz}, 1 \mathrm{H}), 5.20(\mathrm{dd}, J=5.9,3.8 \mathrm{~Hz}, 1 \mathrm{H}), 4.04-3.82$ $(\mathrm{m}, 4 \mathrm{H}), 3.64(\mathrm{t}, J=6.2 \mathrm{~Hz}, 1 \mathrm{H}), 2.41(\mathrm{ddd}, J=14.2,5.8,3.8 \mathrm{~Hz}, 1 \mathrm{H}), 2.17(\mathrm{dt}, J=14.2,6.2$ $\mathrm{Hz}, 1 \mathrm{H}) .{ }^{13} \mathbf{C}\left\{{ }^{1} \mathbf{H}\right\}$ NMR (100 MHz, CDCl $): \delta 179.7(\mathrm{C}=\mathrm{O}), 141.3(\mathrm{C}), 129.4(\mathrm{C}), 128.1(\mathrm{CH})$, $125.1(\mathrm{CH}), 122.4(\mathrm{CH}), 109.6(\mathrm{CH}), 102.3(\mathrm{CH}), 66.2\left(\mathrm{CH}_{2}\right), 66.0\left(\mathrm{CH}_{2}\right), 42.2(\mathrm{CH}), 34.4$ $\left(\mathrm{CH}_{2}\right)$. FT-IR (neat, $\left.\mathbf{c m}^{-1}\right)$ : 3174, 3130, 3078, 3035, 2953, 2888, 2849, 1694, 1619, 1470, 1140. HRMS (ESI $\left.{ }^{+}\right): m / z$ calcd. for $\mathrm{C}_{12} \mathrm{H}_{13} \mathrm{NO}_{3} \mathrm{Na}[\mathrm{M}+\mathrm{Na}]^{+} 242.0788$, found 242.0785.

\section{2-(2-Oxoindolin-3-yl)acetonitrile $1 \mathbf{j}$}

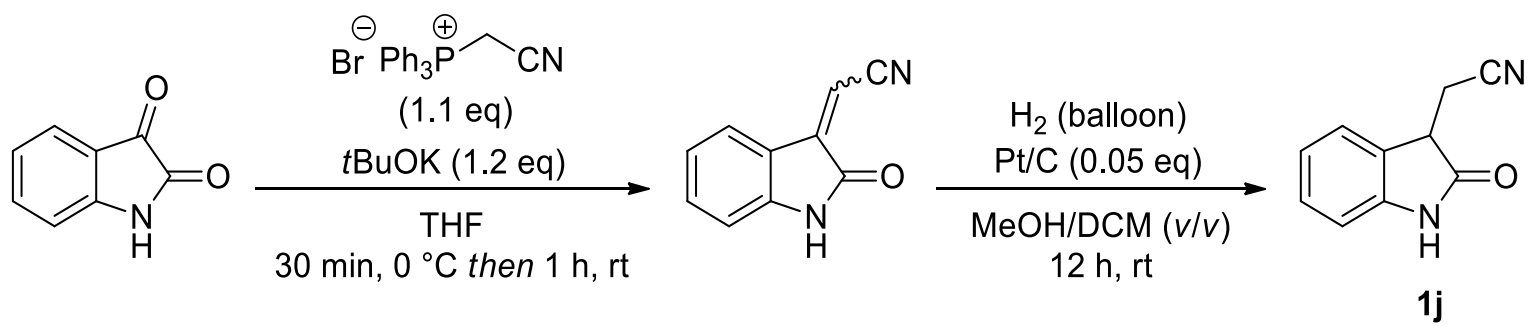

In a two-neck flask, under argon, (cyanomethyl)triphenylphosphonium chloride $(3.79 \mathrm{~g}$, $11.2 \mathrm{mmol}, 1.1$ equiv. $)$ was dissolved in THF $(10 \mathrm{~mL})$ and cooled in an ice-bath. $t \mathrm{BuOK}(1.42 \mathrm{~g}$, $12.2 \mathrm{mmol} ; 1.2$ equiv.) was added and the mixture was stirred at $0{ }^{\circ} \mathrm{C}$ for $30 \mathrm{~min}$. Then, isatin (1.5 g, $10.2 \mathrm{mmol}, 1.0$ equiv.) was added portion wise and stirred at $\mathrm{rt}$ for $1 \mathrm{~h}$. The reaction was quenched with $\mathrm{H}_{2} \mathrm{O}$, extracted with ethyl acetate, dried over $\mathrm{MgSO}_{4}$ and concentrated under 
reduced pressure. 2-(2-Oxoindolin-3-ylidene)acetonitrile (1.2 g, $7.08 \mathrm{mmol}, 69 \%)$ was obtained as an orange solid.

2-(2-Oxoindolin-3-ylidene)acetonitrile (1.2 g, $7.08 \mathrm{mmol}, 1.0$ equiv.) was dissolved in a mixture of $\mathrm{MeOH} / \mathrm{DCM}(24 \mathrm{~mL}, v / v)$. Pt/C $(69.0 \mathrm{mg}, 0.350 \mathrm{mmol}, 0.05$ equiv.) was added to this solution, and the resulting mixture was stirred under hydrogen atmosphere (balloon) for $12 \mathrm{~h}$ at $\mathrm{rt}$. The reaction mixture was then passed through celite, washed with DCM and concentrated in vacuo. The brown sticky solid was recrystallized in DCM and filtrated to afford $\mathbf{1 j}$ as a white solid (426 g, $2.47 \mathrm{mmol}, 35 \%)$.

${ }^{1} \mathbf{H}$ NMR (600 MHz, CDCl 3$): \delta 8.37$ (brs, $\left.1 \mathrm{H}\right), 7.49(\mathrm{~d}, J=7.5 \mathrm{~Hz}, 1 \mathrm{H}), 7.31(\mathrm{t}, J=7.7 \mathrm{~Hz}$, $1 \mathrm{H}), 7.11(\mathrm{t}, J=7.5 \mathrm{~Hz}, 1 \mathrm{H}), 6.94(\mathrm{~d}, J=7.7 \mathrm{~Hz}, 1 \mathrm{H}), 3.71(\mathrm{dd}, J=8.9,4.8 \mathrm{~Hz}, 1 \mathrm{H}), 3.10(\mathrm{dd}$, $J=16.9,4.8 \mathrm{~Hz}, 1 \mathrm{H}), 2.75(\mathrm{dd}, J=16.9,8.9 \mathrm{~Hz}, 1 \mathrm{H}) .{ }^{\mathbf{1 3}} \mathbf{C}\left\{{ }^{1} \mathbf{H}\right\}$ NMR (150 MHz, CDCl $\left.\mathbf{3}\right): \delta$ $176.4(\mathrm{C}=\mathrm{O}), 141.3(\mathrm{C}), 129.6(\mathrm{CH}), 126.3(\mathrm{C}), 124.7(\mathrm{CH}), 123.4(\mathrm{CH}), 117.2(\mathrm{C}), 110.4(\mathrm{CH})$, $41.9(\mathrm{CH}), 19.0\left(\mathrm{CH}_{2}\right)$. HRMS (ESI $\left.{ }^{+}\right): \mathrm{m} / z$ calcd. for $\mathrm{C}_{10} \mathrm{H}_{8} \mathrm{~N}_{2} \mathrm{ONa}[\mathrm{M}+\mathrm{Na}]^{+} 195.0529$, found 195.0530. Spectral and physical data are in accordance with literature. ${ }^{5}$

\section{Ethyl 2-(2-oxoindolin-3-yl)acetate 1k}<smiles>O=C1Nc2ccccc2C1=O</smiles>

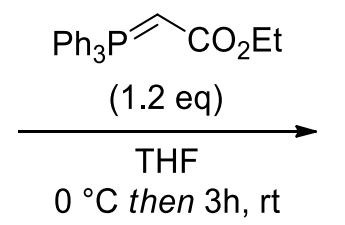<smiles>O=C=C1C(=O)Nc2ccccc21</smiles>

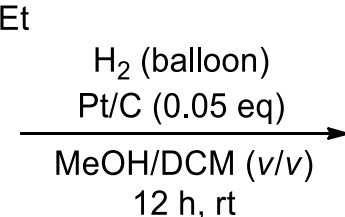<smiles>CCOC(=O)CC1C(=O)Nc2ccccc21</smiles>

$1 \mathrm{k}$

In a two-neck flask, under argon, ethyl(triphenylphosphoranylidene)acetate $(2.8 \mathrm{~g}, 8.20 \mathrm{mmol}$, 1.2 equiv.) was dissolved in THF $(10.0 \mathrm{~mL})$ and cooled in an ice-bath. Then, isatin $(1.0 \mathrm{~g}$, $6.80 \mathrm{mmol}, 1.0$ equiv.) was added portion wise and stirred at $\mathrm{rt}$ for $3 \mathrm{~h}$. The reaction was quenched with $\mathrm{H}_{2} \mathrm{O}$, extracted with ethyl acetate, dried over $\mathrm{MgSO}_{4}$ and concentrated under reduced pressure. The crude product was purified by $\mathrm{FC}\left(40 \mathrm{~g} \mathrm{SiO}_{2}\right.$, heptane/ethyl acetate, $100 / 0$ to $70 / 30,15 \mathrm{CV}$ ) to provide ethyl 2-(2-oxoindolin-3-ylidene)acetate (1.06 g, $4.88 \mathrm{mmol}$, $72 \%$ ) as an orange solid.

Ethyl 2-(2-oxoindolin-3-ylidene)acetate (1.0 g, $4.6 \mathrm{mmol}, 1.0$ equiv.) was dissolved in a mixture of $\mathrm{MeOH} / \mathrm{DCM}(20 \mathrm{~mL}, v / v)$. Pt/C (44.9 mg, $0.23 \mathrm{mmol}, 0.05$ equiv.) was added to this solution, and the resulting mixture was stirred under hydrogen atmosphere (balloon) for $12 \mathrm{~h}$ at $\mathrm{rt}$. The reaction mixture was then passed through celite, washed with DCM and concentrated in vacuo to afford $1 \mathbf{k}$ as a pale yellow solid (1.0 g, $4.6 \mathrm{mmol}, 99 \%)$.

${ }^{1} \mathbf{H}$ NMR (400 MHz, CDCl 3$): ~ \delta 8.89$ (brs, 1H), 7.29-7.21 (m, 2H), 7.02 (t, $\left.J=7.5 \mathrm{~Hz}, 1 \mathrm{H}\right)$, $6.92(\mathrm{~d}, J=7.7 \mathrm{~Hz}, 1 \mathrm{H}), 4.21-4.11(\mathrm{~m}, 2 \mathrm{H}), 3.86-3.79(\mathrm{~m}, 1 \mathrm{H}), 3.09(\mathrm{dd}, J=16.9,3.7 \mathrm{~Hz}, 1 \mathrm{H})$, $2.85(\mathrm{dd}, J=16.9,7.9 \mathrm{~Hz}, 1 \mathrm{H}), 1.22(\mathrm{td}, J=7.1,0.9 \mathrm{~Hz}, 3 \mathrm{H}) .{ }^{13} \mathbf{C}\left\{{ }^{1} \mathbf{H}\right\}$ NMR $(\mathbf{1 0 0} \mathbf{~ M H z}$, CDCl$\left._{3}\right): \delta 179.5(\mathrm{C}=\mathrm{O}), 171.2(\mathrm{C}=\mathrm{O}), 141.8(\mathrm{C}), 128.9(\mathrm{C}), 128.4(\mathrm{CH}), 124.2(\mathrm{CH}), 122.6$ $(\mathrm{CH}), 110.0(\mathrm{CH}), 61.1\left(\mathrm{CH}_{2}\right), 42.5(\mathrm{CH}), 34.9\left(\mathrm{CH}_{2}\right), 14.2\left(\mathrm{CH}_{3}\right)$. HRMS (ESI $\left.{ }^{+}\right): \mathrm{m} / z$ calcd. for $\mathrm{C}_{12} \mathrm{H}_{13} \mathrm{NO}_{3} \mathrm{Na}[\mathrm{M}+\mathrm{Na}]^{+} 242.0788$, found 242.0788. Spectral and physical data are in accordance with literature. ${ }^{6}$ 


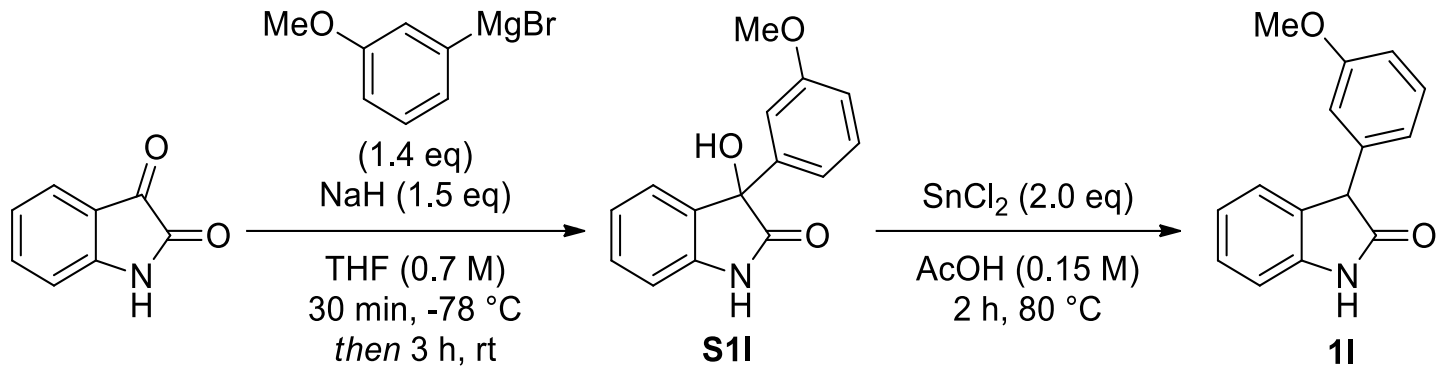

General procedure $\mathbf{B}$ was followed with isatin $(2.0 \mathrm{~g}, 13.6 \mathrm{mmol})$ and 3-methoxyphenylmagnesium bromide (1 $\mathrm{M}$ in 2-MeTHF, $19.0 \mathrm{~mL}, 19.0 \mathrm{mmol}$ ) to afford $\mathbf{S 1 l}$ as a white solid (2.75 g, 10.8 mmol, 79\%). ${ }^{1}$ H NMR (400 MHz, DMSO-d6): $\delta 10.39$ (s, 1H), 7.24 $(\mathrm{td}, J=7.7,1.1 \mathrm{~Hz}, 1 \mathrm{H}), 7.19(\mathrm{t}, J=8.0 \mathrm{~Hz}, 1 \mathrm{H}), 7.09(\mathrm{~d}, J=7.2 \mathrm{~Hz}, 1 \mathrm{H}), 6.98-6.93(\mathrm{~m}, 2 \mathrm{H})$, $6.89(\mathrm{~d}, J=7.7 \mathrm{~Hz}, 1 \mathrm{H}), 6.83(\mathrm{dd}, J=8.0,2.2 \mathrm{~Hz}, 1 \mathrm{H}), 6.69(\mathrm{~d}, J=7.8 \mathrm{~Hz}, 1 \mathrm{H}), 6.62(\mathrm{~s}, 1 \mathrm{H})$, 3.72 (s, 3H). ${ }^{13} \mathbf{C}\left\{{ }^{1} \mathbf{H}\right\}$ NMR (100 MHz, DMSO-d 6$): \delta 178.3(\mathrm{C}=\mathrm{O}), 159.1(\mathrm{C}), 143.1$ (C), 141.9 (C), $133.7(\mathrm{C}), 129.2(\mathrm{CH}), 129.2(\mathrm{CH}), 124.7(\mathrm{CH}), 122.0(\mathrm{CH}), 117.5(\mathrm{CH}), 112.5(\mathrm{CH})$, $111.5(\mathrm{CH}), 109.8(\mathrm{CH}), 77.2(\mathrm{C}), 55.0\left(\mathrm{CH}_{3}\right)$. HRMS (ESI $\left.{ }^{+}\right): \mathrm{m} / z$ calcd. for $\mathrm{C}_{15} \mathrm{H}_{13} \mathrm{NO}_{3} \mathrm{Na}$ $[\mathrm{M}+\mathrm{Na}]^{+} 278.0788$, found 278.0804. Spectral and physical data are in accordance with literature. ${ }^{9}$

The general procedure $\mathbf{C}$ was followed with $\mathbf{S 1 I}$ (2.0 g, $7.83 \mathrm{mmol})$. Purification was performed with flash column chromatography over silica gel (40 g SiO 2 , heptane/EtOAc, 100/0 to 70/30, gradient) to afford $\mathbf{1 l}$ as a white solid (1.84 g, $7.69 \mathrm{mmol}, 98 \%)$. ${ }^{\mathbf{1}} \mathbf{H}$ NMR (400 $\left.\mathbf{~ M H z , ~ C D C l} \mathbf{3}\right)$ : $\delta 8.69$ (brs, 1H), 7.25 (m, 2H), 7.13 (d, $J=7.5 \mathrm{~Hz}, 1 \mathrm{H}), 7.02$ (t, $J=7.5 \mathrm{~Hz}, 1 \mathrm{H}), 6.93$ (d, $J=$ $7.8 \mathrm{~Hz}, 1 \mathrm{H}), 6.84$ (dd, $J=12.8,5.0 \mathrm{~Hz}, 2 \mathrm{H}), 6.77-6.75(\mathrm{~m}, 1 \mathrm{H}), 4.61(\mathrm{~s}, 1 \mathrm{H}), 3.78(\mathrm{~s}, 3 \mathrm{H})$. ${ }^{13} \mathbf{C}\left\{{ }^{1} \mathbf{H}\right\}(\mathbf{1 0 0} \mathbf{M H z}, \mathbf{C D C l} 3): \delta 178.5(\mathrm{C}=\mathrm{O}), 160.1(\mathrm{C}), 141.7(\mathrm{C}), 138.0(\mathrm{C}), 130.1(\mathrm{CH}), 129.6$ (C), $128.6(\mathrm{CH}), 125.5(\mathrm{CH}), 122.9(\mathrm{CH}), 121.0(\mathrm{CH}), 114.5(\mathrm{CH}), 113.2(\mathrm{CH}), 110.1(\mathrm{CH})$, $55.4\left(\mathrm{CH}_{3}\right), 52.7(\mathrm{CH}) . \mathbf{H R M S}\left(\mathbf{E S I}^{+}\right): \mathrm{m} / z$ calcd. for $\mathrm{C}_{15} \mathrm{H}_{13} \mathrm{NO}_{2} \mathrm{Na}[\mathrm{M}+\mathrm{Na}]^{+} 262.0838$, found 262.0838. Spectral and physical data are in accordance with literature. ${ }^{9}$

\section{3-(4-Methoxyphenyl)indolin-2-one $1 \mathrm{~m}$}

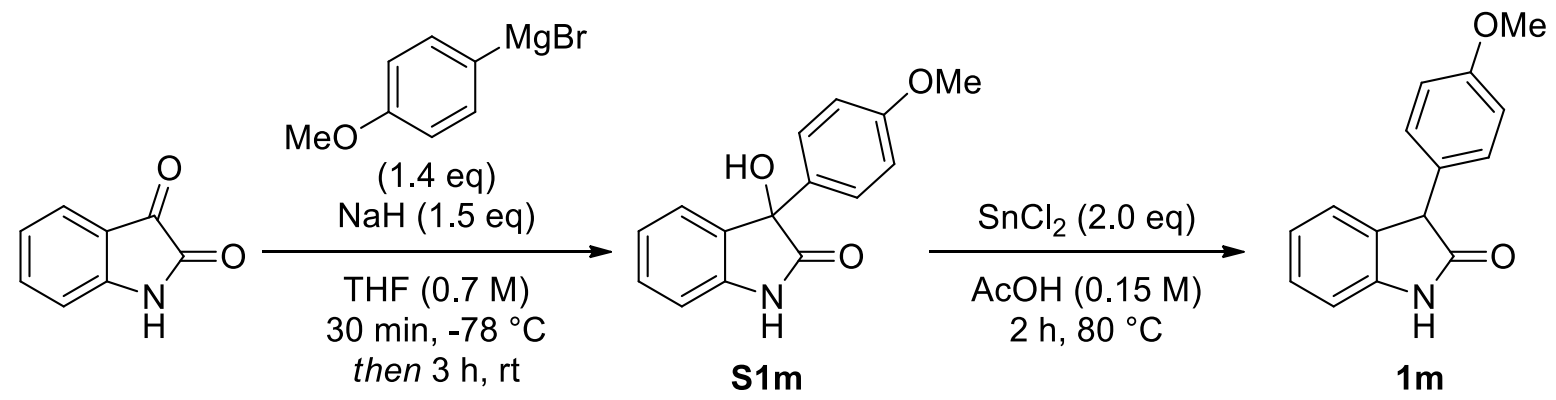

The general procedure $\mathbf{B}$ was followed with isatin $(3.0 \mathrm{~g}, 20.4 \mathrm{mmol})$ and 4-bromoanisole (3.57 mL, $28.5 \mathrm{mmol})$ to afford $\mathbf{S 1 m}$ as a white solid $(4.08 \mathrm{~g}, 16.0 \mathrm{mmol}, 78 \%) .{ }^{1} \mathbf{H}$ NMR (400 MHz, DMSO-d6): $\delta 10.32$ (s, 1H), 7.24 (td, $J=7.6,1.1 \mathrm{~Hz}, 1 \mathrm{H}), 7.18$ (d, $J=8.8 \mathrm{~Hz}, 2 \mathrm{H})$, $7.10(\mathrm{~d}, J=7.2 \mathrm{~Hz}, 1 \mathrm{H}), 6.96(\mathrm{t}, J=7.6 \mathrm{~Hz}, 1 \mathrm{H}), 6.89-6.85(\mathrm{~m}, 3 \mathrm{H}), 6.51(\mathrm{~s}, 1 \mathrm{H}), 3.71(\mathrm{~s}, 3 \mathrm{H})$. ${ }^{13} \mathbf{C}\left\{{ }^{1} \mathbf{H}\right\}$ NMR (100 MHz, DMSO-d6): $\delta 178.6(\mathrm{C}=\mathrm{O}), 158.7$ (C), 141.9 (C), 133.7 (C), 133.5 (C), $129.1(\mathrm{CH}), 126.8(2 \mathrm{CH}), 124.8(\mathrm{CH}), 121.9(\mathrm{CH}), 113.4(2 \mathrm{CH}), 109.8(\mathrm{CH}), 76.9(\mathrm{C})$, 
$55.1\left(\mathrm{CH}_{3}\right)$. FT-IR (neat, $\mathbf{c m}^{-1}$ ): 3270, 1718, 1684, 1610, 1509, 1466, 1347, 1297, 1250, 1177 , 1123, 1106, 1076, 1033. HRMS (ESI $\left.{ }^{+}\right): \mathrm{m} / z$ calcd. for $\mathrm{C}_{15} \mathrm{H}_{13} \mathrm{NO}_{3} \mathrm{Na}[\mathrm{M}+\mathrm{Na}]^{+} 278.0788$, found 278.0775. Spectral and physical data are in accordance with literature. ${ }^{8}$

The general procedure C was followed with S1m (3.5 g, $13.7 \mathrm{mmol})$ Purification was performed with flash column chromatography over silica gel $\left(40 \mathrm{~g} \mathrm{SiO}_{2}\right.$, heptane/EtOAc, 100/0 to 70/30, gradient) to afford $\mathbf{1 m}$ as a white solid (2.7 g, $11.7 \mathrm{mmol}, 82 \%)$. ${ }^{\mathbf{H}} \mathbf{H}$ NMR (400 MHz, CDCl 3$): \delta 8.61$ (brs, $1 \mathrm{H}), 7.24(\mathrm{dd}, J=11.1,3.4 \mathrm{~Hz}, 1 \mathrm{H}), 7.16-7.11(\mathrm{~m}, 3 \mathrm{H}), 7.03(\mathrm{td}, J=7.7$, $0.6 \mathrm{~Hz}, 1 \mathrm{H}), 6.93(\mathrm{~d}, J=7.7 \mathrm{~Hz}, 1 \mathrm{H}), 6.88(\mathrm{~d}, J=8.7 \mathrm{~Hz}, 2 \mathrm{H}), 4.59$ (s, 1H), 3.79 (s, 3H). ${ }^{13} \mathbf{C}\left\{{ }^{1} \mathbf{H}\right\}$ NMR (100 MHz, CDCl $)$ : $\delta 179.1(\mathrm{C}=\mathrm{O}), 159.3$ (C), $141.6(\mathrm{C}), 130.0$ (C), 129.7 $(2 \mathrm{CH}), 128.5(\mathrm{CH}+\mathrm{C}), 125.4(\mathrm{CH}), 122.9(\mathrm{CH}), 114.6(2 \mathrm{CH}), 110.1(\mathrm{CH}), 55.4\left(\mathrm{CH}_{3}\right), 52.0$ $(\mathrm{CH})$. HRMS (ESI $\left.{ }^{+}\right): \mathrm{m} / z$ calcd. for $\mathrm{C}_{15} \mathrm{H}_{13} \mathrm{NO}_{2} \mathrm{Na}[\mathrm{M}+\mathrm{Na}]^{+} 262.0838$, found 262.0838 . Spectral and physical data are in accordance with literature. ${ }^{7}$

\section{3-Phenylindolin-2-one 1n}

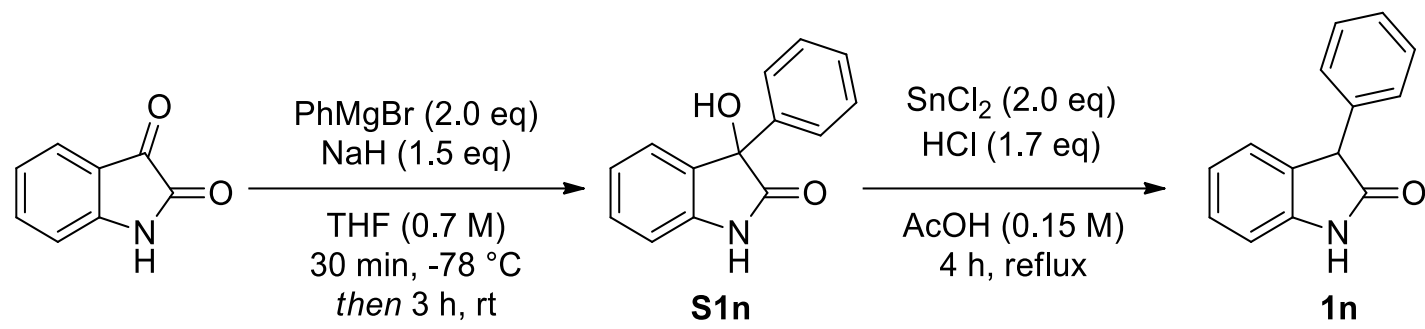

The general procedure $\mathbf{B}$ was followed with isatin $(2.0 \mathrm{~g}, 13.6 \mathrm{mmol})$ and phenylmagnesium bromide ( $3 \mathrm{M}_{\text {in }} \mathrm{Et}_{2} \mathrm{O}, 9.1 \mathrm{~mL}, 27.2 \mathrm{mmol}$ ) to afford $\mathrm{S1n}$ as a yellow solid $(1.2 \mathrm{~g}, 5.33 \mathrm{mmol}$, 39\%). ${ }^{1}$ H NMR (400 MHz, DMSO-d6): $\delta 10.40$ (s, 1H), 7.33-7.23 (m, 6H), 7.10 (d, $J=6.9$ Hz, $1 \mathrm{H}), 6.96(\mathrm{td}, J=7.7,1.1 \mathrm{~Hz}, 1 \mathrm{H}), 6.91(\mathrm{~d}, J=7.7 \mathrm{~Hz}, 1 \mathrm{H}), 6.63(\mathrm{~s}, 1 \mathrm{H}) .{ }^{13} \mathbf{C}\left\{{ }^{1} \mathbf{H}\right\} \mathbf{~ N M R}$ (100 MHz, DMSO-d6): $\delta 178.5$ (C=O), 142.0 (C), 141.6 (C), 133.8 (C), $129.2(\mathrm{CH}), 128.1$ $(2 \mathrm{CH}), 127.4(\mathrm{CH}), 125.4(2 \mathrm{CH}), 124.8(\mathrm{CH}), 122.1(\mathrm{CH}), 109.9(\mathrm{CH}), 77.3(\mathrm{C})$. FT-IR (neat,

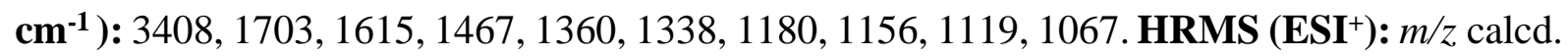
for $\mathrm{C}_{14} \mathrm{H}_{11} \mathrm{NO}_{2} \mathrm{Na}[\mathrm{M}+\mathrm{Na}]^{+}$248.0682, found 248.0670. Spectral and physical data are in accordance with literature. ${ }^{8}$

The general procedure C was followed with S1n (500 mg, $2.22 \mathrm{mmol}$ ) Purification was performed with flash column chromatography over silica gel $\left(40 \mathrm{~g} \mathrm{SiO}_{2}\right.$, heptane/EtOAc, 100/0 to 70/30, gradient) to afford $\mathbf{1 n}$ as a white solid (420 mg, $2.01 \mathrm{mmol}, 90 \%)$. ${ }^{\mathbf{1}} \mathbf{H}$ NMR (600 MHz, DMSO-d6): $\delta 10.51$ (brs, 1H), 7.36-7.31 (m, 2H), 7.29-7.25 (m, 1H), 7.24-7.20 (m, 1H), 7.16-7.12 (m, 2H), $7.03(\mathrm{~d}, J=7.4 \mathrm{~Hz}, 1 \mathrm{H}), 6.94(\mathrm{td}, J=7.5,1.0 \mathrm{~Hz}, 1 \mathrm{H}), 6.93-6.90(\mathrm{~m}, 1 \mathrm{H})$, 4.74 (s, 1H). ${ }^{13} \mathbf{C}\left\{{ }^{1} \mathbf{H}\right\}$ NMR (150 MHz, DMSO-d6): $\delta 177.2(\mathrm{C}=\mathrm{O}), 142.7$ (C), 137.7 (C), 130.0 (C), $128.7(2 \mathrm{CH}), 128.3(2 \mathrm{CH}), 128.1(\mathrm{CH}), 127.1(\mathrm{CH}), 124.8(\mathrm{CH}), 121.7(\mathrm{CH}), 109.5(\mathrm{CH})$, $51.8(\mathrm{CH})$. HRMS (ESI $\left.{ }^{+}\right): m / z$ calcd. for $\mathrm{C}_{14} \mathrm{H}_{11} \mathrm{NONa}[\mathrm{M}+\mathrm{Na}]^{+} 232.0733$, found 232.0732 . Spectral and physical data are in accordance with literature. ${ }^{9}$ 


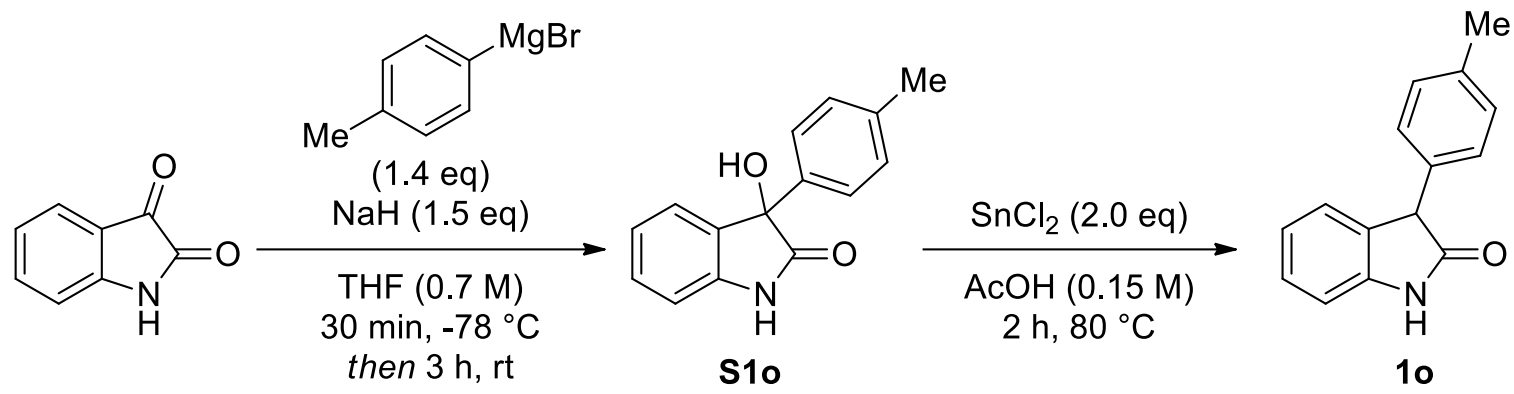

The general procedure $\mathbf{B}$ was followed with isatin $(1.0 \mathrm{~g}, 6.80 \mathrm{mmol})$ and 4-bromotoluene (1.17 mL, $9.52 \mathrm{mmol})$ to afford $\mathbf{S 1 o}$ as a yellow solid (690 mg, $2.88 \mathrm{mmol}, 42 \%) .{ }^{1} \mathbf{H}$ NMR (600 MHz, CDCl3): $\delta 8.20$ (brs, 1H), 7.29 (d, $J=8.1 \mathrm{~Hz}, 2 \mathrm{H}), 7.27-7.22$ (m, 2H), 7.13 (d, $J=8.1 \mathrm{~Hz}, 2 \mathrm{H}), 7.04(\mathrm{t}, J=7.6 \mathrm{~Hz}, 1 \mathrm{H}), 6.89(\mathrm{~d}, J=7.6 \mathrm{~Hz}, 1 \mathrm{H}), 2.31(\mathrm{~s}, 3 \mathrm{H}) .{ }^{13} \mathbf{C}\left\{{ }^{1} \mathbf{H}\right\} \mathbf{~ N M R}$ (150 MHz, CDCl3): $\delta 179.8(\mathrm{C}=\mathrm{O}), 140.6(\mathrm{C}), 138.4(\mathrm{C}), 137.0(\mathrm{C}), 132.3(\mathrm{C}), 130.0(\mathrm{CH})$, $129.5(2 \mathrm{CH}), 125.4(\mathrm{CH}), 125.4(2 \mathrm{CH}), 123.7(\mathrm{CH}), 110.6(\mathrm{CH}), 78.3(\mathrm{C}), 21.3\left(\mathrm{CH}_{3}\right)$. HRMS (ESI $\left.{ }^{+}\right): \mathrm{m} / z$ calcd. for $\mathrm{C}_{15} \mathrm{H}_{13} \mathrm{NO}_{2} \mathrm{Na}[\mathrm{M}+\mathrm{Na}]^{+} 262.0839$, found 262.0841. Spectral and physical data are in accordance with literature. ${ }^{8}$

The general procedure C was followed with S1o (600 mg, $2.51 \mathrm{mmol}$ ) Purification was performed with flash column chromatography over silica gel $\left(40 \mathrm{~g} \mathrm{SiO}_{2}\right.$, heptane/EtOAc, 100/0 to 70/30, gradient) to afford 10 as a white solid (553 mg, $2.48 \mathrm{mmol}, 99 \%) .{ }^{\mathbf{1}} \mathbf{H}$ NMR (400 MHz, CDCl3): $\delta 8.87$ (brs, $1 \mathrm{H}), 7.24(\mathrm{t}, J=7.8 \mathrm{~Hz}, 1 \mathrm{H}), 7.16(\mathrm{~d}, J=7.9 \mathrm{~Hz}, 2 \mathrm{H}), 7.13-7.10$ $(\mathrm{m}, 3 \mathrm{H}), 7.02(\mathrm{t}, J=7.5 \mathrm{~Hz}, 1 \mathrm{H}), 6.93(\mathrm{~d}, J=7.8 \mathrm{~Hz}, 1 \mathrm{H}), 4.60(\mathrm{~s}, 1 \mathrm{H}), 2.34(\mathrm{~s}, 3 \mathrm{H}) .{ }^{13} \mathbf{C}\left\{{ }^{1} \mathbf{H}\right\}$ NMR (100 MHz, CDCl3): $\delta 179.1(\mathrm{C}=\mathrm{O}), 141.8$ (C), 137.5 (C), 133.6 (C), 130.0 (C), 129.8 $(2 \mathrm{CH}), 128.5(2 \mathrm{CH}), 128.4(\mathrm{CH}), 125.4(\mathrm{CH}), 122.8(\mathrm{CH}), 110.1(\mathrm{CH}), 52.5(\mathrm{CH}), 21.3\left(\mathrm{CH}_{3}\right)$. HRMS (ESI $\left.{ }^{+}\right): m / z$ calcd. for $\mathrm{C}_{15} \mathrm{H}_{13} \mathrm{NONa}[\mathrm{M}+\mathrm{Na}]^{+} 246.0889$, found 246.0886. Spectral and physical data are in accordance with literature. ${ }^{9}$

\section{3-(4-(Tert-butyl)phenyl)indolin-2-one 1p}

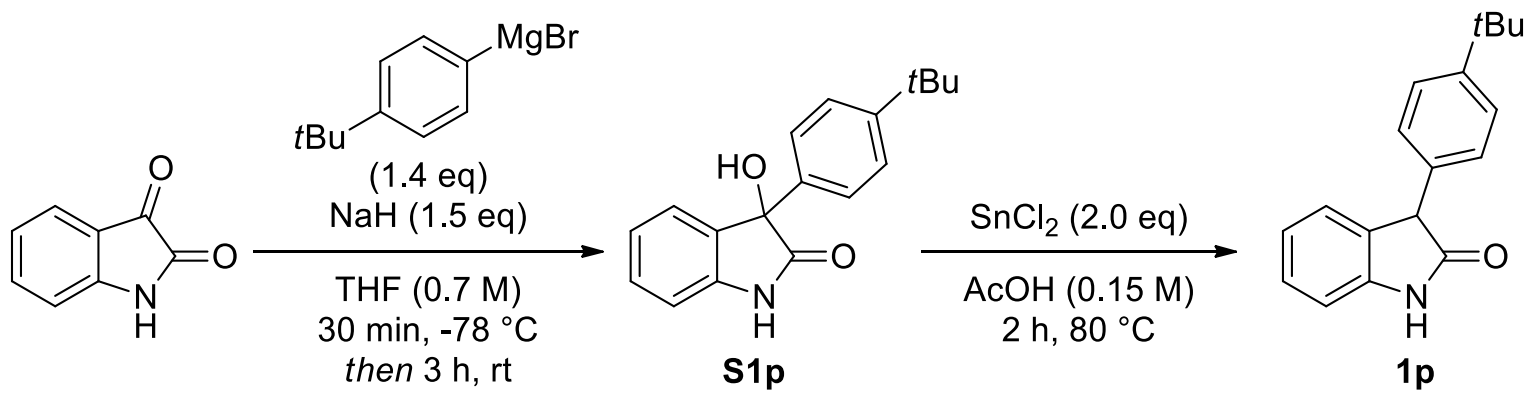

The general procedure $\mathbf{B}$ was followed with isatin $(1.0 \mathrm{~g}, 6.80 \mathrm{mmol})$ and 1-bromo-4-tertbutylbenzene (1.65 mL, $9.52 \mathrm{mmol})$ to afford $\mathbf{S 1 p}$ as a yellow solid $(1.11 \mathrm{~g}, 3.95 \mathrm{mmol}, 58 \%)$. ${ }^{1}$ H NMR (600 MHz, DMSO-d6): $\delta 10.36(\mathrm{~s}, 1 \mathrm{H}), 7.32(\mathrm{~d}, J=8.5 \mathrm{~Hz}, 2 \mathrm{H}), 7.24(\mathrm{td}, J=7.7$, $0.8 \mathrm{~Hz}, 1 \mathrm{H}), 7.19(\mathrm{~d}, J=8.5 \mathrm{~Hz}, 2 \mathrm{H}), 7.10(\mathrm{~d}, J=7.4 \mathrm{~Hz}, 1 \mathrm{H}), 6.96(\mathrm{t}, J=7.4 \mathrm{~Hz}, 1 \mathrm{H}), 6.88$ $(\mathrm{d}, J=7.7 \mathrm{~Hz}, 1 \mathrm{H}), 6.53(\mathrm{~s}, 1 \mathrm{H}), 1.24(\mathrm{~s}, 9 \mathrm{H}) .{ }^{13} \mathbf{C}\left\{{ }^{1} \mathbf{H}\right\}$ NMR (150 MHz, DMSO-d6): $\delta 178.6$ $(\mathrm{C}=\mathrm{O}), 149.8(\mathrm{C}), 141.9(\mathrm{C}), 138.6(\mathrm{C}), 133.8(\mathrm{C}), 129.2(\mathrm{CH}), 125.2(2 \mathrm{CH}), 124.9(2 \mathrm{CH})$, $124.8(\mathrm{CH}), 122.0(\mathrm{CH}), 109.8(\mathrm{CH}), 77.2(\mathrm{C}), 34.2(\mathrm{C}), 31.1\left(3 \mathrm{CH}_{3}\right)$. HRMS (ESI $\left.{ }^{+}\right)$: 
$m / z$ calcd. for $\mathrm{C}_{18} \mathrm{H}_{19} \mathrm{NO}_{2} \mathrm{Na}[\mathrm{M}+\mathrm{Na}]^{+}$304.1308, found 304.1306. Spectral and physical data are in accordance with literature. ${ }^{10}$

The general procedure C was followed with S1p (800 mg, $2.84 \mathrm{mmol}$ ) Purification was performed with flash column chromatography over silica gel (40 g SiO 2 , heptane/EtOAc, 100/0 to $70 / 30$, gradient) to afford $\mathbf{2 p}$ as a white solid (720 mg, $2.71 \mathrm{mmol}, 91 \%)$. ${ }^{\mathbf{1}} \mathbf{H}$ NMR (400 MHz, CDCl 3$): \delta 8.36$ (brs, $1 \mathrm{H}), 7.36(\mathrm{~d}, J=8.3 \mathrm{~Hz}, 2 \mathrm{H}), 7.27-7.22(\mathrm{~m}, 1 \mathrm{H}), 7.17-7.13(\mathrm{~m}, 3 \mathrm{H})$, $7.03(\mathrm{t}, J=7.5 \mathrm{~Hz}, 1 \mathrm{H}), 6.94(\mathrm{~d}, J=7.8 \mathrm{~Hz}, 1 \mathrm{H}), 4.62(\mathrm{~s}, 1 \mathrm{H}), 1.30(\mathrm{~s}, 9 \mathrm{H}) .{ }^{13} \mathbf{C}\left\{{ }^{1} \mathbf{H}\right\} \mathbf{~ N M R}$ (100 MHz, CDCl3): $\delta 178.7(\mathrm{C}=\mathrm{O}), 150.6(\mathrm{C}), 141.6(\mathrm{C}), 133.4(\mathrm{C}), 129.9(\mathrm{C}), 128.5(\mathrm{CH})$, $128.2(2 \mathrm{CH}), 126.1(2 \mathrm{CH}), 125.1(\mathrm{CH}), 122.9(\mathrm{CH}), 110.0(\mathrm{CH}), 52.3(\mathrm{CH}), 34.7(\mathrm{C}), 31.5$ $\left(3 \mathrm{CH}_{3}\right)$. HRMS (ESI $\left.{ }^{+}\right): \mathrm{m} / z$ calcd. for $\mathrm{C}_{18} \mathrm{H}_{19} \mathrm{NONa}[\mathrm{M}+\mathrm{Na}]^{+} 288.1359$, found 288.1356 . Spectral and physical data are in accordance with literature. ${ }^{7}$

\section{3-([1,1'-Biphenyl]-4-yl)indolin-2-one 1q}

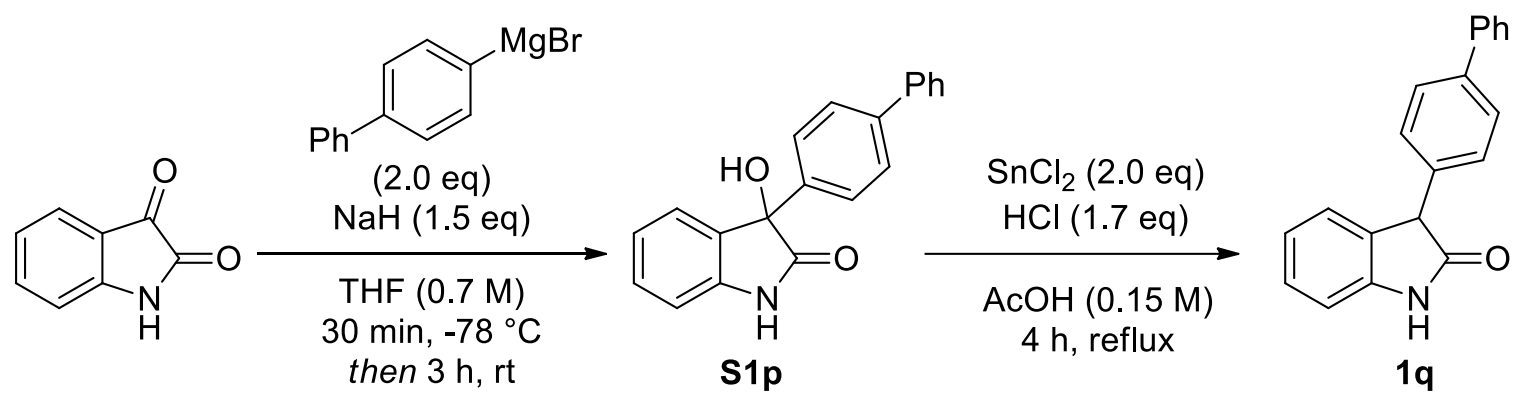

General procedure B was followed with isatin $(1.0 \mathrm{~g}, 6.80 \mathrm{mmol})$ and 4-bromobiphenyl (3.4 mL, $13.6 \mathrm{mmol})$ to afford S1q as a white solid (1.07 g, $3.54 \mathrm{mmol}, 52 \%)$. ${ }^{\mathbf{1}} \mathbf{H}$ NMR (600 MHz, DMSO-d6): $\delta 10.45$ (s, 1H), 7.69-7.59 (m, 4H), 7.52-7.42 (m, 2H), 7.42-7.33 (m, 3H), 7.33-7.24 (m, 1H), $7.16(\mathrm{~d}, J=6.7 \mathrm{~Hz}, 1 \mathrm{H}), 7.05-6.89(\mathrm{~m}, 2 \mathrm{H}), 6.70(\mathrm{~s}, 1 \mathrm{H}) .{ }^{13} \mathbf{C}\left\{{ }^{1} \mathbf{H}\right\} \mathbf{~ N M R}$ (150 MHz, DMSO-d6): $\delta 178.4$ (C=O), 142.0 (C), 140.7 (C), 139.9 (C), 139.4 (C), 133.6 (C), $129.3(\mathrm{CH}), 128.9(\mathrm{CH}), 127.5(\mathrm{CH}), 126.7(\mathrm{CH}), 126.5(\mathrm{CH}), 126.1(\mathrm{CH}), 124.8(\mathrm{CH})$, $122.1(\mathrm{CH}), 109.9(\mathrm{CH}), 77.2(\mathrm{C}) . \quad$ HRMS (ESI $\left.{ }^{+}\right): \mathrm{m} / z$ calcd. for $\mathrm{C}_{20} \mathrm{H}_{15} \mathrm{NO}_{2} \mathrm{Na} \quad[\mathrm{M}$ $+\mathrm{Na}]^{+}$324.0995, found 324.0991. Spectral and physical data are in accordance with literature. ${ }^{10}$

The general procedure C was followed with S1q (400 mg, $1.33 \mathrm{mmol}$ ) Purification was performed with flash column chromatography over silica gel (40 g SiO 2 , heptane/EtOAc, 100/0 to 70/30, gradient) to afford $\mathbf{1 q}$ as a white solid (337 mg, $1.18 \mathrm{mmol}, 89 \%) .{ }^{\mathbf{1}} \mathbf{H}$ NMR (600 MHz, DMSO-d6): $\delta 10.55$ (s, 1H), 7.68-7.59 (m, 4H), 7.48-7.42 (m, 2H), 7.37-7.34 (m, $1 \mathrm{H}), 7.23(\mathrm{dd}, J=11.9,5.4 \mathrm{~Hz}, 3 \mathrm{H}), 7.08(\mathrm{~d}, J=7.4 \mathrm{~Hz}, 1 \mathrm{H}), 6.97(\mathrm{td}, J=7.5,0.9 \mathrm{~Hz}, 1 \mathrm{H})$, $6.93(\mathrm{~d}, J=7.8 \mathrm{~Hz}, 1 \mathrm{H}), 4.81(\mathrm{~s}, 1 \mathrm{H}) .{ }^{13} \mathbf{C}\left\{{ }^{\mathbf{1}} \mathbf{H}\right\}$ NMR (150 MHz, DMSO-d 6$):$ $142.8(\mathrm{C}), 139.9(\mathrm{C}), 139.1(\mathrm{C}), 136.9(\mathrm{C}), 130.0(\mathrm{C}), 129.0(2 \mathrm{CH}), 129.0(2 \mathrm{CH}), 128.2(\mathrm{CH})$, $127.5(\mathrm{CH}), 127.1(2 \mathrm{CH}), 126.7(2 \mathrm{CH}), 124.9(\mathrm{CH}), 121.8(\mathrm{CH}), 109.6(\mathrm{CH}), 51.5(\mathrm{CH})$. FTIR (neat, $\mathbf{~ c m}^{-1}$ ): 3080, 2918, 1700, 1612, 1466, 1402, 1297, 1264, 1220, 1070. HRMS (ESI $\left.{ }^{+}\right): \mathrm{m} / \mathrm{z}$ calcd. for $\mathrm{C}_{20} \mathrm{H}_{15} \mathrm{NONa}[\mathrm{M}+\mathrm{Na}]^{+} 308.1046$, found 308.1044 . 


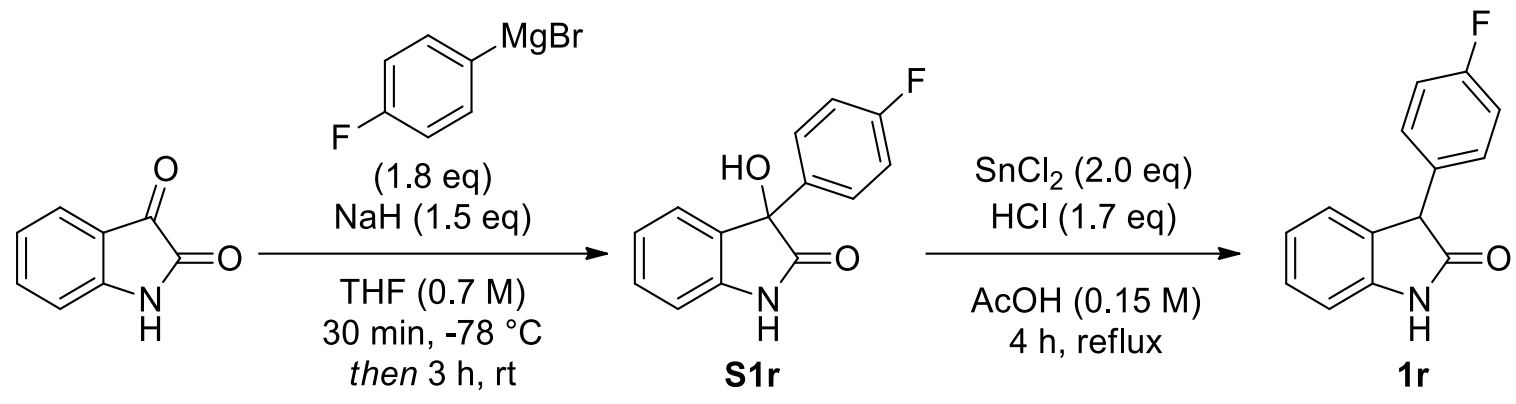

General procedure $\mathbf{B}$ was followed with isatin $(500 \mathrm{mg}, 3.40 \mathrm{mmol})$ and 4-fluorophenylmagnesium bromide (1.0 M in THF, $6.12 \mathrm{~mL}, 6.12 \mathrm{mmol})$ to afford $\mathbf{S 1 r}$ as a light yellow solid (452 mg, $1.86 \mathrm{mmol}, 55 \%) .{ }^{\mathbf{1}} \mathbf{H}$ NMR (600 MHz, DMSO-d6): $\delta 10.42$ (s, $1 \mathrm{H}), 7.32-7.27(\mathrm{~m}, 2 \mathrm{H}), 7.26(\mathrm{td}, J=7.7,1.2 \mathrm{~Hz}, 1 \mathrm{H}), 7.17-7.09(\mathrm{~m}, 3 \mathrm{H}), 6.98(\mathrm{td}, J=7.5,0.7$ $\mathrm{Hz}, 1 \mathrm{H}), 6.90$ (d, $J=7.7 \mathrm{~Hz}, 1 \mathrm{H}), 6.69$ (s, 1H). ${ }^{13} \mathbf{C}\left\{{ }^{1} \mathbf{H}\right\}$ NMR (150 MHz, DMSO-d6): $\delta 178.3$ $(\mathrm{C}=\mathrm{O}), 161.6\left(\mathrm{~d}, J^{\mathrm{CF}}=243.5 \mathrm{~Hz}, \mathrm{C}\right), 141.9(\mathrm{C}), 137.7\left(\mathrm{~d}, J^{\mathrm{CF}}=2.8 \mathrm{~Hz}, \mathrm{C}\right), 133.4(\mathrm{C}), 129.4$ $(\mathrm{CH}), 127.6\left(\mathrm{~d}, J^{\mathrm{CF}}=8.3 \mathrm{~Hz}, 2 \mathrm{CH}\right), 124.8(\mathrm{CH}), 122.2(\mathrm{CH}), 114.9\left(\mathrm{~d}, J^{\mathrm{CF}}=21.4 \mathrm{~Hz}, 2 \mathrm{CH}\right)$, $110.0(\mathrm{CH}), 76.8(\mathrm{C}) .{ }^{19}$ F NMR (565 MHz, DMSO-d6): $\delta$-115.23. FT-IR (neat, $\mathbf{~ c m}^{\mathbf{- 1}}$ ): 3433 , 3178 1693, 1620, 1602, 1503, 1472, 1229, 1199, 1172, 1121. HRMS (ESI $\left.{ }^{+}\right): \mathrm{m} / z$ calcd. for $\mathrm{C}_{14} \mathrm{H}_{10} \mathrm{NO}_{2} \mathrm{FNa}[\mathrm{M}+\mathrm{Na}]^{+}$266.0588, found 266.0591.

The general procedure C was followed with S1r (300 mg, $1.23 \mathrm{mmol}$ ) Purification was performed with flash column chromatography over silica gel (40 g SiO 2 , heptane/EtOAc, 100/0 to 70/30, gradient) to afford $1 \mathbf{r}$ as a white solid (150 mg, $0.66 \mathrm{mmol}, 54 \%)$. ${ }^{1} \mathbf{H}$ NMR (700 MHz, DMSO-d6): $\delta 10.53$ (brs, 1H), $7.23(\mathrm{t}, J=7.7 \mathrm{~Hz}, 1 \mathrm{H}), 7.20-7.15(\mathrm{~m}, 4 \mathrm{H}), 7.04$ (t, $J=$ $9.3 \mathrm{~Hz}, 1 \mathrm{H}), 6.96(\mathrm{td}, J=7.5,0.7 \mathrm{~Hz}, 1 \mathrm{H}), 6.91(\mathrm{~d}, J=7.8 \mathrm{~Hz}, 1 \mathrm{H}), 4.78(\mathrm{~s}, 1 \mathrm{H}) .{ }^{13} \mathbf{C}\left\{{ }^{1} \mathbf{H}\right\} \mathbf{~ N M R}$ (176 MHz, DMSO-d6): $\delta 177.1(\mathrm{C}=\mathrm{O}), 161.4\left(\mathrm{~d}, J^{\mathrm{CF}}=243.0 \mathrm{~Hz}, \mathrm{C}\right), 142.7(\mathrm{C}), 133.8\left(\mathrm{~d}, J^{\mathrm{CF}}\right.$ $=3.1 \mathrm{~Hz}, \mathrm{C}), 130.3\left(\mathrm{~d}, J^{\mathrm{CF}}=8.1 \mathrm{~Hz}, 2 \mathrm{CH}\right), 129.8(\mathrm{C}), 128.2(\mathrm{CH}), 124.8(\mathrm{CH}), 121.8(\mathrm{CH})$, $115.5\left(\mathrm{~d}, J^{\mathrm{CF}}=21.4 \mathrm{~Hz}, 2 \mathrm{CH}\right), 109.6(\mathrm{CH}), 50.9(\mathrm{CH}) .{ }^{19}$ F NMR (659 MHz, DMSO-d6): $\delta$ 155.63. HRMS $\left(\mathbf{E S I}^{+}\right): \mathrm{m} / z$ calcd. for $\mathrm{C}_{14} \mathrm{H}_{10} \mathrm{FNONa}[\mathrm{M}+\mathrm{Na}]^{+} 250.0639$, found 250.0638 . Spectral and physical data are in accordance with literature. ${ }^{9}$

\section{3-(4-Chlorophenyl)indolin-2-one 1s}

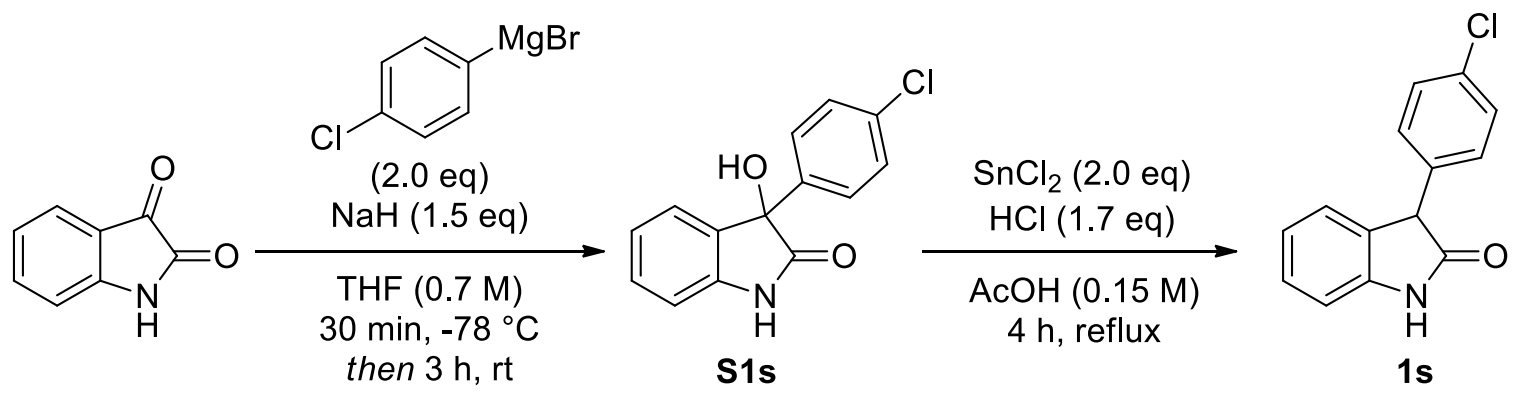

The general procedure B was followed with isatin $(500 \mathrm{mg}, 3.40 \mathrm{mmol})$ and 4-chlorophenylmagnesium bromide (1.0 M in THF, $6.8 \mathrm{~mL}, 6.80 \mathrm{mmol}$ ) to afford $\mathbf{S 1 s}$ as a light yellow solid (200 mg, $0.77 \mathrm{mmol}, 23 \%)$. ${ }^{\mathbf{1}}$ H NMR (600 MHz, DMSO-d6): $\delta 10.45$ (s, 1H), $7.32(\mathrm{~d}, J=8.5 \mathrm{~Hz}, 2 \mathrm{H}), 7.28-7.24(\mathrm{~m}, 3 \mathrm{H}), 7.10(\mathrm{~d}, J=7.3 \mathrm{~Hz}, 1 \mathrm{H}), 6.98(\mathrm{t}, J=7.5 \mathrm{~Hz}, 1 \mathrm{H})$, $6.91(\mathrm{~d}, J=7.8 \mathrm{~Hz}, 1 \mathrm{H}), 6.74(\mathrm{~s}, 1 \mathrm{H}) .{ }^{13} \mathbf{C}\left\{{ }^{1} \mathbf{H}\right\}$ NMR (150 MHz, DMSO-d6): $\delta 178.1(\mathrm{C}=\mathrm{O})$, 
$142.0(\mathrm{C}), 140.5(\mathrm{C}), 133.2(\mathrm{C}), 132.2(\mathrm{C}), 129.5(\mathrm{CH}), 128.2(2 \mathrm{CH}), 127.4(2 \mathrm{CH}), 124.8(\mathrm{CH})$, $122.2(\mathrm{CH}), 110.0(\mathrm{CH}), 76.9(\mathrm{C})$. FT-IR (neat, $\mathbf{c m}^{-1}$ ): 3189, 1714, 1620, 1489, 1471, 1396, 1181, 1102. HRMS (ESI $\left.{ }^{+}\right): \mathrm{m} / z$ calcd. for $\mathrm{C}_{14} \mathrm{H}_{10} \mathrm{NO}_{2} \mathrm{ClNa}[\mathrm{M}+\mathrm{Na}]^{+}$282.0298, found 282.0293.

The general procedure C was followed with S1s $(250 \mathrm{mg}, 0.96 \mathrm{mmol})$ Purification was performed with flash column chromatography over silica gel (40 g SiO 2 , heptane/EtOAc, 100/0 to $70 / 30$, gradient) to afford $1 \mathrm{~s}$ as a yellow solid (163 $\mathrm{mg}, 0.67 \mathrm{mmol}, 70 \%) .{ }^{1} \mathbf{H}$ NMR (600 MHz, CDCl $): \delta 8.39$ (brs, $1 \mathrm{H}), 7.32(\mathrm{~d}, J=8.5 \mathrm{~Hz}, 2 \mathrm{H}), 7.28-7.25(\mathrm{~m}, 1 \mathrm{H}), 7.17(\mathrm{~d}, J=8.5$ $\mathrm{Hz}, 2 \mathrm{H}), 7.11(\mathrm{~d}, J=7.4 \mathrm{~Hz}, 1 \mathrm{H}), 7.05(\mathrm{td}, J=7.5,0.6 \mathrm{~Hz}, 1 \mathrm{H}), 6.94(\mathrm{~d}, J=7.8 \mathrm{~Hz}, 1 \mathrm{H}), 4.61$ (s, 1H). ${ }^{13} \mathbf{C}\left\{{ }^{1} \mathbf{H}\right\}$ NMR (150 MHz, CDCl $)$ : $\delta 177.9(\mathrm{C}=\mathrm{O}), 141.6(\mathrm{C}), 136.0(\mathrm{C}), 133.8(\mathrm{C})$, $130.0(2 \mathrm{CH}), 129.2(2 \mathrm{CH}), 129.1(\mathrm{C}), 128.8(\mathrm{CH}), 125.5(\mathrm{CH}), 123.1(\mathrm{CH}), 110.1(\mathrm{CH}), 52.0$ (CH). FT-IR (neat, $\mathbf{c m}^{-1}$ ): 3175, $1702,1669,1617,1488,1470,1328,1218,1091$. HRMS $\left(\right.$ ESI $\left.^{+}\right): m / z$ calcd. for $\mathrm{C}_{14} \mathrm{H}_{10} \mathrm{NOClNa}[\mathrm{M}+\mathrm{Na}]^{+} 266.0343$, found 266.0340 .

\section{7-Chloro-3-(4-methoxyphenyl)indolin-2-one 3a}

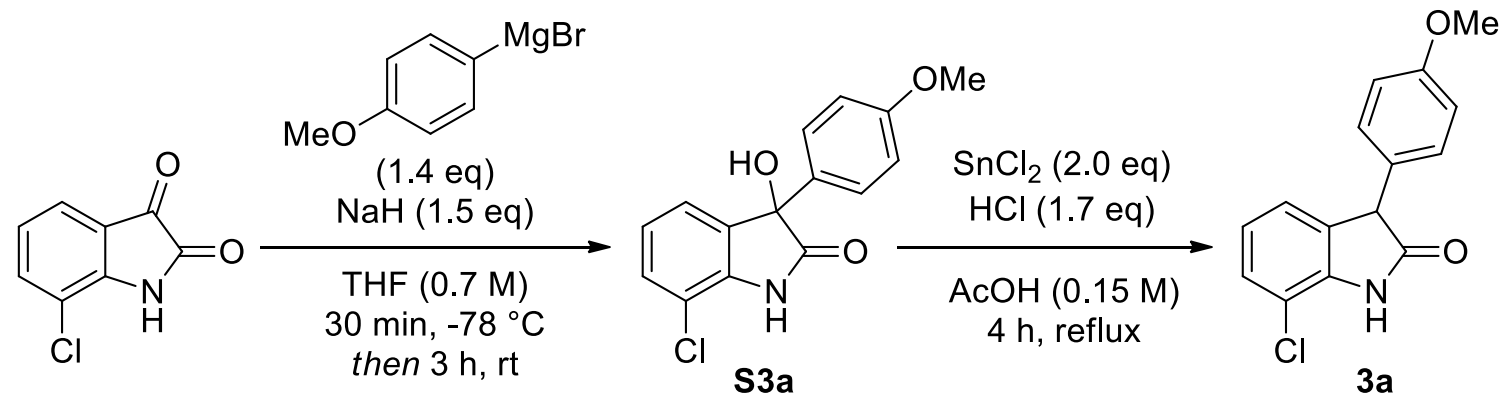

General procedure $\mathbf{B}$ was followed with 7-chloroisatin $(1.0 \mathrm{~g}, 5.51 \mathrm{mmol})$ and bromoanisole (1.38 mL, $11.0 \mathrm{mmol}, 2.0$ equiv.) to afford $\mathbf{S 3 a}$ as a yellow solid (1.06 g, $3.67 \mathrm{mmol}, 66 \%) .{ }^{1} \mathbf{H}$ NMR (600 MHz, CDCl3): $\delta 7.96(\mathrm{~s}, 1 \mathrm{H}), 7.27(\mathrm{~d}, J=8.9 \mathrm{~Hz}, 2 \mathrm{H}), 7.30-7.28(\mathrm{~m}, 1 \mathrm{H}), 7.11(\mathrm{~d}$, $J=7.4 \mathrm{~Hz}, 1 \mathrm{H}), 6.95(\mathrm{t}, J=7.9 \mathrm{~Hz}, 1 \mathrm{H}), 6.79(\mathrm{~d}, J=8.9 \mathrm{~Hz}, 2 \mathrm{H}), 3.71(\mathrm{~s}, 3 \mathrm{H}) .{ }^{13} \mathbf{C}\left\{{ }^{1} \mathbf{H}\right\} \mathbf{~ N M R}$ (151 MHz, CDCI3) $\delta 178.7$ (C=O), 160.0 (C), 138.3 (C), 133.5 (C), 131.4 (C), 129.8 (CH), $126.9(2 \mathrm{CH}), 124.5(\mathrm{CH}), 123.7(\mathrm{CH}), 115.7(\mathrm{C}), 114.3(2 \mathrm{CH}), 78.9(\mathrm{C}), 55.5\left(\mathrm{CH}_{3}\right)$. FT-IR (neat, cm$^{-1}$ ): 33013136172016171508147214541249118411661133 . HRMS (ESI'): $m / z$ calcd. for $\mathrm{C}_{15} \mathrm{H}_{12} \mathrm{ClNO}_{3} \mathrm{Na}[\mathrm{M}+\mathrm{Na}]^{+} 312.0398$, found 312.0396 .

General procedure $\mathbf{C}$ was followed with S3a (250 mg, $0.860 \mathrm{mmol}$ ) Purification was performed with flash column chromatography over silica gel (40 $\mathrm{g} \mathrm{SiO}_{2}$, heptane/EtOAc, 100/0 to 70/30, gradient) to afford 3a as a light yellow solid (140 mg, $0.510 \mathrm{mmol}, 59 \%)$. ${ }^{\mathbf{1}} \mathbf{H}$ NMR (500 MHz, DMSO-d6): $\delta 10.90(\mathrm{~s}, 1 \mathrm{H}), 7.28(\mathrm{dd}, J=6.7,2.1 \mathrm{~Hz}, 1 \mathrm{H}), 7.06(\mathrm{~d}, J=8.7 \mathrm{~Hz}, 2 \mathrm{H}), 6.97$ (d, $J$ $=6.7 \mathrm{~Hz}, 2 \mathrm{H}), 6.90(\mathrm{~d}, J=8.7 \mathrm{~Hz}, 2 \mathrm{H}), 4.82(\mathrm{~s}, 1 \mathrm{H}), 3.73(\mathrm{~s}, 3 \mathrm{H}) .{ }^{13} \mathbf{C}\left\{{ }^{1} \mathbf{H}\right\}$ NMR $(\mathbf{1 2 6} \mathbf{M H z}$, DMSO-d6): $\delta 177.3$ (C=O), 158.5 (C), 140.4 (C), 132.2 (C), 129.4 (2CH), 128.9 (C), 128.0 $(\mathrm{CH}), 123.4(\mathrm{CH}), 122.9(\mathrm{CH}), 114.2(2 \mathrm{CH}), 113.7(\mathrm{C}), 55.1\left(\mathrm{CH}_{3}\right), 51.7(\mathrm{CH})$. FT-IR (neat, $\mathbf{c m}^{-\mathbf{1}}$ ): $3134,3065,1706,1609,1512,1473,1453,1253,1175$. HRMS (ESI $\left.{ }^{+}\right): ~ m / z$ calcd. for $\mathrm{C}_{15} \mathrm{H}_{12} \mathrm{NO}_{2} \mathrm{ClNa}[\mathrm{M}+\mathrm{Na}]^{+}$296.0449, found 296.0448. 


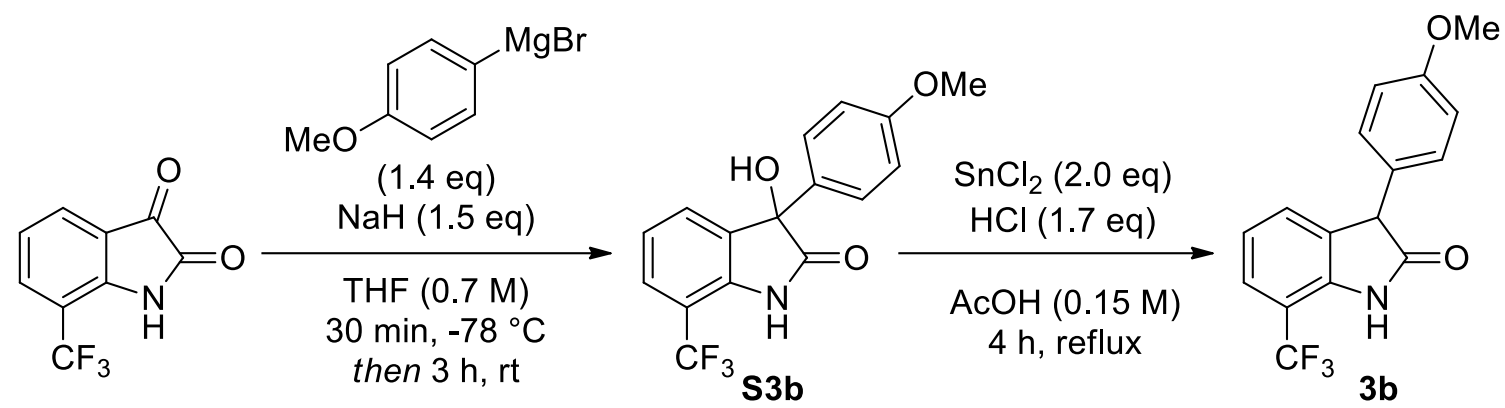

General procedure B was followed with 7-(trifluoromethyl)isatin (300 $\mathrm{mg}, 1.39 \mathrm{mmol})$ and bromoanisole ( $0.23 \mathrm{~mL}, 1.81 \mathrm{mmol}, 1.3$ equiv.) to afford $\mathbf{S 3 b}$ as a yellow solid (456 mg, $1.91 \mathrm{mmol}, 61 \%) .{ }^{1} \mathbf{H}$ NMR (500 MHz, CDCl 3$): \delta 8.13(\mathrm{~s}, 1 \mathrm{H}), 7.48(\mathrm{~d}, J=8.0 \mathrm{~Hz}, 1 \mathrm{H}), 7.44$ $(\mathrm{d}, J=7.4 \mathrm{~Hz}, 1 \mathrm{H}), 7.32(\mathrm{~d}, J=8.9 \mathrm{~Hz}, 2 \mathrm{H}), 7.15(\mathrm{t}, J=7.8 \mathrm{~Hz}, 1 \mathrm{H}), 6.86(\mathrm{~d}, J=8.9 \mathrm{~Hz}, 2 \mathrm{H})$,

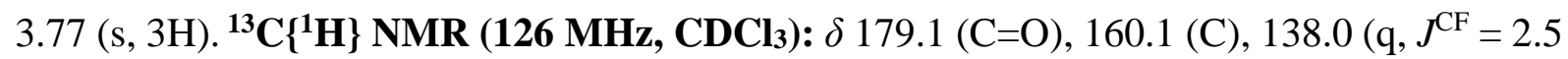
$\mathrm{Hz}, \mathrm{C}), 133.8(\mathrm{C}), 131.2(\mathrm{C}), 128.9(\mathrm{CH}), 128.6(2 \mathrm{CH}), 126.6\left(\mathrm{q}, J^{\mathrm{CF}}=4.2 \mathrm{~Hz}, \mathrm{CH}\right), 123.8(\mathrm{q}$, $\left.J^{\mathrm{CF}}=272.2 \mathrm{~Hz}, \mathrm{C}\right), 123.5(\mathrm{CH}), 114.3(2 \mathrm{CH}), 113.0\left(\mathrm{q}, J^{\mathrm{CF}}=33.4 \mathrm{~Hz}, \mathrm{C}\right), 76.9(\mathrm{C}), 55.4\left(\mathrm{CH}_{3}\right)$. ${ }^{19}$ F NMR (471 MHz, CDCl3): $\delta$-60.59. FT-IR (neat, $\mathbf{c m}^{-1}$ ): 3267, 2924, 1725, 1610, 1509, 1455, 1333, 1309, 1251, 1164. HRMS (ESI $\left.{ }^{+}\right): m / z$ calcd. for $\mathrm{C}_{16} \mathrm{H}_{12} \mathrm{NO}_{3} \mathrm{~F}_{3} \mathrm{Na}[\mathrm{M}+\mathrm{Na}]^{+}$ 346.0662 , found 346.0663 .

General procedure $\mathbf{C}$ was followed with $\mathbf{S 3 b}$ (250 mg, $0.770 \mathrm{mmol}$ ) Purification was performed with flash column chromatography over silica gel (40 g SiO 2 , heptane/EtOAc, 100/0 to 70/30, gradient) to afford $\mathbf{3 b}$ as a white solid (201 mg, $0.650 \mathrm{mmol}, 85 \%) .{ }^{\mathbf{1}} \mathbf{H}$ NMR (400 MHz, DMSO-d6): $\delta 10.96(\mathrm{~s}, 1 \mathrm{H}), 7.50(\mathrm{~d}, J=8.0 \mathrm{~Hz}, 1 \mathrm{H}), 7.29(\mathrm{~d}, J=7.4 \mathrm{~Hz}, 1 \mathrm{H}), 7.12(\mathrm{t}, J=7.7$ $\mathrm{Hz}, 1 \mathrm{H}), 7.06(\mathrm{~d}, J=8.6 \mathrm{~Hz}, 2 \mathrm{H}), 6.91(\mathrm{~d}, J=8.6 \mathrm{~Hz}, 2 \mathrm{H}), 4.82(\mathrm{~s}, 1 \mathrm{H}), 3.73(\mathrm{~s}, 3 \mathrm{H}) .{ }^{13} \mathbf{C}\left\{{ }^{1} \mathbf{H}\right\}$ NMR (100 MHz, DMSO-d6): $\delta 178.0$ (C=O), 158.6 (C), 139.9 (C), 132.4 (C), 129.5 (2CH), $128.7(\mathrm{CH}), 124.5\left(\mathrm{q}, J^{\mathrm{CF}}=4.5 \mathrm{~Hz}, \mathrm{CH}\right), 123.7\left(\mathrm{q}, J^{\mathrm{CF}}=271.5 \mathrm{~Hz}, \mathrm{C}\right), 121.8(\mathrm{CH}), 114.3(2 \mathrm{CH})$, $110.6\left(\mathrm{q}, J^{\mathrm{CF}}=33.0 \mathrm{~Hz}, \mathrm{C}\right), 55.1\left(\mathrm{CH}_{3}\right), 50.1(\mathrm{CH}) .{ }^{19}$ F NMR (565 MHz, DMSO): $\delta-59.98$. FT-IR (neat, $\mathbf{c m}^{-1}$ ): 3172, 3106, 2843, 1716, 1609, 1514, 1455, 1344, 1317, 1208, 1178. HRMS (ESI ${ }^{+}$): $m / z$ calcd. for $\mathrm{C}_{16} \mathrm{H}_{12} \mathrm{NO}_{2} \mathrm{~F}_{3} \mathrm{Na}[\mathrm{M}+\mathrm{Na}]^{+} 330.0713$, found 330.0713 .

\section{6-Methoxy-3-(4-methoxyphenyl)indolin-2-one 3c}

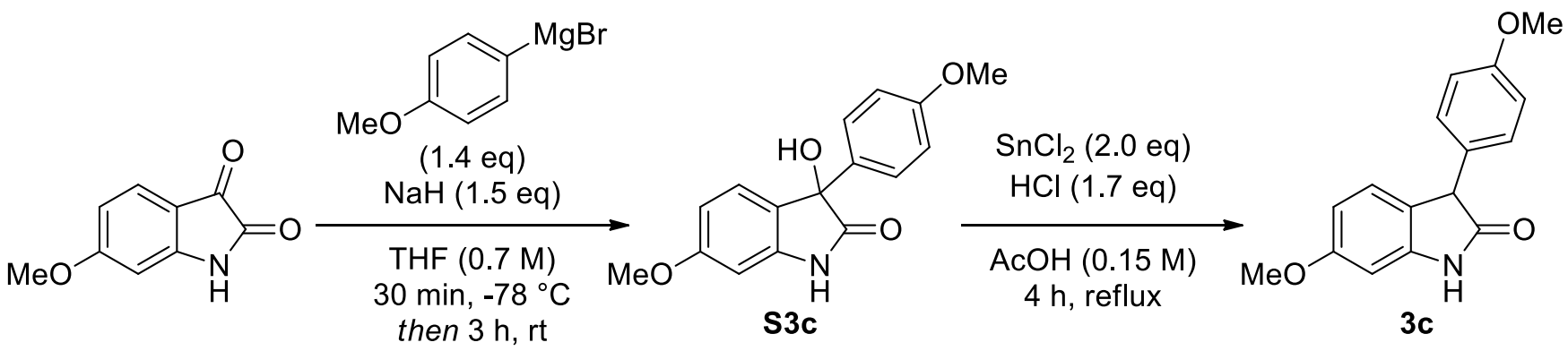

General procedure B was followed with 6-methoxyisatin (300 $\mathrm{mg}, 1.69 \mathrm{mmol}$ ) and bromoanisole ( $0.636 \mathrm{~mL}, 5.08 \mathrm{mmol}, 3.0$ equiv.) to afford S3c as a yellow solid $(260 \mathrm{mg}$, 0.910 mmol, 54\%). ${ }^{1} \mathbf{H}$ NMR (700 MHz, DMSO): $\delta 10.27$ (s, $\left.1 \mathrm{H}\right), 7.18(\mathrm{~d}, J=8.9 \mathrm{~Hz}, 2 \mathrm{H})$, $7.00(\mathrm{~d}, J=8.2 \mathrm{~Hz}, 1 \mathrm{H}), 6.86(\mathrm{~d}, J=8.9 \mathrm{~Hz}, 2 \mathrm{H}), 6.52(\mathrm{dd}, J=8.2,2.3 \mathrm{~Hz}, 1 \mathrm{H}), 6.44(\mathrm{~d}, J=$ $2.3 \mathrm{~Hz}, 1 \mathrm{H}), 6.40$ (s, 1H), 3.75 (s, 3H), 3.71 (s, 3H). ${ }^{13} \mathbf{C}\left\{{ }^{1} \mathbf{H}\right\}$ NMR (176 MHz, DMSO): $\delta$ 
$179.1(\mathrm{C}=\mathrm{O}), 160.3(\mathrm{C}), 158.6(\mathrm{C}), 142.2(\mathrm{C}), 133.8(\mathrm{C}), 126.9(2 \mathrm{CH}), 125.7(\mathrm{CH}), 125.6(\mathrm{C})$, $113.4(2 \mathrm{CH}), 106.8(\mathrm{CH}), 96.6(\mathrm{CH}), 76.6(\mathrm{C}), 55.3\left(\mathrm{CH}_{3}\right), 55.1\left(\mathrm{CH}_{3}\right)$. FT-IR (neat, $\left.\mathbf{c m}^{-1}\right)$ : 3301, 1723, 1630, 1606, 1507, 1463, 1350, 1298, 1253, 1181 HRMS (ESI $\left.{ }^{+}\right): m / z$ calcd. for $\mathrm{C}_{16} \mathrm{H}_{15} \mathrm{NO}_{4} \mathrm{Na}[\mathrm{M}+\mathrm{Na}]^{+}$308.0894, found 308.0890.

The general procedure C was followed with S3c $(250 \mathrm{mg}, 0.880 \mathrm{mmol})$ Purification was performed with flash column chromatography over silica gel (40 g SiO 2 , heptane/EtOAc, 100/0 to 70/30, gradient) to afford $\mathbf{3 c}$ as a light yellow solid (126 mg, $0.47 \mathrm{mmol}, 53 \%) .{ }^{1} \mathbf{H} \mathbf{~ N M R}$ (500 MHz, DMSO-d6): $\delta 10.43$ (s, 1H), 7.05 (d, $J=8.8 \mathrm{~Hz}, 2 \mathrm{H}), 6.93(\mathrm{~d}, J=8.5 \mathrm{~Hz}, 1 \mathrm{H}), 6.90$ $(\mathrm{d}, J=8.8 \mathrm{~Hz}, 2 \mathrm{H}), 6.52(\mathrm{dd}, J=8.2,2.4 \mathrm{~Hz}, 1 \mathrm{H}), 6.49$ (d, $J=2.3 \mathrm{~Hz}, 1 \mathrm{H}), 4.58(\mathrm{~s}, 1 \mathrm{H}), 3.75$ (s, 3H), 3.74 (s, 3H). ${ }^{13} \mathbf{C}\left\{{ }^{1} \mathbf{H}\right\}$ NMR (126 MHz, DMSO-d6): $\delta 178.1(\mathrm{C}=\mathrm{O}), 159.6(\mathrm{C}), 158.4$ (C), $143.9(\mathrm{C}), 130.0(\mathrm{C}), 129.3(2 \mathrm{CH}), 125.5(\mathrm{CH}), 122.2(\mathrm{C}), 114.1(2 \mathrm{CH}), 106.6(\mathrm{CH}), 96.4$ $(\mathrm{CH}), 55.3\left(\mathrm{CH}_{3}\right), 55.1\left(\mathrm{CH}_{3}\right), 50.5(\mathrm{CH})$. FT-IR (neat, $\left.\mathbf{c m}^{-1}\right)$ 2974, 2931, 2836, 1712, 1606, 1508, 1485, 1457, 1438, 1297, 1248, 1175. HRMS (ESI $\left.{ }^{+}\right): \mathrm{m} / z$ calcd. for $\mathrm{C}_{16} \mathrm{H}_{15} \mathrm{NO}_{3} \mathrm{Na}$ $[\mathrm{M}+\mathrm{Na}]^{+}$292.0945, found 292.0942 .

\section{5-Methoxy-3-(4-methoxyphenyl)indolin-2-one 3d}

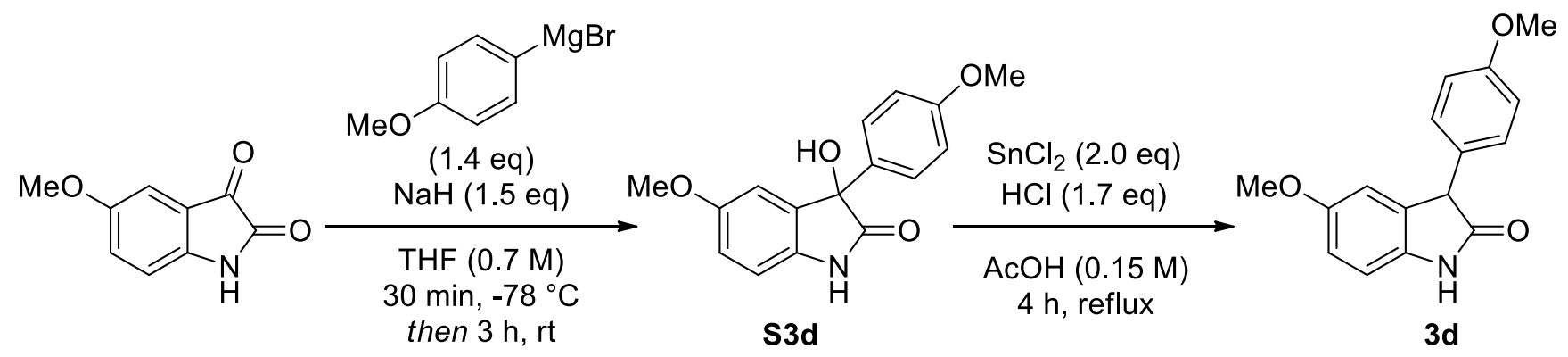

General procedure $\mathbf{B}$ was followed with 5-methoxylisatin (1.0 g, $5.64 \mathrm{mmol})$ and bromoanisole (1.41 mL, $11.3 \mathrm{mmol}, 2.0$ equiv.) to afford S3d as a brown solid (735 mg, $2.58 \mathrm{mmol}, 46 \%$ ). ${ }^{1}$ H NMR (400 MHz, DMSO-d6): $\delta 10.15$ (s, 1H), 7.18 (d, $\left.J=8.8 \mathrm{~Hz}, 2 \mathrm{H}\right), 6.86(\mathrm{~d}, J=8.8 \mathrm{~Hz}$, $2 \mathrm{H}), 6.81(\mathrm{~d}, J=2.5 \mathrm{~Hz}, 2 \mathrm{H}), 6.70(\mathrm{~d}, J=1.4 \mathrm{~Hz}, 1 \mathrm{H}), 6.52(\mathrm{~s}, 1 \mathrm{H}), 3.71(\mathrm{~s}, 3 \mathrm{H}), 3.66(\mathrm{~s}, 3 \mathrm{H})$. ${ }^{13} \mathbf{C}\left\{{ }^{1} \mathbf{H}\right\}$ NMR (100 MHz, DMSO-d6): $\delta 178.6(\mathrm{C}=\mathrm{O}), 158.7$ (C), 155.1 (C), $135.0(\mathrm{C}), 134.9$ (C), $133.5(\mathrm{C}), 126.8(2 \mathrm{CH}), 113.9(\mathrm{CH}), 113.5(2 \mathrm{CH}), 110.4(\mathrm{CH}), 110.3(\mathrm{CH}), 77.3(\mathrm{C}), 55.5$ $\left(\mathrm{CH}_{3}\right), 55.1\left(\mathrm{CH}_{3}\right)$. FT-IR (neat, $\left.\mathbf{c m}^{-1}\right)$ : 3261, 1716, 1681, 1607, 1509, 1485, 1464, 1440, 1301, 1256, 1182, 1147. HRMS (ESI $\left.{ }^{+}\right): m / z$ calcd. for $\mathrm{C}_{16} \mathrm{H}_{15} \mathrm{NO}_{4} \mathrm{Na}[\mathrm{M}+\mathrm{Na}]^{+} 308.0894$, found 308.0890 .

The general procedure C was followed with S3d (300 mg, $1.05 \mathrm{mmol}$ ) Purification was performed with flash column chromatography over silica gel (40 $\mathrm{g} \mathrm{SiO}_{2}$, heptane/EtOAc, 100/0 to 70/30, gradient) to afford 3d as a light orange solid (151 mg, $0.561 \mathrm{mmol}, 53 \%) .{ }^{1} \mathbf{H}$ NMR (600 MHz, DMSO-d6): $\delta 10.28$ (s, 1H), $7.05(\mathrm{~d}, J=8.7 \mathrm{~Hz}, 2 \mathrm{H}), 6.89$ (d, $J=8.7 \mathrm{~Hz}, 2 \mathrm{H}), 6.82-$ $6.77(\mathrm{~m}, 2 \mathrm{H}), 6.62(\mathrm{~s}, 1 \mathrm{H}), 4.63(\mathrm{~s}, 1 \mathrm{H}), 3.73(\mathrm{~s}, 3 \mathrm{H}), 3.65(\mathrm{~s}, 3 \mathrm{H}) .{ }^{13} \mathbf{C}\left\{{ }^{1} \mathbf{H}\right\}$ NMR (150 MHz, DMSO-d6): $\delta 177.3(\mathrm{C}=\mathrm{O}), 158.4(\mathrm{C}), 154.9(\mathrm{C}), 136.0(\mathrm{C}), 131.6(\mathrm{C}), 129.6(\mathrm{C}), 129.4(2 \mathrm{CH})$, $114.1(2 \mathrm{CH}), 112.8(\mathrm{CH}), 111.5(\mathrm{CH}), 109.8(\mathrm{CH}), 55.4\left(\mathrm{CH}_{3}\right), 55.1\left(\mathrm{CH}_{3}\right), 51.5(\mathrm{CH})$. FT-IR (neat, $\mathbf{~ c m}^{-1}$ ): 3160, 3043, 2839, 1693, 1602, 1513, 1484, 1454, 1441, 1207, 1178. HRMS $\left(\right.$ ESI $\left.^{+}\right): m / z$ calcd. for $\mathrm{C}_{16} \mathrm{H}_{15} \mathrm{NO}_{3} \mathrm{Na}[\mathrm{M}+\mathrm{Na}]^{+} 292.0945$, found 292.0943 . 
<smiles>COc1ccc(C2(O)c3cc(Br)ccc3NC(=O)C2(O)c2cc(C3C(=O)Nc4ccc(Br)cc43)ccc2OC)cc1</smiles>

General procedure $\mathbf{B}$ was followed with 5-bromoisatin $(3.0 \mathrm{~g}, 13.3 \mathrm{mmol})$ and bromoanisole (2.16 mL, $17.3 \mathrm{mmol}, 1.3$ equiv.) to afford S3e as a light yellow solid (3.6 $\mathrm{mg}, 10.9 \mathrm{mmol}$, 82\%). ${ }^{1}$ H NMR (700 MHz, DMSO-d6): $\delta 10.50$ (s, 1H), 7.43 (dd, $\left.J=8.3,2.1 \mathrm{~Hz}, 1 \mathrm{H}\right), 7.21$ (t, $J=4.4 \mathrm{~Hz}, 1 \mathrm{H}), 7.20-7.17(\mathrm{~m}, 2 \mathrm{H}), 6.91-6.87(\mathrm{~m}, 2 \mathrm{H}), 6.86$ (dd, $J=8.6,3.4 \mathrm{~Hz}, 1 \mathrm{H}), 6.68$ (s, 1H), $3.72(\mathrm{~s}, 3 \mathrm{H}) .{ }^{13} \mathbf{C}\left\{{ }^{1} \mathbf{H}\right\}$ NMR (150 MHz, CDCl$): ~ \delta 178.2(\mathrm{C}=\mathrm{O}), 158.8(\mathrm{C}), 141.2(\mathrm{C})$, $136.2(\mathrm{C}), 132.8(\mathrm{C}), 131.9(\mathrm{CH}), 127.4(\mathrm{CH}), 126.7(2 \mathrm{CH}), 113.7(2 \mathrm{CH}), 113.6(\mathrm{C}), 112.0$ (CH), $76.9(\mathrm{C}), 55.1\left(\mathrm{CH}_{3}\right)$. FT-IR (neat, $\mathbf{c m}^{-\mathbf{1}}$ ): 3268, 3178, 1716, 1698, 1672, 1608, 1509, 1473, 1441, 1255, 1170, 1127. HRMS (ESI $\left.{ }^{+}\right): m / z$ calcd. for $\mathrm{C}_{15} \mathrm{H}_{12} \mathrm{BrNO}_{3} \mathrm{Na}[\mathrm{M}+\mathrm{Na}]^{+}$ 355.9893 , found 355.9892 .

The general procedure C was followed with S3e $(175 \mathrm{mg}, 0.524 \mathrm{mmol})$ Purification was performed with flash column chromatography over silica gel (40 g SiO 2 , heptane/EtOAc, 100/0 to 70/30, gradient) to afford $\mathbf{3 e}$ as a white solid (140 mg, $0.44 \mathrm{mmol}, 84 \%)$. ${ }^{1} \mathbf{H}$ NMR (600 MHz, CDCl 3$): \delta 8.43(\mathrm{~s}, 1 \mathrm{H}), 7.39-7.36(\mathrm{~m}, 1 \mathrm{H}), 7.24(\mathrm{~s}, 1 \mathrm{H}), 7.11(\mathrm{~d}, J=8.7 \mathrm{~Hz}, 2 \mathrm{H}), 6.89$ $(\mathrm{d}, J=8.7 \mathrm{~Hz}, 2 \mathrm{H}), 6.81(\mathrm{~d}, J=8.3 \mathrm{~Hz}, 1 \mathrm{H}), 4.57(\mathrm{~s}, 1 \mathrm{H}), 3.80(\mathrm{~s}, 3 \mathrm{H}),{ }^{13} \mathbf{C}\left\{{ }^{1} \mathbf{H}\right\} \mathbf{N M R}$ (150 MHz, CDCl $)$ : $\delta 178.2(\mathrm{C}=\mathrm{O}), 159.2(\mathrm{C}), 140.6(\mathrm{C}), 132.1(\mathrm{C}), 131.4(\mathrm{CH}), 129.6(2 \mathrm{CH})$, $128.6(\mathrm{CH}), 127.7(\mathrm{C}), 115.5(\mathrm{C}), 114.7(2 \mathrm{CH}), 111.4(\mathrm{CH}), 55.5\left(\mathrm{CH}_{3}\right), 52.0(\mathrm{CH})$. FT-IR (neat, $\mathbf{~ c m}^{-1}$ ): $3184,1710,1609,1509,1473,1249,1216,1177$. HRMS (ESI ${ }^{+}$): $\mathrm{m} / z$ calcd. for $\mathrm{C}_{15} \mathrm{H}_{12} \mathrm{NO}_{2} \mathrm{BrNa}[\mathrm{M}+\mathrm{Na}]^{+}$339.9944, found 339.9940.

\section{3-(4-Methoxyphenyl)-5-nitroindolin-2-one 3f}<smiles>COc1ccc(C2C(=O)Nc3ccc([N+](=O)[O-])cc32)cc1</smiles>

The general procedure B was followed with 5-nitroisatin (500 mg, $2.60 \mathrm{mmol}$ ) and 4-bromoanisole $(0.42 \mathrm{~mL}, 3.38 \mathrm{mmol}, 1.3$ equiv.) to afford $\mathbf{S 3 f}$ as a white solid $(541 \mathrm{mg}$, 1.80 mmol, 69\%). ${ }^{1} \mathbf{H}$ NMR (500 MHz, DMSO-d6): $\delta 8.24$ (dd, $\left.J=8.6,2.4 \mathrm{~Hz}, 1 \mathrm{H}\right), 7.90$ (d, $J=2.4 \mathrm{~Hz}, 1 \mathrm{H}), 7.23(\mathrm{~d}, J=8.9 \mathrm{~Hz}, 2 \mathrm{H}), 7.11(\mathrm{~d}, J=8.6 \mathrm{~Hz}, 1 \mathrm{H}), 6.92-6.88(\mathrm{~m}, 3 \mathrm{H}), 3.72(\mathrm{~s}$, 3H). ${ }^{13} \mathbf{C}\left\{{ }^{1} \mathbf{H}\right\}$ NMR (126 MHz, DMSO-d6): $\delta 178.8(\mathrm{C}=\mathrm{O}), 159.1(\mathrm{C}), 148.5(\mathrm{C}), 142.5(\mathrm{C})$, $134.6(\mathrm{C}), 132.0(\mathrm{C}), 126.8(2 \mathrm{CH}), 126.6(\mathrm{CH}), 120.2(\mathrm{CH}), 113.8(2 \mathrm{CH}), 110.4(\mathrm{CH}), 76.5$ 
(C), $55.2\left(\mathrm{CH}_{3}\right)$. FT-IR (neat, $\left.\mathbf{c m}^{-\mathbf{1}}\right)$ : 3285, 1723, 1608, 1521, 1509, 1458, 1344, 1302, 1256, 1176, 1123. HRMS (ESI $\left.{ }^{+}\right): m / z$ calcd. for $\mathrm{C}_{15} \mathrm{H}_{12} \mathrm{~N}_{2} \mathrm{O}_{5} \mathrm{Na}[\mathrm{M}+\mathrm{Na}]^{+} 323.0639$, found 323.0639.

The general procedure C was followed with S3f (300 mg, $1.00 \mathrm{mmol}$ ) Purification was performed with flash column chromatography over silica gel (40 g SiO 2 , heptane/EtOAc, 100/0 to 70/30, gradient) to afford $\mathbf{3 f}$ as a brown solid (120 mg, $0.42 \mathrm{mmol}, 42 \%)$. ${ }^{\mathbf{1}} \mathbf{H}$ NMR (600 MHz, CDCl3): $\delta 9.15(\mathrm{~s}, 1 \mathrm{H}), 8.23(\mathrm{dd}, J=8.6,1.6 \mathrm{~Hz}, 1 \mathrm{H}), 8.03(\mathrm{~s}, 1 \mathrm{H}), 7.11(\mathrm{~d}, J=8.6 \mathrm{~Hz}$, 2H), $7.03(\mathrm{~d}, J=8.6 \mathrm{~Hz}, 1 \mathrm{H}), 6.91(\mathrm{~d}, J=8.7 \mathrm{~Hz}, 2 \mathrm{H}), 4.66(\mathrm{~s}, 1 \mathrm{H}), 3.80(\mathrm{~s}, 3 \mathrm{H}) .{ }^{13} \mathbf{C}\left\{{ }^{1} \mathbf{H}\right\} \mathbf{N M R}$ (151 MHz, CDCl 3 ) $\delta 177.95$ (C=O), 158.77 (C), 146.39 (C), 142.90 (C), 129.88 (C), 128.60 $(\mathrm{CH}), 125.65(\mathrm{C}), 124.73(\mathrm{CH}), 120.38(\mathrm{CH}), 113.93(\mathrm{CH}), 108.88(\mathrm{CH}), 54.50\left(\mathrm{CH}_{3}\right), 50.80$ (CH). FT-IR (neat, cm-1 ): 3128, 3080, 2931, 1712, 1621, 1605, 1509, 1456, 1337, 1298, 1183. HRMS (ESI ${ }^{+}$): $m / z$ calcd. for $\mathrm{C}_{15} \mathrm{H}_{12} \mathrm{~N}_{2} \mathrm{O}_{4} \mathrm{Na}[\mathrm{M}+\mathrm{Na}]^{+}$307.0690, found 307.0693 .

\section{1,3-Dimethylindolin-2-one 5a}

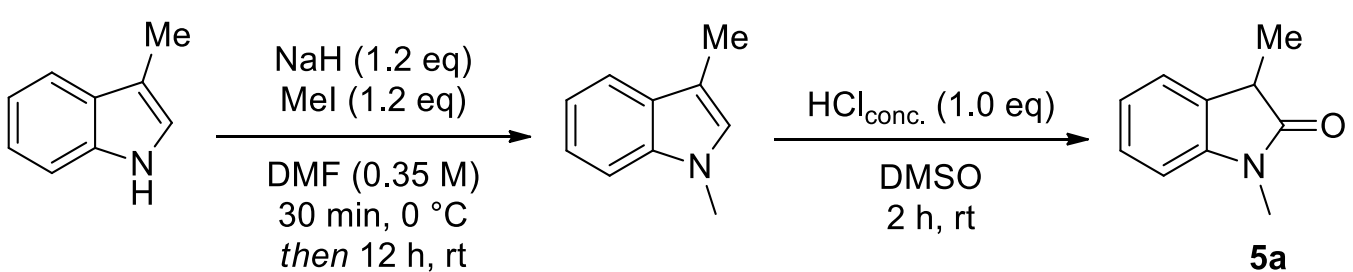

Following a modified reported procedure. ${ }^{11}$ To a stirring solution of $\mathrm{NaH}(60 \%$ in mineral oil, $180 \mathrm{mg}, 4.57 \mathrm{mmol}, 1.2$ equiv.) in dry DMF (10 mL), 3-methylindole (500 mg, $3.81 \mathrm{mmol}$, 1.0 equiv.) was added dropwise at $0{ }^{\circ} \mathrm{C}$. The mixture was allowed to warm to $\mathrm{rt}$ and stirred for $30 \mathrm{~min}$. After cooling to $0{ }^{\circ} \mathrm{C}$, MeI $(0.29 \mathrm{~mL}, 4.57 \mathrm{mmol}, 1.2$ equiv. $)$ was added dropwise. The resulting mixture was stirred at $\mathrm{rt}$ for $12 \mathrm{~h}$, then quenched with $\mathrm{H}_{2} \mathrm{O}$ and extracted with ethyl acetate. The combined organic phases were dried over $\mathrm{MgSO}_{4}$, filtered, and concentrated under vacuum. The crude product was used directly in the next step without purification.

Following a reported procedure, ${ }^{12}$ to a solution of $\mathrm{N}$-methyl-3-methylindole $(639 \mathrm{mg}$, $4.40 \mathrm{mmol}, 1.0$ equiv.) in DMSO $(20 \mathrm{~mL})$ was added slowly concentrated $\mathrm{HCl}(17.6 \mathrm{~mL}$, $4.40 \mathrm{mmol}, 1.0$ equiv.) at rt. After stirring for $2 \mathrm{~h}$, the solution was diluted with $\mathrm{H}_{2} \mathrm{O}(20 \mathrm{~mL})$ and extracted with ethyl acetate. The combined organic layers were washed with sat. $\mathrm{NaHCO}_{3}$ $(30 \mathrm{~mL})$, brine $(30 \mathrm{~mL})$, dried over anhydrous $\mathrm{MgSO}_{4}$ and concentrated in vacuo. The residue was purified by $\mathrm{FC}\left(20 \mathrm{~g} \mathrm{SiO}_{2}\right.$, DCM/ethyl acetate: $100 / 0$ to $95 / 5,10 \mathrm{CV}$ ) to provide $\mathbf{5 a}$ as an orange oil (366 mg, $2.27 \mathrm{mmol}, 52 \%$ ).

${ }^{1}$ H NMR (400 MHz, CDCl $): \delta 7.29(\mathrm{dd}, J=8.2,7.3 \mathrm{~Hz}, 1 \mathrm{H}), 7.25(\mathrm{~d}, J=7.4 \mathrm{~Hz}, 1 \mathrm{H}), 7.07$ $(\mathrm{t}, J=7.5 \mathrm{~Hz}, 1 \mathrm{H}), 6.84(\mathrm{~d}, J=7.8 \mathrm{~Hz}, 1 \mathrm{H}), 3.44(\mathrm{q}, J=7.7 \mathrm{~Hz}, 1 \mathrm{H}), 3.22(\mathrm{~s}, 3 \mathrm{H}), 1.49$ (d, $J=$ $7.7 \mathrm{~Hz}, 3 \mathrm{H})$. Spectral and physical data are in accordance with literature. ${ }^{13}$ 


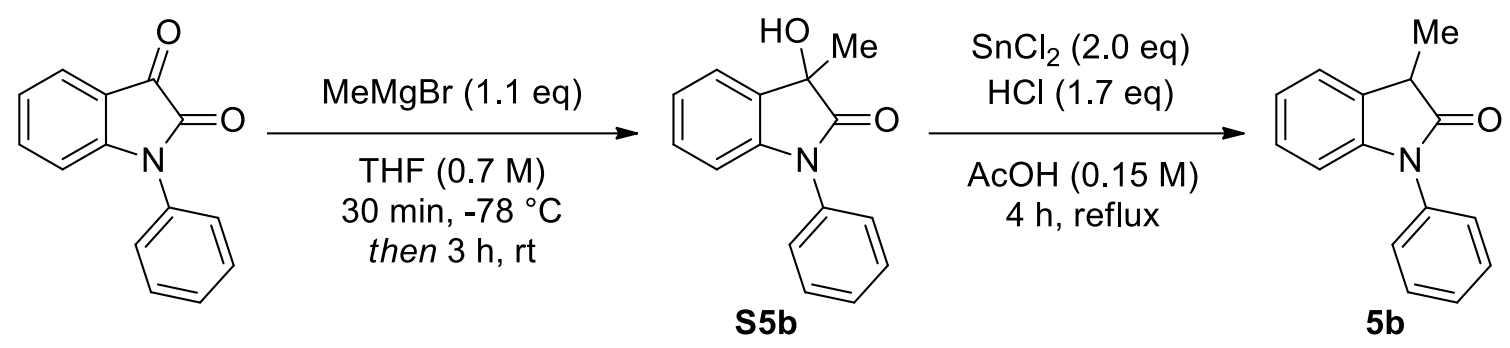

The general procedure B was followed with 1-phenylisatin (500 $\mathrm{mg}, 2.24 \mathrm{mmol}$ ) and methylmagnesiumbromide ( $3 \mathrm{M}$ in $\mathrm{Et}_{2} \mathrm{O}, 0.82 \mathrm{~mL}, 1.83 \mathrm{mmol}, 1.1$ equiv.) to afford $\mathbf{S 5 b}$ as a light yellow solid (437 mg, $1.83 \mathrm{mmol}, 82 \%)$. ${ }^{1} \mathbf{H}$ NMR (500 MHz, DMSO-d6): $\delta$ 7.60-7.55 $(\mathrm{m}, 2 \mathrm{H}), 7.48-7.43(\mathrm{~m}, 2 \mathrm{H}), 7.43-7.39(\mathrm{~m}, 2 \mathrm{H}), 7.25(\mathrm{td}, J=7.7,1.3 \mathrm{~Hz}, 1 \mathrm{H}), 7.11(\mathrm{td}, J=7.5$, $0.8 \mathrm{~Hz}, 1 \mathrm{H}), 6.72(\mathrm{~d}, J=7.8 \mathrm{~Hz}, 1 \mathrm{H}), 6.15(\mathrm{~s}, 1 \mathrm{H}), 1.51(\mathrm{~s}, 3 \mathrm{H}) .{ }^{13} \mathbf{C}\left\{{ }^{1} \mathbf{H}\right\}$ NMR $(\mathbf{1 2 6} \mathbf{~ M H z}$, DMSO-d6): $\delta 177.4(\mathrm{C}=\mathrm{O}), 142.3(\mathrm{C}), 134.3(\mathrm{C}), 132.9(\mathrm{C}), 129.7(2 \mathrm{CH}), 129.0(\mathrm{CH}), 128.0$ $(\mathrm{CH}), 126.6(2 \mathrm{CH}), 123.7(\mathrm{CH}), 123.1(\mathrm{CH}), 109.0(\mathrm{CH}), 72.5(\mathrm{C}), 24.7\left(\mathrm{CH}_{3}\right) . \mathbf{H R M S}\left(\mathbf{E S I}^{+}\right)$: $m / z$ calcd. for $\mathrm{C}_{15} \mathrm{H}_{13} \mathrm{NO}_{2} \mathrm{Na}[\mathrm{M}+\mathrm{Na}]^{+} 262.0839$, found 262.0838. Spectral and physical data are in accordance with literature. ${ }^{14}$

The general procedure C was followed with S5b (250 mg, $1.04 \mathrm{mmol}$ ) Purification was performed with flash column chromatography over silica gel (40 g SiO 2 , heptane/EtOAc, 100/0 to 70/30, gradient) to afford $\mathbf{5 b}$ as a colorless oil (200 $\mathrm{mg}, 0.896 \mathrm{mmol}, 86 \%)$. ${ }^{\mathbf{1}} \mathbf{H}$ NMR (600 MHz, CDCl3): $\delta 7.53(\mathrm{t}, J=7.8 \mathrm{~Hz}, 2 \mathrm{H}), 7.44-7.39(\mathrm{~m}, 3 \mathrm{H}), 7.31(\mathrm{~d}, J=7.3 \mathrm{~Hz}, 1 \mathrm{H}), 7.20$ (t, $J=7.7 \mathrm{~Hz}, 1 \mathrm{H}), 7.10(\mathrm{t}, J=7.5 \mathrm{~Hz}, 1 \mathrm{H}), 6.82(\mathrm{~d}, J=7.9 \mathrm{~Hz}, 1 \mathrm{H}), 3.63(\mathrm{~d}, J=7.6 \mathrm{~Hz}, 1 \mathrm{H}), 1.60$ $(\mathrm{d}, J=7.6 \mathrm{~Hz}, 3 \mathrm{H}) .{ }^{13} \mathbf{C}\left\{{ }^{1} \mathbf{H}\right\}$ NMR (150 MHz, CDCl 3$): ~ \delta 178.1(\mathrm{C}=\mathrm{O}), 144.1(\mathrm{C}), 134.7(\mathrm{C})$, $130.6(\mathrm{C}), 129.7(2 \mathrm{CH}), 128.1(\mathrm{CH}), 127.9(\mathrm{CH}), 126.7(2 \mathrm{CH}), 123.9(\mathrm{CH}), 123.0(\mathrm{CH}), 109.4$ $(\mathrm{CH}), 40.9(\mathrm{CH}), 15.8\left(\mathrm{CH}_{3}\right)$. HRMS $\left(\mathbf{E S I}^{+}\right): \mathrm{m} / z$ calcd. for $\mathrm{C}_{15} \mathrm{H}_{13} \mathrm{NONa}[\mathrm{M}+\mathrm{Na}]^{+} 246.0890$, found 246.0892. Spectral and physical data are in accordance with literature. ${ }^{13}$

\section{Tert-butyl 2-oxo-3-phenylindoline-1-carboxylate 5c}

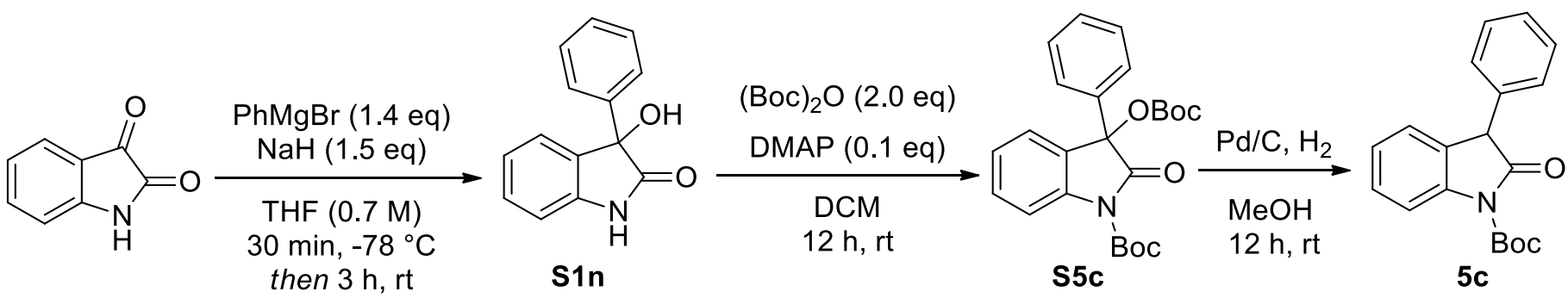

Following reported procedure, ${ }^{14} \mathbf{S 1 n}(500 \mathrm{mg}, 2.22 \mathrm{mmol})$ was dissolved in DCM (22 mL). To this solution were added DMAP $(27.1 \mathrm{mg}, 0.220 \mathrm{mmol})$ and (Boc) $)_{2} \mathrm{O}(969 \mathrm{mg}, 4.44 \mathrm{mmol})$ at $\mathrm{rt}$, and then the mixture was stirred for $12 \mathrm{~h}$. The reaction mixture was diluted with ethyl acetate, and then quenched with saturated aqueous $\mathrm{NH}_{4} \mathrm{Cl}$. The aqueous layer was extracted with ethyl acetate, and the combined organic layers were washed with $\mathrm{H}_{2} \mathrm{O}$ and brine and then dried over $\mathrm{MgSO}_{4}$. After the removal of solvent, purification by FC (gradient, heptane/ethyl acetate, 010\%) gave S5c (852 mg, $1.99 \mathrm{mmol}, 90 \%)$ as a white solid. ${ }^{\mathbf{1}} \mathbf{H}$ NMR (700 MHz, CDCl $\left.\mathbf{3}\right): \delta$ $7.99(\mathrm{~d}, J=8.2 \mathrm{~Hz}, 1 \mathrm{H}), 7.45(\mathrm{td}, J=8.2,1.4 \mathrm{~Hz}, 1 \mathrm{H}), 7.35-7.29(\mathrm{~m}, 6 \mathrm{H}), 7.24(\mathrm{td}, J=7.5,0.7$ $\mathrm{Hz}, 1 \mathrm{H}), 1.61$ (s, 9H), 1.38 (s, 9H). ${ }^{13} \mathbf{C}\left\{{ }^{1} \mathbf{H}\right\}$ NMR (176 MHz, CDCl$): \delta 171.7(\mathrm{C}=\mathrm{O}), 151.1$ $(\mathrm{C}=\mathrm{O}), 149.2(\mathrm{C}=\mathrm{O}), 140.8(\mathrm{C}), 136.1(\mathrm{C}), 130.5(\mathrm{CH}), 129.3(\mathrm{CH}), 128.7(2 \mathrm{CH}), 127.5(\mathrm{C})$, 
$126.9(2 \mathrm{CH}), 125.1(\mathrm{CH}), 124.2(\mathrm{CH}), 115.6(\mathrm{CH}), 84.7(\mathrm{C}), 84.1(\mathrm{C}), 81.8(\mathrm{C}), 28.2\left(3 \mathrm{CH}_{3}\right)$, $27.7\left(3 \mathrm{CH}_{3}\right) . \mathbf{H R M S}\left(\mathbf{E S I}^{+}\right): \mathrm{m} / z$ calcd. for $\mathrm{C}_{24} \mathrm{H}_{27} \mathrm{NO}_{6} \mathrm{Na}[\mathrm{M}+\mathrm{Na}]^{+} 448.1731$, found 448.1730 .

S5c $(850 \mathrm{mg}, 1.99 \mathrm{mmol})$ was dissolved in $\mathrm{MeOH}(9 \mathrm{~mL}) . \mathrm{Pd} / \mathrm{C}(423 \mathrm{mg}, 0.400 \mathrm{mmol}$, 0.20 equiv.) was added to this solution, and the resulting mixture was stirred under hydrogen atmosphere (balloon) for $12 \mathrm{~h}$ at $\mathrm{rt}$. The reaction mixture was filtered through celite to remove $\mathrm{Pd} / \mathrm{C}$, and the residue was washed with $\mathrm{Et}_{2} \mathrm{O}$. After the removal of solvent, the crude product was purified by FC (gradient, heptane/ethyl acetate, 0-15\%) to give 5c (300 $\mathrm{mg}, 0.97 \mathrm{mmol}$, 56\%) as a white solid. ${ }^{1} \mathbf{H}$ NMR (700 MHz, $\left.\mathbf{C D C l}_{3}\right): \delta 7.93(\mathrm{~d}, J=8.2 \mathrm{~Hz}, 1 \mathrm{H}), 7.38-7.33$ (m, $3 \mathrm{H}), 7.32-7.29(\mathrm{~m}, 1 \mathrm{H}), 7.21-7.19(\mathrm{~m}, 2 \mathrm{H}), 7.18-7.15(\mathrm{~m}, 1 \mathrm{H}), 4.73(\mathrm{~s}, 1 \mathrm{H}), 1.63(\mathrm{~s}, 9 \mathrm{H})$. ${ }^{13} \mathbf{C}\left\{{ }^{1} \mathbf{H}\right\}$ NMR (175 MHz, CDCl 3$): \delta 174.0(\mathrm{C}=\mathrm{O}), 149.5(\mathrm{C}=\mathrm{O}), 140.6(\mathrm{C}), 136.4(\mathrm{C}), 129.0$ $(2 \mathrm{CH}), 128.8(3 \mathrm{CH}), 128.0(\mathrm{CH}), 127.5(\mathrm{C}), 125.2(\mathrm{CH}), 124.7(\mathrm{CH}), 115.2(\mathrm{CH}), 84.5(\mathrm{C})$, $52.7(\mathrm{CH}), 28.2\left(3 \mathrm{CH}_{3}\right)$. HRMS $\left(\mathbf{E S I}^{+}\right): \mathrm{m} / \mathrm{z}$ calcd. for $\mathrm{C}_{19} \mathrm{H}_{19} \mathrm{NO}_{3} \mathrm{Na}[\mathrm{M}+\mathrm{Na}]^{+} 332.1258$, found 332.1256 . Spectral and physical data are in accordance with literature. ${ }^{14}$ 


\section{Characterization data of 3,3-substituted oxindoles 2a-s, 4a-f, 6a-c and 7a-i}

\section{3-Ethoxy-3-methylindolin-2-one 2a}<smiles>CCOC1(C)C(=O)Nc2ccccc21</smiles>

$2 a$

General procedure A was followed with 3-methyloxindole $(61.3 \mathrm{mg}, 0.40 \mathrm{mmol})$ and ethanol $(2.5 \mathrm{~mL})$. Purification was performed with flash column chromatography over silica gel (20 g $\mathrm{SiO}_{2}$, heptane/EtOAc, 100/0 to 50/50, gradient) to afford 2a as a light yellow solid (43.3 mg, 0.226 mmol, 57\%). ${ }^{1} \mathbf{H}$ NMR (600 MHz, CDCl3): $\delta 8.88$ (brs, $\left.1 \mathrm{H}\right), 7.32(\mathrm{~d}, J=7.3 \mathrm{~Hz}, 1 \mathrm{H})$, 7.29-7.26 (m, 1H), 7.09 (t, $J=7.5 \mathrm{~Hz}, 1 \mathrm{H}), 6.94(\mathrm{~d}, J=7.7 \mathrm{~Hz}, 1 \mathrm{H}), 3.28(\mathrm{tt}, J=14.0,7.0 \mathrm{~Hz}$, $1 \mathrm{H}), 3.13(\mathrm{dq}, J=14.0,7.0 \mathrm{~Hz}, 1 \mathrm{H}), 1.59(\mathrm{~s}, 3 \mathrm{H}), 1.16(\mathrm{t}, J=7.0 \mathrm{~Hz}, 3 \mathrm{H}) .{ }^{13} \mathbf{C}\left\{{ }^{1} \mathbf{H}\right\} \mathbf{~ N M R}$ (150 MHz, CDCl3): $\delta 179.7(\mathrm{C}=\mathrm{O}), 140.6(\mathrm{C}), 129.9(\mathrm{C}), 129.7(\mathrm{CH}), 124.1(\mathrm{CH}), 123.2(\mathrm{CH})$, $110.6(\mathrm{CH}), 79.7(\mathrm{C}), 61.3\left(\mathrm{CH}_{2}\right), 24.4\left(\mathrm{CH}_{3}\right), 15.5\left(\mathrm{CH}_{3}\right)$. FT-IR (neat, $\left.\mathbf{c m}^{-1}\right)$ : 3229, 2976 , 2927, 1714, 1618, 1605, 1470, 1204, 1128. HRMS (ESI $\left.{ }^{+}\right): \mathrm{m} / z$ calcd. for $\mathrm{C}_{11} \mathrm{H}_{13} \mathrm{NO}_{2} \mathrm{Na}$ $[\mathrm{M}+\mathrm{Na}]^{+} 214.0838$, found 214.0840 .

\section{3-Ethoxyindolin-2-one $2 \mathrm{~b}$}<smiles>CCOC1C(=O)Nc2ccccc21</smiles>

2b

General procedure A was followed with oxindole $(53.3 \mathrm{mg}, 0.40 \mathrm{mmol})$ and ethanol $(2.5 \mathrm{~mL})$. Purification was performed with flash column chromatography over silica gel $\left(20 \mathrm{~g} \mathrm{SiO}_{2}\right.$, heptane/EtOAc, 100/0 to 50/50, gradient) to afford $\mathbf{2 b}$ as a light yellow solid (7.3 mg,

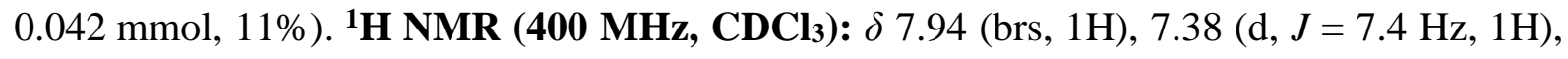
7.30-7.24 (m, 1H), $7.06(\mathrm{t}, J=7.4 \mathrm{~Hz}, 1 \mathrm{H}), 6.85(\mathrm{~d}, J=7.8 \mathrm{~Hz}, 1 \mathrm{H}), 4.93(\mathrm{~s}, 1 \mathrm{H}), 3.88(\mathrm{dq}, J=$ 14.1, 7.0 Hz, 1H), $3.70(\mathrm{dq}, J=14.1,7.0 \mathrm{~Hz}, 1 \mathrm{H}), 1.29(\mathrm{t}, J=7.0 \mathrm{~Hz}, 3 \mathrm{H}) .{ }^{13} \mathbf{C}\left\{{ }^{1} \mathbf{H}\right\} \mathbf{~ N M R}$ (100 MHz, CDCl $)$ : $\delta 176.8(\mathrm{C}=\mathrm{O}), 141.3(\mathrm{C}), 130.0(\mathrm{CH}), 125.9(\mathrm{C}), 125.8(\mathrm{CH}), 123.1(\mathrm{CH})$, $110.2(\mathrm{CH}), 76.1(\mathrm{CH}), 64.6\left(\mathrm{CH}_{2}\right), 15.5\left(\mathrm{CH}_{3}\right)$. FT-IR (neat, $\left.\mathbf{c m}^{-1}\right)$ : 3132, 3083, 2961, 2926, 1703, 1617, 1470, 1259. HRMS (ESI $\left.{ }^{+}\right): \mathrm{m} / z$ calcd. for $\mathrm{C}_{10} \mathrm{H}_{11} \mathrm{NO}_{2} \mathrm{Na}[\mathrm{M}+\mathrm{Na}]^{+} 200.0682$, found 200.0678 . 
<smiles>CCOC1(C(C)C)C(=O)Nc2ccccc21</smiles>

General procedure A was followed with 1c $(70.1 \mathrm{mg}, 0.40 \mathrm{mmol})$ and ethanol $(2.5 \mathrm{~mL})$. Purification was performed with flash column chromatography over silica gel $\left(20 \mathrm{~g} \mathrm{SiO}_{2}\right.$, heptane/EtOAc, 100/0 to 50/50, gradient) to afford $\mathbf{2 c}$ as a yellow solid (25.0 $\mathrm{mg}, 0.114 \mathrm{mmol}$, 29\%). ${ }^{1} \mathbf{H}$ NMR (400 MHz, CDCl3): $\delta 8.02$ (brs, 1H), 7.32-7.24 (m, 2H), 7.06 (t, $J=7.5 \mathrm{~Hz}$, $1 \mathrm{H}), 6.87(\mathrm{~d}, J=7.7 \mathrm{~Hz}, 1 \mathrm{H}), 3.26(\mathrm{dq}, J=14.0,7.0 \mathrm{~Hz}, 1 \mathrm{H}), 3.10(\mathrm{dq}, J=14.0,7.0 \mathrm{~Hz}, 1 \mathrm{H})$, 2.35-2.25 (m, $1 \mathrm{H}), 1.14(\mathrm{t}, J=7.0 \mathrm{~Hz}, 3 \mathrm{H}), 1.04(\mathrm{~d}, J=6.8 \mathrm{~Hz}, 3 \mathrm{H}), 0.79(\mathrm{~d}, J=6.8 \mathrm{~Hz}, 3 \mathrm{H})$. ${ }^{13} \mathbf{C}\left\{{ }^{1} \mathbf{H}\right\}$ NMR (100 MHz, $\left.\mathbf{C D C l}_{3}\right): \delta 178.9(\mathrm{C}=\mathrm{O}), 141.4(\mathrm{C}), 129.5(\mathrm{CH}), 127.3(\mathrm{C}), 125.4$ $(\mathrm{CH}), 122.7(\mathrm{CH}), 110.0(\mathrm{CH}), 85.8(\mathrm{C}), 61.2\left(\mathrm{CH}_{2}\right), 35.8(\mathrm{CH}), 16.2\left(\mathrm{CH}_{3}\right), 16.2\left(\mathrm{CH}_{3}\right), 15.6$ $\left(\mathrm{CH}_{3}\right)$. FT-IR (neat, $\left.\mathbf{c m}^{-1}\right)$ : 3152, 3109, 2966, 2923, 1733, 1687, 1617, 1466, 1213. HRMS $\left(\mathbf{E S I}^{+}\right): m / z$ calcd. for $\mathrm{C}_{13} \mathrm{H}_{17} \mathrm{NO}_{2} \mathrm{Na}[\mathrm{M}+\mathrm{Na}]^{+} 242.1151$, found 242.1152 .

\section{3-Benzyl-3-ethoxyindolin-2-one 2d}<smiles>CCOC1(Cc2ccccc2)C(=O)Nc2ccccc21</smiles>

2d

General procedure A was followed with 1d $(89.3 \mathrm{mg}, 0.40 \mathrm{mmol})$ and ethanol $(2.5 \mathrm{~mL})$. Purification was performed with flash column chromatography over silica gel $\left(20 \mathrm{~g} \mathrm{SiO}_{2}\right.$, heptane/EtOAc, 100/0 to 50/50, gradient) to afford 2d as a light yellow solid $(35.0 \mathrm{mg}$,

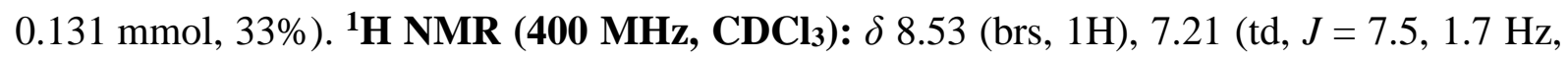
$1 \mathrm{H}), 7.13-7.01(\mathrm{~m}, 5 \mathrm{H}), 6.96-6.92(\mathrm{~m}, 2 \mathrm{H}), 6.75(\mathrm{dd}, J=7.6,3.7 \mathrm{~Hz}, 1 \mathrm{H}), 3.22(\mathrm{~d}, J=12.9 \mathrm{~Hz}$, 1H), 3.29-3.20 (m, 2H), $3.13(\mathrm{dd}, J=13.8,3.7 \mathrm{~Hz}, 1 \mathrm{H}), 1.17(\mathrm{t}, J=7.0 \mathrm{~Hz}, 3 \mathrm{H}) .{ }^{13} \mathbf{C}\left\{{ }^{1} \mathbf{H}\right\} \mathbf{~ N M R}$ (100 MHz, CDCl3): $\delta 178.8(\mathrm{C}=\mathrm{O}), 141.1(\mathrm{C}), 134.1(\mathrm{C}), 130.7(2 \mathrm{CH}), 129.8(\mathrm{CH}), 127.7$ $(2 \mathrm{CH}), 127.4(\mathrm{C}), 126.8(\mathrm{CH}), 125.3(\mathrm{CH}), 122.7(\mathrm{CH}), 110.3(\mathrm{CH}), 83.7(\mathrm{C}), 61.4\left(\mathrm{CH}_{2}\right), 44.0$ $\left(\mathrm{CH}_{2}\right), 15.5\left(\mathrm{CH}_{3}\right)$. HRMS (ESI $\left.)^{+}\right): \mathrm{m} / z$ calcd. for $\mathrm{C}_{17} \mathrm{H}_{17} \mathrm{NO}_{2} \mathrm{Na}[\mathrm{M}+\mathrm{Na}]^{+} 290.1152$, found 290.1153. Spectral and physical data are in accordance with literature. ${ }^{15}$

\section{3-Allyl-3-ethoxyindolin-2-one 2e}<smiles>C=CCC1(OCC)C(=O)Nc2ccccc21</smiles> 
General procedure A was followed with 1e $(69.3 \mathrm{mg}, 0.40 \mathrm{mmol})$ and ethanol $(2.5 \mathrm{~mL})$. Purification was performed with flash column chromatography over silica gel $\left(20 \mathrm{~g} \mathrm{SiO}_{2}\right.$, heptane/EtOAc, 100/0 to 50/50, gradient) to afford 2e as a light yellow solid $(26.6 \mathrm{mg}$, 0.122 mmol, 31\%). ${ }^{\mathbf{1}} \mathbf{H}$ NMR (400 MHz, CDCl $): \delta 8.96$ (brs, 1H), 7.32-7.24 (m, 2H), 7.08 (t, $J=7.4 \mathrm{~Hz}, 1 \mathrm{H}), 6.92(\mathrm{~d}, J=7.7 \mathrm{~Hz}, 1 \mathrm{H}), 5.66-5.45(\mathrm{~m}, 1 \mathrm{H}), 5.05-4.96(\mathrm{~m}, 2 \mathrm{H}), 3.28(\mathrm{dq}, J=$ 14.0, 7.0 Hz, 1H), 3.15 (dq, $J=14.0,7.0 \mathrm{~Hz}, 1 \mathrm{H}), 2.77$ (dd, $J=13.3,6.4 \mathrm{~Hz}, 1 \mathrm{H}), 2.64$ (dd, $J$ $=13.3,8.2 \mathrm{~Hz}, 1 \mathrm{H}), 1.15(\mathrm{t}, J=7.0 \mathrm{~Hz}, 3 \mathrm{H}) .{ }^{\mathbf{1 3}} \mathbf{C}\left\{{ }^{1} \mathbf{H}\right\} \mathbf{N M R}(\mathbf{1 0 0} \mathbf{M H z}, \mathbf{C D C l}): \delta 178.9(\mathrm{C}=\mathrm{O})$, $141.1(\mathrm{C}), 130.7(\mathrm{CH}), 129.8(\mathrm{CH}), 128.0(\mathrm{C}), 124.9(\mathrm{CH}), 123.0(\mathrm{CH}), 119.8\left(\mathrm{CH}_{2}\right), 110.5$ $(\mathrm{CH}), 82.6(\mathrm{C}), 61.2\left(\mathrm{CH}_{2}\right), 42.3\left(\mathrm{CH}_{2}\right), 15.5\left(\mathrm{CH}_{3}\right)$. FT-IR (neat, $\left.\mathbf{c m}^{-1}\right)$ : 3164, 2971, 2903 , 1730, 1697, 1618, 1603, 1469, 1231. HRMS (ESI $\left.{ }^{+}\right): m / z$ calcd. for $\mathrm{C}_{13} \mathrm{H}_{15} \mathrm{NO}_{2} \mathrm{Na}[\mathrm{M}+\mathrm{Na}]^{+}$ 240.0995 , found 240.0996 .

\section{3-Cyclopentyl-3-ethoxyindolin-2-one $2 \mathrm{f}$}

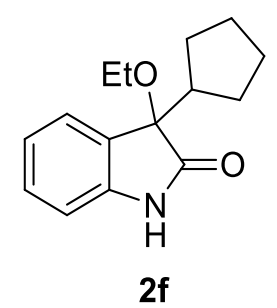

General procedure A was followed with 1 f $(80.5 \mathrm{mg}, 0.40 \mathrm{mmol})$ and ethanol $(2.5 \mathrm{~mL})$. Purification was performed with flash column chromatography over silica gel $\left(20 \mathrm{~g} \mathrm{SiO}_{2}\right.$, heptane/EtOAc, 100/0 to 50/50, gradient) to afford $2 \mathbf{f}$ as a light yellow solid (48.5 mg,

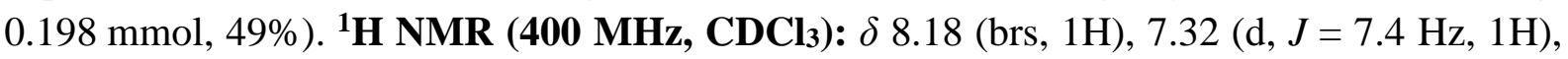
7.28-7.22 (m, 1H), $7.05(\mathrm{td}, J=7.6,0.9 \mathrm{~Hz}, 1 \mathrm{H}), 6.87(\mathrm{~d}, J=7.6 \mathrm{~Hz}, 1 \mathrm{H}), 3.26(\mathrm{dq}, J=14.0$, $7.0 \mathrm{~Hz}, 1 \mathrm{H}), 3.12(\mathrm{dq}, J=14.0,7.0 \mathrm{~Hz}, 1 \mathrm{H}), 2.55-2.41(\mathrm{~m}, 1 \mathrm{H}), 1.78-1.70(\mathrm{~m}, 1 \mathrm{H}), 1.62-1.56$ (m, 1H), 1.54-1.42 (m, 4H), 1.28-1.22 (m, 1H), $1.14(\mathrm{t}, J=7.0 \mathrm{~Hz}, 3 \mathrm{H}), 0.90-0.82(\mathrm{~m}, 1 \mathrm{H})$. ${ }^{13} \mathbf{C}\left\{{ }^{1} \mathbf{H}\right\}$ NMR (100 MHz, CDCl3): $\delta 179.1(\mathrm{C}=\mathrm{O}), 141.2(\mathrm{C}), 129.5(\mathrm{CH}), 128.2(\mathrm{C}), 125.3$ $(\mathrm{CH}), 122.7(\mathrm{CH}), 110.1(\mathrm{CH}), 84.8(\mathrm{C}), 61.4\left(\mathrm{CH}_{2}\right), 47.3(\mathrm{CH}), 26.6\left(\mathrm{CH}_{2}\right), 26.3\left(\mathrm{CH}_{2}\right), 25.4$ $\left(\mathrm{CH}_{2}\right), 25.3\left(\mathrm{CH}_{2}\right), 15.6\left(\mathrm{CH}_{3}\right)$. FT-IR (neat, $\left.\mathbf{c m}^{-1}\right)$ : 3149, 3090, 2950, 2874, 1725, 1684, 1618, 1599, 1467, 1212, 1115, 1089. HRMS (ESI $\left.{ }^{+}\right): m / z$ calcd. for $\mathrm{C}_{15} \mathrm{H}_{19} \mathrm{NO}_{2} \mathrm{Na}[\mathrm{M}+\mathrm{Na}]^{+}$ 268.1308 , found 268.1307 .

\section{3-Ethoxy-3-(pent-4-en-1-yl)indolin-2-one $2 \mathrm{~g}$}

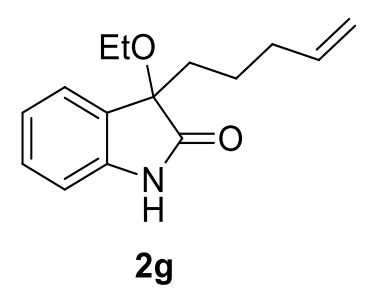

General procedure A was followed with $1 \mathrm{~g}(80.5 \mathrm{mg}, 0.40 \mathrm{mmol})$ and ethanol $(2.5 \mathrm{~mL})$. Purification was performed with flash column chromatography over silica gel $\left(20 \mathrm{~g} \mathrm{SiO}_{2}\right.$, heptane/EtOAc, 100/0 to 50/50, gradient) to afford $\mathbf{2 g}$ as a light yellow solid $(27.1 \mathrm{mg}$, 0.110 mmol, 28\%). ${ }^{1} \mathbf{H}$ NMR (400 MHz, CDCl 3 ): $\delta 7.92$ (brs, $\left.1 \mathrm{H}\right), 7.33-7.24$ (m, 2H), 7.08 (td, $J=7.7,1.1 \mathrm{~Hz}, 1 \mathrm{H}), 6.87(\mathrm{~d}, J=7.7 \mathrm{~Hz}, 1 \mathrm{H}), 5.68(\mathrm{ddt}, J=16.9,10.2,6.7 \mathrm{~Hz}, 1 \mathrm{H}), 5.02-4.81$ 
$(\mathrm{m}, 2 \mathrm{H}), 3.26(\mathrm{dq}, J=14.0,7.0 \mathrm{~Hz}, 1 \mathrm{H}), 3.12(\mathrm{dq}, J=14.0,7.0 \mathrm{~Hz}, 1 \mathrm{H}), 2.03-1.90(\mathrm{~m}, 4 \mathrm{H})$, 1.40-1.30 (m, 1H), 1.24-1.17 (m, 1H), $1.14(\mathrm{t}, J=7.0 \mathrm{~Hz}, 3 \mathrm{H}) .{ }^{\mathbf{1 3}} \mathbf{C}\left\{{ }^{1} \mathbf{H}\right\}$ NMR $(\mathbf{1 0 0} \mathbf{M H z}$, $\left.\mathbf{C D C l}_{3}\right): \delta 178.6(\mathrm{C}=\mathrm{O}), 140.9(\mathrm{C}), 138.2(\mathrm{CH}), 129.7(\mathrm{CH}), 128.7(\mathrm{C}), 124.7(\mathrm{CH}), 123.1(\mathrm{CH})$, $115.1\left(\mathrm{CH}_{2}\right), 110.2(\mathrm{CH}), 82.7(\mathrm{C}), 61.1\left(\mathrm{CH}_{2}\right), 37.6\left(\mathrm{CH}_{2}\right), 33.8\left(\mathrm{CH}_{2}\right), 22.2\left(\mathrm{CH}_{2}\right), 15.6\left(\mathrm{CH}_{3}\right)$. FT-IR (neat, $\mathbf{c m}^{-1}$ ): 3230, 2975, 2923, 1716, 1619, 1470, 1209. HRMS (ESI $\left.{ }^{+}\right): \mathrm{m} / z$ calcd. for $\mathrm{C}_{15} \mathrm{H}_{19} \mathrm{NO}_{2} \mathrm{Na}[\mathrm{M}+\mathrm{Na}]^{+} 268.1308$, found 268.1308 .

\section{3-Ethoxy-3-pentylindolin-2-one $2 \mathrm{~h}$}

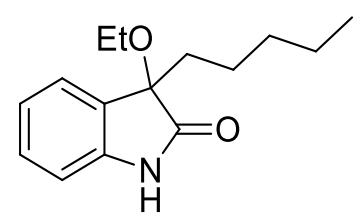

$2 h$

General procedure A was followed with $\mathbf{1 h}(81.3 \mathrm{mg}, 0.40 \mathrm{mmol})$ and ethanol $(2.5 \mathrm{~mL})$ Purification was performed with flash column chromatography over silica gel $\left(20 \mathrm{~g} \mathrm{SiO}_{2}\right.$, heptane/EtOAc, $100 / 0$ to 50/50, gradient) to afford $\mathbf{2 h}$ as a light brown solid (33.1 $\mathrm{mg}$, 0.134 mmol, 34\%). ${ }^{\mathbf{1}} \mathbf{H}$ NMR (400 MHz, CDCl $): \delta 8.08$ (brs, 1H), 7.30-7.24 (m, 2H), 7.09 (t, $J=7.5 \mathrm{~Hz}, 1 \mathrm{H}), 6.91-6.85(\mathrm{~m}, 1 \mathrm{H}), 3.26(\mathrm{dq}, J=14.0,7.0 \mathrm{~Hz}, 1 \mathrm{H}), 3.12(\mathrm{dq}, J=14.1,7.0 \mathrm{~Hz}$, $1 \mathrm{H}), 1.95(\mathrm{dd}, J=8.6,4.4 \mathrm{~Hz}, 2 \mathrm{H}), 1.64-1.60(\mathrm{~m}, 1 \mathrm{H}), 1.23-1.18(\mathrm{~m}, 4 \mathrm{H}), 1.14(\mathrm{t}, J=7.0 \mathrm{~Hz}$, 3H), 1.10-1.01 (m, 1H), $0.80(\mathrm{t}, J=6.6 \mathrm{~Hz}, 3 \mathrm{H}) .{ }^{13} \mathbf{C}\left\{{ }^{1} \mathbf{H}\right\}$ NMR (100 MHz, CDCl 3$): \delta 179.0$ $(\mathrm{C}=\mathrm{O}), 141.0(\mathrm{C}), 129.6(\mathrm{CH}), 128.8(\mathrm{C}), 124.6(\mathrm{CH}), 123.1(\mathrm{CH}), 110.2(\mathrm{CH}), 82.9(\mathrm{C}), 61.1$ $\left(\mathrm{CH}_{2}\right), 38.0\left(\mathrm{CH}_{2}\right), 31.9\left(\mathrm{CH}_{2}\right), 22.4\left(2 \mathrm{CH}_{2}\right), 15.6\left(\mathrm{CH}_{3}\right), 14.1\left(\mathrm{CH}_{3}\right)$. FT-IR (neat, cm $\left.{ }^{-1}\right)$ : 2955, 2926, 2871, 1716, 1618, 1469, 1211, 1188. HRMS (ESI $\left.{ }^{+}\right): m / z$ calcd. for $\mathrm{C}_{15} \mathrm{H}_{21} \mathrm{NO}_{2} \mathrm{Na}$ $[\mathrm{M}+\mathrm{Na}]^{+} 270.1465$, found 270.1463 .

\section{3-((1,3-dioxolan-2-yl)methyl)-3-ethoxyindolin-2-one $2 \mathrm{i}$}<smiles>CCOC1(CC2OCCO2)C(=O)Nc2ccccc21</smiles>

General procedure A was followed with $1 \mathbf{1 i}(87.7 \mathrm{mg}, 0.40 \mathrm{mmol})$ and ethanol $(2.5 \mathrm{~mL})$ Purification was performed with flash column chromatography over silica gel $\left(20 \mathrm{~g} \mathrm{SiO}_{2}\right.$, heptane/EtOAc, 100/0 to 50/50, gradient) to afford $\mathbf{2 i}$ as a white solid (26.0 $\mathrm{mg}, 0.10 \mathrm{mmol}$,

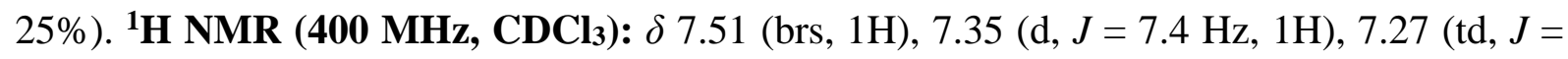
7.7, $1.1 \mathrm{~Hz}, 1 \mathrm{H}), 7.08(\mathrm{t}, J=7.5 \mathrm{~Hz}, 1 \mathrm{H}), 6.85(\mathrm{~d}, J=7.7 \mathrm{~Hz}, 1 \mathrm{H}), 4.78(\mathrm{dd}, J=6.0,4.0 \mathrm{~Hz}$, $1 \mathrm{H}), 3.86-3.80(\mathrm{~m}, 1 \mathrm{H}), 3.79-3.74(\mathrm{~m}, 1 \mathrm{H}), 3.73-3.67(\mathrm{~m}, 2 \mathrm{H}), 3.26(\mathrm{dq}, J=14.1,7.0 \mathrm{~Hz}, 1 \mathrm{H})$, $2.74(\mathrm{dq}, J=14.1,7.0 \mathrm{~Hz}, 1 \mathrm{H}), 2.43-2.40(\mathrm{~m}, 2 \mathrm{H}), 1.13(\mathrm{t}, J=7.0 \mathrm{~Hz}, 3 \mathrm{H}) .{ }^{13} \mathbf{C}\left\{{ }^{1} \mathbf{H}\right\} \mathbf{~ N M R}$ (100 MHz, CDCl$): ~ \delta 177.4(\mathrm{C}=\mathrm{O}), 141.2(\mathrm{C}), 130.0(\mathrm{CH}), 127.5(\mathrm{C}), 125.4(\mathrm{CH}), 123.0(\mathrm{CH})$, $110.1(\mathrm{CH}), 101.0(\mathrm{CH}), 79.7(\mathrm{C}), 64.8\left(\mathrm{CH}_{2}\right), 64.7\left(\mathrm{CH}_{2}\right), 60.3\left(\mathrm{CH}_{2}\right), 41.7\left(\mathrm{CH}_{2}\right), 15.3\left(\mathrm{CH}_{3}\right)$. 
FT-IR (neat, $\mathbf{c m}^{-1}$ ): 3263, 2974, 2927, 2887, 1725, 1619, 1471, 1193, 1107, 1085, 1048. HRMS (ESI $\left.{ }^{+}\right): m / z$ calcd. for $\mathrm{C}_{14} \mathrm{H}_{17} \mathrm{NO}_{4} \mathrm{Na}[\mathrm{M}+\mathrm{Na}]^{+} 286.1050$, found 286.1049.

\section{2-(3-Ethoxy-2-oxoindolin-3-yl)acetonitrile $2 \mathrm{j}$}<smiles>CCOC1(CC#N)C(=O)Nc2ccccc21</smiles>

2j

General procedure A was followed with $\mathbf{1 j}(68.9 \mathrm{mg}, 0.40 \mathrm{mmol})$ and ethanol $(2.5 \mathrm{~mL})$ Purification was performed with flash column chromatography over silica gel $\left(20 \mathrm{~g} \mathrm{SiO}_{2}\right.$, heptane/EtOAc, $100 / 0$ to $50 / 50$, gradient) to afford $\mathbf{2} \mathbf{j}$ as a light brown solid $(20.5 \mathrm{mg}$, 0.095 mmol, 24\%). ${ }^{1} \mathbf{H}$ NMR (400 MHz, CDCl $): \delta 8.60$ (brs, $\left.1 \mathrm{H}\right), 7.56$ (d, $\left.J=7.5 \mathrm{~Hz}, 1 \mathrm{H}\right)$, $7.37(\mathrm{td}, J=7.7,1.1 \mathrm{~Hz}, 1 \mathrm{H}), 7.17(\mathrm{td}, J=7.6,0.7 \mathrm{~Hz}, 1 \mathrm{H}), 6.96(\mathrm{dd}, J=7.7,2.2 \mathrm{~Hz}, 1 \mathrm{H})$, 3.34.3.23 (m, 1H), 3.23-3.15 (m, 1H), $3.06(\mathrm{~d}, J=16.4 \mathrm{~Hz}, 1 \mathrm{H}), 2.74(\mathrm{~d}, J=16.4 \mathrm{~Hz}, 1 \mathrm{H}), 1.17$

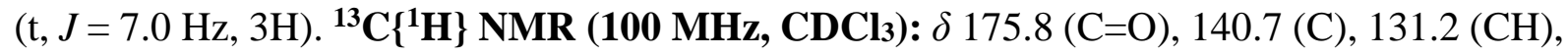
$125.3(\mathrm{C}), 125.2(\mathrm{CH}), 123.9(\mathrm{CH}), 115.5(\mathrm{C}), 111.1(\mathrm{CH}), 78.1(\mathrm{C}), 62.0\left(\mathrm{CH}_{2}\right), 27.0\left(\mathrm{CH}_{2}\right)$, $15.3\left(\mathrm{CH}_{3}\right)$. FT-IR (neat, $\left.\mathbf{c m}^{-1}\right):$ 3230, 2971, 2932, 1738, 1698, 1618, 1469, 1227, 1115, 1045. HRMS (ESI ${ }^{+}$): $m / z$ calcd. for $\mathrm{C}_{12} \mathrm{H}_{12} \mathrm{~N}_{2} \mathrm{O}_{2} \mathrm{Na}[\mathrm{M}+\mathrm{Na}]^{+} 239.0791$, found 239.0787 .

Ethyl 2-(3-ethoxy-2-oxoindolin-3-yl)acetate 2k<smiles>CCOCC1(OCC)C(=O)Nc2ccccc21</smiles>

General procedure A was followed with $1 \mathbf{k}(87.7 \mathrm{mg}, 0.40 \mathrm{mmol})$ and ethanol $(2.5 \mathrm{~mL})$ Purification was performed with flash column chromatography over silica gel $\left(20 \mathrm{~g} \mathrm{SiO}_{2}\right.$, heptane/EtOAc, 100/0 to 50/50, gradient) to afford $\mathbf{2 k}$ as a light yellow solid (36.0 mg, 0.137 mmol, 34\%). ${ }^{\mathbf{1}} \mathbf{H}$ NMR (400 MHz, CDCl $): \delta 8.80$ (brs, $\left.1 \mathrm{H}\right), 7.33$ (d, $\left.J=7.4 \mathrm{~Hz}, 1 \mathrm{H}\right)$, $7.27(\mathrm{td}, J=7.7,1.1 \mathrm{~Hz}, 1 \mathrm{H}), 7.06(\mathrm{td}, J=7.7,0.7 \mathrm{~Hz}, 1 \mathrm{H}), 6.90(\mathrm{~d}, J=7.8, \mathrm{~Hz}, 1 \mathrm{H}), 3.98-3.83$ $(\mathrm{m}, 2 \mathrm{H}), 3.29$ (dd, $J=8.4,7.0 \mathrm{~Hz}, 1 \mathrm{H}), 3.20(\mathrm{~d}, J=15.4 \mathrm{~Hz}, 1 \mathrm{H}), 3.14-3.03(\mathrm{~m}, 2 \mathrm{H}), 1.11(\mathrm{t}, J$ $=7.0 \mathrm{~Hz}, 3 \mathrm{H}), 1.01(\mathrm{t}, J=7.1 \mathrm{~Hz}, 3 \mathrm{H}) .{ }^{13} \mathbf{C}\left\{{ }^{1} \mathbf{H}\right\} \mathbf{N M R}\left(\mathbf{1 0 0} \mathbf{M H z}, \mathbf{C D C l}_{3}\right): \delta 177.6(\mathrm{C}=\mathrm{O})$, $168.8(\mathrm{C}=\mathrm{O}), 142.1(\mathrm{C}), 130.3(\mathrm{CH}), 126.9(\mathrm{C}), 124.8(\mathrm{CH}), 123.0(\mathrm{CH}), 110.5(\mathrm{CH}), 79.2(\mathrm{C})$, $60.8\left(\mathrm{CH}_{2}\right), 60.4\left(\mathrm{CH}_{2}\right), 42.1\left(\mathrm{CH}_{2}\right), 15.4\left(\mathrm{CH}_{3}\right), 13.9\left(\mathrm{CH}_{3}\right)$. FT-IR (neat, $\left.\mathbf{~ c m}^{-1}\right)$ : 3328, 3169 , 2976, 2928, 1736, 1714, 1618, 1470, 1371, 1342, 1188. HRMS (ESI $\left.{ }^{+}\right): ~ m / z$ calcd. for $\mathrm{C}_{14} \mathrm{H}_{17} \mathrm{NO}_{4} \mathrm{Na}[\mathrm{M}+\mathrm{Na}]^{+} 286.1050$, found 286.1044 . 


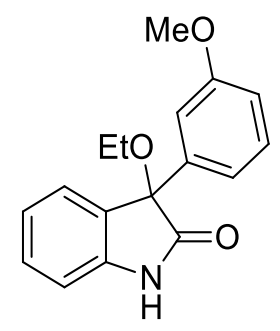

2l

General procedure A was followed with 11 (95.7 $\mathrm{mg}, 0.40 \mathrm{mmol})$ and ethanol $(2.5 \mathrm{~mL})$ Purification was performed with flash column chromatography over silica gel $\left(20 \mathrm{~g} \mathrm{SiO}_{2}\right.$, heptane/EtOAc, $100 / 0$ to $50 / 50$, gradient) to afford $2 \mathbf{l}$ as a light yellow solid $(28.0 \mathrm{mg}$, 0.099 mmol, 25\%). ${ }^{\mathbf{1}} \mathbf{H}$ NMR (400 MHz, CDCl3): $\delta 8.76$ (brs, $\left.1 \mathrm{H}\right), 7.30$ (t, $\left.J=7.7 \mathrm{~Hz}, 1 \mathrm{H}\right)$, 7.25-7.18 (m, 2H), 7.11-7.05 (m, 2H), 6.96-6.90 (m, 2H), $6.83(\mathrm{dd}, J=8.2,2.0 \mathrm{~Hz}, 1 \mathrm{H}), 3.78$ $(\mathrm{s}, 3 \mathrm{H}), 3.50(\mathrm{dq}, J=14.1,7.0 \mathrm{~Hz}, 1 \mathrm{H}), 3.34(\mathrm{dq}, J=14.1,7.0 \mathrm{~Hz}, 1 \mathrm{H}), 1.26(\mathrm{t}, J=7.0 \mathrm{~Hz}, 3 \mathrm{H})$. ${ }^{13} \mathbf{C}\left\{{ }^{1} \mathbf{H}\right\}$ NMR (100 MHz, CDCl $)$ : $\delta 178.1(\mathrm{C}=\mathrm{O}), 159.8(\mathrm{C}), 141.6(\mathrm{C}), 140.5(\mathrm{C}), 130.1$ $(\mathrm{CH}), 129.5(\mathrm{CH}), 129.3(\mathrm{C}), 126.0(\mathrm{CH}), 123.4(\mathrm{CH}), 118.6(\mathrm{CH}), 113.9(\mathrm{CH}), 112.3(\mathrm{CH})$, $110.7(\mathrm{CH}), 83.9(\mathrm{C}), 61.3\left(\mathrm{CH}_{2}\right), 55.4\left(\mathrm{CH}_{3}\right), 15.6\left(\mathrm{CH}_{3}\right)$. FT-IR (neat, $\left.\mathbf{c m}^{-1}\right): .3150,3091$, 2950, 2874, 1725, 1684, 1618, 1467, 1212, 1152. HRMS (ESI $\left.{ }^{+}\right): \mathrm{m} / z$ calcd. for $\mathrm{C}_{17} \mathrm{H}_{17} \mathrm{NO}_{3} \mathrm{Na}$ $[\mathrm{M}+\mathrm{Na}]^{+}$306.1101, found 306.1097.

\section{3-Ethoxy-3-(4-methoxyphenyl)indolin-2-one $2 \mathrm{~m}$}<smiles>CCOC1(c2ccc(OC)cc2)C(=O)Nc2ccccc21</smiles>

$2 \mathrm{~m}$

General procedure A was followed with $1 \mathrm{~m}(95.7 \mathrm{mg}, 0.40 \mathrm{mmol})$ and ethanol $(2.5 \mathrm{~mL})$ Purification was performed with flash column chromatography over silica gel $\left(20 \mathrm{~g} \mathrm{SiO}_{2}\right.$, heptane/EtOAc, 100/0 to 50/50, gradient) to afford $\mathbf{2 m}$ as a light yellow solid $(78.7 \mathrm{mg}$, 0.278 mmol, 69\%). ${ }^{\mathbf{1}} \mathbf{H}$ NMR (400 MHz, CDCl $): \delta 8.88$ (brs, $\left.1 \mathrm{H}\right), 7.34$ (d, $\left.J=8.9 \mathrm{~Hz}, 2 \mathrm{H}\right)$, $7.30(\mathrm{t}, J=7.8 \mathrm{~Hz}, 1 \mathrm{H}), 7.25(\mathrm{~d}, J=8.3 \mathrm{~Hz}, 1 \mathrm{H}), 7.10(\mathrm{t}, J=7.5 \mathrm{~Hz}, 1 \mathrm{H}), 6.94(\mathrm{~d}, J=7.8 \mathrm{~Hz}$, $1 \mathrm{H}), 6.85$ (d, $J=8.9 \mathrm{~Hz}, 2 \mathrm{H}), 3.77$ (s, 3H), 3.48 (dq, $J=14.0,7.0 \mathrm{~Hz}, 1 \mathrm{H}), 3.31$ (dq, $J=14.0$, $7.0 \mathrm{~Hz}, 1 \mathrm{H}), 1.25(\mathrm{t}, J=7.0 \mathrm{~Hz}, 3 \mathrm{H}) .{ }^{\mathbf{1}} \mathbf{C}\left\{{ }^{1} \mathbf{H}\right\} \mathbf{~ N M R}\left(\mathbf{1 0 0} \mathbf{M H z}, \mathbf{C D C l}_{3}\right): \delta 178.4(\mathrm{C}=\mathrm{O}), 159.8$ (C), $141.6(\mathrm{C}), 131.0(\mathrm{C}), 130.0(\mathrm{CH}), 129.2(\mathrm{C}), 127.8(2 \mathrm{CH}), 126.0(\mathrm{CH}), 123.3(\mathrm{CH}), 114.0$ $(2 \mathrm{CH}), 110.7(\mathrm{CH}), 83.6(\mathrm{C}), 61.2\left(\mathrm{CH}_{2}\right), 55.4\left(\mathrm{CH}_{3}\right), 15.6\left(\mathrm{CH}_{3}\right)$. FT-IR (neat, $\left.\mathbf{c m}^{-1}\right)$ : 3170 ,

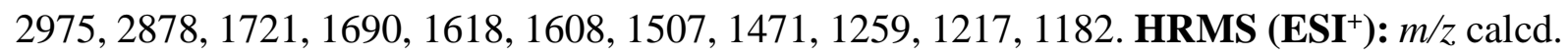
for $\mathrm{C}_{17} \mathrm{H}_{17} \mathrm{NO}_{3} \mathrm{Na}[\mathrm{M}+\mathrm{Na}]^{+}$306.1101, found 306.1091. 
<smiles>CCOC1(c2ccccc2)C(=O)Nc2ccccc21</smiles>

General procedure A was followed with 1n $(83.7 \mathrm{mg}, 0.40 \mathrm{mmol})$ and ethanol $(2.5 \mathrm{~mL})$ Purification was performed with flash column chromatography over silica gel $\left(20 \mathrm{~g} \mathrm{SiO}_{2}\right.$, heptane/EtOAc, 100/0 to 50/50, gradient) to afford $2 \mathrm{n}$ as a light yellow solid (51.0 mg, $0.201 \mathrm{mmol}, 50 \%)$. ${ }^{\mathbf{1}} \mathbf{H}$ NMR (600 MHz, CDCl3): $\delta 9.16$ (brs, 1H), 7.43-7.40 (m, 2H), 7.33$7.27(\mathrm{~m}, 4 \mathrm{H}), 7.23(\mathrm{~d}, J=7.4 \mathrm{~Hz}, 1 \mathrm{H}), 7.09(\mathrm{dd}, J=11.0,3.9 \mathrm{~Hz}, 1 \mathrm{H}), 6.96(\mathrm{~d}, J=7.8 \mathrm{~Hz}, 1 \mathrm{H})$, $3.50(\mathrm{dq}, J=14.0,7.0 \mathrm{~Hz}, 1 \mathrm{H}), 3.34(\mathrm{dq}, J=14.0,7.0 \mathrm{~Hz}, 1 \mathrm{H}), 1.26(\mathrm{t}, J=7.0 \mathrm{~Hz}, 3 \mathrm{H}) .{ }^{13} \mathbf{C}\left\{{ }^{1} \mathbf{H}\right\}$ NMR (150 MHz, CDCl $)$ ): $\delta 178.5$ (C=O), $141.7(\mathrm{C}), 139.0(\mathrm{C}), 130.1(\mathrm{CH}), 129.3$ (C), 128.6 $(2 \mathrm{CH}), 128.5(\mathrm{CH}), 126.3(2 \mathrm{CH}), 126.0(\mathrm{CH}), 123.4(\mathrm{CH}), 110.8(\mathrm{CH}), 84.0(\mathrm{C}), 61.2\left(\mathrm{CH}_{2}\right)$, $15.5\left(\mathrm{CH}_{3}\right)$. HRMS (ESI $\left.{ }^{+}\right): m / z$ calcd. for $\mathrm{C}_{16} \mathrm{H}_{15} \mathrm{NO}_{2} \mathrm{Na}[\mathrm{M}+\mathrm{Na}]^{+} 276.0995$, found 276.0993. Spectral and physical data are in accordance with literature. ${ }^{16}$

\section{3-Ethoxy-3-(p-tolyl)indolin-2-one 20}<smiles>CCOC1(c2ccc(C)cc2)C(=O)Nc2ccccc21</smiles>

20

General procedure A was followed with $10(89.3 \mathrm{mg}, 0.40 \mathrm{mmol})$ and ethanol $(2.5 \mathrm{~mL})$ Purification was performed with flash column chromatography over silica gel $\left(20 \mathrm{~g} \mathrm{SiO}_{2}\right.$, heptane/EtOAc, 100/0 to 50/50, gradient) to afford 20 as a light yellow solid (43.7 mg, 0.163 mmol, 41\%). ${ }^{1} \mathbf{H}$ NMR (400 MHz, CDCl3): $\delta 9.24$ (brs, $\left.1 \mathrm{H}\right), 7.33-7.27$ (m, 3H), 7.23 (d, $J=7.4 \mathrm{~Hz}, 1 \mathrm{H}), 7.13(\mathrm{~d}, J=8.1 \mathrm{~Hz}, 2 \mathrm{H}), 7.08(\mathrm{t}, J=7.4 \mathrm{~Hz}, 1 \mathrm{H}), 6.94(\mathrm{~d}, J=7.8 \mathrm{~Hz}, 1 \mathrm{H}), 3.49$ (dq, $J=14.1,7.0 \mathrm{~Hz}, 1 \mathrm{H}), 3.33(\mathrm{dq}, J=14.1,7.0 \mathrm{~Hz}, 1 \mathrm{H}), 2.31(\mathrm{~s}, 3 \mathrm{H}), 1.26(\mathrm{t}, J=7.0 \mathrm{~Hz}, 3 \mathrm{H})$. ${ }^{13} \mathbf{C}\left\{{ }^{1} \mathbf{H}\right\}$ NMR (100 MHz, CDCl3): $\delta 178.7$ (C=O), 141.7 (C), 138.3 (C), 136.0 (C), 130.0 $(\mathrm{CH}), 129.4(\mathrm{C}), 129.3(2 \mathrm{CH}), 126.3(2 \mathrm{CH}), 125.9(\mathrm{CH}), 129.3(\mathrm{CH}), 110.8(\mathrm{CH}), 83.9(\mathrm{C})$, $61.2\left(\mathrm{CH}_{2}\right), 21.3\left(\mathrm{CH}_{3}\right), 15.6\left(\mathrm{CH}_{3}\right)$. FT-IR (neat, $\left.\mathbf{c m}^{-1}\right)$ : 3231, 2975, 1714, 1616, 1469, 1207 , 1175. HRMS (ESI $\left.{ }^{+}\right): m / z$ calcd. for $\mathrm{C}_{17} \mathrm{H}_{17} \mathrm{NO}_{2} \mathrm{Na}[\mathrm{M}+\mathrm{Na}]^{+} 290.1152$, found 290.1152 . 


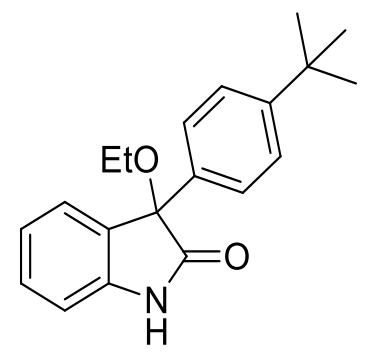

$2 p$

General procedure A was followed with 1p (106.0 mg, $0.40 \mathrm{mmol})$ and ethanol $(2.5 \mathrm{~mL})$ Purification was performed with flash column chromatography over silica gel $\left(20 \mathrm{~g} \mathrm{SiO}_{2}\right.$, heptane/EtOAc, 100/0 to 50/50, gradient) to afford $\mathbf{2 p}$ as a light yellow solid (46.7 $\mathrm{mg}$, 0.151 mmol, 38\%). ${ }^{1} \mathbf{H}$ NMR (400 MHz, CDCl $): \delta 9.10$ (brs, 1H), 7.37-7.32 (m, 4H), 7.31$7.27(\mathrm{~m}, 1 \mathrm{H}), 7.27-7.24(\mathrm{~m}, 1 \mathrm{H}), 7.09$ (t, $J=7.5 \mathrm{~Hz}, 1 \mathrm{H}), 6.95(\mathrm{~d}, J=7.8 \mathrm{~Hz}, 1 \mathrm{H}), 3.50$ (dq, $J$ $=14.1,7.0 \mathrm{~Hz}, 1 \mathrm{H}), 3.34(\mathrm{dq}, J=14.1,7.0 \mathrm{~Hz}, 1 \mathrm{H}), 1.28(\mathrm{~s}, 9 \mathrm{H}), 1.25(\mathrm{t}, J=7.0 \mathrm{~Hz}, 3 \mathrm{H})$. ${ }^{13} \mathbf{C}\left\{{ }^{1} \mathbf{H}\right\}$ NMR (100 MHz, CDCl3): $\delta 178.6(\mathrm{C}=\mathrm{O}), 151.4$ (C), 141.7 (C), 135.9 (C), 130.0 $(\mathrm{CH}), 129.3(\mathrm{C}), 126.1(2 \mathrm{CH}), 126.1(\mathrm{CH}), 125.3(2 \mathrm{CH}), 123.3(\mathrm{CH}), 110.8(\mathrm{CH}), 83.9(\mathrm{C})$, $61.2\left(\mathrm{CH}_{2}\right), 34.6(\mathrm{C}), 31.4\left(3 \mathrm{CH}_{3}\right), 15.6\left(\mathrm{CH}_{3}\right)$. FT-IR (neat, $\left.\mathbf{c m}^{-1}\right)$ : 2960, 1719, 1618, 1469 , 1218, 1193. HRMS (ESI $\left.{ }^{+}\right): m / z$ calcd. for $\mathrm{C}_{20} \mathrm{H}_{23} \mathrm{NO}_{2} \mathrm{Na}[\mathrm{M}+\mathrm{Na}]^{+} 332.1621$, found 332.1622 .

3-([1,1'-Biphenyl]-4-yl)-3-ethoxyindolin-2-one $2 \mathrm{q}$<smiles>CCOC1(c2ccc(-c3ccccc3)cc2)C(=O)Nc2ccccc21</smiles>

General procedure A was followed with 1q (114.0 mg, $0.40 \mathrm{mmol})$, ethanol (2.5 mL) in THF as solvent $(2.5 \mathrm{~mL})$ Purification was performed with flash column chromatography over silica gel (20 $\mathrm{g} \mathrm{SiO}_{2}$, heptane/EtOAc, 100/0 to 50/50, gradient) to afford $\mathbf{2 q}$ as a light yellow solid (60.0 mg, $0.182 \mathrm{mmol}, 46 \%)$. ${ }^{\mathbf{1}} \mathbf{H}$ NMR (400 MHz, CDCl3) $\delta 7.99$ (s, 1H), 7.54 (m, 4H), 7.48 $(\mathrm{d}, J=8.4 \mathrm{~Hz}, 2 \mathrm{H}), 7.41(\mathrm{t}, J=7.6 \mathrm{~Hz}, 2 \mathrm{H}), 7.36-7.28(\mathrm{~m}, 3 \mathrm{H}), 7.13(\mathrm{td}, J=7.5,0.8 \mathrm{~Hz}, 1 \mathrm{H})$, $6.96(\mathrm{~d}, J=7.8 \mathrm{~Hz}, 1 \mathrm{H}), 3.53(\mathrm{dq}, J=14.0,7.0 \mathrm{~Hz}, 1 \mathrm{H}), 3.37(\mathrm{dq}, J=14.0,7.0 \mathrm{~Hz}, 1 \mathrm{H}), 1.28$ $(\mathrm{t}, J=7.0 \mathrm{~Hz}, 3 \mathrm{H}) .{ }^{13} \mathbf{C}\left\{{ }^{1} \mathbf{H}\right\}$ NMR (100 MHz, CDCl$) \boldsymbol{\delta} 177.4(\mathrm{C}=\mathrm{O}), 141.5(\mathrm{C}), 141.4(\mathrm{C})$, $140.9(\mathrm{C}), 138.0(\mathrm{C}), 130.2(\mathrm{CH}), 129.2(\mathrm{C}), 128.9(2 \mathrm{CH}), 127.5(\mathrm{CH}), 127.4(2 \mathrm{CH}), 127.3$ $(2 \mathrm{CH}), 126.9(2 \mathrm{CH}), 126.2(\mathrm{CH}), 123.5(\mathrm{CH}), 110.5(\mathrm{CH}), 83.7(\mathrm{C}), 61.3\left(\mathrm{CH}_{2}\right), 15.6\left(\mathrm{CH}_{3}\right)$. FT-IR (neat, $\mathbf{c m}^{-1}$ ): 3229, 2923, 2852, 1717, 1617, 1469, 1177, 1105. HRMS (ESI'): $m / z$ calcd. for $\mathrm{C}_{22} \mathrm{H}_{19} \mathrm{NO}_{2} \mathrm{Na}[\mathrm{M}+\mathrm{Na}]^{+} 352.1308$, found 352.1289 . 


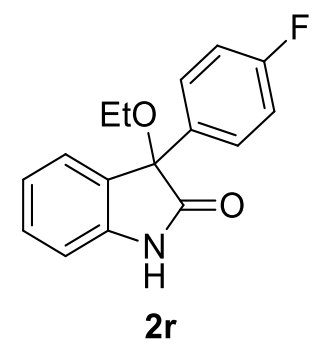

General procedure A was followed with 1r (90.9 mg, $0.40 \mathrm{mmol})$ and ethanol $(2.5 \mathrm{~mL})$ Purification was performed with flash column chromatography over silica gel $\left(20 \mathrm{~g} \mathrm{SiO}_{2}\right.$, heptane/EtOAc, 100/0 to 50/50, gradient) to afford $\mathbf{2 r}$ as a light yellow solid (77 mg,

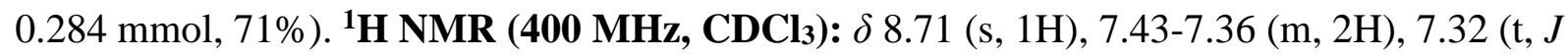
$=7.7 \mathrm{~Hz}, 1 \mathrm{H}), 7.22(\mathrm{~d}, J=7.4 \mathrm{~Hz}, 1 \mathrm{H}), 7.11(\mathrm{t}, J=7.5 \mathrm{~Hz}, 1 \mathrm{H}), 7.00(\mathrm{t}, J=8.6 \mathrm{~Hz}, 2 \mathrm{H}), 6.95$ $(\mathrm{d}, J=7.9 \mathrm{~Hz}, 1 \mathrm{H}), 3.47(\mathrm{dq}, J=14.0,7.0 \mathrm{~Hz}, 1 \mathrm{H}), 3.31(\mathrm{dq}, J=14.0,7.0 \mathrm{~Hz}, 1 \mathrm{H}), 1.25(\mathrm{t}, J=$ $6.9 \mathrm{~Hz}, 3 \mathrm{H}) .{ }^{13} \mathbf{C}\left\{{ }^{1} \mathbf{H}\right\}$ NMR (100 MHz, CDCl$): \delta 178.0(\mathrm{C}=\mathrm{O}), 162.9\left(\mathrm{~d}, J^{\mathrm{CF}}=247.1 \mathrm{~Hz}, \mathrm{C}\right)$, $141.6(\mathrm{C}), 134.8\left(\mathrm{~d}, J^{\mathrm{CF}}=247.1 \mathrm{~Hz}, \mathrm{C}\right), 130.3(\mathrm{CH}), 128.9(\mathrm{C}), 128.4(\mathrm{CH}), 128.3(\mathrm{CH}), 126.0$ $(\mathrm{CH}), 123.6(\mathrm{CH}), 115.4\left(\mathrm{~d}, J^{\mathrm{CF}}=21.4 \mathrm{~Hz}, 2 \mathrm{CH}\right), 110.8(\mathrm{CH}), 83.5(\mathrm{C}), 61.3\left(\mathrm{CH}_{2}\right), 15.5\left(\mathrm{CH}_{3}\right)$. ${ }^{19}$ F NMR (376 MHz, CDCl3): $\delta$-113.83. FT-IR (neat, $\mathbf{c m}^{-1}$ ): 3375, 3282, 2975, 2930, 2882, 1720, 1652, 1560, 1437, 1245, 1221. HRMS (ESI $\left.{ }^{+}\right): m / z$ calcd. for $\mathrm{C}_{16} \mathrm{H}_{14} \mathrm{FNO}_{2} \mathrm{Na}[\mathrm{M}+\mathrm{Na}]^{+}$ 294.0901, found 294.0902.

\section{3-(4-Chlorophenyl)-3-ethoxyindolin-2-one $2 \mathrm{~s}$}<smiles>CCOC1(c2ccc(Cl)cc2)C(=O)Nc2ccccc21</smiles>

2s

General procedure A was followed with $1 \mathrm{~s}(97.5 \mathrm{mg}, 0.40 \mathrm{mmol})$ and ethanol $(2.5 \mathrm{~mL})$. Purification was performed with flash column chromatography over silica gel $\left(20 \mathrm{~g} \mathrm{SiO}_{2}\right.$, heptane/EtOAc, $100 / 0$ to 50/50, gradient) to afford $2 \mathrm{~s}$ as a light yellow solid $(59.8 \mathrm{mg}$, 0.208 mmol, 52\%). ${ }^{\mathbf{1}} \mathbf{H}$ NMR (600 MHz, $\left.\mathbf{C D C l}_{3}\right): \delta 8.36$ (s, 1H), 7.35-7.31 (m, 2H), 7.29-7.22 $(\mathrm{m}, 2 \mathrm{H}), 7.19(\mathrm{~s}, 1 \mathrm{H}), 7.12-7.05(\mathrm{~m}, 1 \mathrm{H}), 6.94(\mathrm{~d}, J=7.8 \mathrm{~Hz}, 1 \mathrm{H}), 3.53-3.39(\mathrm{~m}, 1 \mathrm{H}), 3.34-3.25$ $(\mathrm{m}, 1 \mathrm{H}), 1.24(\mathrm{t}, J=7.0 \mathrm{~Hz}, 3 \mathrm{H}) .{ }^{\mathbf{1 3}} \mathbf{C}\left\{{ }^{\mathbf{1}} \mathbf{H}\right\} \mathbf{~ N M R}\left(\mathbf{1 5 0} \mathbf{M H z}, \mathbf{C D C l}_{3}\right) \delta 177.4(\mathrm{C}=\mathrm{O}), 141.5(\mathrm{C})$, $137.6(\mathrm{C}), 134.5(\mathrm{C}), 130.4(\mathrm{CH}), 128.8(\mathrm{C}), 128.7(2 \mathrm{CH}), 127.9(2 \mathrm{CH}), 126.0(\mathrm{CH}), 123.6$ $(\mathrm{CH}), 110.7(\mathrm{CH}), 83.4(\mathrm{C}), 61.4\left(\mathrm{CH}_{2}\right), 15.5\left(\mathrm{CH}_{3}\right)$. FT-IR (neat, $\left.\mathbf{c m}^{-1}\right)$ : 3208, 1715, 1617, 1487, 1469, 1210, 1175. HRMS (ESI $\left.{ }^{+}\right): m / z$ calcd. for $\mathrm{C}_{16} \mathrm{H}_{14} \mathrm{ClNO}_{2} \mathrm{Na}[\mathrm{M}+\mathrm{Na}]^{+} 310.0606$, found 310.0605 . 
<smiles>CCOC1(c2ccc(OC)cc2)C(=O)Nc2c(Cl)cccc21</smiles>

General procedure A was followed with 3a $(109 \mathrm{mg}, 0.40 \mathrm{mmol})$ and ethanol $(2.5 \mathrm{~mL})$. Purification was performed with flash column chromatography over silica gel $\left(20 \mathrm{~g} \mathrm{SiO}_{2}\right.$, heptane/EtOAc, 100/0 to 50/50, gradient) to afford $\mathbf{4 a}$ as a colourless oil (32.0 mg, $0.101 \mathrm{mmol}$,

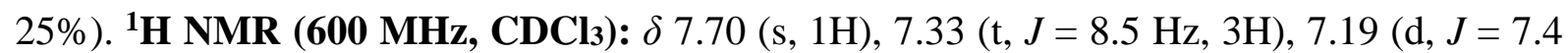
$\mathrm{Hz}, 1 \mathrm{H}), 7.07$ (t, $J=7.8 \mathrm{~Hz}, 1 \mathrm{H}), 6.85$ (d, $J=8.8 \mathrm{~Hz}, 2 \mathrm{H}), 3.78$ (s, 3H), 3.48 (dq, $J=14.1,6.9$ $\mathrm{Hz}, 1 \mathrm{H}), 3.32(\mathrm{dq}, J=14.1,6.9 \mathrm{~Hz}, 1 \mathrm{H}), 1.25(\mathrm{t}, J=6.9 \mathrm{~Hz}, 3 \mathrm{H}) .{ }^{13} \mathbf{C}\left\{{ }^{1} \mathbf{H}\right\}$ NMR $(\mathbf{1 5 0} \mathbf{~ M H z}$, CDCl3): $\delta 176.3(\mathrm{C}=\mathrm{O}), 160.1(\mathrm{C}), 139.0(\mathrm{C}), 130.7(\mathrm{C}), 130.3(\mathrm{C}), 129.90(\mathrm{CH}), 127.8(2 \mathrm{CH})$, 124.4 (CH), $124.2(\mathrm{CH}), 115.7(\mathrm{C}), 114.1(2 \mathrm{CH}), 84.2(\mathrm{C}), 61.5\left(\mathrm{CH}_{2}\right), 55.4\left(\mathrm{CH}_{3}\right), 15.5\left(\mathrm{CH}_{3}\right)$. FT-IR (neat, $\mathbf{c m}^{-1}$ ): 3188, 2976, 2925, 1719, 1615, 1608, 1509, 1453, 1252, 1171. HRMS (ESI ${ }^{+}$): $m / z$ calcd. for $\mathrm{C}_{17} \mathrm{H}_{16} \mathrm{ClNO}_{3} \mathrm{Na}[\mathrm{M}+\mathrm{Na}]^{+} 340.0711$, found 340.0713 .

\section{3-Ethoxy-3-(4-methoxyphenyl)-7-(trifluoromethyl)indolin-2-one 4b}<smiles>CCOC1(c2ccc(OC)cc2)C(=O)Nc2c(C(F)(F)F)cccc21</smiles>

General procedure A was followed with $3 \mathbf{b}(123 \mathrm{mg}, 0.40 \mathrm{mmol})$ and ethanol $(2.5 \mathrm{~mL})$. Purification was performed with flash column chromatography over silica gel $\left(20 \mathrm{~g} \mathrm{SiO}_{2}\right.$, heptane/EtOAc, 100/0 to 50/50, gradient) to afford $\mathbf{4 b}$ as a white solid (46.1 $\mathrm{mg}, 0.311 \mathrm{mmol}$, 33\%). ${ }^{1} \mathbf{H}$ NMR (600 MHz, CDCl3): $\delta 7.67$ (brs, $\left.1 \mathrm{H}\right), 7.54$ (d, $\left.J=8.0 \mathrm{~Hz}, 1 \mathrm{H}\right), 7.46$ (d, $J=7.4$ $\mathrm{Hz}, 1 \mathrm{H}), 7.32$ (d, $J=8.9 \mathrm{~Hz}, 2 \mathrm{H}), 7.22$ (t, $J=7.7 \mathrm{~Hz}, 1 \mathrm{H}), 6.86(\mathrm{~d}, J=8.9 \mathrm{~Hz}, 2 \mathrm{H}), 3.78$ (s, $3 \mathrm{H}), 3.49(\mathrm{dq}, J=14.1,7.0 \mathrm{~Hz}, 1 \mathrm{H}), 3.31(\mathrm{dq}, J=14.1,7.0 \mathrm{~Hz}, 1 \mathrm{H}), 1.25(\mathrm{t}, J=6.9 \mathrm{~Hz}, 3 \mathrm{H})$. ${ }^{13} \mathbf{C}\left\{{ }^{1} \mathbf{H}\right\}$ NMR (150 MHz, CDCl$): ~ \delta 176.7(\mathrm{C}=\mathrm{O}), 160.1(\mathrm{C}), 138.8(\mathrm{C}), 131.0(\mathrm{C}), 130.0(\mathrm{C})$, $129.5(\mathrm{CH}), 127.8(2 \mathrm{CH}), 126.8\left(\mathrm{q}, J^{\mathrm{CF}}=4.0 \mathrm{~Hz}, \mathrm{CH}\right), 123.9\left(\mathrm{q}, J^{\mathrm{CF}}=270.0 \mathrm{~Hz}, \mathrm{C}\right), 123.1$ $(\mathrm{CH}), 114.1(2 \mathrm{CH}), 112.7\left(\mathrm{q}, J^{\mathrm{CF}}=33.3 \mathrm{~Hz}, \mathrm{C}\right), 82.2(\mathrm{C}), 61.5\left(\mathrm{CH}_{2}\right), 55.4\left(\mathrm{CH}_{3}\right), 15.5\left(\mathrm{CH}_{3}\right)$. ${ }^{19}$ F NMR (565 MHz, CDCl3): $\delta$-60.53. FT-IR (neat, cm$^{-1}$ ): 3229, 3124, 2975, 1734, 1611, 1509, 1452, 1342, 1255. HRMS (ESI $\left.{ }^{+}\right): m / z$ calcd. for $\mathrm{C}_{18} \mathrm{H}_{16} \mathrm{~F}_{3} \mathrm{NO}_{3} \mathrm{Na}[\mathrm{M}+\mathrm{Na}]^{+} 374.0975$, found 374.0976 . 
<smiles>CCOC1(c2ccc(OC)cc2)C(=O)Nc2cc(OC)ccc21</smiles>

General procedure A was followed with 3c (108 $\mathrm{mg}, 0.40 \mathrm{mmol})$ and ethanol $(2.5 \mathrm{~mL})$. Purification was performed with flash column chromatography over silica gel $\left(20 \mathrm{~g} \mathrm{SiO}_{2}\right.$, heptane/EtOAc, 100/0 to 50/50, gradient) to afford $\mathbf{4 c}$ as a yellow solid (73.0 mg, $0.233 \mathrm{mmol}$, 58\%). ${ }^{1} \mathbf{H}$ NMR (400 MHz, CDCl $): \delta 9.10$ (brs, $\left.1 \mathrm{H}\right), 7.34$ (d, $\left.J=8.9 \mathrm{~Hz}, 2 \mathrm{H}\right), 7.13$ (d, $J=8.2$ $\mathrm{Hz}, 1 \mathrm{H}), 6.84(\mathrm{~d}, J=8.9 \mathrm{~Hz}, 2 \mathrm{H}), 6.61(\mathrm{dd}, J=8.2,2.2 \mathrm{~Hz}, 1 \mathrm{H}), 6.54(\mathrm{~d}, \mathrm{~J}=2.2 \mathrm{~Hz}, 1 \mathrm{H}), 3.82$ (s, 3H), 3.77 (s, 3H), $3.45(\mathrm{dq}, J=14.1,7.0 \mathrm{~Hz}, 1 \mathrm{H}), 3.32((\mathrm{dq}, J=14.1,7.0 \mathrm{~Hz}, 1 \mathrm{H}), 1.24$ (t,

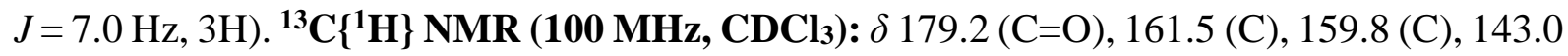
(C), $131.3(\mathrm{C}), 127.9(2 \mathrm{CH}), 126.9(\mathrm{CH}), 120.7(\mathrm{C}), 113.9(2 \mathrm{CH}), 108.4(\mathrm{CH}), 97.7(\mathrm{CH}), 83,4$ (C), $61.0\left(\mathrm{CH}_{2}\right), 55.7\left(\mathrm{CH}_{3}\right), 55.4\left(\mathrm{CH}_{3}\right), 15.6\left(\mathrm{CH}_{3}\right)$. FT-IR (neat, $\left.\mathbf{c m}^{-1}\right)$ : 3217, 2959, 2932, 2836, 1717, 1622, 1601, 1506, 1459, 1303, 1249, 1171. HRMS (ESI $\left.{ }^{+}\right): m / z$ calcd. for $\mathrm{C}_{18} \mathrm{H}_{19} \mathrm{NO}_{4} \mathrm{Na}[\mathrm{M}+\mathrm{Na}]^{+} 336.1207$, found 336.1204.

\section{3-Ethoxy-5-methoxy-3-(4-methoxyphenyl)indolin-2-one 4d}<smiles>CCOC1(c2ccc(OC)cc2)C(=O)Nc2ccc(OC)cc21</smiles>

General procedure A was followed with $\mathbf{3 d}(108 \mathrm{mg}, 0.40 \mathrm{mmol})$ and ethanol $(2.5 \mathrm{~mL})$. Purification was performed with flash column chromatography over silica gel $\left(20 \mathrm{~g} \mathrm{SiO}_{2}\right.$, heptane/EtOAc, 100/0 to 50/50, gradient) to afford $\mathbf{4 d}$ as a brown oil $(60.0 \mathrm{mg}, 0.19 \mathrm{mmol}$, 48\%). ${ }^{1} \mathbf{H}$ NMR (600 MHz, CDCl $): \delta 8.53$ (s, 1H), 7.34 (d, $\left.J=8.8 \mathrm{~Hz}, 2 \mathrm{H}\right), 6.86-6.25$ (m, $5 \mathrm{H}), 3.77$ (s, 3H), $3.77(\mathrm{~s}, 3 \mathrm{H}), 3.48(\mathrm{dq}, J=14.0,7.0 \mathrm{~Hz}, 1 \mathrm{H}), 3.32(\mathrm{dq}, J=14.0,7.0 \mathrm{~Hz}, 1 \mathrm{H})$, $1.25(\mathrm{t}, J=7.0 \mathrm{~Hz}, 3 \mathrm{H}) .{ }^{\mathbf{1 3}} \mathbf{C}\left\{{ }^{1} \mathbf{H}\right\} \mathbf{N M R}\left(\mathbf{1 5 0} \mathbf{~ M H z}, \mathbf{C D C l}_{3}\right): \delta 178.2(\mathrm{C}=\mathrm{O}), 159.8(\mathrm{C}), 156.5$ (C), $134.8(\mathrm{C}), 131.0(\mathrm{C}), 130.6(\mathrm{C}), 127.8(2 \mathrm{CH}), 115.0(\mathrm{CH}), 114.0(2 \mathrm{CH}), 112.4(\mathrm{CH}), 111.1$ $(\mathrm{CH}), 84.0(\mathrm{C}), 61.3\left(\mathrm{CH}_{2}\right), 55.9\left(\mathrm{CH}_{3}\right), 55.4\left(\mathrm{CH}_{3}\right), 15.6\left(\mathrm{CH}_{3}\right)$. FT-IR (neat, cm-1): 3238, 2975, 2932, 2836, 1713, 1607, 1509, 1485, 1438, 1297, 1249, 1275. HRMS (ESI ${ }^{+}$): $\mathrm{m} / \mathrm{z}$ calcd. for $\mathrm{C}_{18} \mathrm{H}_{19} \mathrm{NO}_{4} \mathrm{Na}[\mathrm{M}+\mathrm{Na}]^{+} 336.1207$, found 336.1204. 


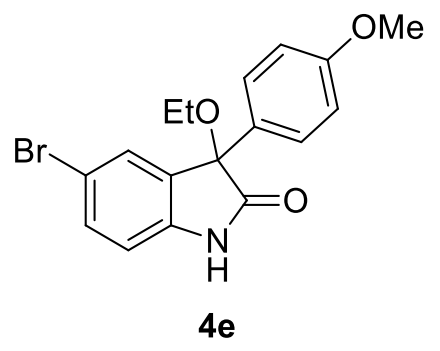

General procedure A was followed with 3 e $(127 \mathrm{mg}, 0.40 \mathrm{mmol})$ and ethanol $(2.5 \mathrm{~mL})$. Purification was performed with flash column chromatography over silica gel $\left(20 \mathrm{~g} \mathrm{SiO}_{2}\right.$, heptane/EtOAc, 100/0 to 50/50, gradient) to afford $4 \mathbf{e}$ as a light yellow solid (114 mg,

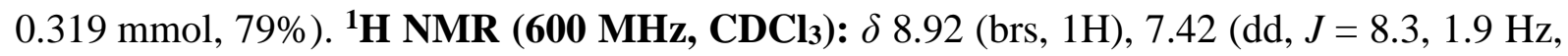
$1 \mathrm{H}), 7.35(\mathrm{~d}, J=1.8 \mathrm{~Hz}, 1 \mathrm{H}), 7.31(\mathrm{~d}, J=8.9 \mathrm{~Hz}, 2 \mathrm{H}), 6.86(\mathrm{~d}, J=8.9 \mathrm{~Hz}, 2 \mathrm{H}), 6.83(\mathrm{~d}, J=$ $8.3 \mathrm{~Hz}, 1 \mathrm{H}), 3.78(\mathrm{~s}, 3 \mathrm{H}), 3.47(\mathrm{dq}, J=14.0,7.0 \mathrm{~Hz}, 1 \mathrm{H}), 3.32(\mathrm{dq}, J=14.0,7.0 \mathrm{~Hz}, 1 \mathrm{H}), 1.26$ $(\mathrm{t}, J=6.9 \mathrm{~Hz}, 3 \mathrm{H}) .{ }^{13} \mathbf{C}\left\{{ }^{1} \mathbf{H}\right\}$ NMR (150 MHz, CDCl 3$): \delta 178.0(\mathrm{C}=\mathrm{O}), 160.0(\mathrm{C}), 140.5(\mathrm{C})$, $133.0(\mathrm{CH}), 131.6(\mathrm{C}), 130.3(\mathrm{C}), 129.0(\mathrm{CH}), 127.6(2 \mathrm{CH}), 116.1(\mathrm{C}), 114.1(2 \mathrm{CH}), 112.3$ $(\mathrm{CH}), 83.6(\mathrm{C}), 61.4\left(\mathrm{CH}_{2}\right), 55.4\left(\mathrm{CH}_{3}\right), 15.5\left(\mathrm{CH}_{3}\right)$. FT-IR (neat, $\left.\mathbf{c m}^{-1}\right)$ : 3215, 2974, 2929 , 2836, 1713, 1609, 1508, 1468, 1438, 1248, 1171. HRMS (ESI $\left.{ }^{+}\right): ~ m / z$ calcd. for $\mathrm{C}_{17} \mathrm{H}_{16} \mathrm{BrNO}_{3} \mathrm{Na}[\mathrm{M}+\mathrm{Na}]^{+}$384.0206, found 384.0203.

\section{3-Ethoxy-3-(4-methoxyphenyl)-5-nitroindolin-2-one $4 f$}<smiles>[Li]c1ccc([N+](=O)[O-])cc1C1(OCC)C(=O)Nc2ccc(OC)cc21</smiles>

General procedure A was followed with $3 \mathbf{f}(114 \mathrm{mg}, 0.40 \mathrm{mmol})$ and ethanol $(2.5 \mathrm{~mL})$. Purification was performed with flash column chromatography over silica gel $\left(20 \mathrm{~g} \mathrm{SiO}_{2}\right.$, heptane/EtOAc, 100/0 to 50/50, gradient) to afford $\mathbf{4 f}$ as a white solid (49.8 $\mathrm{mg}, 0.152 \mathrm{mmol}$, 38\%). ${ }^{1} \mathbf{H}$ NMR (500 MHz, CDCl $): \delta 8.55$ (s, 1H), 8.29 (dd, $\left.J=8.6,2.3 \mathrm{~Hz}, 1 \mathrm{H}\right), 8.17$ (d, $J=$ $2.3 \mathrm{~Hz}, 1 \mathrm{H}), 7.32(\mathrm{~d}, J=8.9 \mathrm{~Hz}, 2 \mathrm{H}), 7.05(\mathrm{~d}, J=8.6 \mathrm{~Hz}, 1 \mathrm{H}), 6.88(\mathrm{~d}, J=8.9 \mathrm{~Hz}, 2 \mathrm{H}), 3.79$ $(\mathrm{s}, 3 \mathrm{H}), 3.50(\mathrm{dq}, J=13.9,6.9 \mathrm{~Hz}, 1 \mathrm{H}), 3.36(\mathrm{dq}, J=13.9,7.0 \mathrm{~Hz}, 1 \mathrm{H}), 1.28(\mathrm{~d}, J=6.9 \mathrm{~Hz}$, 3H). ${ }^{13} \mathbf{C}\left\{{ }^{1} \mathbf{H}\right\}$ NMR (126 MHz, CDCl 3$): \delta 177.7(\mathrm{C}=\mathrm{O}), 160.3(\mathrm{C}), 146.8(\mathrm{C}), 144.2(\mathrm{C}), 130.6$ (C), $129.2(\mathrm{C}), 127.6(2 \mathrm{CH}), 127.0(\mathrm{CH}), 122.0(\mathrm{CH}), 114.4(2 \mathrm{CH}), 110.4(\mathrm{CH}), 83.0(\mathrm{C}), 61.7$ $\left(\mathrm{CH}_{2}\right), 55.5\left(\mathrm{CH}_{3}\right), 15.5\left(\mathrm{CH}_{3}\right)$. FT-IR (neat, $\left.\mathbf{c m}^{-1}\right)$ : 3231, 2929, 1733, 1622, 1606, 1525, 1510, 1338, 1253, 1177. HRMS (ESI $\left.{ }^{+}\right): m / z$ calcd. for $\mathrm{C}_{17} \mathrm{H}_{16} \mathrm{~N}_{2} \mathrm{O}_{5} \mathrm{Na}[\mathrm{M}+\mathrm{Na}]^{+}$351.0952, found 351.0950 . 
<smiles>CCOC1(C)C(=O)N(C)c2ccccc21</smiles>

General procedure A was followed with 5a $(64.5 \mathrm{mg}, 0.40 \mathrm{mmol})$ and ethanol $(2.5 \mathrm{~mL})$. Purification was performed with flash column chromatography over silica gel $\left(20 \mathrm{~g} \mathrm{SiO}_{2}\right.$, heptane/EtOAc, $100 / 0$ to 50/50, gradient) to afford 6a as a light yellow oil $(50.7 \mathrm{mg}$, 0.247 mmol, 62\%). ${ }^{1} \mathbf{H}$ NMR (400 MHz, CDCl 3$): \delta 7.32(\mathrm{dd}, J=10.2,4.2 \mathrm{~Hz}, 2 \mathrm{H}), 7.10(\mathrm{t}, J$ $=7.6 \mathrm{~Hz}, 1 \mathrm{H}), 6.83(\mathrm{~d}, J=8.2 \mathrm{~Hz}, 1 \mathrm{H}), 3.20(\mathrm{~s}, 3 \mathrm{H}), 3.15(\mathrm{dd}, J=14.7,7.6 \mathrm{~Hz}, 1 \mathrm{H}), 3.03(\mathrm{dd}$, $J=14.7,7.6 \mathrm{~Hz}, 1 \mathrm{H}), 1.54(\mathrm{~s}, 3 \mathrm{H}), 1.12(\mathrm{t}, J=7.0 \mathrm{~Hz}, 3 \mathrm{H}) .{ }^{13} \mathbf{C}\left\{{ }^{1} \mathbf{H}\right\} \mathbf{N M R}(\mathbf{1 0 0} \mathbf{M H z}, \mathbf{C D C l})$ : $\delta 177.0(\mathrm{C}=\mathrm{O}), 143.3(\mathrm{C}), 129.6(\mathrm{CH}), 129.5(\mathrm{C}), 123.7(\mathrm{CH}), 123.2(\mathrm{CH}), 108.4(\mathrm{CH}), 79.1$ (C), $61.0\left(\mathrm{CH}_{2}\right), 26.2\left(\mathrm{CH}_{3}\right), 24.3\left(\mathrm{CH}_{3}\right), 15.5\left(\mathrm{CH}_{3}\right)$. HRMS (ESI $\left.{ }^{+}\right): \mathrm{m} / z$ calcd. for $\mathrm{C}_{12} \mathrm{H}_{15} \mathrm{NO}_{2} \mathrm{Na}[\mathrm{M}+\mathrm{Na}]^{+} 228.0995$, found 228.0993. Spectral and physical data are in accordance with literature. ${ }^{17}$

\section{3-Ethoxy-3-methyl-1-phenylindolin-2-one $6 \mathrm{~b}$}<smiles>CCOC1(C)C(=O)N(c2ccccc2)c2ccccc21</smiles>

6b

General procedure A was followed with $\mathbf{5 b}(89.3 \mathrm{mg}, 0.40 \mathrm{mmol})$ and ethanol $(2.5 \mathrm{~mL})$. Purification was performed with flash column chromatography over silica gel $\left(20 \mathrm{~g} \mathrm{SiO}_{2}\right.$, heptane/EtOAc, 100/0 to 50/50, gradient) to afford $\mathbf{6 b}$ as a white solid (79.6 mg, $0.298 \mathrm{mmol}$, 74\%). ${ }^{1} \mathbf{H}$ NMR (500 MHz, CDCl $): \delta 7.52(\mathrm{dd}, J=10.6,5.1 \mathrm{~Hz}, 2 \mathrm{H}), 7.42(\mathrm{dd}, J=10.7,4.6$ $\mathrm{Hz}, 4 \mathrm{H}), 7.28-7.24(\mathrm{~m}, 1 \mathrm{H}), 7.15(\mathrm{td}, J=7.5,0.7 \mathrm{~Hz}, 1 \mathrm{H}), 6.84(\mathrm{~d}, J=7.9 \mathrm{~Hz}, 1 \mathrm{H}), 3.35$ (dq, $J$ $=13.9,7.0 \mathrm{~Hz}, 1 \mathrm{H}), 3.21(\mathrm{dq}, J=14.1,7.0 \mathrm{~Hz}, 1 \mathrm{H}), 1.68(\mathrm{~s}, 3 \mathrm{H}), 1.19(\mathrm{t}, J=7.0 \mathrm{~Hz}, 3 \mathrm{H})$.

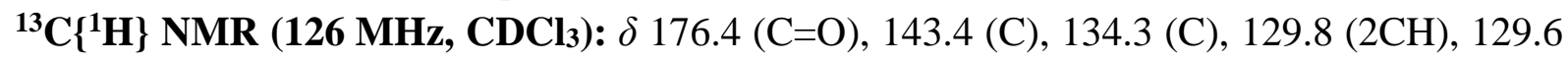
$(\mathrm{CH}), 129.4(\mathrm{C}), 128.3(\mathrm{CH}), 126.6(2 \mathrm{CH}), 124.1(\mathrm{CH}), 123.7(\mathrm{CH}), 109.8(\mathrm{CH}), 79.2(\mathrm{C}), 61.2$ $\left(\mathrm{CH}_{2}\right), 24.6\left(\mathrm{CH}_{3}\right), 15.6\left(\mathrm{CH}_{3}\right)$. FT-IR (neat, $\left.\mathbf{~ c m}^{-1}\right)$ : 3055, 2976, 2923, 2877, 1720, 1607, 1595, 1496, 1455, 1370, 1202, 1182. HRMS (ESI $\left.{ }^{+}\right): m / z$ calcd. for $\mathrm{C}_{17} \mathrm{H}_{17} \mathrm{NO}_{2} \mathrm{Na}[\mathrm{M}+\mathrm{Na}]^{+}$ 290.1152 , found 290.1151 . 


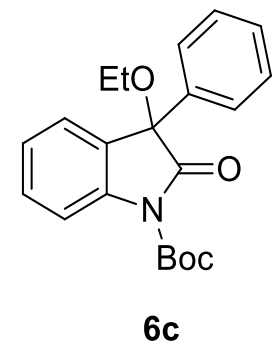

General procedure A was followed with $\mathbf{5 c}(124 \mathrm{mg}, 0.40 \mathrm{mmol})$ and ethanol $(2.5 \mathrm{~mL})$. Purification was performed with flash column chromatography over silica gel $\left(20 \mathrm{~g} \mathrm{SiO}_{2}\right.$, heptane/EtOAc, 100/0 to 50/50, gradient) to afford $\mathbf{6 c}$ as a white solid (55.6 mg, $0.157 \mathrm{mmol}$, 40\%). ${ }^{1} \mathbf{H}$ NMR (700 MHz, CDCl $): \delta 7.98(\mathrm{~d}, J=8.2 \mathrm{~Hz}, 1 \mathrm{H}), 7.44(\mathrm{td}, J=7.5,1.5 \mathrm{~Hz}, 1 \mathrm{H})$, $7.36(\mathrm{dd}, J=8.0,1.5 \mathrm{~Hz}, 2 \mathrm{H}), 7.33-7.28(\mathrm{~m}, 4 \mathrm{H}), 7.25(\mathrm{td}, J=7.5,0.6 \mathrm{~Hz}, 1 \mathrm{H}), 3.44(\mathrm{dq}, J=$ 14.0, 7.0 Hz, 1H), $3.30(\mathrm{dq}, J=14.0,7.0 \mathrm{~Hz}, 1 \mathrm{H}), 1.62(\mathrm{~s}, 9 \mathrm{H}), 1.25(\mathrm{t}, J=7.0 \mathrm{~Hz}, 3 \mathrm{H}) .{ }^{13} \mathbf{C}\left\{{ }^{1} \mathbf{H}\right\}$ NMR (176 MHz, CDCl3): $\delta 173.8(\mathrm{C}=\mathrm{O}), 149.3(\mathrm{C}=\mathrm{O}), 140.8(\mathrm{C}), 138.9(\mathrm{C}), 130.4(\mathrm{CH})$, $128.7(\mathrm{CH}), 128.6(2 \mathrm{CH}), 127.6(\mathrm{C}), 126.7(2 \mathrm{CH}), 125.7(\mathrm{CH}), 125.2(\mathrm{CH}), 115.5(\mathrm{CH}), 84.8$ (C), $83.4(\mathrm{C}), 61.4\left(\mathrm{CH}_{2}\right), 28.2\left(3 \mathrm{CH}_{3}\right), 15.5\left(\mathrm{CH}_{3}\right)$. FT-IR (neat, cm$\left.)^{-1}\right): 2923,2878,2853$, 1770, 1726, 1464, 1339, 1285, 1246, 1160. HRMS (ESI $\left.{ }^{+}\right): m / z$ calcd. for $\mathrm{C}_{21} \mathrm{H}_{23} \mathrm{NO}_{4} \mathrm{Na}$ $[\mathrm{M}+\mathrm{Na}]^{+} 376.1520$, found 376.1520 .

\section{3-Methoxy-3-(4-methoxyphenyl)indolin-2-one 7a}<smiles>COc1ccc(C2(OC)C(=O)Nc3ccccc32)cc1</smiles>

General procedure A was followed with $\mathbf{1 m}(95.7 \mathrm{mg}, 0.40 \mathrm{mmol})$ and methanol $(2.5 \mathrm{~mL})$. Purification was performed with flash column chromatography over silica gel $\left(20 \mathrm{~g} \mathrm{SiO}_{2}\right.$, heptane/EtOAc, 100/0 to 50/50, gradient) to afford 7a as a light yellow solid $(68.1 \mathrm{mg}$, 0.253 mmol, 63\%). ${ }^{1} \mathbf{H}$ NMR (400 MHz, CDCl $)$ : $\delta 9.29$ (brs, 1H), 7.36-7.28 (m, 3H), 7.24 (d, $J=7.3 \mathrm{~Hz}, 1 \mathrm{H}), 7.11(\mathrm{dd}, J=7.8,7.3 \mathrm{~Hz}, 1 \mathrm{H}), 6.95(\mathrm{~d}, J=7.8 \mathrm{~Hz}, 1 \mathrm{H}), 6.85(\mathrm{~d}, J=8.9 \mathrm{~Hz}$, 2H), 3.77 (s, 3H), 3.27 (s, 3H). ${ }^{13} \mathbf{C}\left\{{ }^{1} \mathbf{H}\right\}$ NMR (100 MHz, CDCl3): $\delta 178.5(\mathrm{C}=\mathrm{O}), 159.8(\mathrm{C})$, $141.9(\mathrm{C}), 130.6(\mathrm{C}), 130.2(\mathrm{CH}), 128.4(\mathrm{C}), 127.8(2 \mathrm{CH}), 126.1(\mathrm{CH}), 123.3(\mathrm{CH}), 114.0$ $(2 \mathrm{CH}), 111.0(\mathrm{CH}), 84.2(\mathrm{C}), 55.4\left(\mathrm{CH}_{3}\right), 53.3\left(\mathrm{CH}_{3}\right)$. FT-IR (neat, $\left.\mathbf{c m}^{-1}\right)$ : 3177, 2927, 2818 , 1720, 1690, 1618, 1605, 1508, 1471, 1255, 1219. HRMS (ESI $\left.{ }^{+}\right): m / z$ calcd. For $\mathrm{C}_{16} \mathrm{H}_{15} \mathrm{NO}_{3} \mathrm{Na}$ $[\mathrm{M}+\mathrm{Na}]^{+}$292.0945, found 292.0935. 


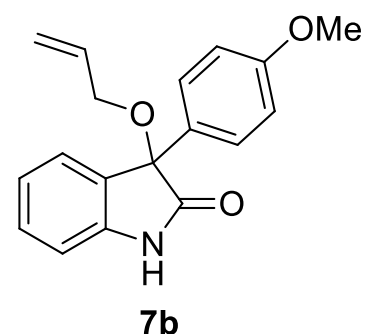

General procedure A was followed with $1 \mathrm{~m}(95.7 \mathrm{mg}, 0.40 \mathrm{mmol})$ and allyl alcohol $(2.5 \mathrm{~mL})$. Purification was performed with flash column chromatography over silica gel $\left(20 \mathrm{~g} \mathrm{SiO}_{2}\right.$, heptane/EtOAc, 100/0 to 50/50, gradient) to afford $\mathbf{7 b}$ as a light yellow solid (40.0 mg, 0.135 mmol, 34\%). ${ }^{\mathbf{1}} \mathbf{H}$ NMR (400 MHz, CDCl $): \delta 8.60$ (brs, $\left.1 \mathrm{H}\right), 7.35$ (d, $\left.J=8.9 \mathrm{~Hz}, 2 \mathrm{H}\right)$, 7.34.-7.29 (m, 1H), $7.27(\mathrm{~d}, J=6.4 \mathrm{~Hz}, 1 \mathrm{H}), 7.11(\mathrm{dd}, J=7.8,7.3 \mathrm{~Hz}, 1 \mathrm{H}), 6.94(\mathrm{~d}, J=7.8 \mathrm{~Hz}$, $1 \mathrm{H}), 6.85(\mathrm{~d}, J=8.9 \mathrm{~Hz}, 2 \mathrm{H}), 6.00-5.89(\mathrm{~m}, 1 \mathrm{H}), 5.32-5.25(\mathrm{~m}, 1 \mathrm{H}), 5.16-5.12(\mathrm{~m}, 1 \mathrm{H}), 3.97$ $(\mathrm{dd}, J=11.8,5.5 \mathrm{~Hz}, 1 \mathrm{H}), 3.83(\mathrm{dd}, J=11.8,5.5 \mathrm{~Hz}, 1 \mathrm{H}), 3.78(\mathrm{~s}, 3 \mathrm{H}) .{ }^{13} \mathbf{C}\left\{{ }^{1} \mathbf{H}\right\} \mathbf{N M R}$ (100 MHz, CDCl3): $\delta 178.1(\mathrm{C}=\mathrm{O}), 159.9(\mathrm{C}), 141.6(\mathrm{C}), 134.3(\mathrm{CH}), 130.7(\mathrm{C}), 130.2(\mathrm{CH})$, $128.9(\mathrm{C}), 127.9(2 \mathrm{CH}), 126.2(\mathrm{CH}), 123.4(\mathrm{CH}), 117.2\left(\mathrm{CH}_{2}\right), 114.2(2 \mathrm{CH}), 110.8(\mathrm{CH}), 83.5$ (C), $66.8\left(\mathrm{CH}_{2}\right), 55.4\left(\mathrm{CH}_{3}\right)$. FT-IR (neat, $\left.\mathbf{c m}^{-1}\right): 3177,2916,1720,1688,1617,1605,1509$, 1470, 1254, 1215, 1171, 1109. HRMS (ESI $\left.{ }^{+}\right): m / z$ calcd. for $\mathrm{C}_{18} \mathrm{H}_{17} \mathrm{NO}_{3} \mathrm{Na}[\mathrm{M}+\mathrm{Na}]^{+}$ 318.1101 , found 318.1112 .

\section{3-(Benzyloxy)-3-(4-methoxyphenyl)indolin-2-one 7c}<smiles>COc1ccc(C2(OCc3ccccc3)C(=O)Nc3ccccc32)cc1</smiles>

General procedure A was followed with $\mathbf{1 m}(95.7 \mathrm{mg}, 0.40 \mathrm{mmol})$ and benzyl alcohol $(2.5 \mathrm{~mL})$. Purification was performed with flash column chromatography over silica gel $\left(20 \mathrm{~g} \mathrm{SiO}_{2}\right.$, heptane/EtOAc, 100/0 to 50/50, gradient) to afford 7c as a light yellow solid $(90.1 \mathrm{mg}$, 0.261 mmol, 65\%). ${ }^{1} \mathbf{H}$ NMR (400 MHz, CDCl $): \delta 9.22$ (brs, 1H), 7.43-7.36 (m, 4H), 7.35$7.28(\mathrm{~m}, 4 \mathrm{H}), 7.27(\mathrm{~d}, J=7.5 \mathrm{~Hz}, 1 \mathrm{H}), 7.12(\mathrm{dd}, J=7.7,7.5 \mathrm{~Hz}, 1 \mathrm{H}), 6.98(\mathrm{~d}, J=7.7 \mathrm{~Hz}, 1 \mathrm{H})$, $6.86(\mathrm{~d}, J=8.8 \mathrm{~Hz}, 2 \mathrm{H}), 4.51(\mathrm{~d}, J=10.6 \mathrm{~Hz}, 1 \mathrm{H}), 4.33(\mathrm{~d}, J=10.6 \mathrm{~Hz}, 1 \mathrm{H}), 3.77(\mathrm{~s}, 3 \mathrm{H})$. ${ }^{13} \mathbf{C}\left\{{ }^{1} \mathbf{H}\right\}$ NMR (100 MHz, CDCl3): $\delta 178.3(\mathrm{C}=\mathrm{O}), 159.9(\mathrm{C}), 141.8(\mathrm{C}), 137.9(\mathrm{C}), 130.8(\mathrm{C})$, $130.3(\mathrm{CH}), 128.8(\mathrm{C}), 128.4(2 \mathrm{CH}), 128.0(2 \mathrm{CH}), 127.9(2 \mathrm{CH}), 127.8(\mathrm{CH}), 126.1(\mathrm{CH}), 123.4$ $(\mathrm{CH}), 114.0(2 \mathrm{CH}), 111.0(\mathrm{CH}), 83.4(\mathrm{C}), 67.7\left(\mathrm{CH}_{2}\right), 55.4\left(\mathrm{CH}_{3}\right)$. FT-IR (neat, $\left.\mathbf{~ c m}^{-1}\right)$ : 3195 , 3087, 3060, 2928, 2836, 1709, 1615, 1605, 1508, 1469, 1244, 1212, 1170, 1106, 1087, 1026. HRMS (ESI $\left.{ }^{+}\right): m / z$ calcd. for $\mathrm{C}_{22} \mathrm{H}_{19} \mathrm{NO}_{3} \mathrm{Na}[\mathrm{M}+\mathrm{Na}]^{+} 368.1258$, found 368.1265. 


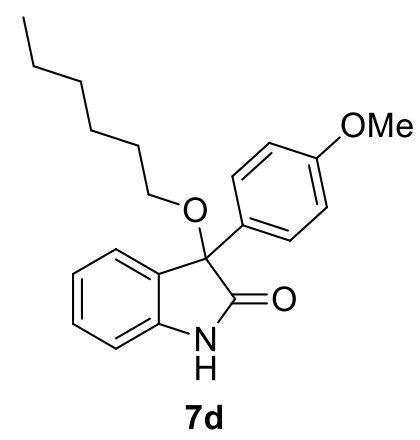

General procedure A was followed with $1 \mathrm{~m}(95.7 \mathrm{mg}, 0.40 \mathrm{mmol})$ and 1-hexanol $(1.0 \mathrm{~mL}$, 20.0 equiv.) in $\mathrm{MeCN}$ ( $5.0 \mathrm{~mL}$ ). Purification was performed with flash column chromatography over silica gel (20 $\mathrm{g} \mathrm{SiO}_{2}$, heptane/EtOAc, 100/0 to 50/50, gradient) to afford 7d as a light brown solid (71.0 mg, 0.209 mmol, 52\%). ${ }^{1} \mathbf{H}$ NMR (600 MHz, CDCl 3 ): $\delta 8.74$ (brs, 1H), 7.33 $(\mathrm{d}, J=8.9 \mathrm{~Hz}, 2 \mathrm{H}), 7.30(\mathrm{td}, J=7.7,1.2 \mathrm{~Hz}, 1 \mathrm{H}), 7.23(\mathrm{~d}, J=7.2 \mathrm{~Hz}, 1 \mathrm{H}), 7.10(\mathrm{td}, J=7.5$, $0.8 \mathrm{~Hz}, 1 \mathrm{H}), 6.94(\mathrm{~d}, J=7.8 \mathrm{~Hz}, 1 \mathrm{H}), 6.84(\mathrm{~d}, J=8.9 \mathrm{~Hz}, 4 \mathrm{H}), 3.77(\mathrm{~s}, 3 \mathrm{H}), 3.21(\mathrm{dt}, J=8.2$, $6.8 \mathrm{~Hz}, 2 \mathrm{H}), 3.21(\mathrm{dt}, J=8.2,6.8 \mathrm{~Hz}, 1 \mathrm{H}), 1.62(\mathrm{dt}, J=13.7,6.8 \mathrm{~Hz}, 2 \mathrm{H}), 1.39-1.33(\mathrm{~m}, 2 \mathrm{H})$, 1.30-1.22 (m, 4H), $0.86(\mathrm{t}, J=7.1 \mathrm{~Hz}, 3 \mathrm{H}) .{ }^{\mathbf{1 3}} \mathbf{C}\left\{{ }^{\mathbf{1}} \mathbf{H}\right\}$ NMR (150 MHz, CDCl $\left.\mathbf{3}\right): \delta 178.3(\mathrm{C}=\mathrm{O})$, $159.8(\mathrm{C}), 141.6(\mathrm{C}), 131.2(\mathrm{C}), 130.0(\mathrm{CH}), 129.3(\mathrm{C}), 127.8(2 \mathrm{CH}), 126.1(\mathrm{CH}), 123.3(\mathrm{CH})$, $113.9(2 \mathrm{CH}), 110.7(\mathrm{CH}), 83.5(\mathrm{C}), 66.6\left(\mathrm{CH}_{2}\right), 55.4\left(\mathrm{CH}_{3}\right), 31.7\left(\mathrm{CH}_{2}\right), 30.0\left(\mathrm{CH}_{2}\right), 25.9\left(\mathrm{CH}_{2}\right)$, $22.7\left(\mathrm{CH}_{2}\right), 14.2\left(\mathrm{CH}_{3}\right)$. FT-IR (neat, $\left.\mathbf{~ c m}^{-1}\right): 3171,2930,2867,1721,1689,1619,1606,1509$, 1467, 1217. HRMS (ESI $\left.{ }^{+}\right): \mathrm{m} / z$ calcd. for $\mathrm{C}_{21} \mathrm{H}_{25} \mathrm{NO}_{3} \mathrm{Na}[\mathrm{M}+\mathrm{Na}]^{+} 362.1727$, found 362.1727 .

\section{3-(But-2-yn-1-yloxy)-3-(4-methoxyphenyl)indolin-2-one 7e}

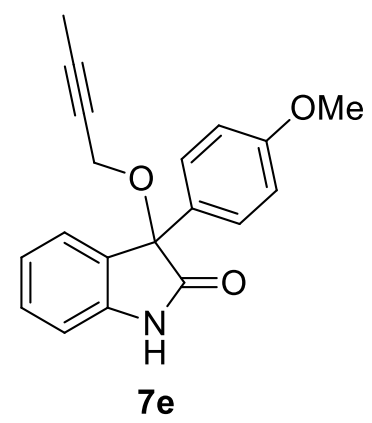

General procedure A was followed with $\mathbf{1 m}(95.7 \mathrm{mg}, 0.40 \mathrm{mmol})$ and 2-butyn-1-ol (0.60 mL, 20.0 equiv.) in $\mathrm{MeCN}$ (5.0 mL). Purification was performed with flash column chromatography over silica gel (20 $\mathrm{g} \mathrm{SiO}_{2}$, heptane/EtOAc, 100/0 to 50/50, gradient) to afford 7e as a light yellow solid (65.0 mg, $0.211 \mathrm{mmol}, 53 \%) .{ }^{1} \mathbf{H}$ NMR (700 MHz, CDCl 3 ): $\delta 9.08$ (brs, 1H), 7.34 $(\mathrm{d}, J=8.9 \mathrm{~Hz}, 2 \mathrm{H}), 7.31(\mathrm{td}, J=7.7,1.1 \mathrm{~Hz}, 1 \mathrm{H}), 7.28(\mathrm{~d}, J=7.3 \mathrm{~Hz}, 1 \mathrm{H}), 7.09(\mathrm{dd}, J=11.0$, $4.0 \mathrm{~Hz}, 1 \mathrm{H}), 6.96(\mathrm{~d}, J=7.7 \mathrm{~Hz}, 1 \mathrm{H}), 6.83(\mathrm{~d}, J=8.9 \mathrm{~Hz}, 2 \mathrm{H}), 4.10(\mathrm{dq}, J=14.2,2.3 \mathrm{~Hz}, 1 \mathrm{H})$, $4.01(\mathrm{dq}, J=14.2,2.3 \mathrm{~Hz}, 1 \mathrm{H}), 3.76$ (s, 3H), 1.76 (s, 3H). ${ }^{\mathbf{1 3}} \mathbf{C}\left\{{ }^{1} \mathbf{H}\right\}$ NMR (176 MHz, CDCl $)$ : $\delta 177.9(\mathrm{C}=\mathrm{O}), 159.9(\mathrm{C}), 141.7(\mathrm{C}), 130.4(\mathrm{CH}), 130.3(\mathrm{C}), 128.1(\mathrm{C}), 128.0(2 \mathrm{CH}), 126.4$ $(\mathrm{CH}), 123.3(\mathrm{CH}), 114.0(2 \mathrm{CH}), 110.9(\mathrm{CH}), 83.4(\mathrm{C}), 83.2(\mathrm{C}), 74.9(\mathrm{C}), 55.4\left(\mathrm{CH}_{3}\right), 54.6$ $\left(\mathrm{CH}_{2}\right), 3.8\left(\mathrm{CH}_{3}\right)$. FT-IR (neat, $\left.\mathbf{c m}^{-1}\right)$ : 3208, 2961, 2921, 1720, 1681, 1619, 1508, 1466, 1215. HRMS (ESI ${ }^{+}$): $m / z$ calcd. for $\mathrm{C}_{19} \mathrm{H}_{17} \mathrm{NO}_{3} \mathrm{Na}[\mathrm{M}+\mathrm{Na}]^{+} 330.1101$, found 330.1103 . 


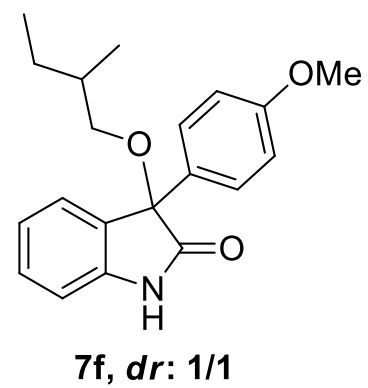

General procedure A was followed with $1 \mathbf{m}(95.7 \mathrm{mg}, 0.40 \mathrm{mmol})$ and DL-2-methyl-1-butanol (2.5 mL). Purification was performed with flash column chromatography over silica gel (20 g $\mathrm{SiO}_{2}$, heptane/EtOAc, 100/0 to 50/50, gradient) to afford $7 \mathbf{f}$ as a light yellow solid (87.0 mg, $0.267 \mathrm{mmol}, 67 \%, d r: 1 / 1) .{ }^{1} \mathbf{H}$ NMR (400 $\mathbf{~ M H z}, \mathbf{C D C l}_{3}$, mixture of diastereoisomers): $\delta 9.00$ (brs, 1H), 7.34 (d, $J=8.7 \mathrm{~Hz}, 2 \mathrm{H}), 7.30$ (t, $J=7.8 \mathrm{~Hz}, 1 \mathrm{H}), 7.21$ (d, $J=7.3 \mathrm{~Hz}, 1 \mathrm{H}), 7.09$ (dd, $J=7.8,7.3 \mathrm{~Hz}, 1 \mathrm{H}), 6.94(\mathrm{~d}, J=7.8 \mathrm{~Hz}, 1 \mathrm{H}), 6.84(\mathrm{~d}, J=8.9 \mathrm{~Hz}, 2 \mathrm{H}), 3.77$ (s, 3H), 3.25 (ddd, $J=21.0,8.0,6.0 \mathrm{~Hz}, 1 \mathrm{H}), 3.02(\mathrm{ddd}, J=21.0,8.0,6.5 \mathrm{~Hz}, 1 \mathrm{H}), 1.75-1.66(\mathrm{~m}, 1 \mathrm{H}), 1.56-1.43$ (m, 1H), 1.21-1.09 (m, 1H), $0.92(\mathrm{dd}, 6.7,2.8 \mathrm{~Hz}, 3 \mathrm{H}), 0.88-0.82(\mathrm{~m}, 3 \mathrm{H}) .{ }^{13} \mathbf{C}\left\{{ }^{1} \mathbf{H}\right\}$ NMR (100 MHz, $\mathbf{C D C l}_{3}$, mixture of diastereoisomers): $\delta 178.6(\mathrm{C}=\mathrm{O}), 159.7(\mathrm{C}), 141.7(\mathrm{C}), 131.4$ (C), $130.0(\mathrm{CH}), 129.3(\mathrm{C}), 129.3(2 \mathrm{CH}), 127.7(\mathrm{CH}), 123.3(\mathrm{CH}), 113.9(2 \mathrm{CH}), 110.7(\mathrm{CH})$, $83.4(\mathrm{C}), 70.3\left(\mathrm{CH}_{2}, \mathrm{~d} 1\right), 70.2\left(\mathrm{CH}_{2}, \mathrm{~d} 2\right), 55.4\left(\mathrm{CH}_{3}\right), 35.4(\mathrm{CH}, \mathrm{d} 1), 35.3(\mathrm{CH}, \mathrm{d} 2), 26.2\left(\mathrm{CH}_{2}\right.$, d1), $26.2\left(\mathrm{CH}_{2}, \mathrm{~d} 2\right), 16.8\left(\mathrm{CH}_{3}, \mathrm{~d} 1\right), 16.6\left(\mathrm{CH}_{3}, \mathrm{~d} 2\right), 11.4\left(\mathrm{CH}_{3}, \mathrm{~d} 1\right), 11.3\left(\mathrm{CH}_{3}, \mathrm{~d} 2\right)$. FT-IR (neat, $\mathbf{~ c m}^{-1}$ ): 3208, 2960, 2908, 2873, 1722, 1687, 1618, 1607, 1508, 1467, 1254, 1215, 1171. HRMS (ESI ${ }^{+}$): $m / z$ calcd. for $\mathrm{C}_{20} \mathrm{H}_{23} \mathrm{NO}_{3} \mathrm{Na}[\mathrm{M}+\mathrm{Na}]^{+} 348.1571$, found 348.1580.

\section{3-(4-Methoxyphenyl)-3-(2-((trimethylsilyl)oxy)ethoxy)indolin-2-one 7g}

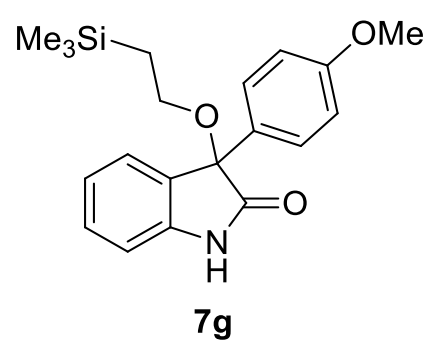

General procedure A was followed with $\mathbf{1 m}(95.7 \mathrm{mg}, 0.40 \mathrm{mmol})$ and 2-(trimethylsilyl)ethanol (1.19 mL, 20.0 equiv.) in $\mathrm{MeCN}(5.0 \mathrm{~mL})$. Purification was performed with flash column chromatography over silica gel (20 g SiO 2 , heptane/EtOAc, 100/0 to 50/50, gradient) to afford $\mathbf{7 g}$ as a light yellow solid (115.0 mg, $0.323 \mathrm{mmol}, 81 \%)$. ${ }^{\mathbf{1}} \mathbf{H}$ NMR (400 $\left.\mathbf{M H z}, \mathbf{C D C l}_{3}\right): \delta 8.38$ (brs, $1 \mathrm{H}), 7.35-7.28(\mathrm{~m}, 3 \mathrm{H}), 7.24(\mathrm{~d}, J=7.2 \mathrm{~Hz}, 1 \mathrm{H}), 7.12$ (dd, $J=7.8,7.2 \mathrm{~Hz}, 1 \mathrm{H}), 6.93$ (d, $J=7.8 \mathrm{~Hz}, 1 \mathrm{H}), 6.84(\mathrm{~d}, J=8.9 \mathrm{~Hz}, 2 \mathrm{H}), 3.77(\mathrm{~s}, 3 \mathrm{H}), 3.51(\mathrm{td}, J=8.8,6.4 \mathrm{~Hz}, 1 \mathrm{H}), 3.34(\mathrm{td}$, $J=8.8,6.9 \mathrm{~Hz}, 1 \mathrm{H}), 1.04-0.93(\mathrm{~m}, 2 \mathrm{H}),-0.03(\mathrm{~s}, 9 \mathrm{H}) .{ }^{13} \mathbf{C}\left\{{ }^{1} \mathbf{H}\right\}$ NMR (100 MHz, CDCl 3$): \delta$ $178.2(\mathrm{C}=\mathrm{O}), 159.8(\mathrm{C}), 141.5(\mathrm{C}), 131.2(\mathrm{C}), 130.0(\mathrm{CH}), 129.3(\mathrm{C}), 127.8(2 \mathrm{CH}), 126.1(\mathrm{CH})$, $123.3(\mathrm{CH}), 113.9(2 \mathrm{CH}), 110.6(\mathrm{CH}), 83.5(\mathrm{C}), 62.9\left(\mathrm{CH}_{2}\right), 55.4\left(\mathrm{CH}_{3}\right), 18.7\left(\mathrm{CH}_{2}\right),-1.1$ $\left(3 \mathrm{CH}_{3}\right)$. FT-IR (neat, $\left.\mathbf{c m}^{-1}\right)$ : 3084, 2955, 2894, 1714, 1614, 1604, 1508, 1469, 1275, 1252 , 1174. HRMS (ESI $\left.{ }^{+}\right): \mathrm{m} / z$ calcd. for $\mathrm{C}_{20} \mathrm{H}_{25} \mathrm{NO}_{3} \mathrm{SiNa}[\mathrm{M}+\mathrm{Na}]^{+} 378.1496$, found 378.1508. 


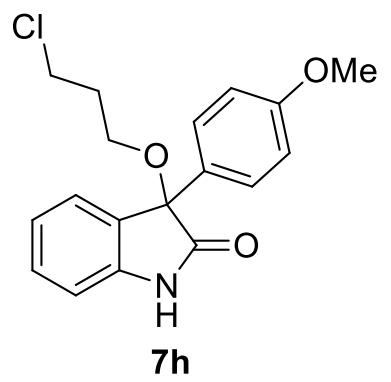

General procedure A was followed with $\mathbf{1 m}(95.7 \mathrm{mg}, 0.40 \mathrm{mmol})$ and 3-chloro-1-propanol (2.5 mL). Purification was performed with flash column chromatography over silica gel (20 g $\mathrm{SiO}_{2}$, heptane/EtOAc, $100 / 0$ to $50 / 50$, gradient) to afford $\mathbf{7 h}$ as a light yellow solid $(78.1 \mathrm{mg}$,

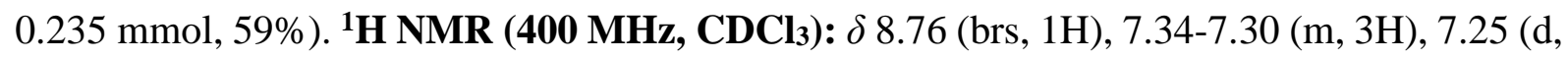
$J=7.7 \mathrm{~Hz}, 1 \mathrm{H}), 7.11(\mathrm{dd}, J=7.8,7.7 \mathrm{~Hz}, 1 \mathrm{H}), 6.95(\mathrm{~d}, J=7.8 \mathrm{~Hz}, 1 \mathrm{H}), 6.85(\mathrm{~d}, J=8.9 \mathrm{~Hz}$, 2H), $3.77(\mathrm{~s}, 3 \mathrm{H}), 3.71(\mathrm{t}, J=6.5 \mathrm{~Hz}, 2 \mathrm{H}), 3.54-3.49(\mathrm{~m}, 1 \mathrm{H}), 3.44-3.38(\mathrm{~m}, 1 \mathrm{H}), 2.14-2.02(\mathrm{~m}$, 2H). ${ }^{13} \mathbf{C}\left\{{ }^{1} \mathbf{H}\right\}$ NMR (100 MHz, CDCl 3$): \delta 178.3(\mathrm{C}=\mathrm{O}), 159.9(\mathrm{C}), 141.6(\mathrm{C}), 130.7(\mathrm{C}), 130.2$ $(\mathrm{CH}), 128.7(\mathrm{C}), 127.8(2 \mathrm{CH}), 126.1(\mathrm{CH}), 123.4(\mathrm{CH}), 114.0(2 \mathrm{CH}), 110.9(\mathrm{CH}), 83.6(\mathrm{C})$, $62.0\left(\mathrm{CH}_{2}\right), 55.4\left(\mathrm{CH}_{3}\right), 41.9\left(\mathrm{CH}_{2}\right), 33.0\left(\mathrm{CH}_{2}\right)$. FT-IR (neat, cm-1): 3171, 2957, 1727, 1684, $1617,1508,1468,1250,1214,1170$. $\mathbf{H R M S}\left(\mathbf{E S I}^{+}\right): \mathrm{m} / z$ calcd. for $\mathrm{C}_{18} \mathrm{H}_{18} \mathrm{NClO}_{3} \mathrm{Na}[\mathrm{M}+\mathrm{Na}]^{+}$ 354.0867 , found 354.0867 .

3-(4-Methoxyphenyl)-3-phenethoxyindolin-2-one 7i<smiles>COc1ccc(C2(OCCc3ccccc3)C(=O)Nc3ccccc32)cc1</smiles>

General procedure A was followed with $\mathbf{1 m}(95.7 \mathrm{mg}, 0.40 \mathrm{mmol})$ and 2-phenylethyl alcohol $(0.50 \mathrm{~mL}, 10.0$ equiv.) in $\mathrm{MeCN}(5.0 \mathrm{~mL})$. Purification was performed with flash column chromatography over silica gel (20 $\mathrm{g} \mathrm{SiO}_{2}$, heptane/EtOAc, 100/0 to 50/50, gradient) to afford 7i as a brown solid (51.0 mg, $0.142 \mathrm{mmol}, 36 \%)$. ${ }^{\mathbf{1}} \mathbf{H}$ NMR (500 MHz, CDCl $): \delta 8.69$ (brs, 1H), 7.32-7.26 (m, 3H), 7.24 (d, $J=7.5 \mathrm{~Hz}, 2 \mathrm{H}), 7.21-7.15(\mathrm{~m}, 3 \mathrm{H}), 7.05(\mathrm{qd}, J=7.4,0.9 \mathrm{~Hz}$, 2H), $6.91(\mathrm{~d}, J=7.8 \mathrm{~Hz}, 1 \mathrm{H}), 6.83(\mathrm{~d}, J=8.9 \mathrm{~Hz}, 2 \mathrm{H}), 3.77(\mathrm{~s}, 3 \mathrm{H}), 3.66(\mathrm{td}, J=7.9,6.2 \mathrm{~Hz}$, 1H), $3.43(\mathrm{dd}, J=15.8,7.5 \mathrm{~Hz}, 1 \mathrm{H}), 3.00-2.91(\mathrm{~m}, 2 \mathrm{H}) .{ }^{13} \mathbf{C}\left\{{ }^{1} \mathbf{H}\right\}$ NMR (126 MHz, CDCl $\left.\mathbf{3}\right): \delta$ $178.1(\mathrm{C}=\mathrm{O}), 159.8(\mathrm{C}), 141.5(\mathrm{C}), 138.7(\mathrm{C}), 130.9(\mathrm{C}), 130.1(\mathrm{CH}), 129.2(2 \mathrm{CH}), 129.0(\mathrm{C})$, $128.4(2 \mathrm{CH}), 127.8(2 \mathrm{CH}), 126.3(\mathrm{CH}), 126.1(\mathrm{CH}), 123.4(\mathrm{CH}), 113.9(2 \mathrm{CH}), 110.6(\mathrm{CH})$, $83.6(\mathrm{C}), 66.5\left(\mathrm{CH}_{2}\right), 55.4\left(\mathrm{CH}_{3}\right), 36.6\left(\mathrm{CH}_{2}\right)$. FT-IR (neat, $\left.\mathbf{c m}^{-1}\right): 3291,2928,1724,1687$, 1687, 1619, 1506, 1452. HRMS (ESI $\left.{ }^{+}\right): m / z$ calcd. for $\mathrm{C}_{23} \mathrm{H}_{21} \mathrm{NO}_{3} \mathrm{Na}[\mathrm{M}+\mathrm{Na}]^{+} 383.1414$, found 383.1408 . 


\section{Procedures and Characterization data of 3,3-substituted oxindoles 8 and 9 (Scheme 3)}

\section{3-(4-Methoxyphenyl)-3-(2-oxo-2-phenylethyl)indolin-2-one 8}<smiles>COc1ccc(C2(CC(=O)c3ccccc3)C(=O)Nc3ccccc32)cc1</smiles>

8

With no precautions to exclude air or moisture, the ElectraSyn vial $(10 \mathrm{~mL})$ was charged with $1 \mathrm{~m}$ (95.7 mg, $0.40 \mathrm{mmol}$ ), Et 4 NOTs (121 mg, $0.40 \mathrm{mmol}, 1.0$ equiv.), 1-phenyl-1trimethylsilyloxyethylene ( $820 \mu \mathrm{L}, 10.00$ equiv.) and $\mathrm{MeCN}(5.0 \mathrm{~mL})$. The ElectraSyn vial cap equipped with anode (graphite) and cathode (graphite) were inserted into the mixture. The reaction mixture was electrolyzed at a constant current of $10 \mathrm{~mA}$ for $3 \mathrm{~h}$. The ElectraSyn vial cap was removed, and electrodes were rinsed with DCM $(2.0 \mathrm{~mL})$, which was combined with the crude mixture. Then, the crude mixture was concentrated under reduced pressure and purified by FC over silica gel (heptane/ethyl acetate, 100/0 to 50/50, gradient) to furnish the desired product 8 as a light yellow solid $(88.2 \mathrm{mg}, 0.247 \mathrm{mmol}, 62 \%)$.

${ }^{1}$ H NMR (400 MHz, CDCl $): \delta 8.21$ (brs, 1H), 7.91-7.84 (m, 2H), $7.53(\mathrm{t}, J=7.4 \mathrm{~Hz}, 1 \mathrm{H})$, $7.43-7.33(\mathrm{~m}, 4 \mathrm{H}), 7.24(\mathrm{dd}, J=13.0,5.4 \mathrm{~Hz}, 2 \mathrm{H}), 7.01(\mathrm{t}, J=7.5 \mathrm{~Hz}, 1 \mathrm{H}), 6.96(\mathrm{~d}, J=7.6 \mathrm{~Hz}$, $1 \mathrm{H}), 6.85(\mathrm{~d}, J=8.9 \mathrm{~Hz}, 2 \mathrm{H}), 4.15(\mathrm{~d}, J=18.0 \mathrm{~Hz}, 1 \mathrm{H}), 4.08(\mathrm{~d}, J=18.0 \mathrm{~Hz}, 1 \mathrm{H}), 3.77(\mathrm{~s}, 3 \mathrm{H})$. ${ }^{13} \mathbf{C}\left\{{ }^{1} \mathbf{H}\right\}$ NMR (100 MHz, CDCl 3$): \delta 196.0(\mathrm{C}=\mathrm{O}), 180.9(\mathrm{C}=\mathrm{O}), 159.2(\mathrm{C}), 141.9(\mathrm{C}), 136.5$ $(\mathrm{C}), 133.5(\mathrm{CH}), 132.5(\mathrm{C}), 131.4(\mathrm{C}), 128.7(2 \mathrm{CH}), 128.4(\mathrm{CH}), 128.2(2 \mathrm{CH}), 128.0(2 \mathrm{CH})$, $124.5(\mathrm{CH}), 122.3(\mathrm{CH}), 114.2(2 \mathrm{CH}), 111.3(\mathrm{CH}), 55.4\left(\mathrm{CH}_{3}\right), 53.0(\mathrm{C}), 47.0\left(\mathrm{CH}_{2}\right)$. FT-IR (neat, $\mathbf{~ c m}^{-1}$ ): 3132, 3058, 3007, 2963, 2838, 1708, 1671, 1616, 1506, 1472, 1218, 1183. HRMS $\left(\mathbf{E S I}^{+}\right): \mathrm{m} / z$ calcd. for $\mathrm{C}_{23} \mathrm{H}_{19} \mathrm{NO}_{3} \mathrm{Na}[\mathrm{M}+\mathrm{Na}]^{+} 380.1258$, found 380.1253 .

\section{3-Azido-3-(4-methoxyphenyl)indolin-2-one 9}

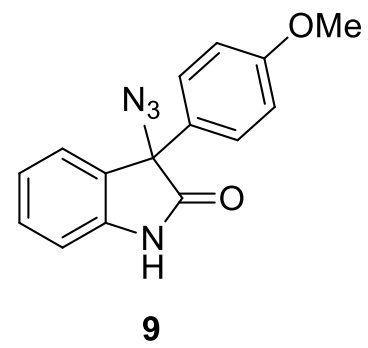

With no precautions to exclude air or moisture, the ElectraSyn vial (10 mL) was charged with $1 \mathrm{~m}(95.7 \mathrm{mg}, 0.40 \mathrm{mmol})$, Et 4 NOTs $(121 \mathrm{mg}, 0.40 \mathrm{mmol}, 1.0$ equiv.), azidotrimethylsilane ( $0.53 \mathrm{~mL}, 10.0$ equiv.) and $\mathrm{MeCN}(5.0 \mathrm{~mL})$. The ElectraSyn vial cap equipped with anode (graphite) and cathode (graphite) were inserted into the mixture. The reaction mixture was electrolyzed at a constant current of $10 \mathrm{~mA}$ for $3 \mathrm{~h}$. The ElectraSyn vial cap was removed, and 
electrodes were rinsed with DCM (2.0 mL), which was combined with the crude mixture. Then, the crude mixture was concentrated under reduced pressure and purified by FC over silica gel (heptane/ethyl acetate, 100/0 to 50/50, gradient) to furnish the desired product 9 as a light yellow oil (25.1 mg, $0.089 \mathrm{mmol}, 22 \%)$.

${ }^{1}$ H NMR (400 MHz, CDCl $): \delta 8.66$ (brs, $\left.1 \mathrm{H}\right), 7.37$ (d, $\left.J=8.8 \mathrm{~Hz}, 2 \mathrm{H}\right), 7.35-7.31$ (m, 1H), $7.29(\mathrm{~d}, J=7.4 \mathrm{~Hz}, 1 \mathrm{H}), 7.13(\mathrm{dd}, J=7.8,7.4 \mathrm{~Hz}, 1 \mathrm{H}), 6.98(\mathrm{~d}, J=7.8 \mathrm{~Hz}, 1 \mathrm{H}), 6.90(\mathrm{~d}, J=$ $8.8 \mathrm{~Hz}, 2 \mathrm{H}), 3.79$ (s, 3H). ${ }^{\mathbf{1 3}} \mathbf{C}\left\{{ }^{1} \mathbf{H}\right\}$ NMR (100 MHz, CDCl $\left.\mathbf{3}\right): \delta 176.1(\mathrm{C}=\mathrm{O}), 160.2(\mathrm{C}), 140.7$ (C), $130.5(\mathrm{CH}), 129.0(\mathrm{C}), 128.2(2 \mathrm{CH}), 128.1(\mathrm{C}), 125.6(\mathrm{CH}), 123.7(\mathrm{CH}), 114.5(2 \mathrm{CH})$, $111.0(\mathrm{CH}), 70.0(\mathrm{C}), 55.5\left(\mathrm{CH}_{3}\right)$. FT-IR (neat, $\left.\mathbf{c m}^{-1}\right)$ : 3234, 2956, 2932, 2837, 2096, 1716, 1508, 1470, 1249, 1177. HRMS (ESI $\left.{ }^{+}\right): m / z$ calcd. for $\mathrm{C}_{15} \mathrm{H}_{12} \mathrm{~N}_{4} \mathrm{O}_{2} \mathrm{Na}[\mathrm{M}+\mathrm{Na}]^{+} 303.0853$, found 303.0867 . 


\section{Additional Experiments with Different Type of Alkenes}

All these reactions were carried out on $0.40 \mathrm{mmol}$ scale following the general procedure $\mathrm{A}$ and are preliminary unoptimized results. The crude reaction mixture was analyzed by ${ }^{1} \mathrm{H} \mathrm{NMR}$ and purified by $\mathrm{FC}\left(10 \mathrm{~g} \mathrm{SiO}_{2}\right.$, heptane/ethyl acetate: $100 / 0$ to $70 / 30,35 \mathrm{CV}$ ).

Ketene Silyl Acetal<smiles>COc1ccc(C2C(=O)Nc3ccccc32)cc1</smiles>

$1 \mathrm{~m}$<smiles>C=C(OC)O[Ga]C(C)(C)C</smiles>

(10 eq)<smiles>COC(=O)CC1(c2ccc(OC)cc2)C(=O)Nc2ccccc21</smiles>

$17 \%$

Allylsilane<smiles>COc1ccc(C2C(=O)Nc3ccccc32)cc1</smiles>

$1 \mathrm{~m}$

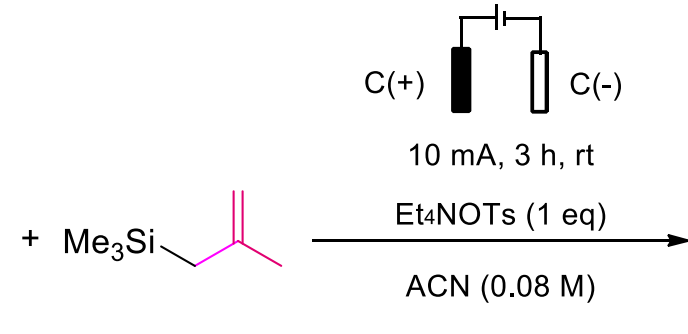

(10 eq)<smiles>C=C(C)C[C@]1(c2ccc(OC)cc2)C(=O)Nc2ccccc21</smiles>

$37 \%$ (52\% brsm)

Alkenyl Silane<smiles>COc1ccc(C2C(=O)Nc3ccccc32)cc1</smiles>

$1 \mathrm{~m}$<smiles>COC1=CCCC=C1</smiles>

(5 eq)<smiles>COc1ccc(C2(C3CCC=CC3=O)C(=O)Nc3ccccc32)cc1</smiles>

traces of product mainly SM recovered 


\section{BHT trapping experiment (Scheme 4)}

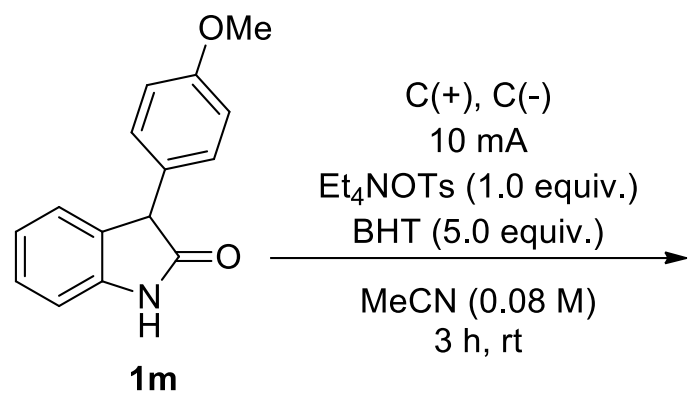<smiles>COc1ccc(C2(Cc3cc(CC(C)(C)C)c(O)c(CC(C)(C)C)c3)C(=O)Nc3ccccc32)cc1</smiles>

With no precautions to exclude air or moisture, the ElectraSyn vial $(10 \mathrm{~mL})$ was charged with 3-(4-methoxyphenyl)indolin-2-one $\mathbf{1 m}$ (95.7 mg, $0.40 \mathrm{mmol}, 1.0$ equiv.), Et ${ }_{4} \mathrm{NOTs}$ (121 mg, $0.40 \mathrm{mmol}, 1.0$ equiv.), 2,6-di-tert-butyl-4-methylphenol (441 mg, $2.0 \mathrm{mmol}, 5.0$ equiv.) and $\mathrm{MeCN}(5.0 \mathrm{~mL})$. The ElectraSyn vial cap equipped with anode (graphite) and cathode (graphite) were inserted into the mixture. The reaction mixture was electrolyzed at a constant current of $10 \mathrm{~mA}$ for $3 \mathrm{~h}$. The ElectraSyn vial cap was removed, and electrodes were rinsed with DCM (2.0 mL), which was combined with the crude mixture. Then, the crude mixture was concentrated under reduced pressure and purified by FC over silica gel (heptane/ethyl acetate, 100/0 to 50/50, gradient) to furnish the desired product $\mathbf{1 0}$ as a white solid (98.9 $\mathrm{mg}, 0.216$ mmol, 54\%).

${ }^{1}$ H NMR (400 MHz, CDCl $): \delta 8.54(\mathrm{~s}, 1 \mathrm{H}), 7.33(\mathrm{~d}, J=8.8 \mathrm{~Hz}, 2 \mathrm{H}), 7.07(\mathrm{td}, J=7.7,1.3 \mathrm{~Hz}$, $1 \mathrm{H}), 7.03-6.92(\mathrm{~m}, 2 \mathrm{H}), 6.80(\mathrm{~d}, J=8.8 \mathrm{~Hz}, 2 \mathrm{H}), 6.65(\mathrm{~d}, J=7.7 \mathrm{~Hz}, 1 \mathrm{H}), 6.56(\mathrm{~s}, 2 \mathrm{H}), 4.86(\mathrm{~s}$, $1 \mathrm{H}), 3.72(\mathrm{~s}, 3 \mathrm{H}), 3.45(\mathrm{~d}, J=12.9 \mathrm{~Hz}, 1 \mathrm{H}), 3.30(\mathrm{~d}, J=12.9 \mathrm{~Hz}, 1 \mathrm{H}), 1.13(\mathrm{~s}, 18 \mathrm{H}) .{ }^{13} \mathbf{C}\left\{{ }^{1} \mathbf{H}\right\}$ NMR (100 MHz, CDCl $)$ : $\delta 180.5$ (C=O), 159.0 (C), 152.5 (C), 141.1 (C), 134.9 (2C), 132.5 (C), $131.8(\mathrm{C}), 128.7(2 \mathrm{CH}), 128.0(\mathrm{CH}), 127.1(2 \mathrm{CH}), 126.2(\mathrm{CH}), 126.1(\mathrm{C}), 122.1(\mathrm{CH})$, $114.0(2 \mathrm{CH}), 109.8(\mathrm{CH}), 58.4(\mathrm{C}), 55.4\left(\mathrm{CH}_{3}\right), 44.2\left(\mathrm{CH}_{2}\right), 34.2(2 \mathrm{C}), 30.3\left(6 \mathrm{CH}_{3}\right) . \mathbf{H R M S}$ $\left(\mathbf{E S I}^{+}\right): \mathrm{m} / z$ calcd. for $\mathrm{C}_{30} \mathrm{H}_{35} \mathrm{NO}_{3} \mathrm{Na}[\mathrm{M}+\mathrm{Na}]^{+} 480.2510$, found 480.2513 . 


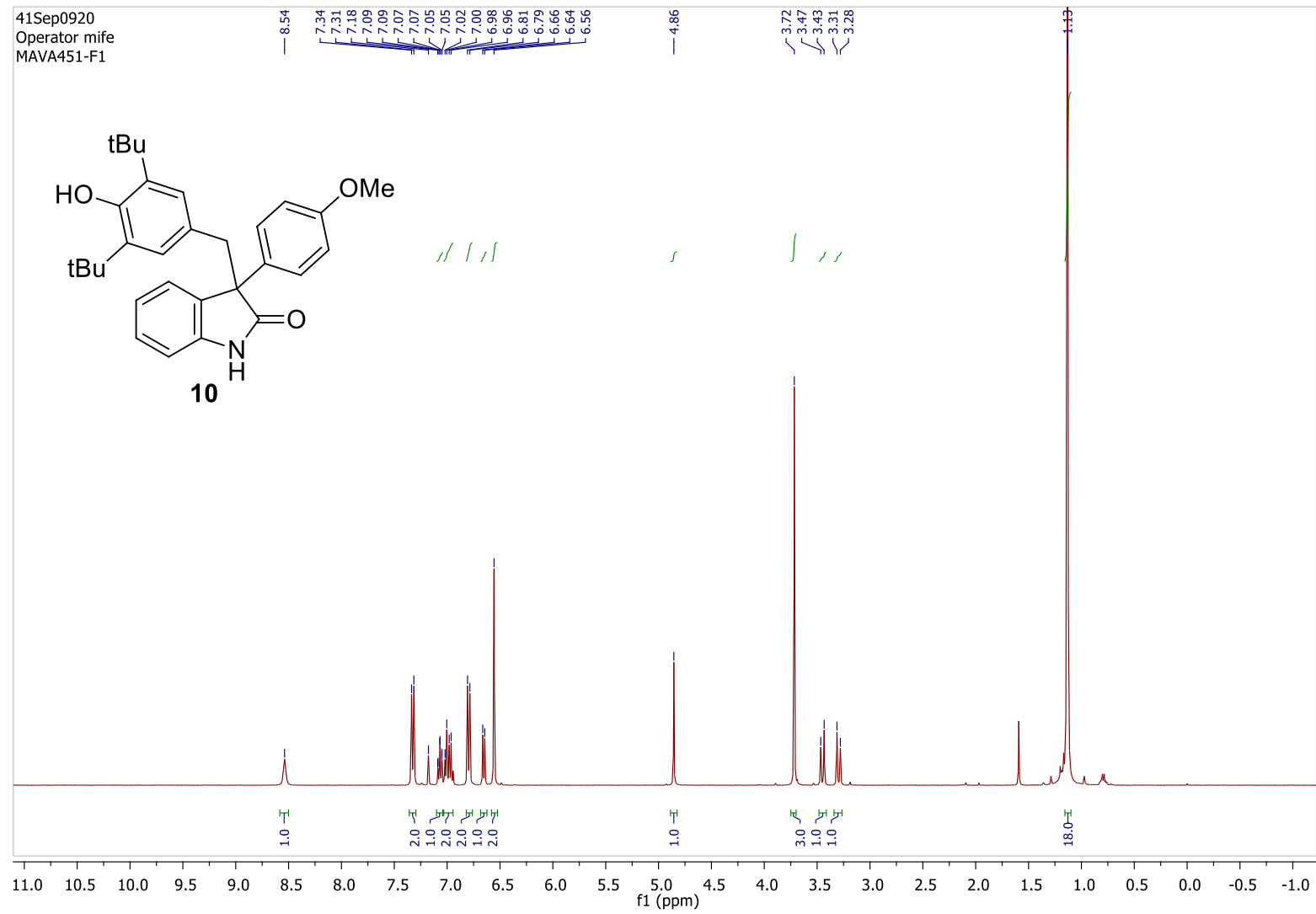

${ }^{1} \mathrm{H} \mathrm{NMR}$ in $\mathrm{CDCl}_{3}$ at $400 \mathrm{MHz}$

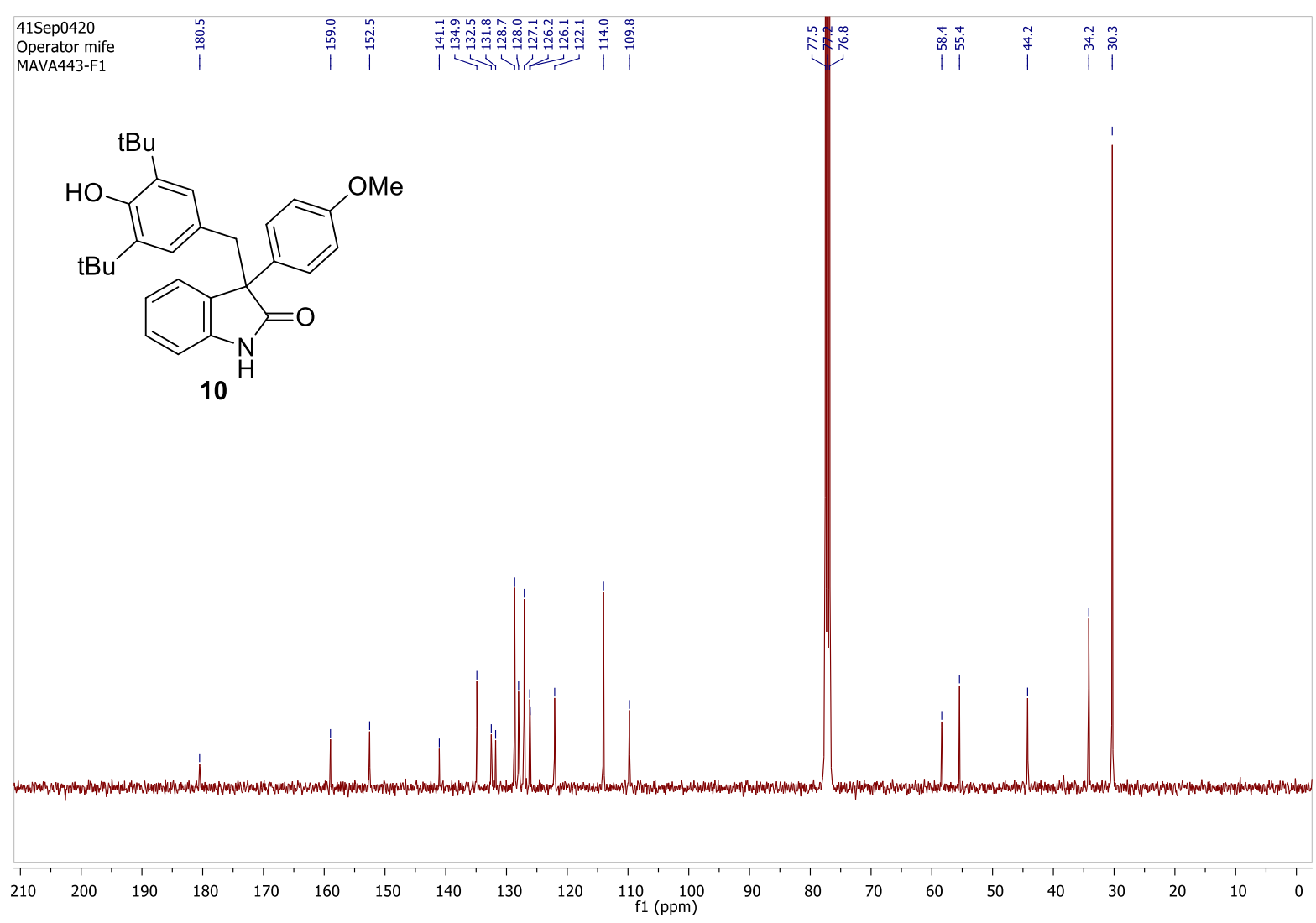

${ }^{13} \mathrm{C}\left\{{ }^{1} \mathrm{H}\right\} \mathrm{NMR}$ in $\mathrm{CDCl}_{3}$ at $100 \mathrm{MHz}$ 


\section{NMR Spectra}

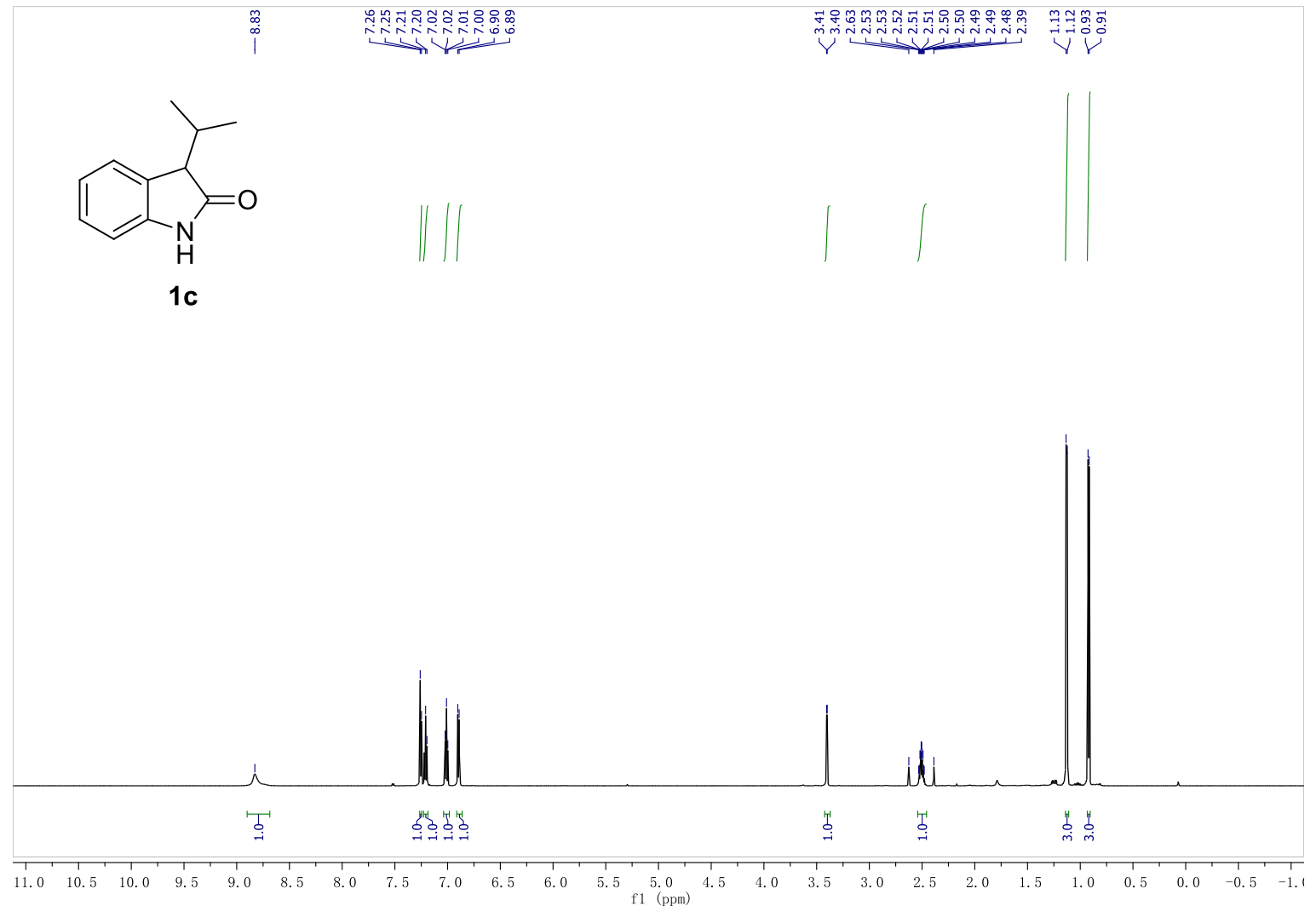

${ }^{1} \mathrm{H} \mathrm{NMR}$ in $\mathrm{CDCl}_{3}$ at $600 \mathrm{MHz}$

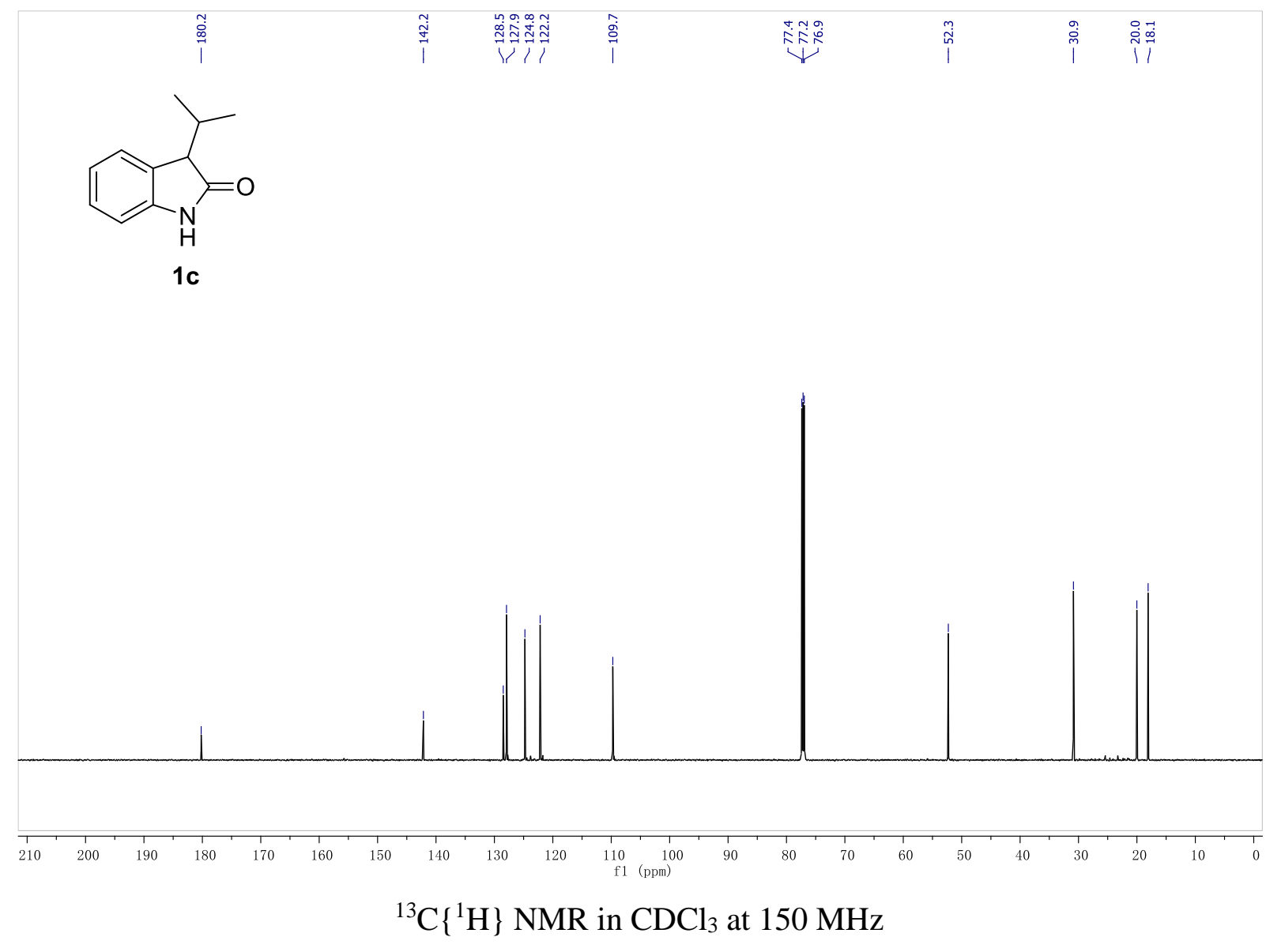



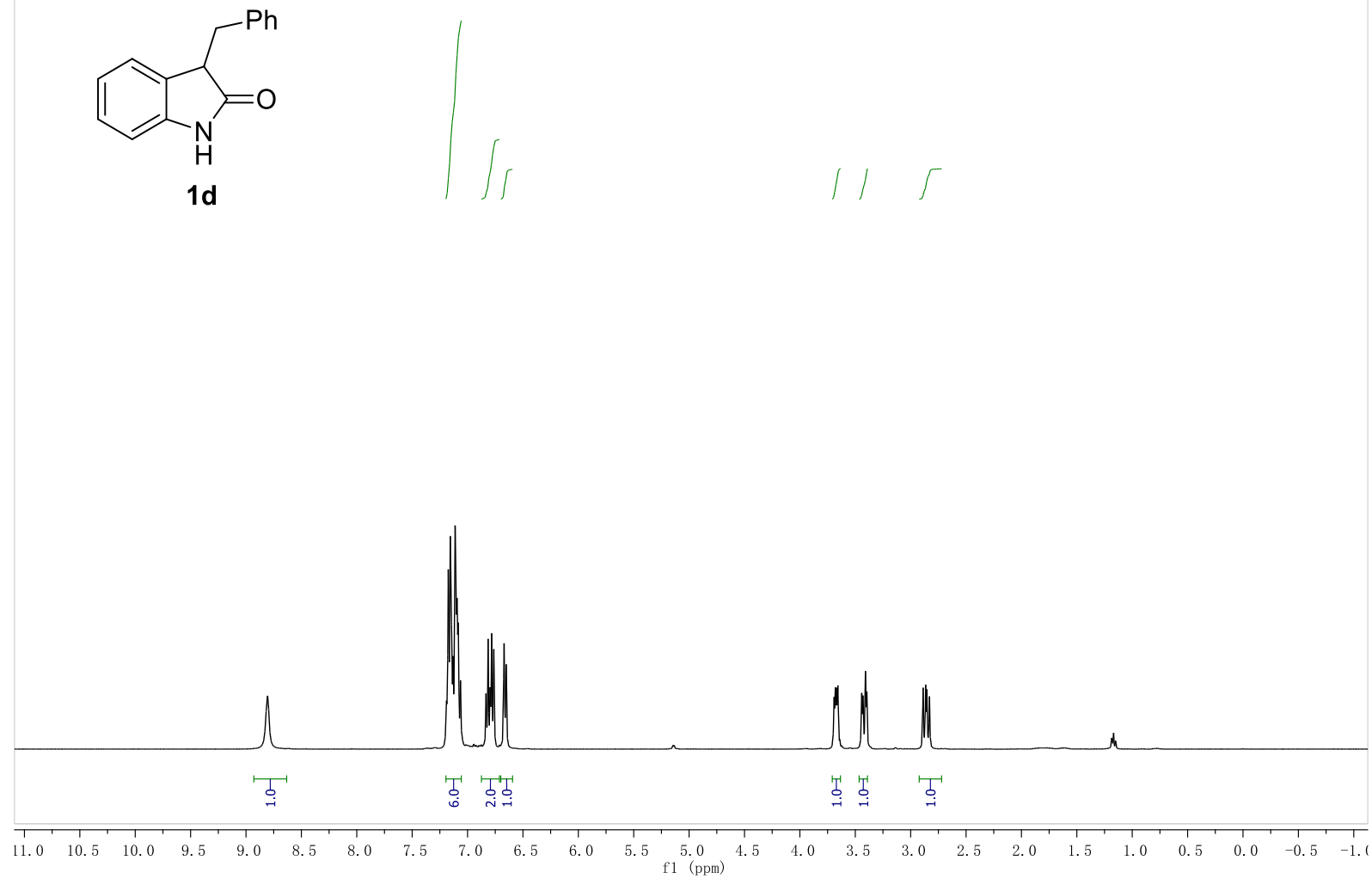

${ }^{1} \mathrm{H} \mathrm{NMR}$ in $\mathrm{CDCl}_{3}$ at $400 \mathrm{MHz}$

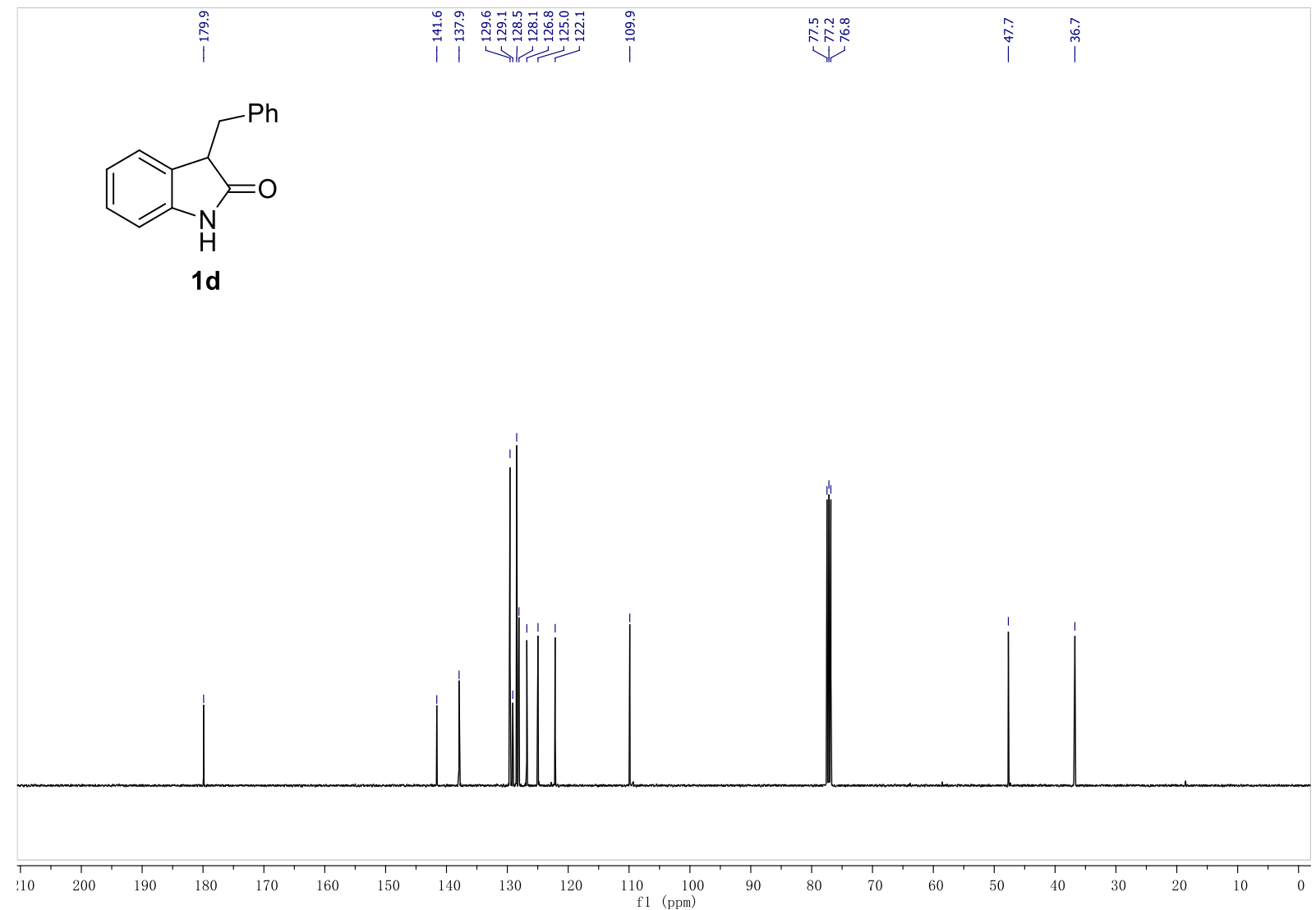

${ }^{13} \mathrm{C}\left\{{ }^{1} \mathrm{H}\right\} \mathrm{NMR}$ in $\mathrm{CDCl}_{3}$ at $100 \mathrm{MHz}$ 


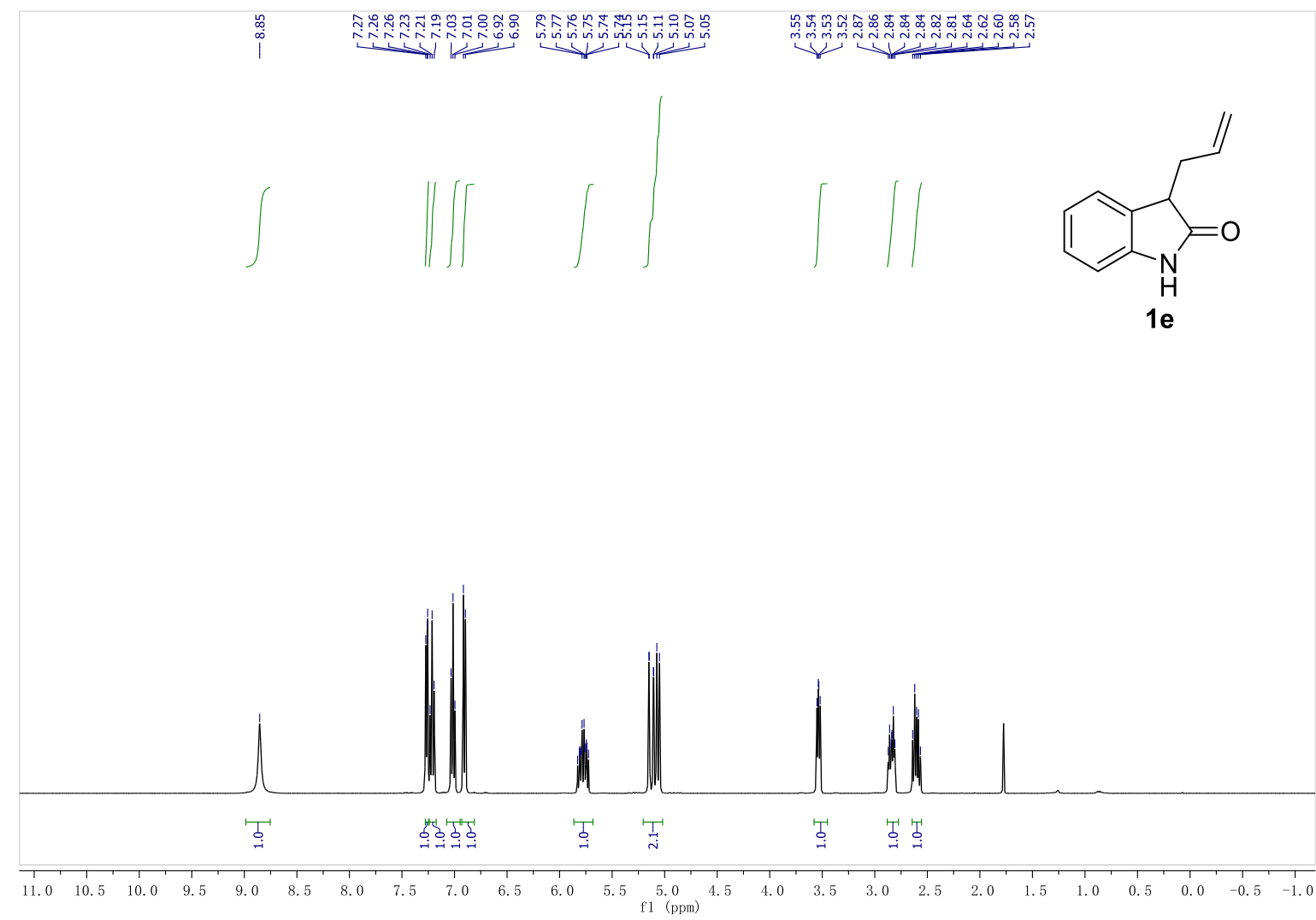

${ }^{1} \mathrm{H} \mathrm{NMR}$ in $\mathrm{CDCl}_{3}$ at $400 \mathrm{MHz}$

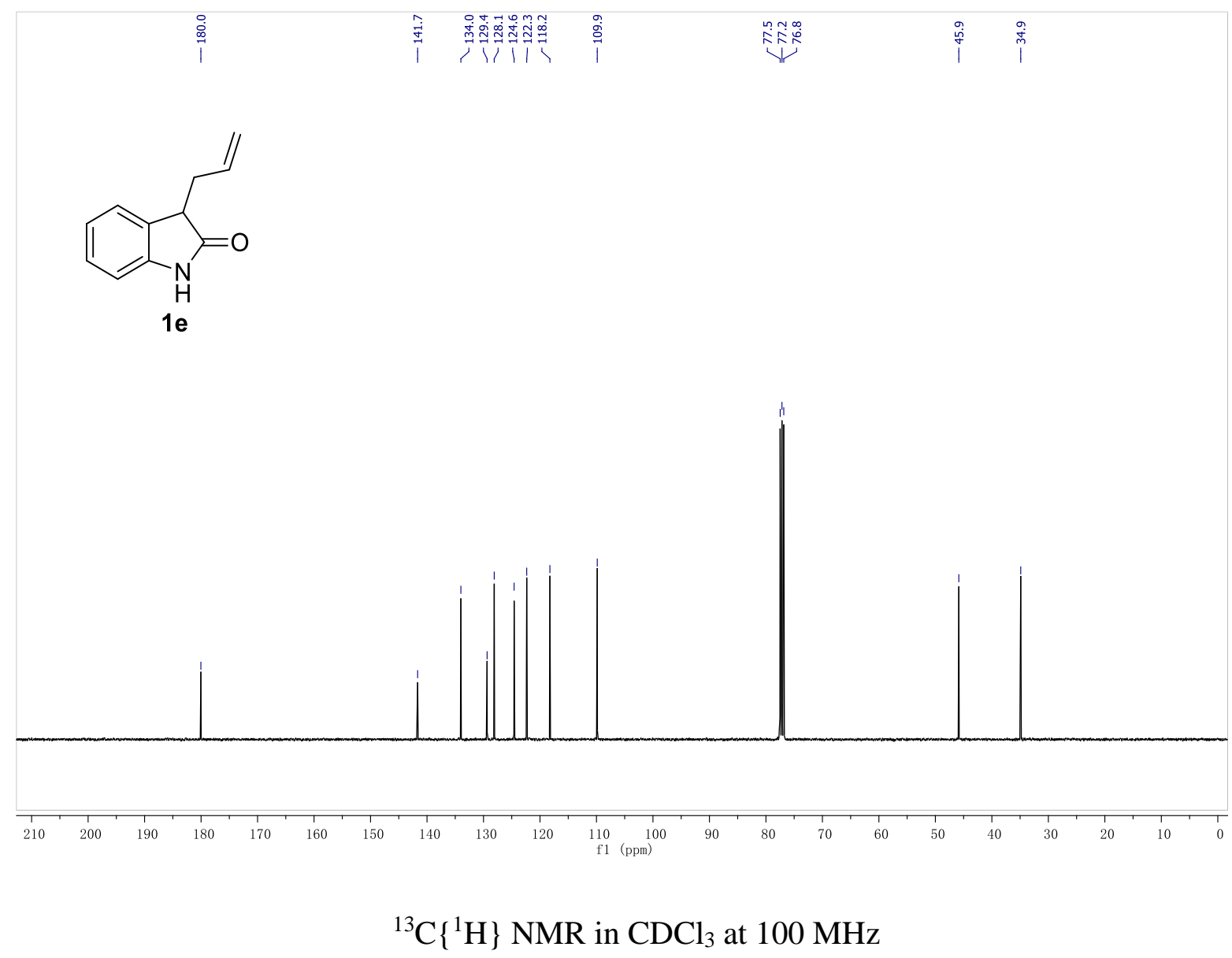




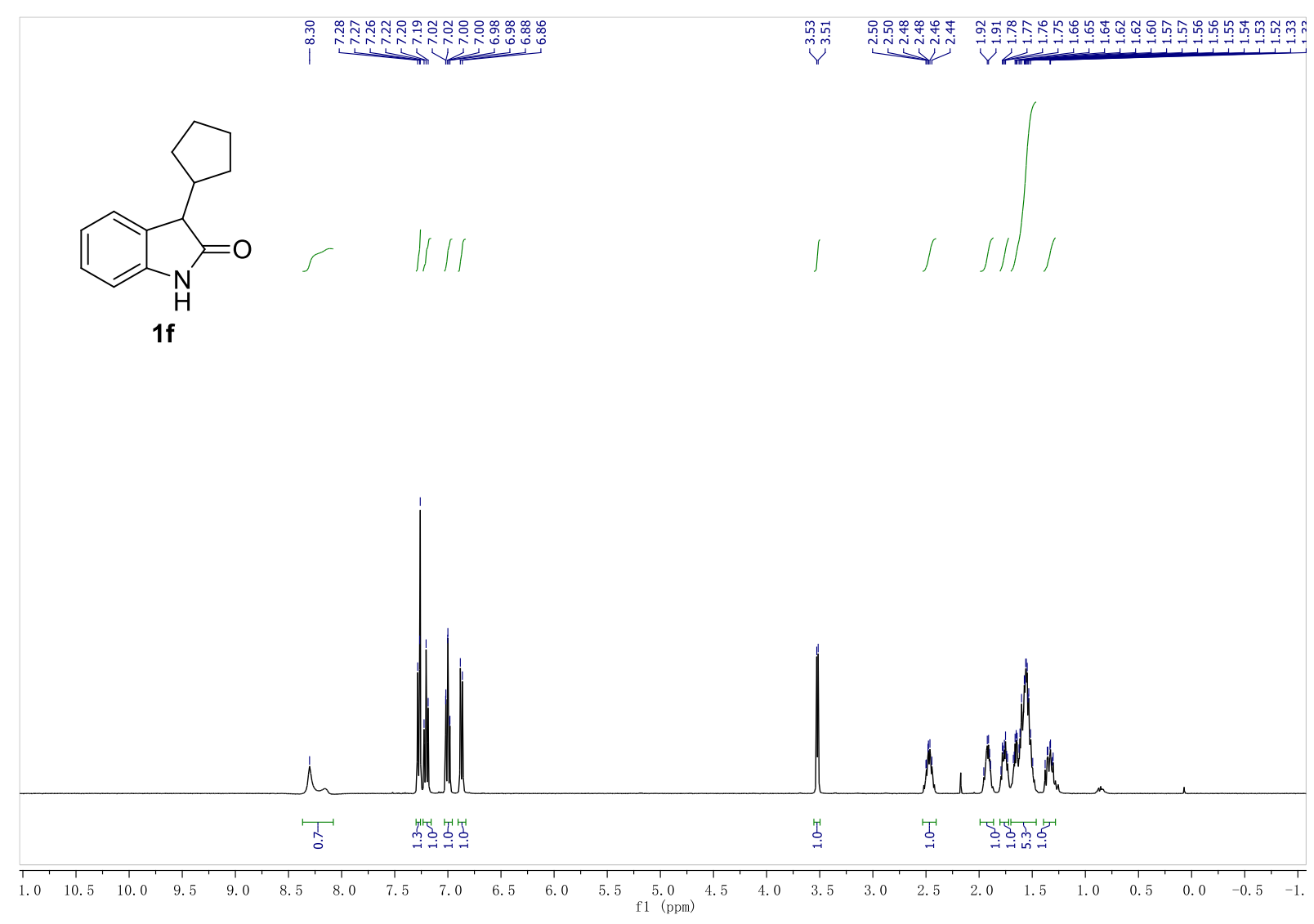

${ }^{1} \mathrm{H} \mathrm{NMR}$ in $\mathrm{CDCl}_{3}$ at $400 \mathrm{MHz}$

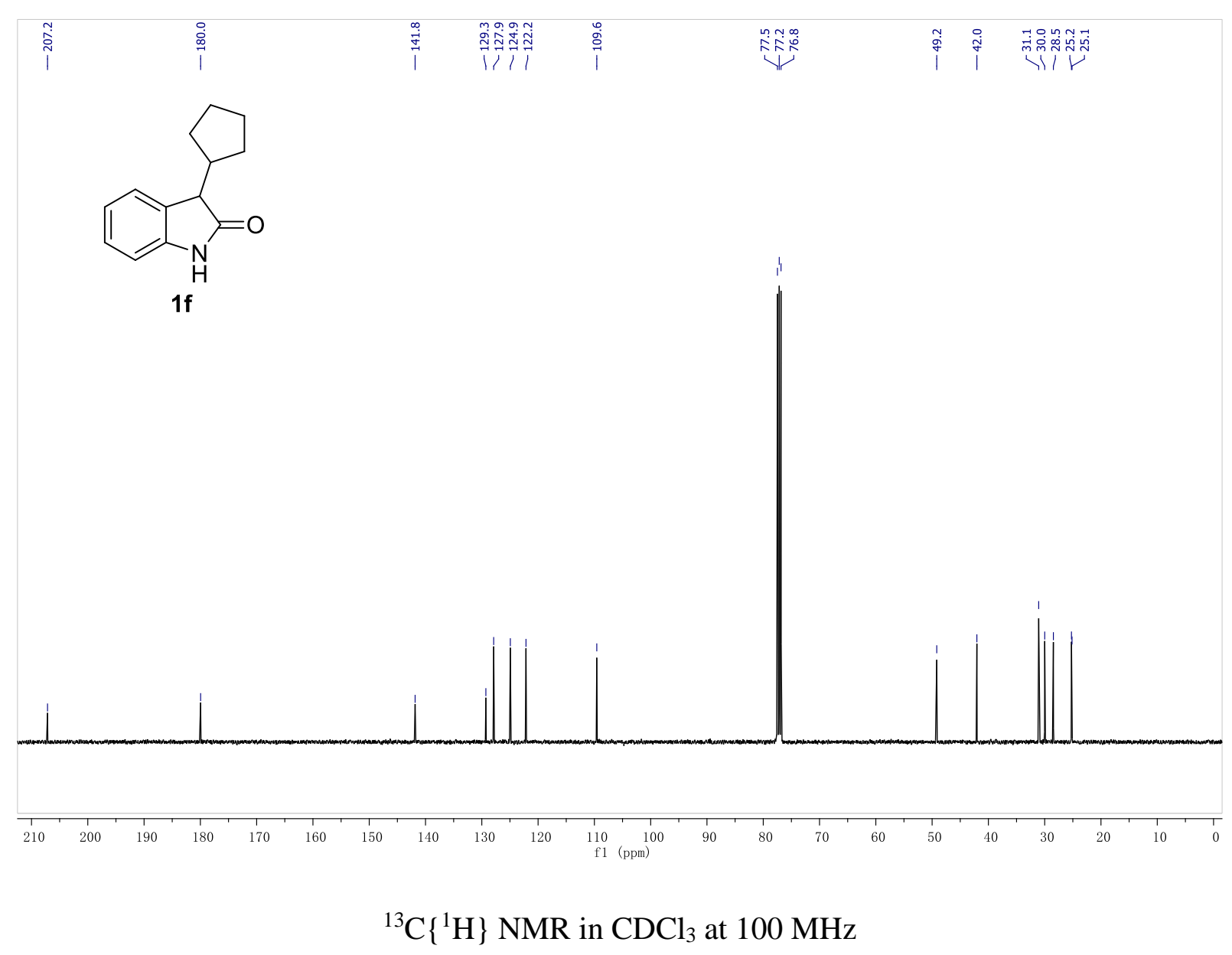




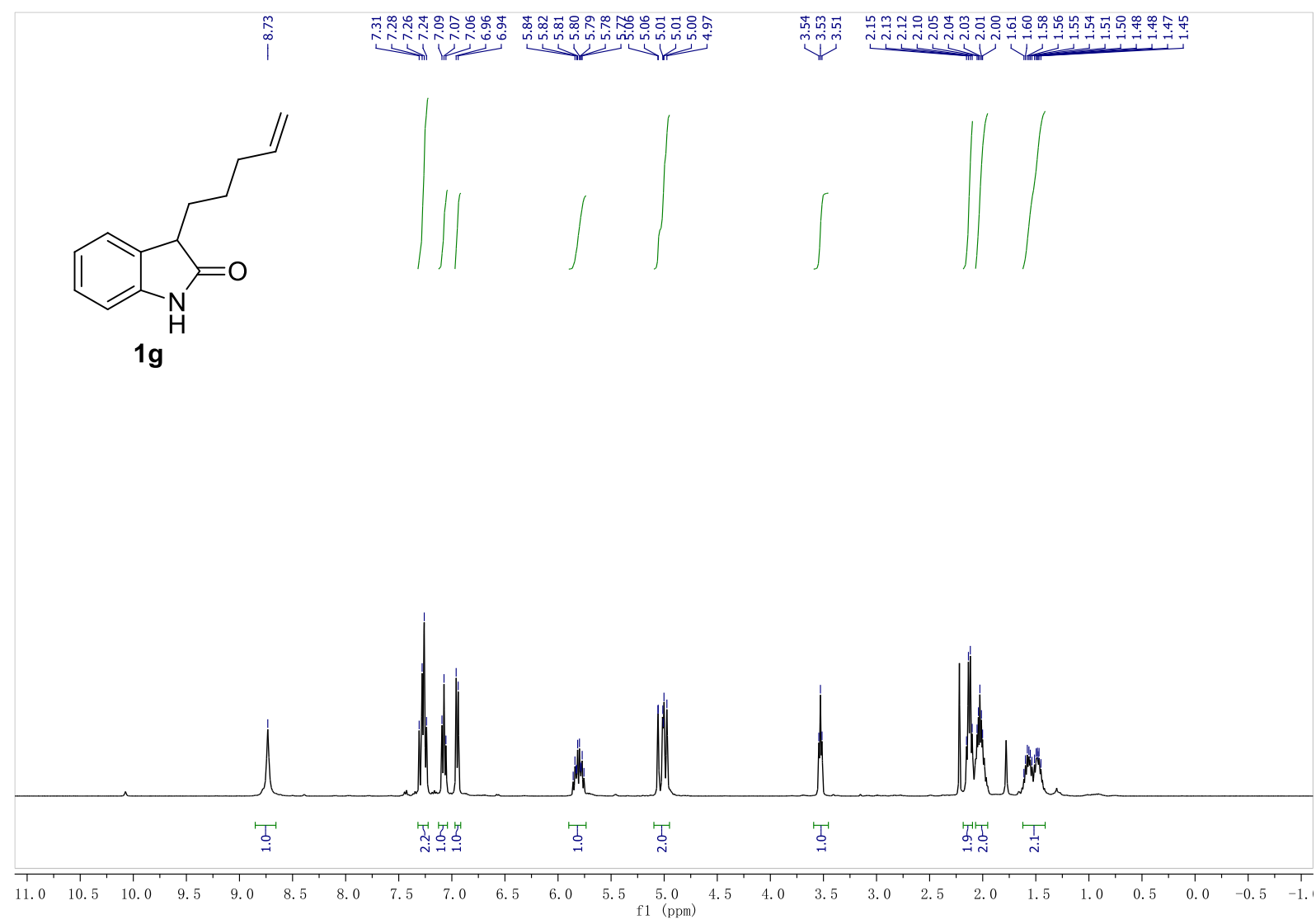

${ }^{1} \mathrm{H} \mathrm{NMR}$ in $\mathrm{CDCl}_{3}$ at $400 \mathrm{MHz}$

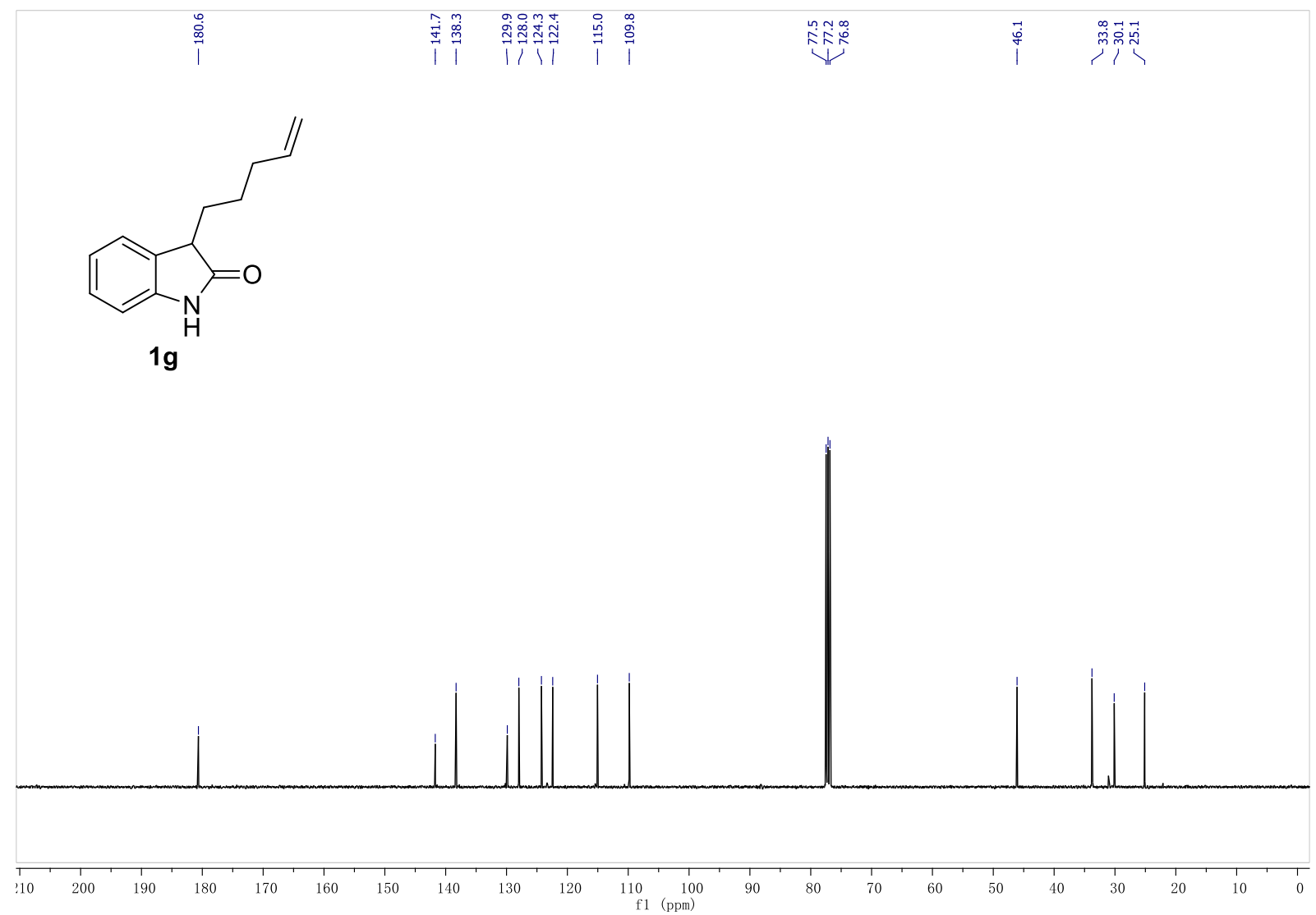

${ }^{13} \mathrm{C}\left\{{ }^{1} \mathrm{H}\right\} \mathrm{NMR}$ in $\mathrm{CDCl}_{3}$ at $100 \mathrm{MHz}$ 


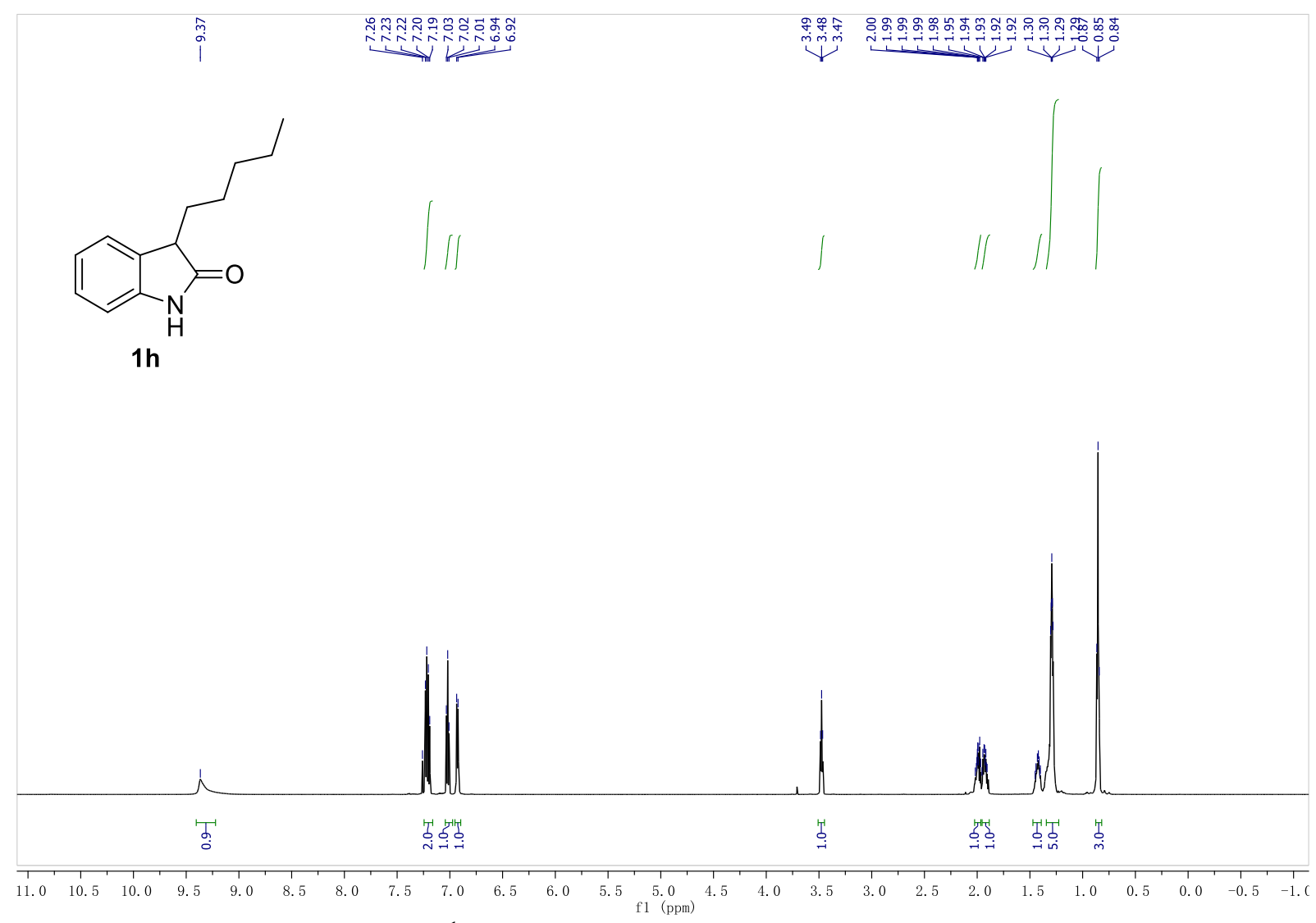

${ }^{1} \mathrm{H} \mathrm{NMR} \mathrm{in} \mathrm{CDCl}_{3}$ at $600 \mathrm{MHz}$

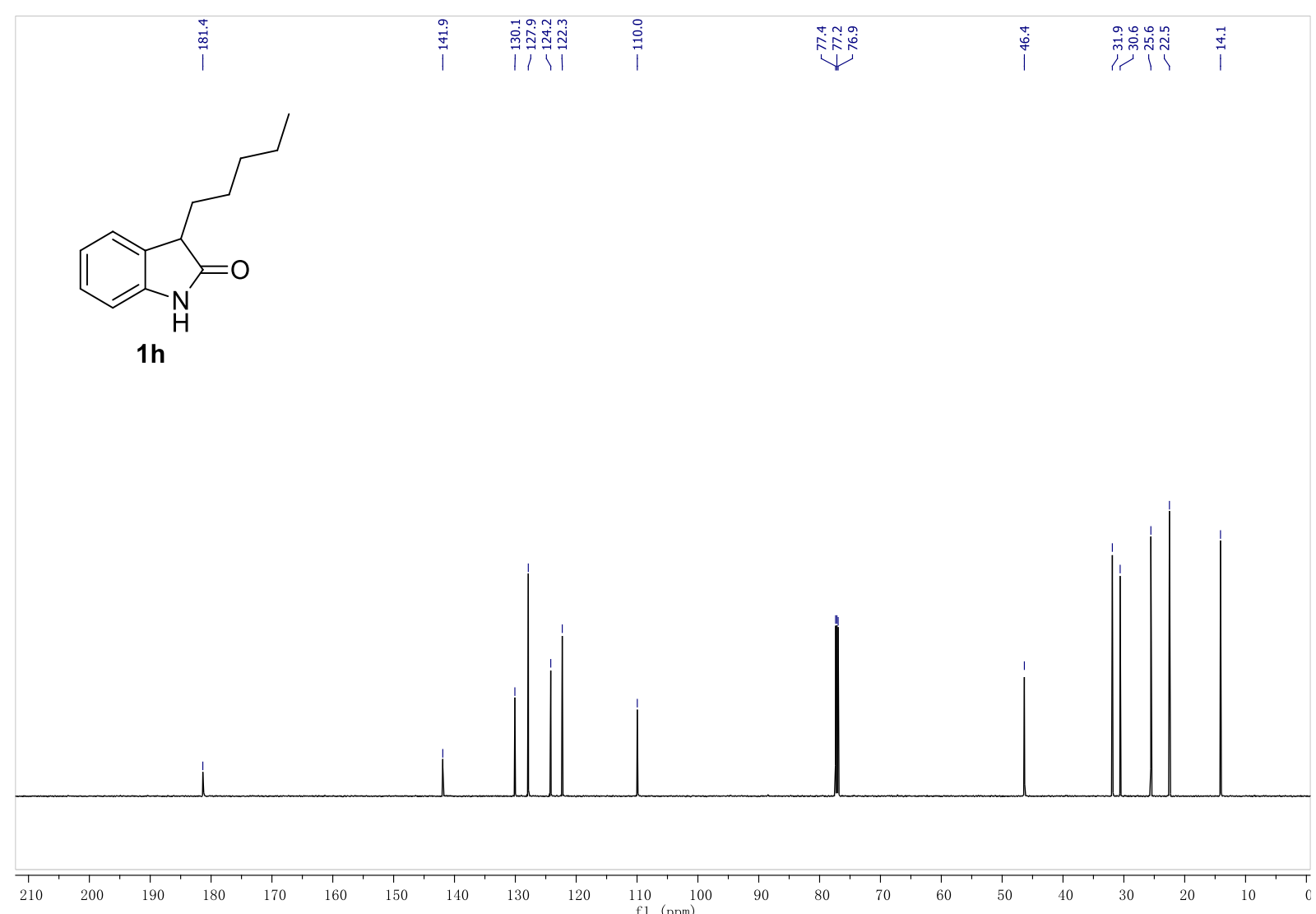

${ }^{13} \mathrm{C}\left\{{ }^{1} \mathrm{H}\right\} \mathrm{NMR}$ in $\mathrm{CDCl}_{3}$ at $150 \mathrm{MHz}$ 


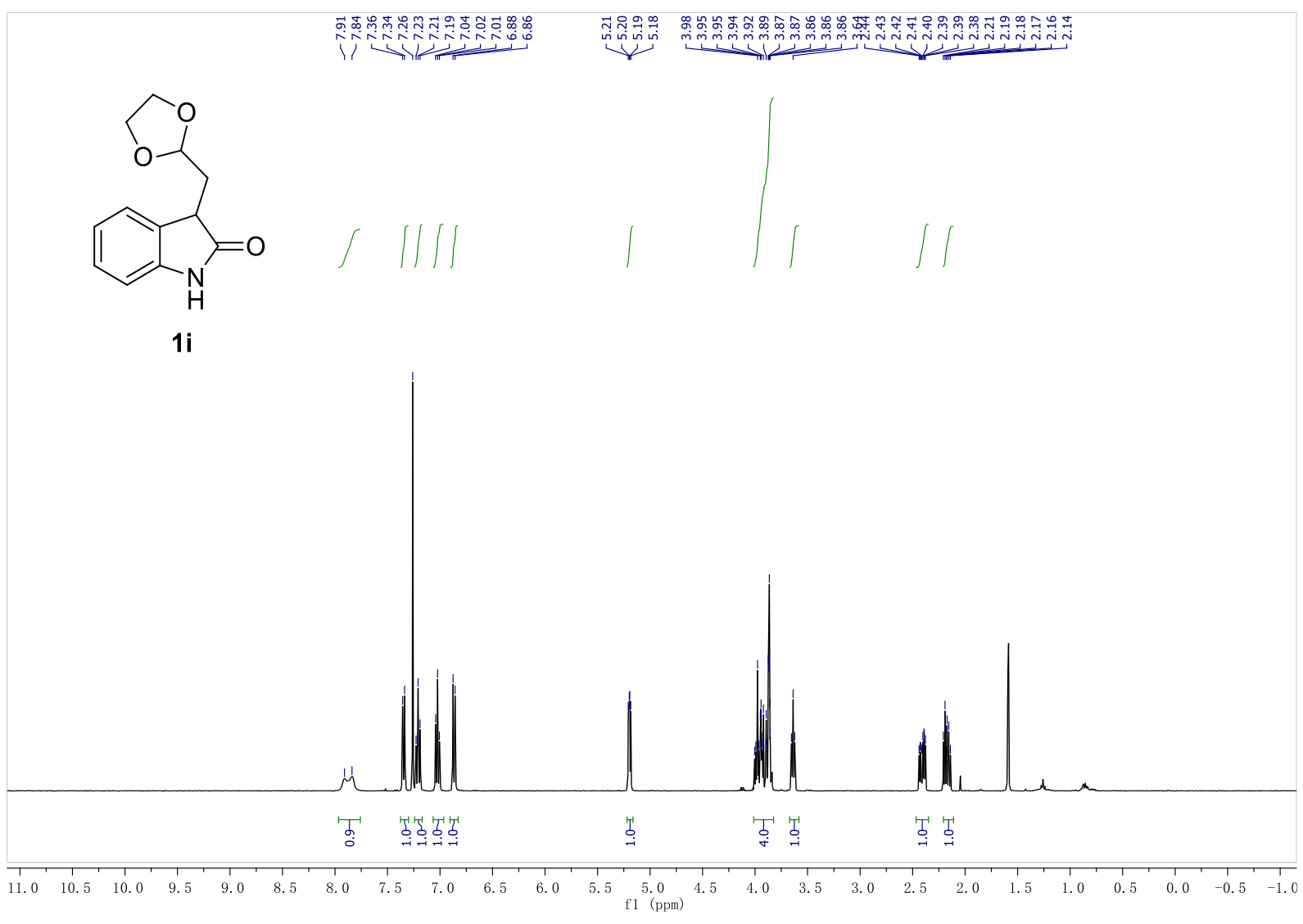

${ }^{1} \mathrm{H} \mathrm{NMR}$ in $\mathrm{CDCl}_{3}$ at $400 \mathrm{MHz}$

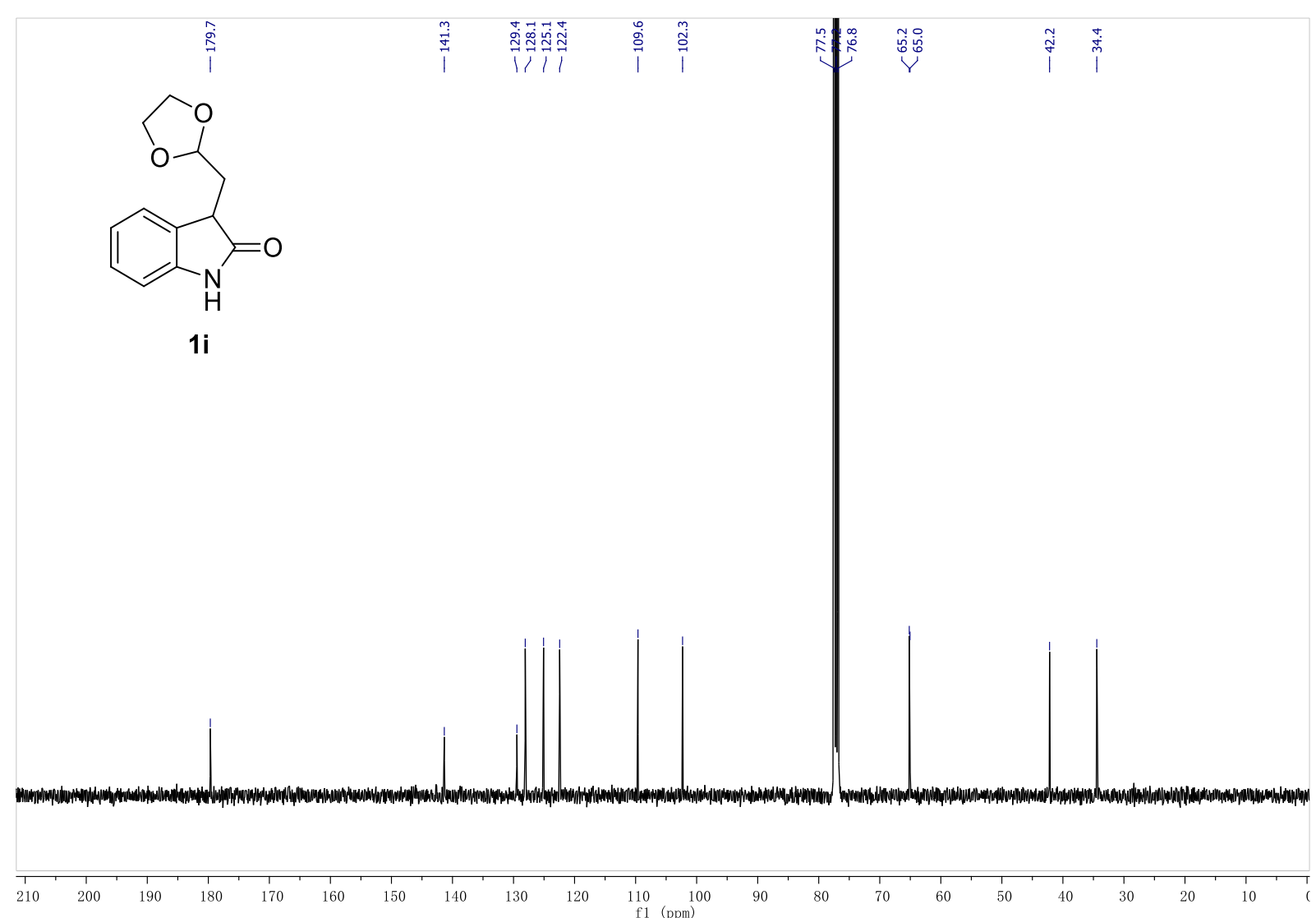

${ }^{13} \mathrm{C}\left\{{ }^{1} \mathrm{H}\right\}$ NMR in $\mathrm{CDCl}_{3}$ at $100 \mathrm{MHz}$ 


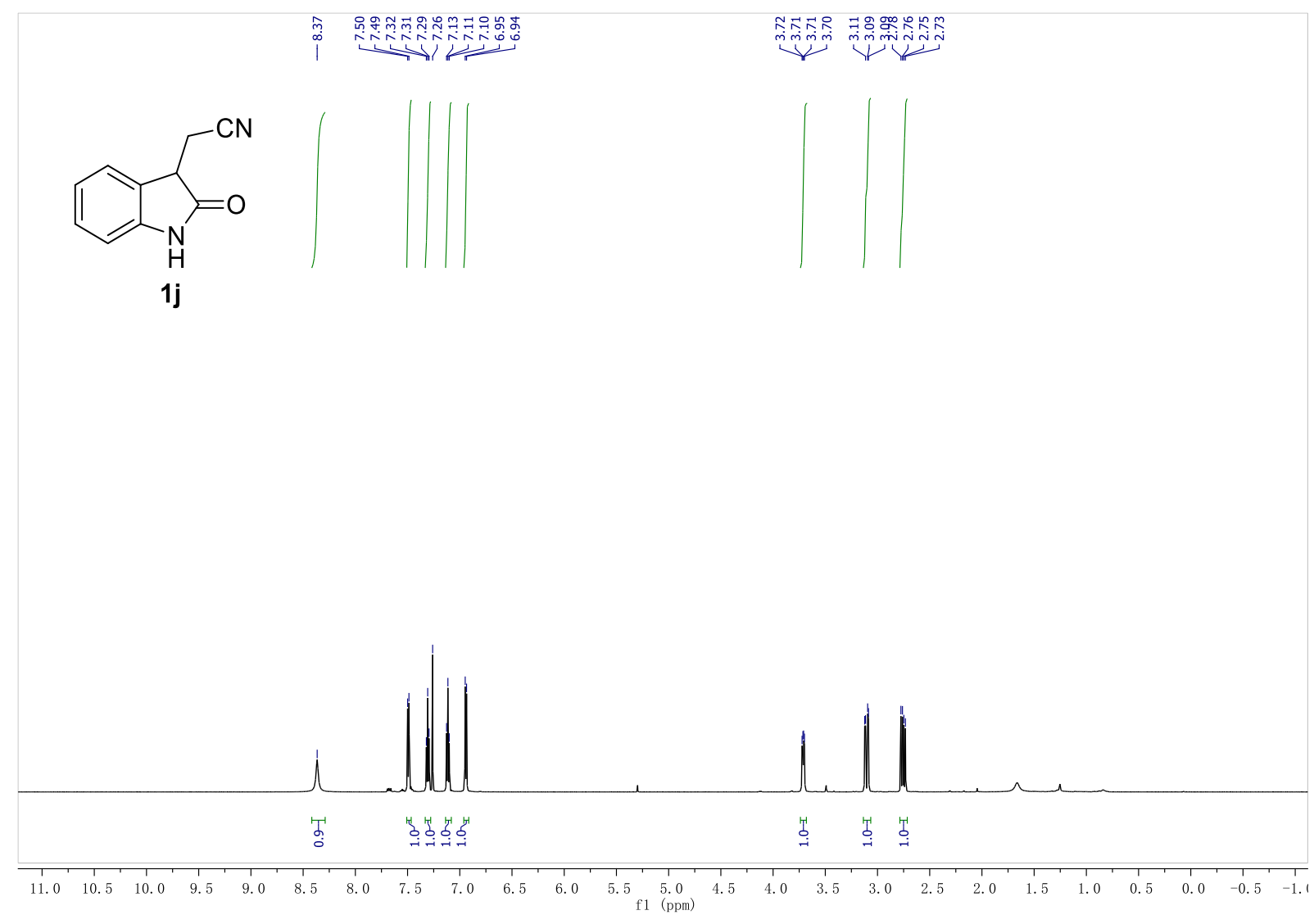

${ }^{1} \mathrm{H} \mathrm{NMR}$ in $\mathrm{CDCl}_{3}$ at $600 \mathrm{MHz}$

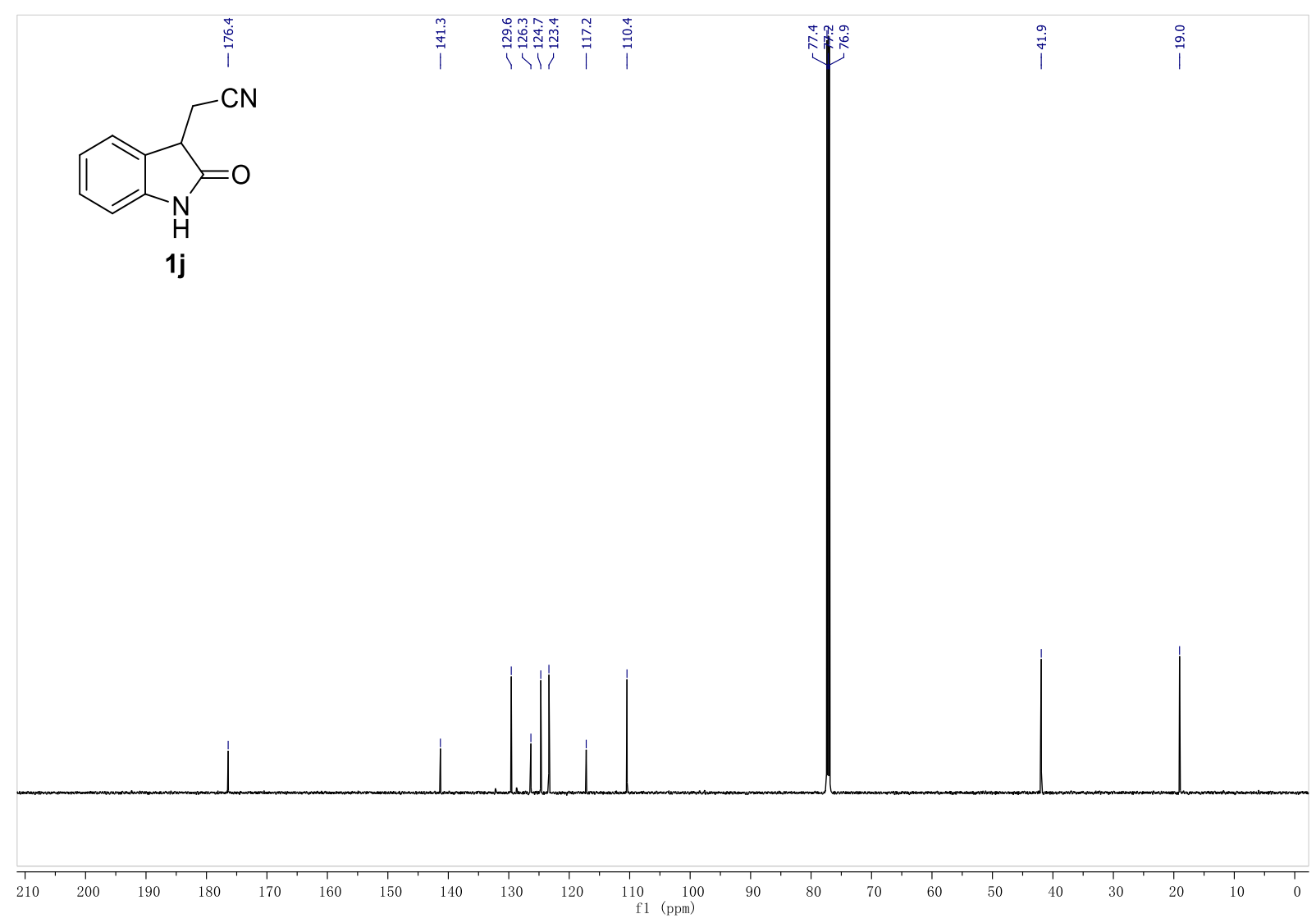

${ }^{13} \mathrm{C}\left\{{ }^{1} \mathrm{H}\right\} \mathrm{NMR}$ in $\mathrm{CDCl}_{3}$ at $150 \mathrm{MHz}$ 


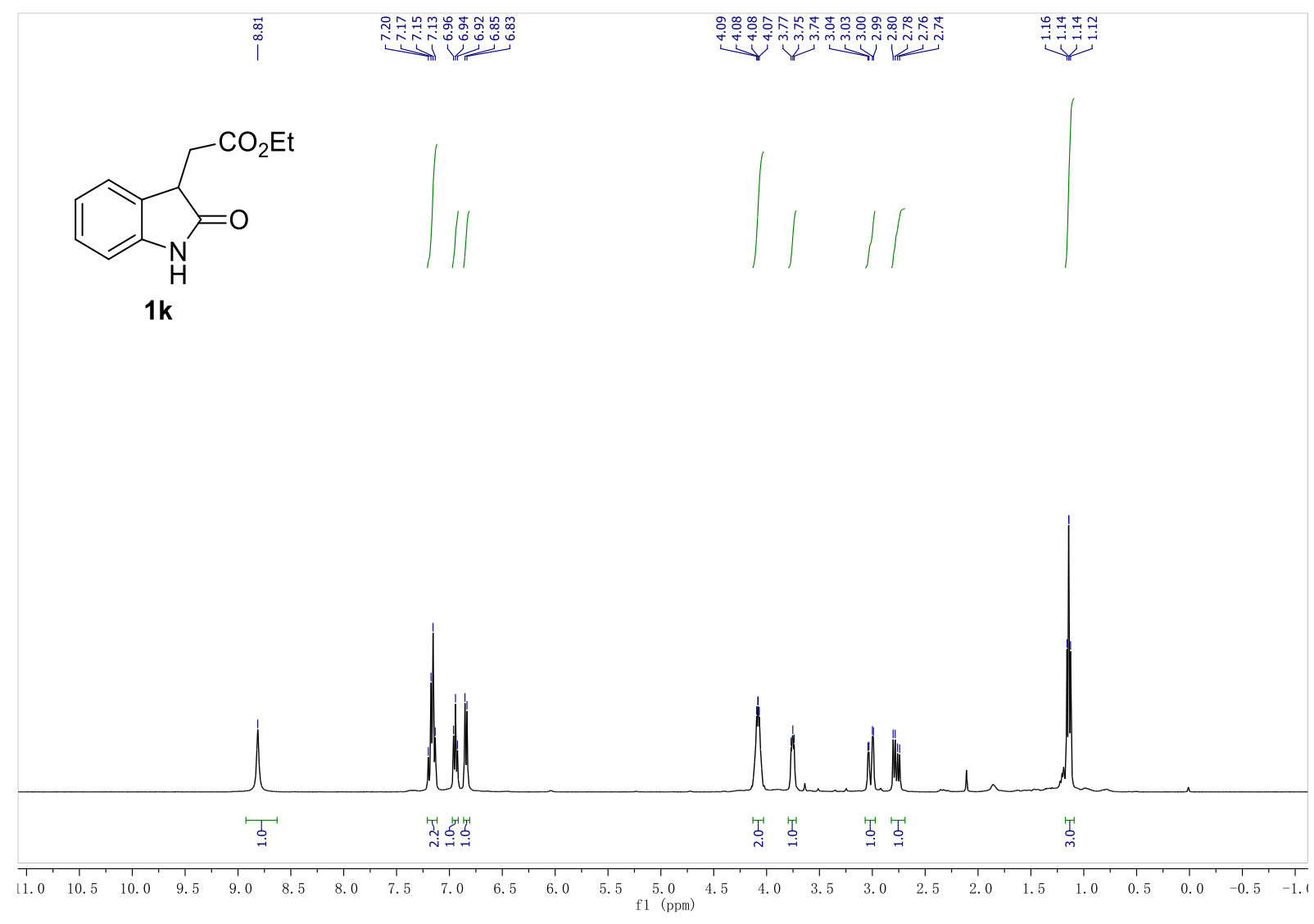

${ }^{1} \mathrm{H} \mathrm{NMR}$ in $\mathrm{CDCl}_{3}$ at $400 \mathrm{MHz}$

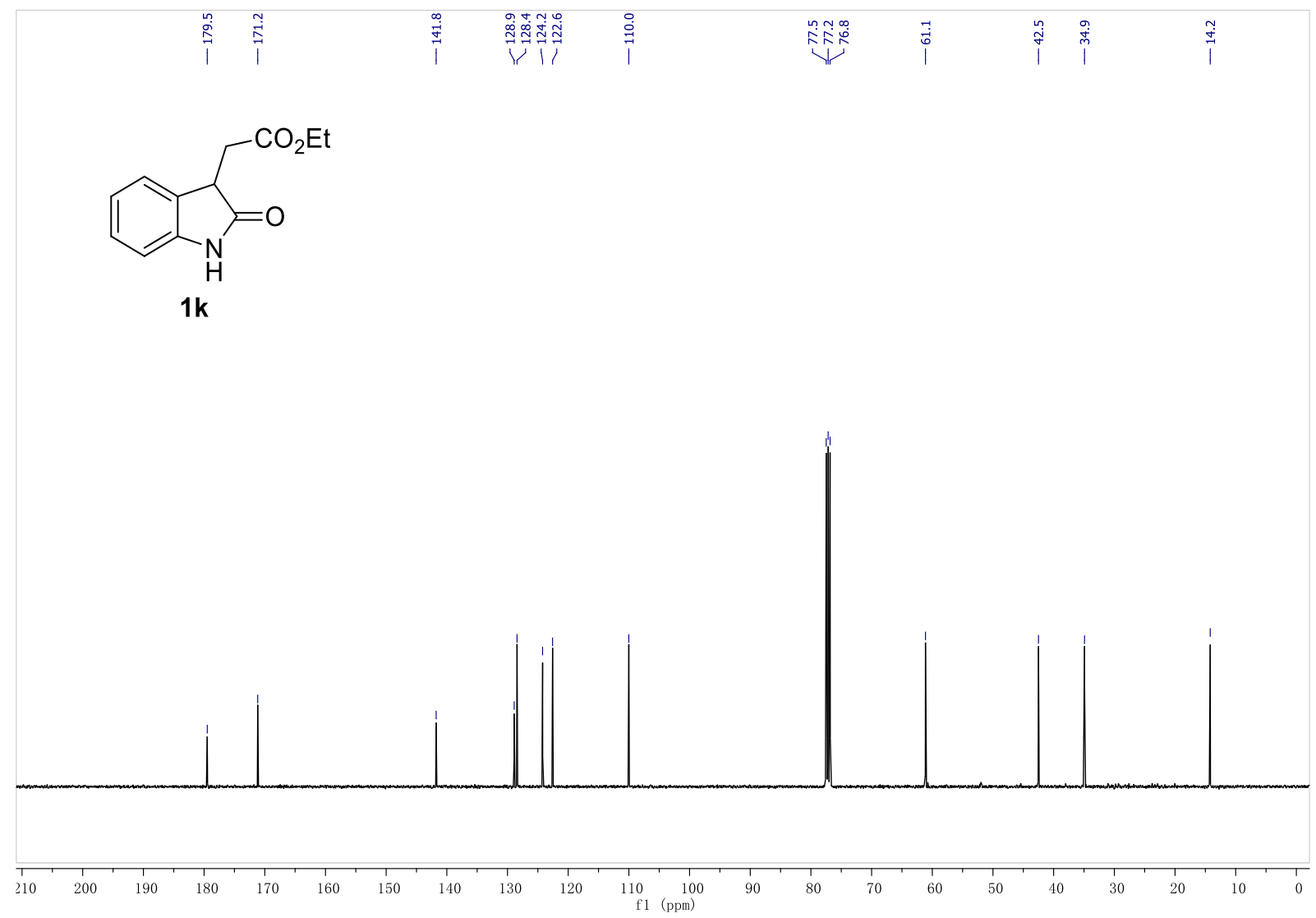

${ }^{13} \mathrm{C}\left\{{ }^{1} \mathrm{H}\right\} \mathrm{NMR}$ in $\mathrm{CDCl}_{3}$ at $100 \mathrm{MHz}$ 


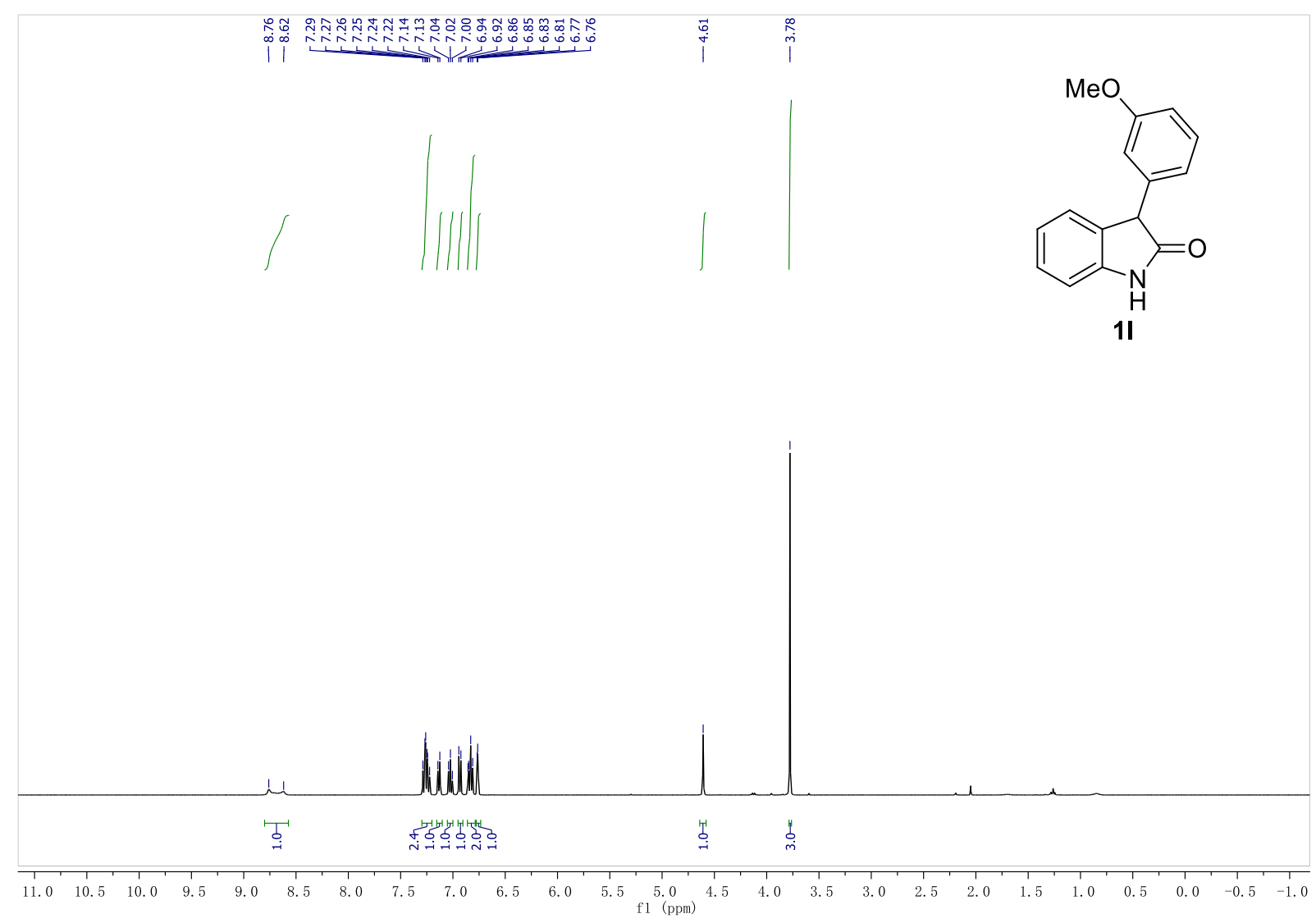

${ }^{1} \mathrm{H} \mathrm{NMR}$ in $\mathrm{CDCl}_{3}$ at $400 \mathrm{MHz}$
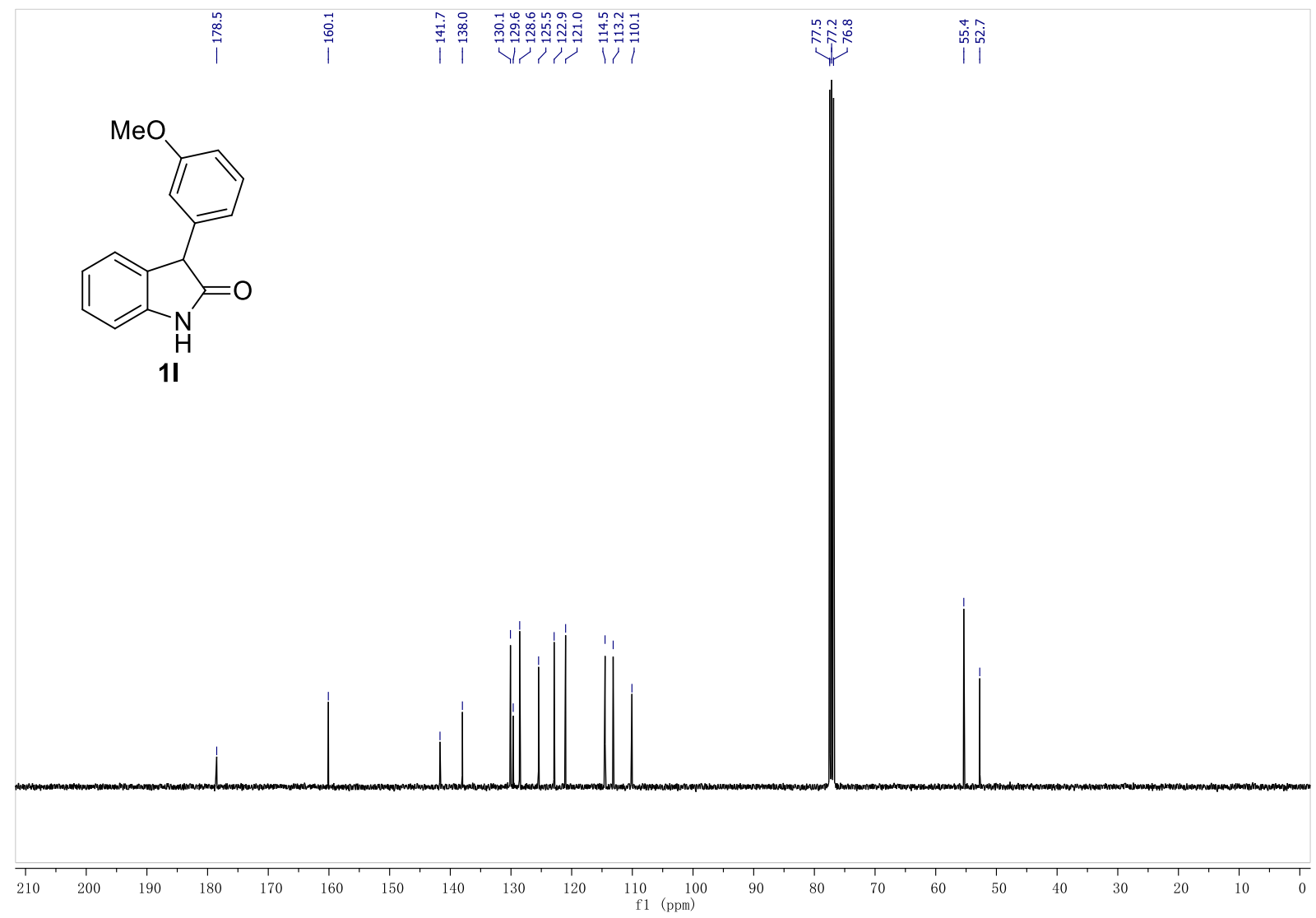

${ }^{13} \mathrm{C}\left\{{ }^{1} \mathrm{H}\right\} \mathrm{NMR}$ in $\mathrm{CDCl}_{3}$ at $100 \mathrm{MHz}$ 


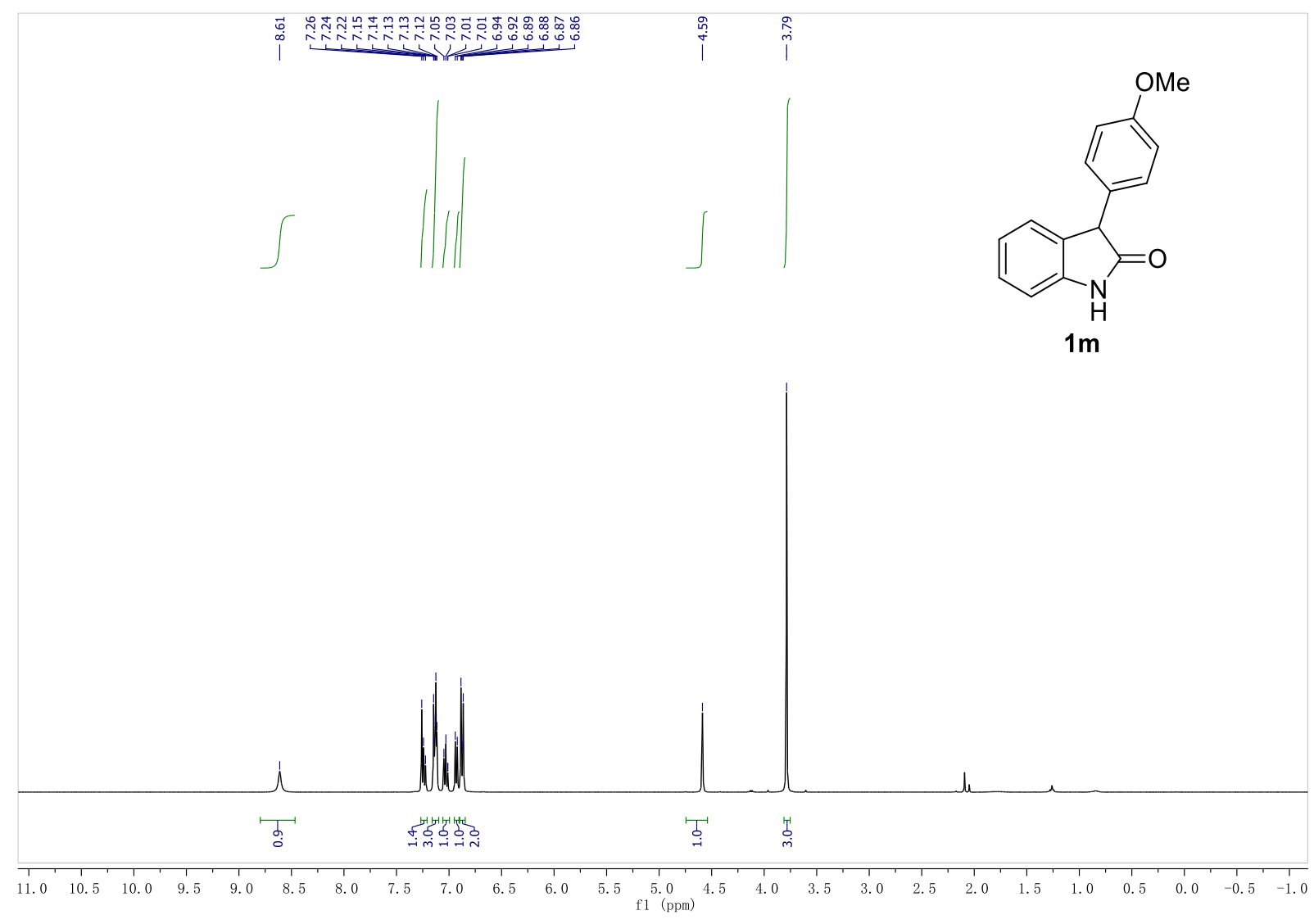

${ }^{1} \mathrm{H} \mathrm{NMR}$ in $\mathrm{CDCl}_{3}$ at $400 \mathrm{MHz}$

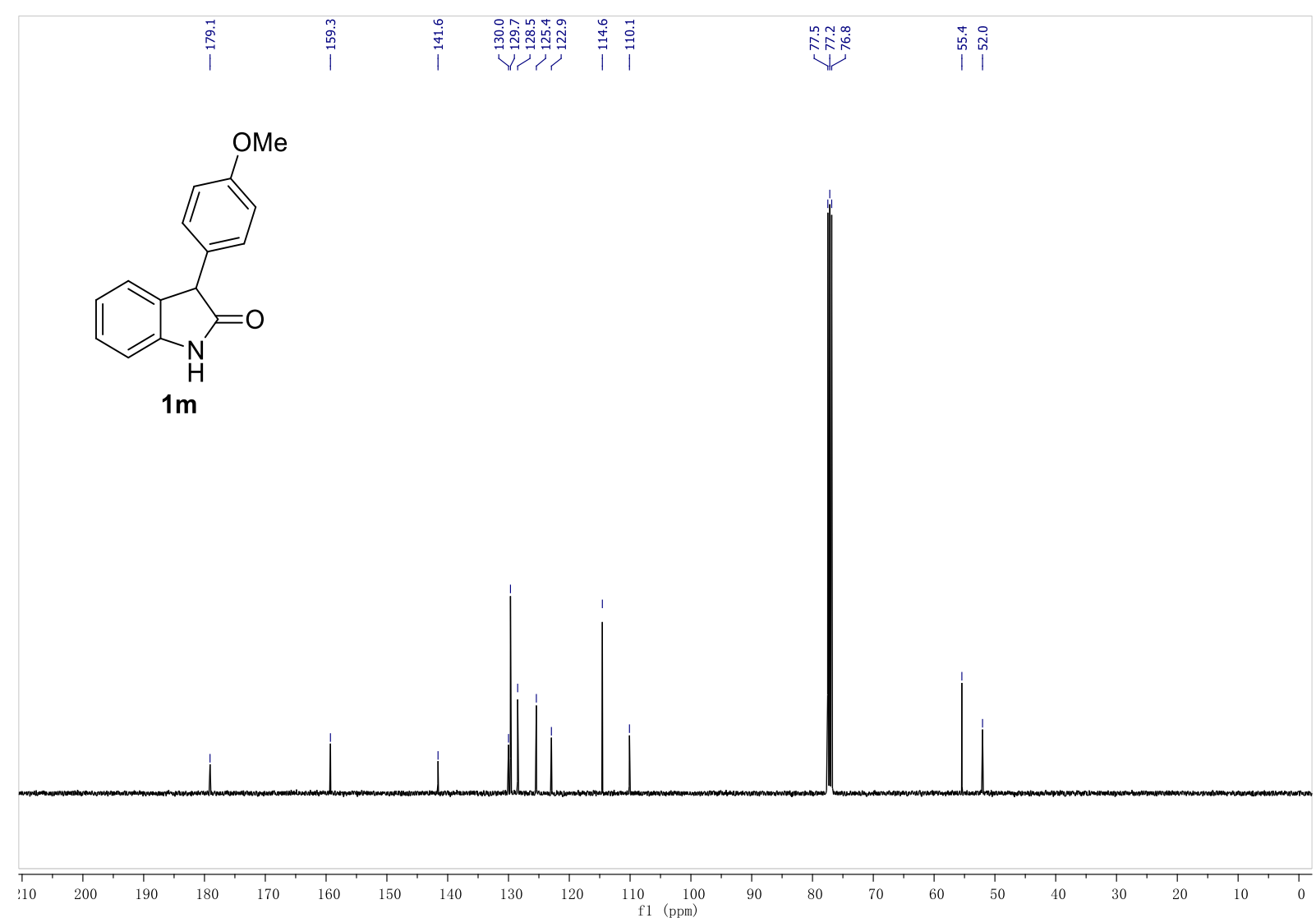

${ }^{13} \mathrm{C}\left\{{ }^{1} \mathrm{H}\right\} \mathrm{NMR}$ in $\mathrm{CDCl}_{3}$ at $100 \mathrm{MHz}$ 


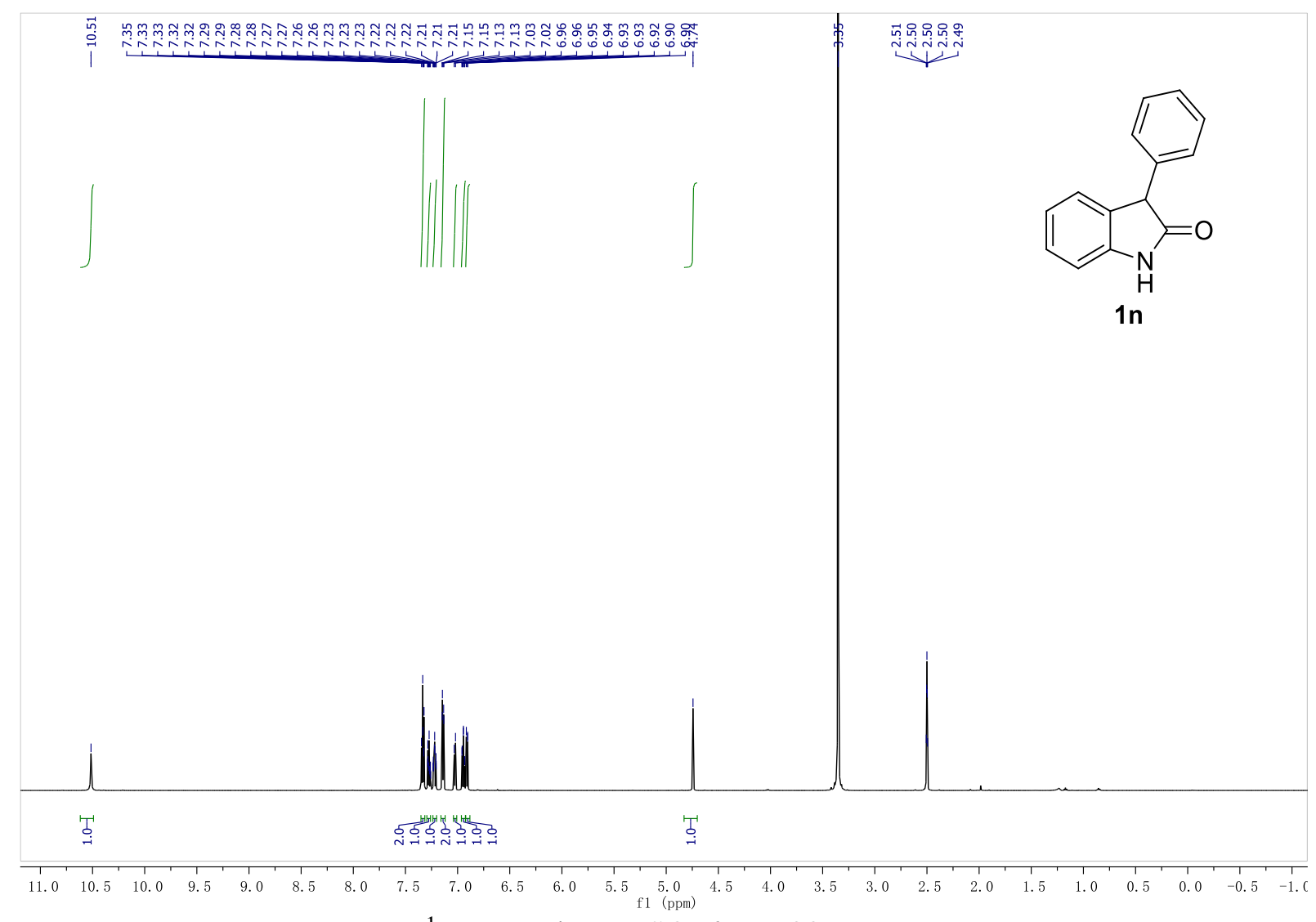

${ }^{1} \mathrm{H}$ NMR in DMSO- $d_{6}$ at $600 \mathrm{MHz}$

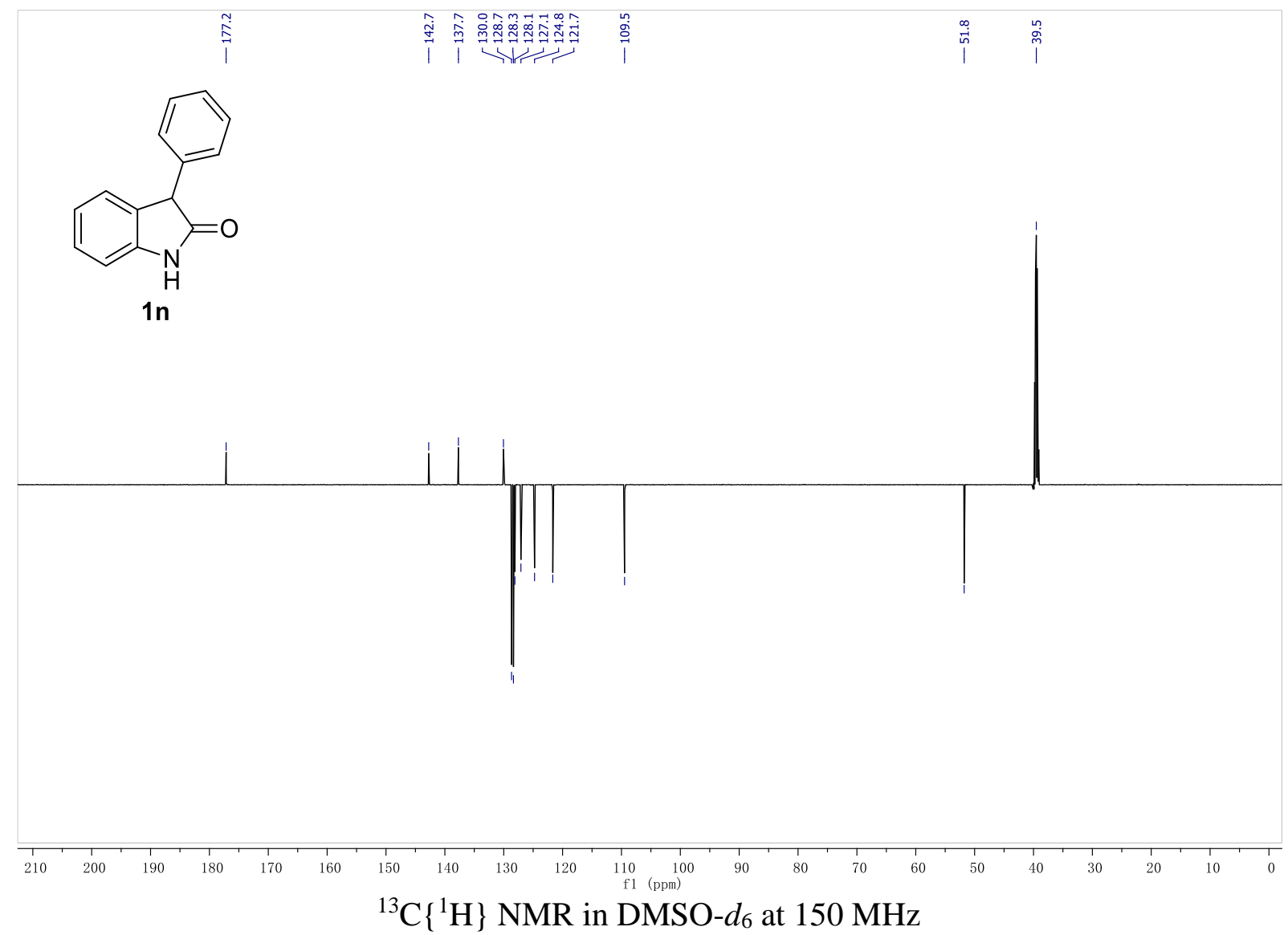




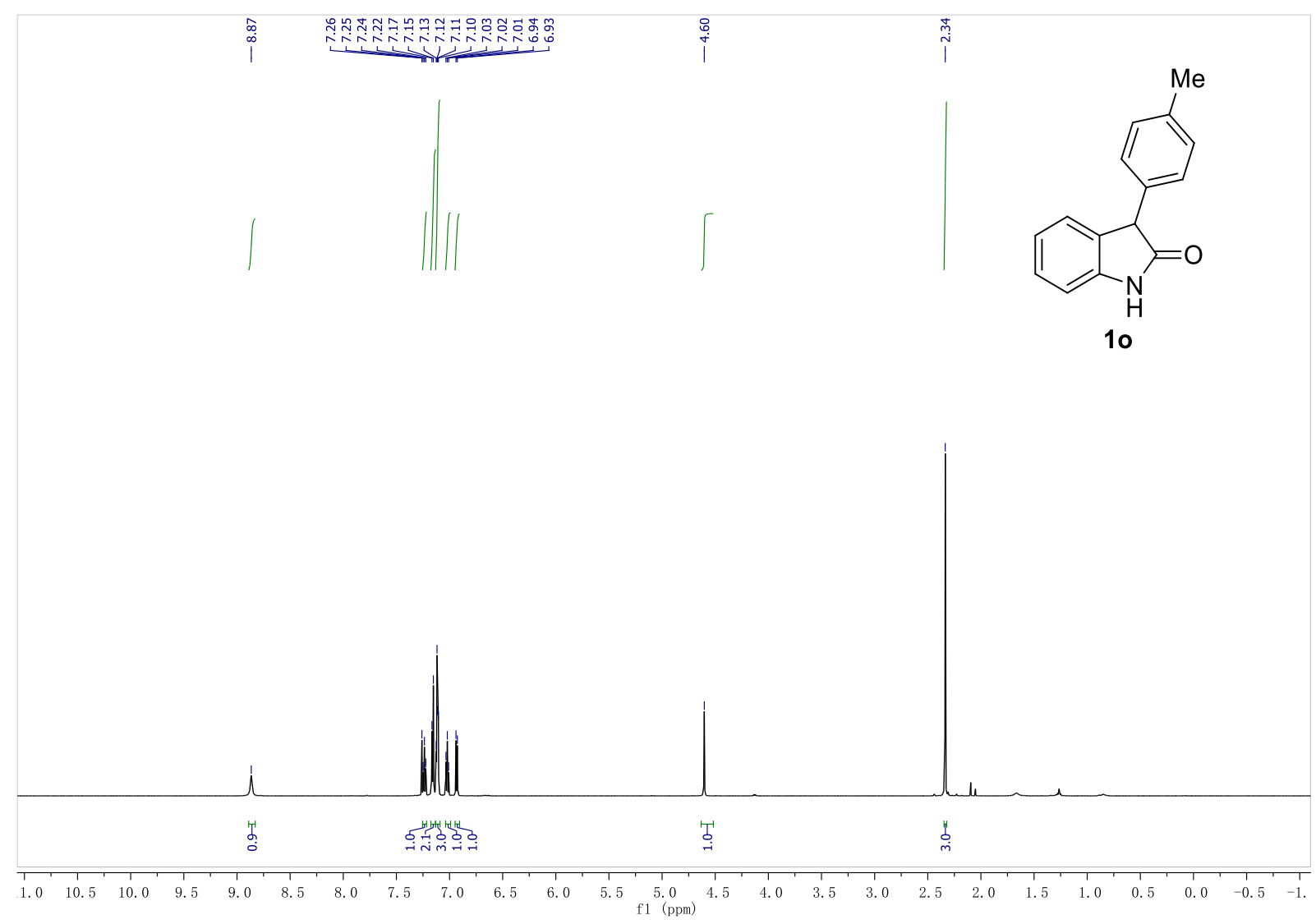

${ }^{1} \mathrm{H} \mathrm{NMR}$ in $\mathrm{CDCl}_{3}$ at $600 \mathrm{MHz}$

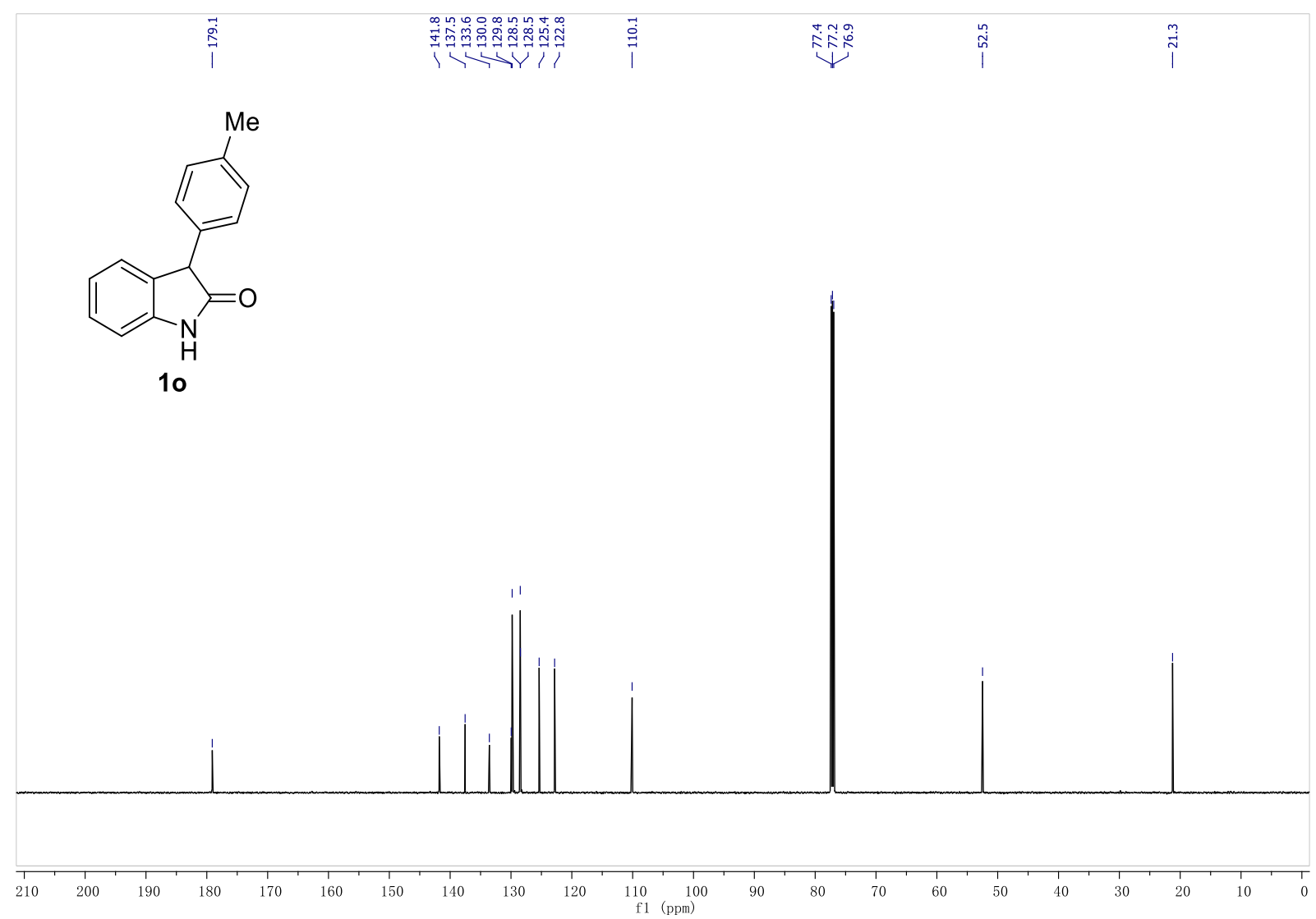

${ }^{13} \mathrm{C}\left\{{ }^{1} \mathrm{H}\right\} \mathrm{NMR}$ in $\mathrm{CDCl}_{3}$ at $150 \mathrm{MHz}$ 


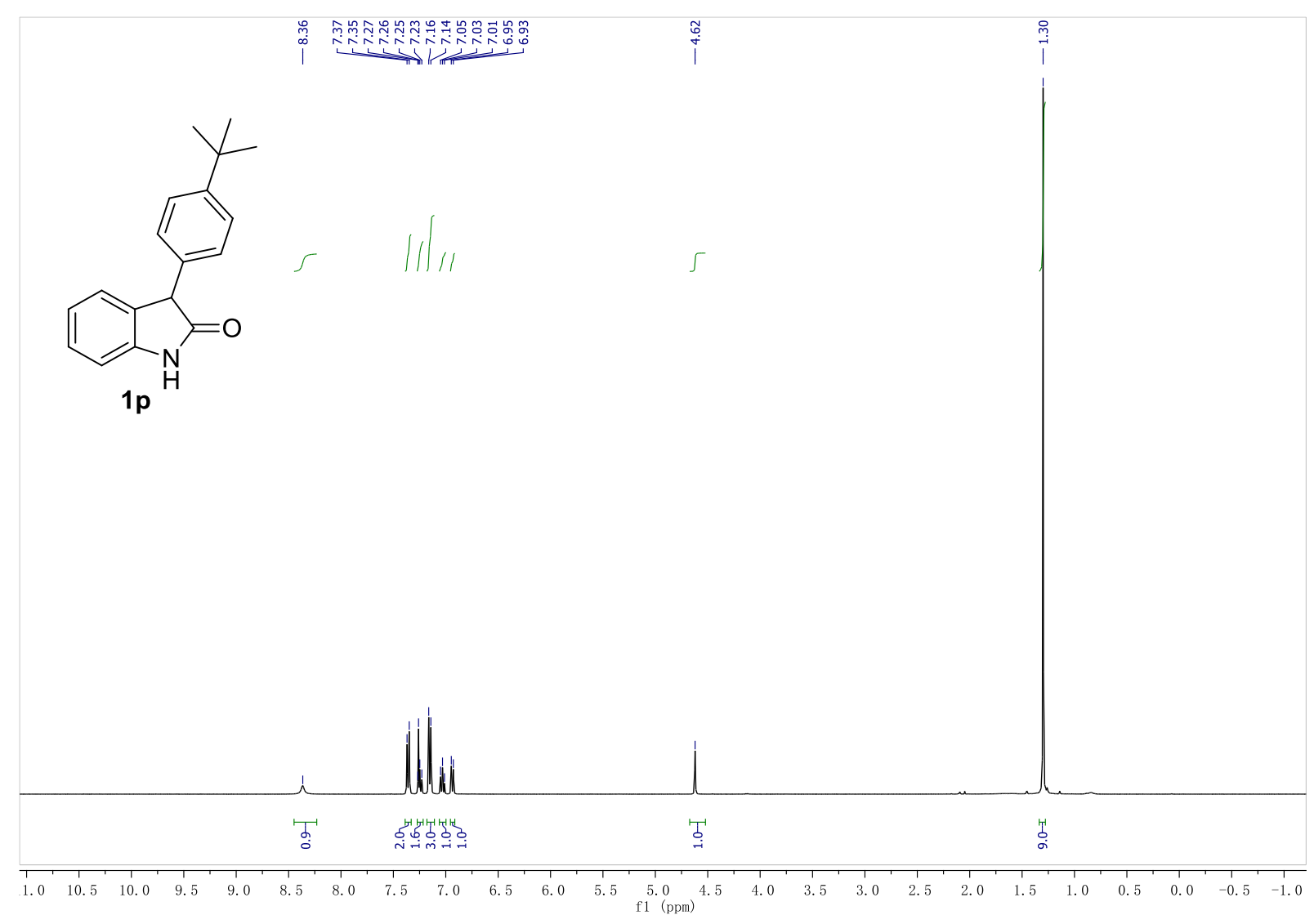

${ }^{1} \mathrm{H} \mathrm{NMR}$ in $\mathrm{CDCl}_{3}$ at $600 \mathrm{MHz}$

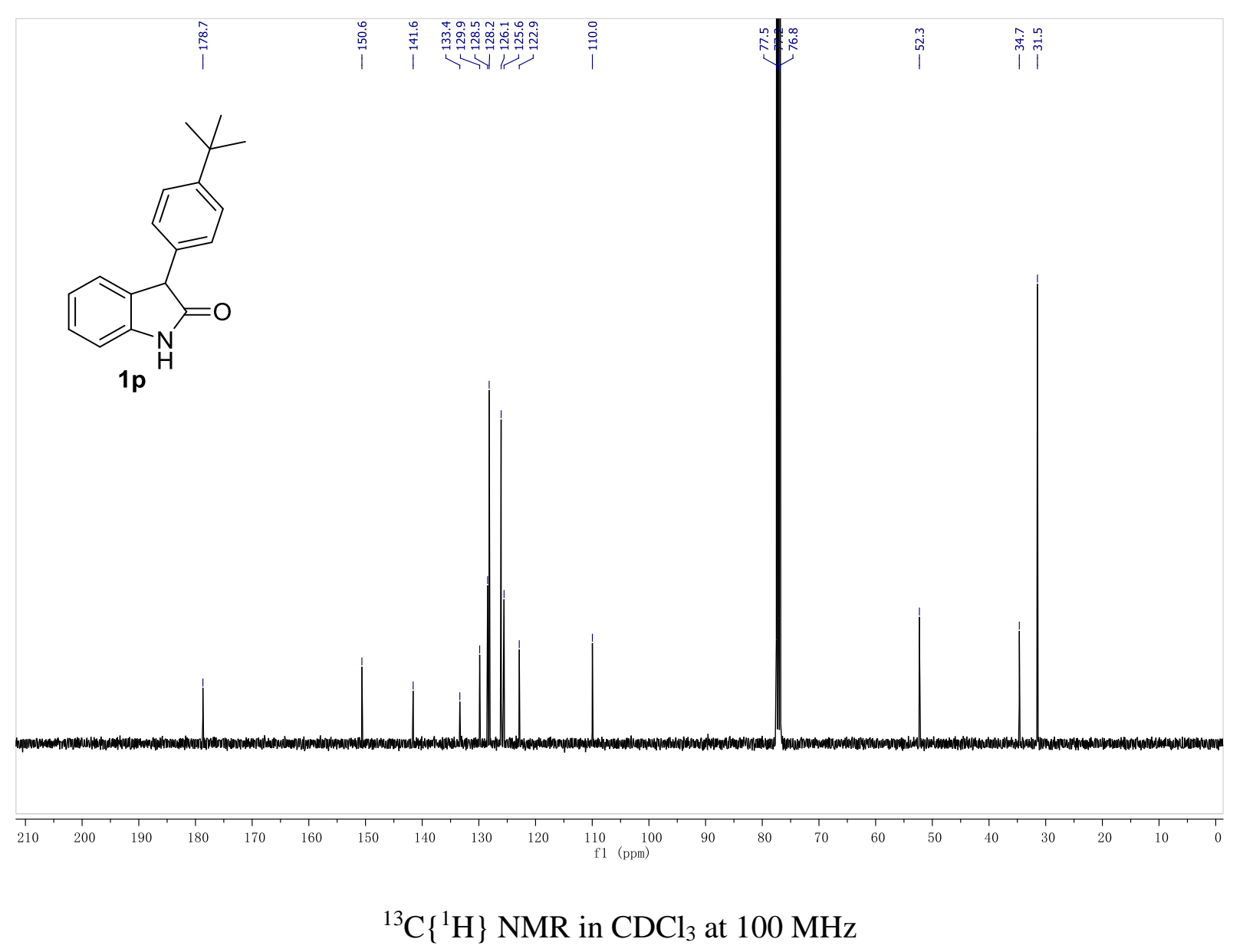




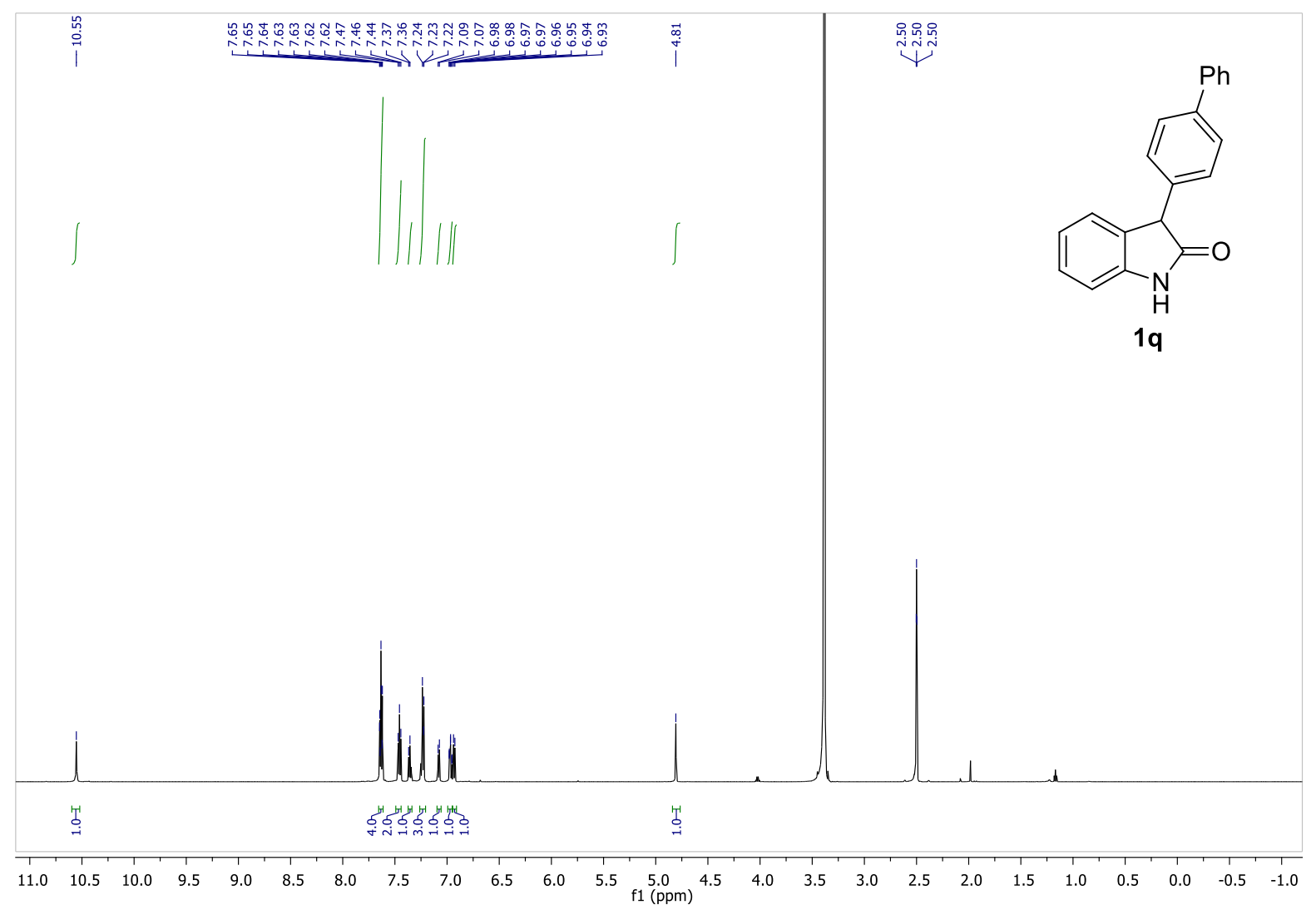

${ }^{1} \mathrm{H}$ NMR in DMSO- $d_{6}$ at $600 \mathrm{MHz}$

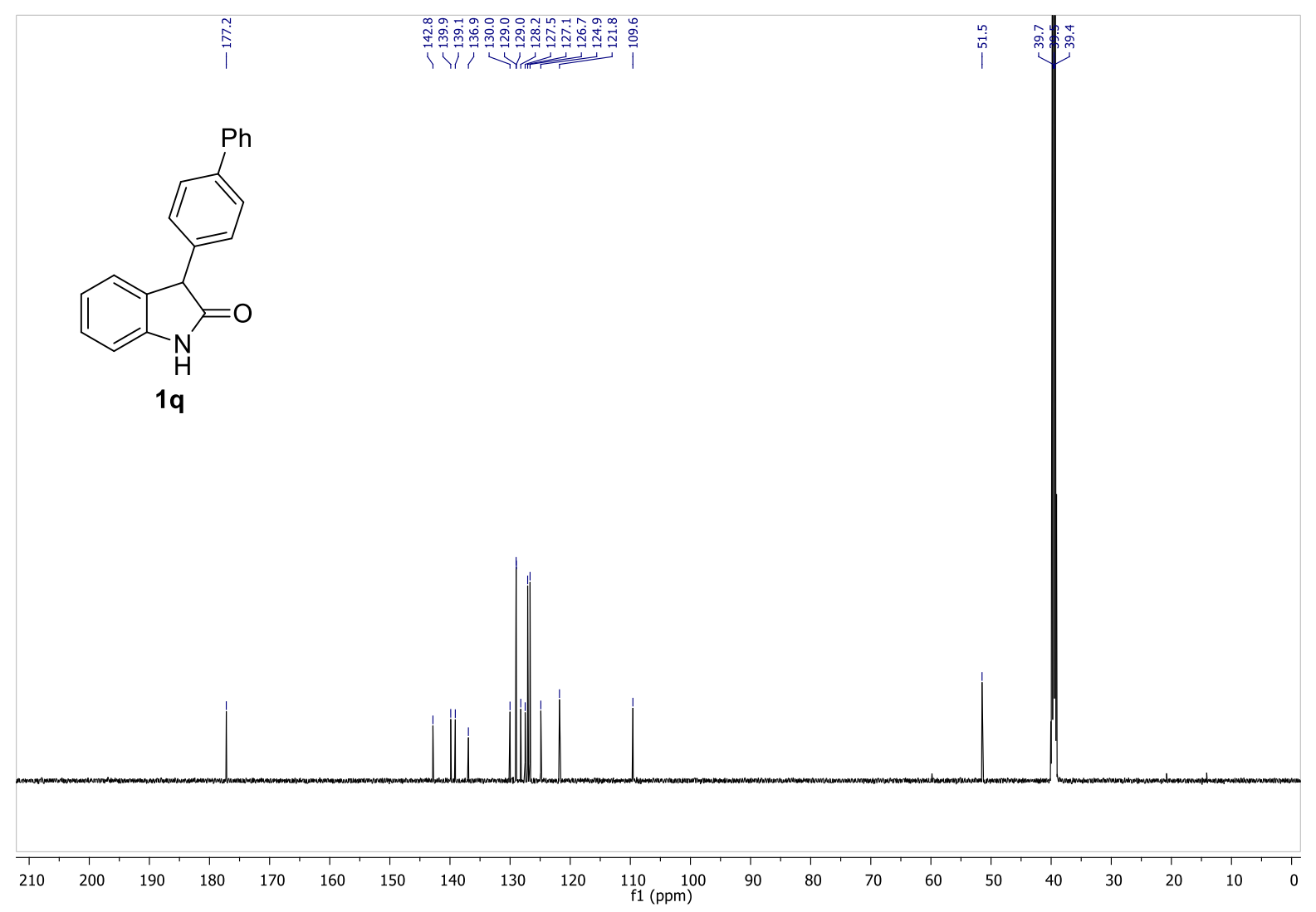

${ }^{13} \mathrm{C}\left\{{ }^{1} \mathrm{H}\right\}$ NMR in DMSO- $d_{6}$ at $150 \mathrm{MHz}$ 


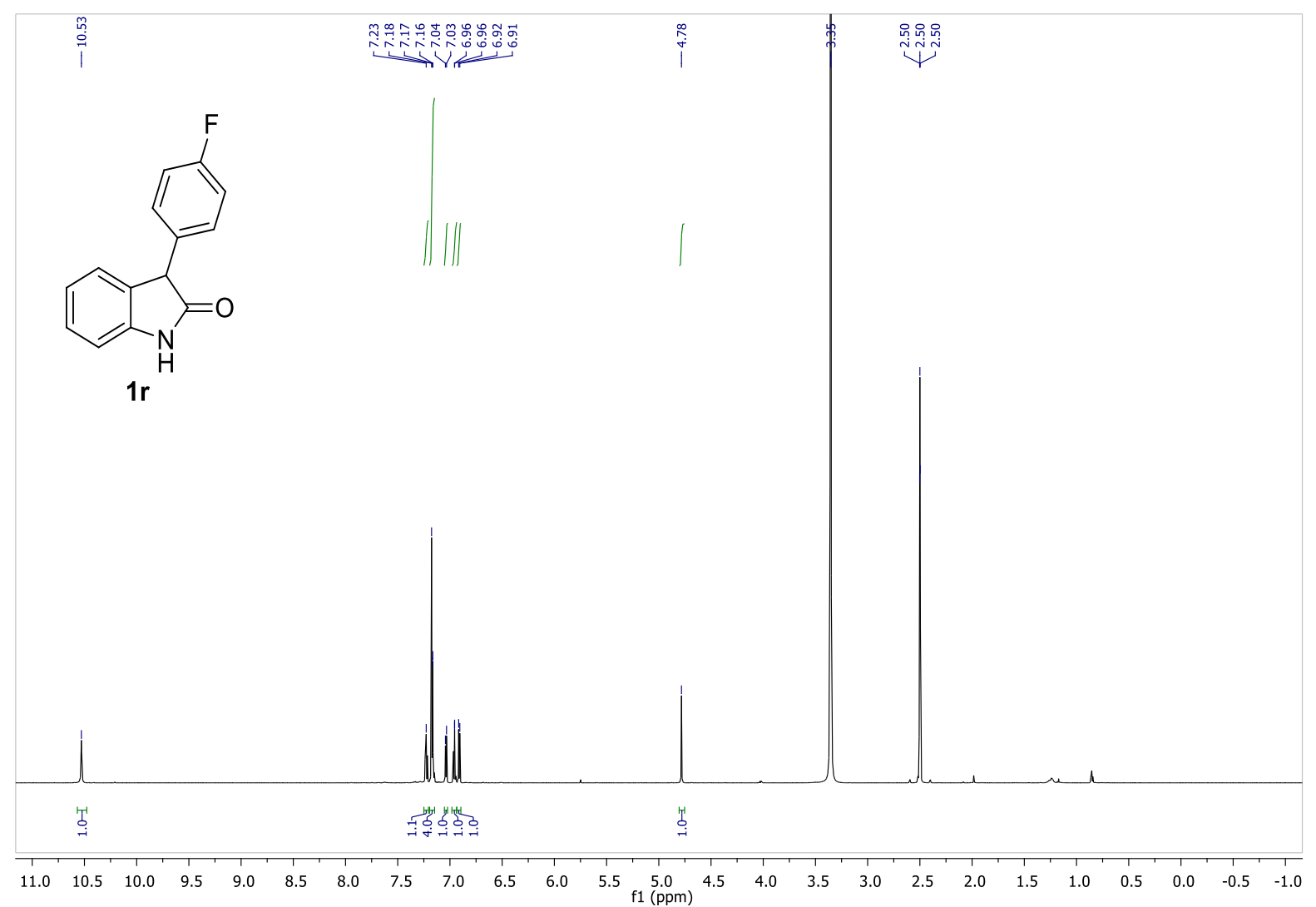

${ }^{1} \mathrm{H}$ NMR in DMSO- $d_{6}$ at $700 \mathrm{MHz}$

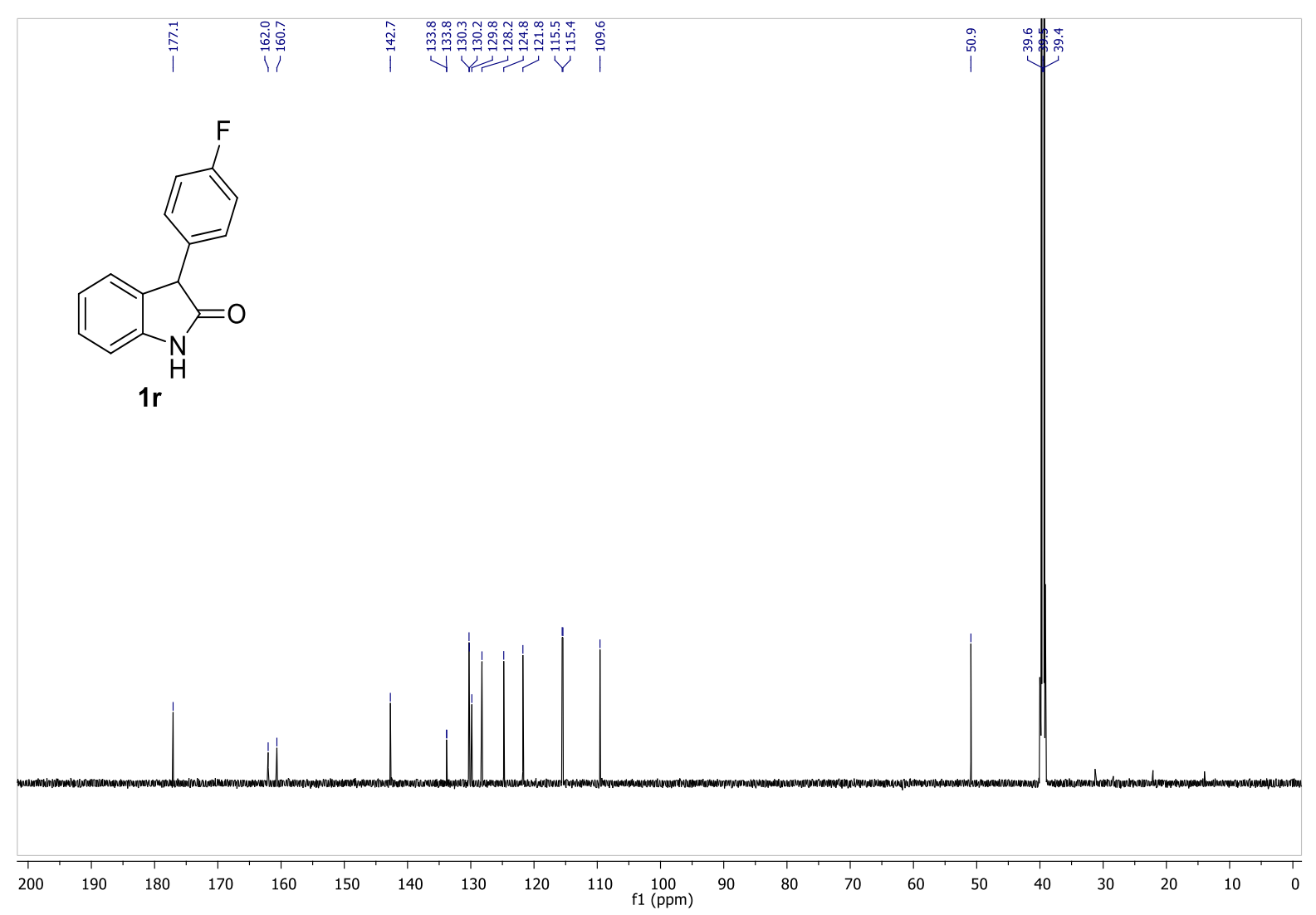

${ }^{13} \mathrm{C}\left\{{ }^{1} \mathrm{H}\right\}$ NMR in DMSO- $d_{6}$ at $176 \mathrm{MHz}$ 


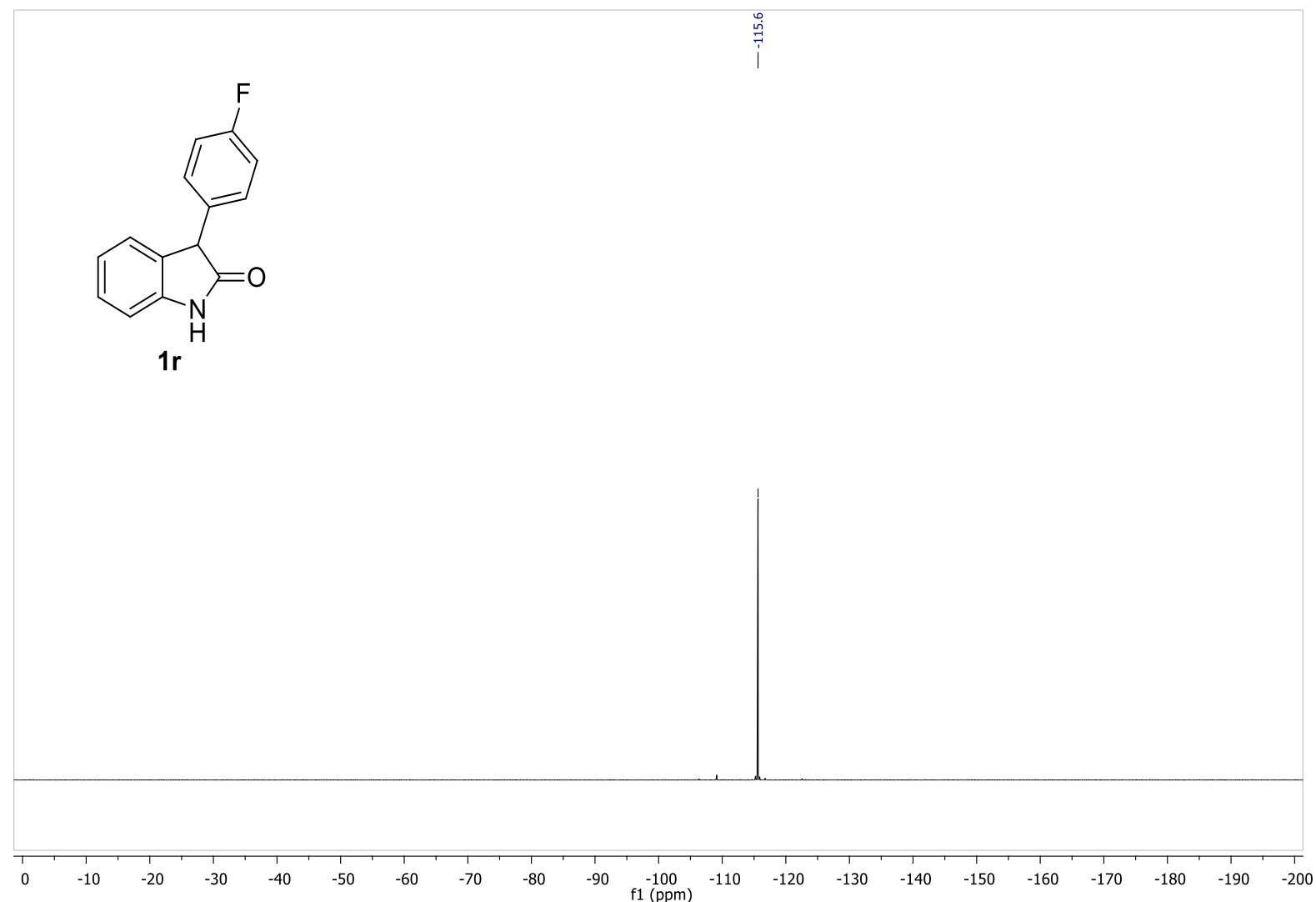

${ }^{19} \mathrm{~F}$ NMR in DMSO- $d_{6}$ at $659 \mathrm{MHz}$ 


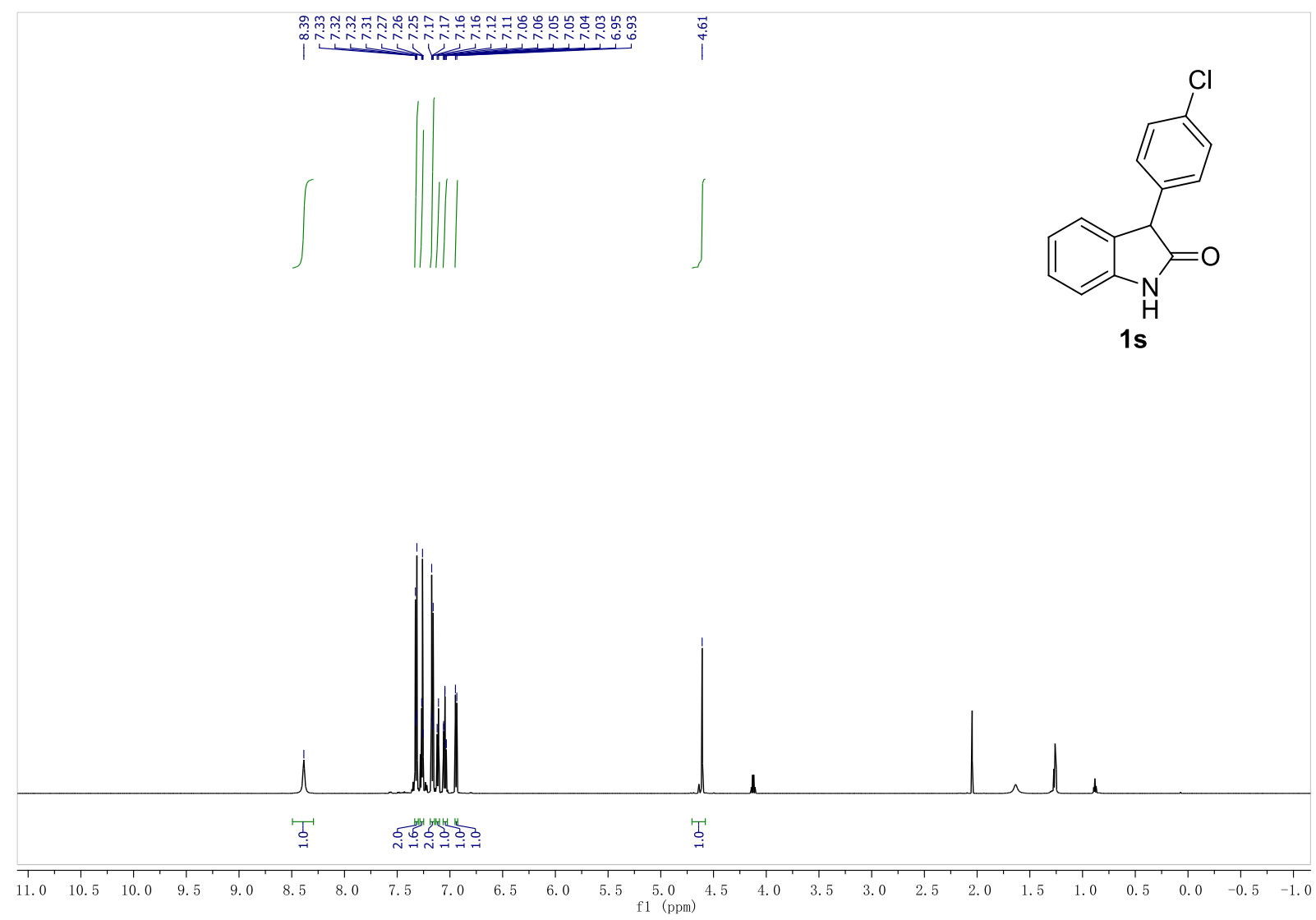

${ }^{1} \mathrm{H} \mathrm{NMR}$ in $\mathrm{CDCl}_{3}$ at $600 \mathrm{MHz}$

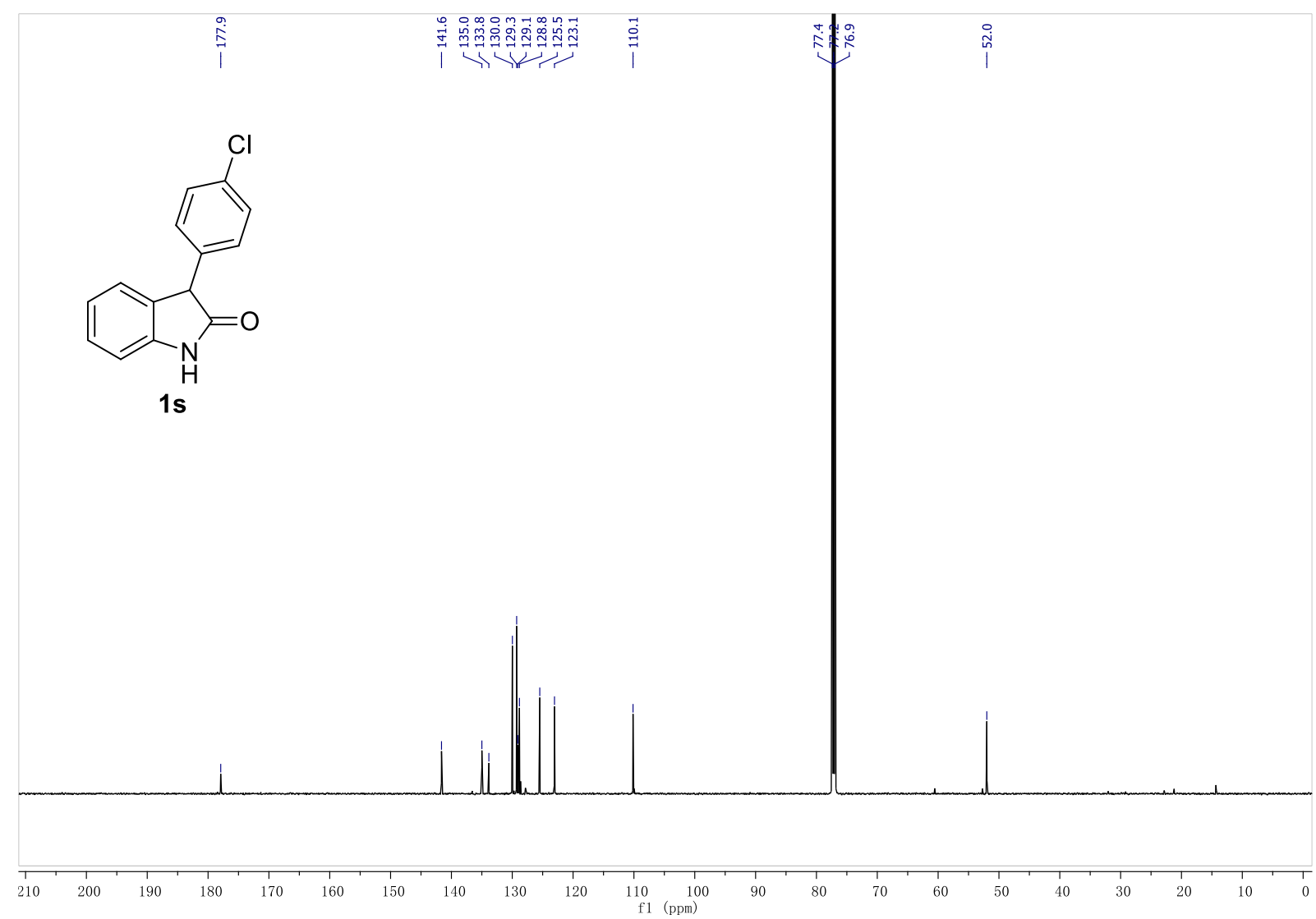

${ }^{13} \mathrm{C}\left\{{ }^{1} \mathrm{H}\right\}$ NMR in $\mathrm{CDCl}_{3}$ at $150 \mathrm{MHz}$ 


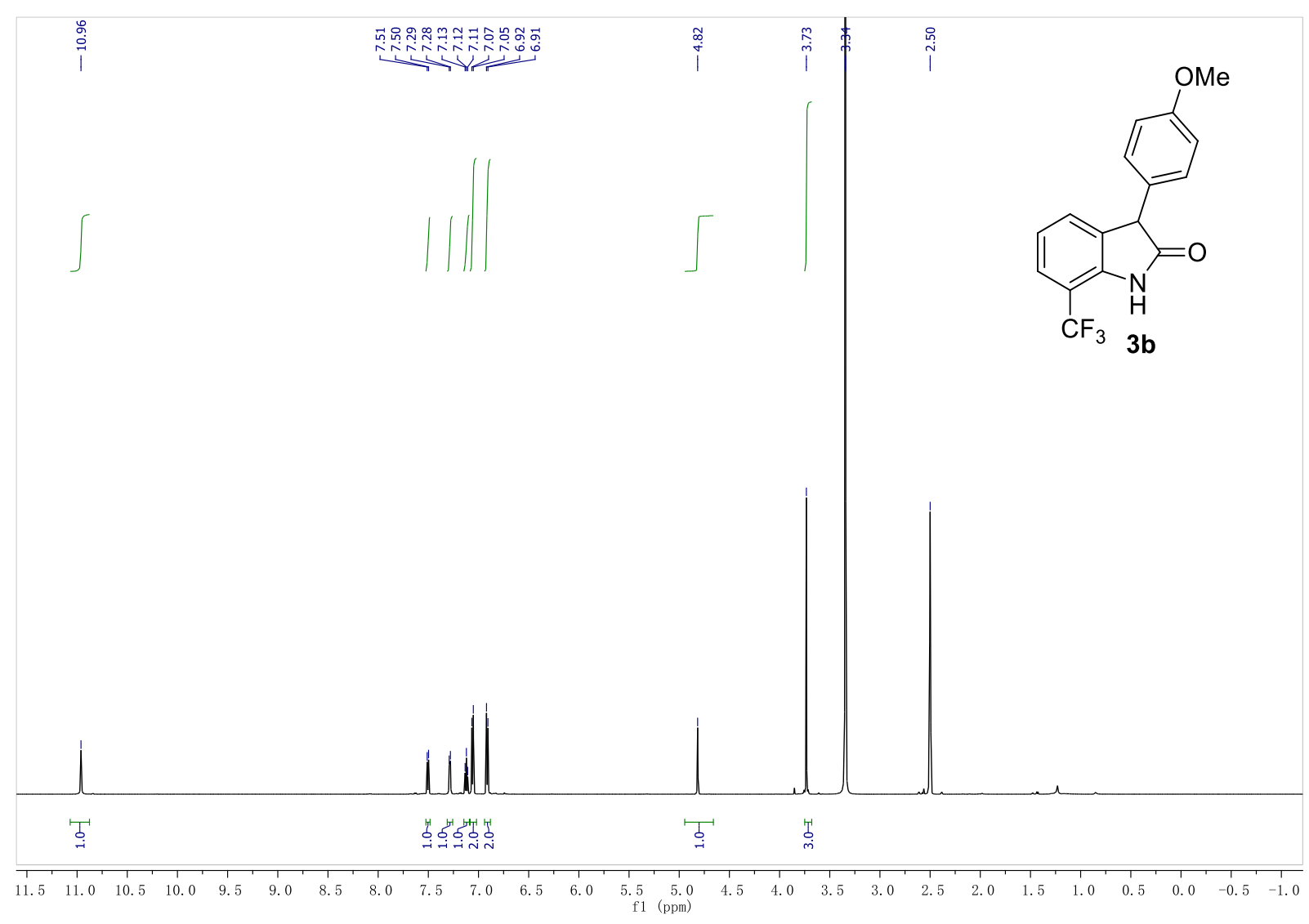

${ }^{1} \mathrm{H}$ NMR in DMSO- $d_{6}$ at $600 \mathrm{MHz}$

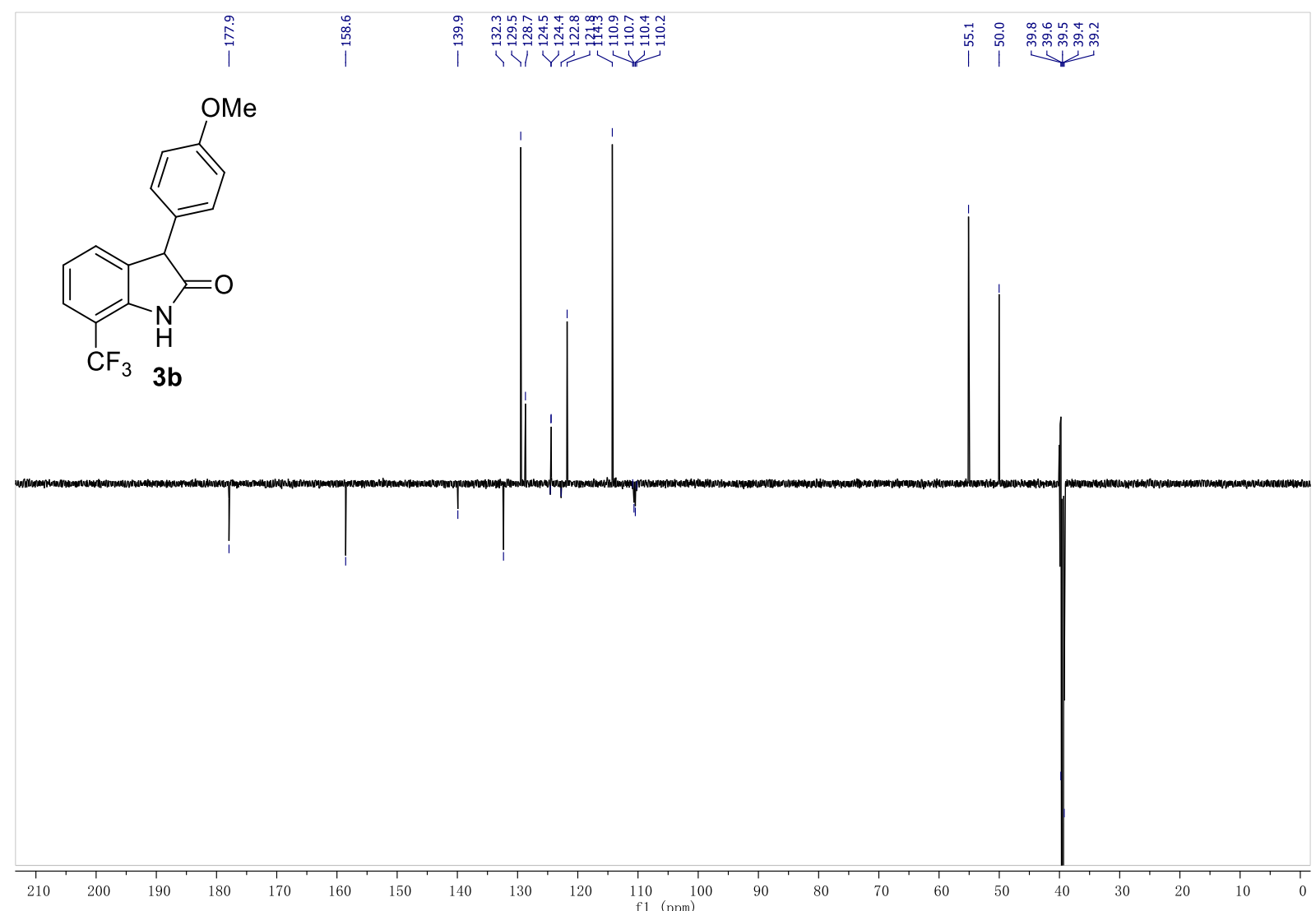

${ }^{13} \mathrm{C}\left\{{ }^{1} \mathrm{H}\right\} \mathrm{NMR}$ in DMSO- $d_{6}$ at $150 \mathrm{MHz}$ 


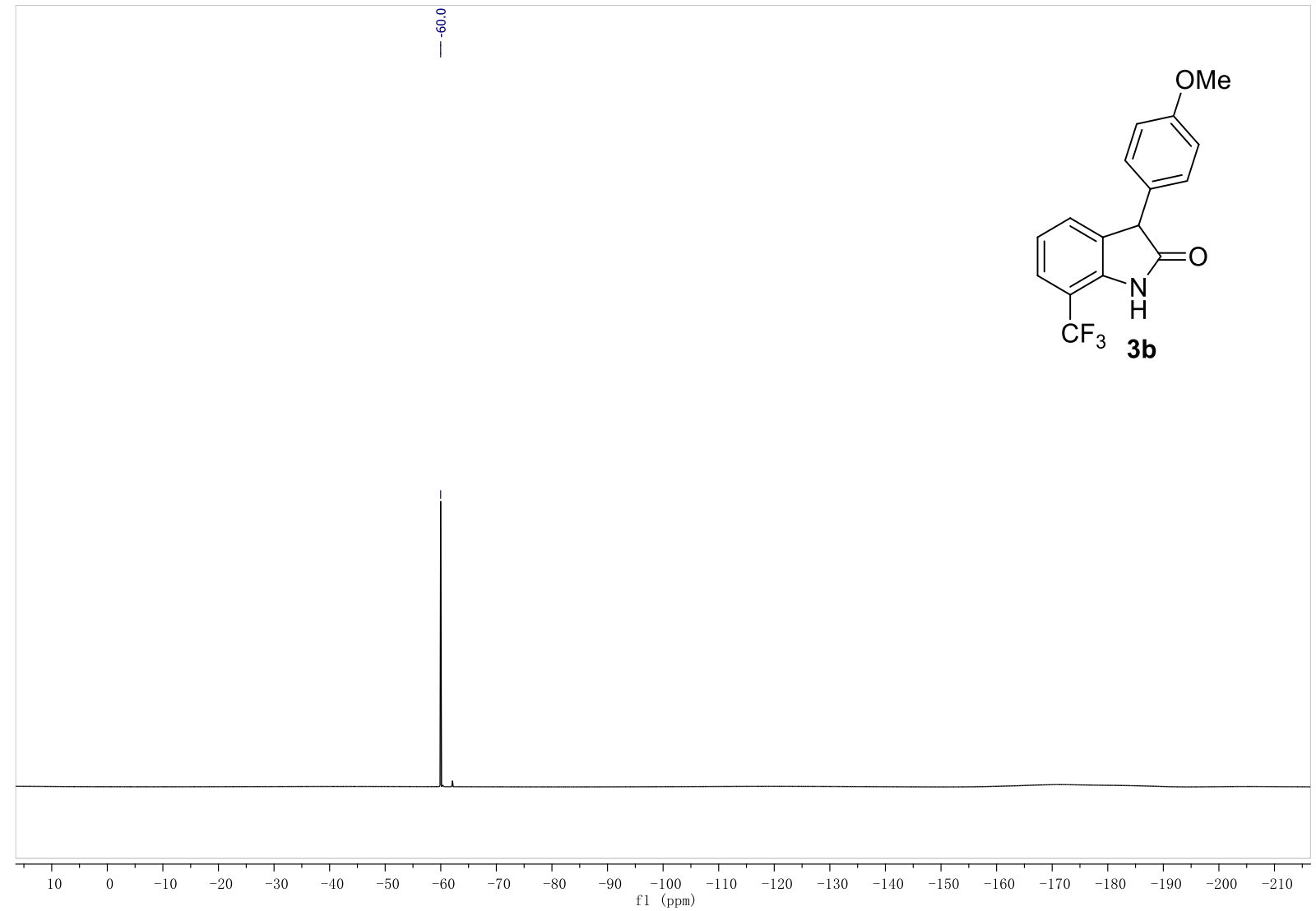

${ }^{19} \mathrm{~F}$ NMR in DMSO- $d_{6}$ at $565 \mathrm{MHz}$ 


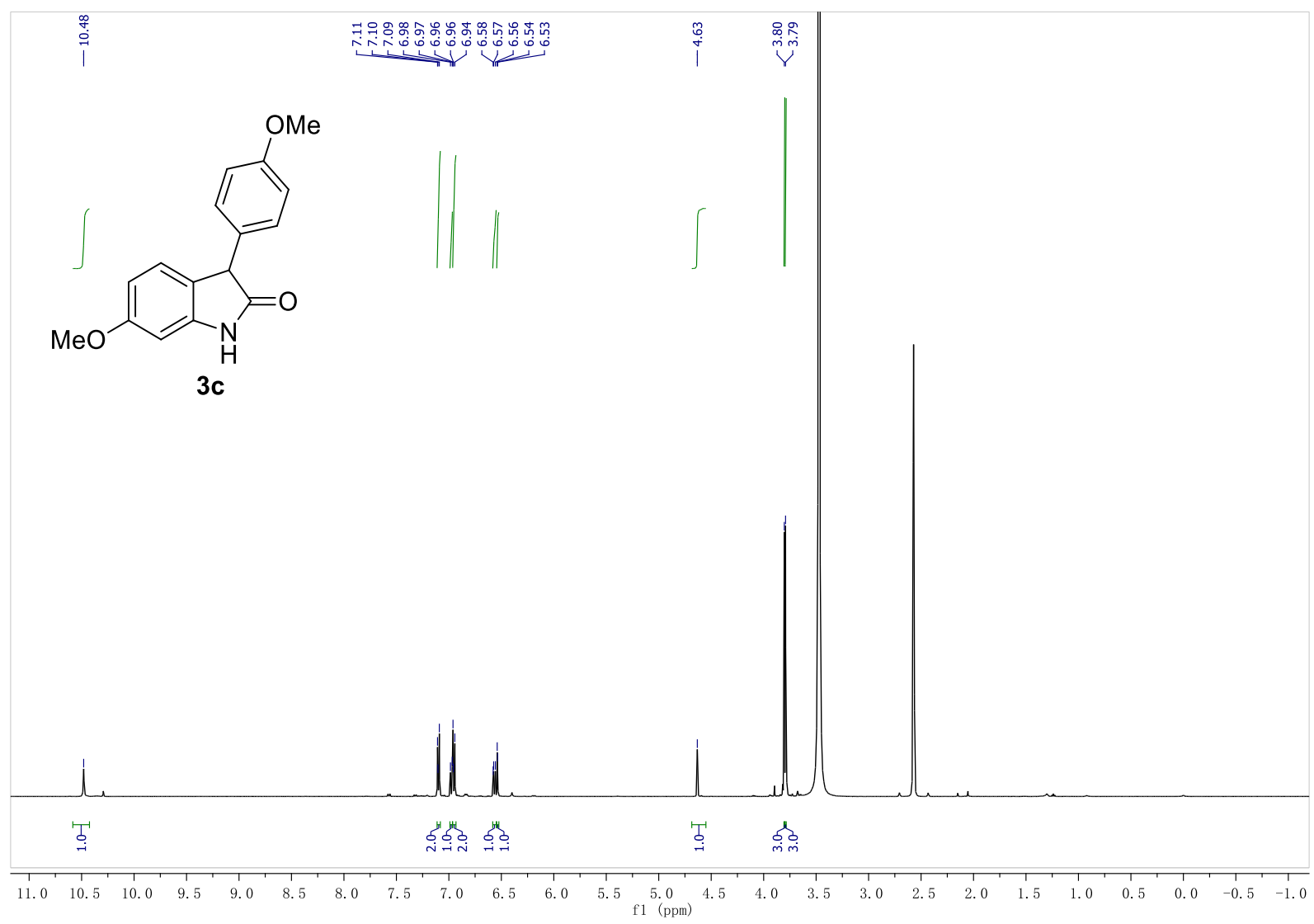

${ }^{1} \mathrm{H}$ NMR in DMSO- $d_{6}$ at $500 \mathrm{MHz}$

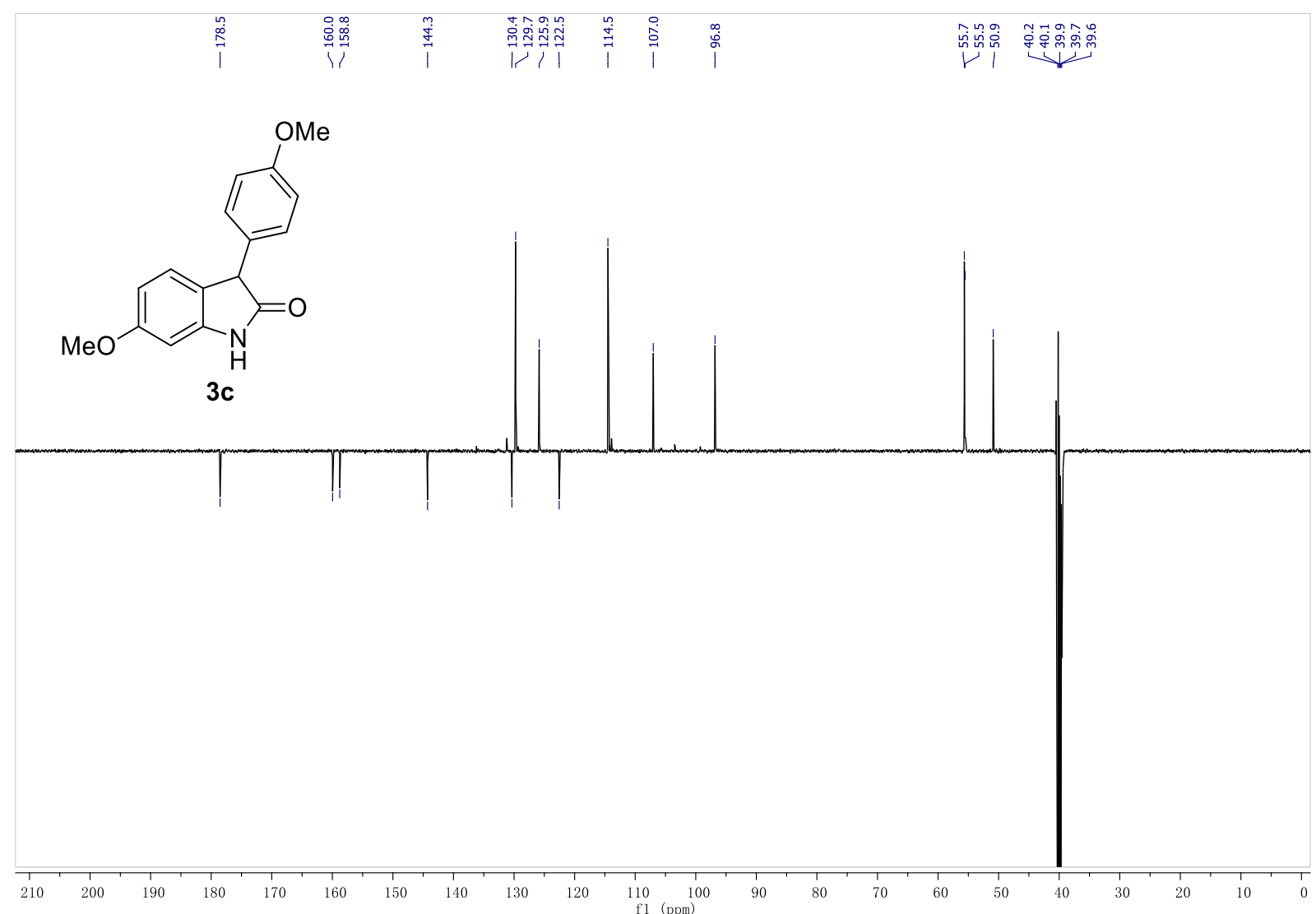

${ }^{13} \mathrm{C}\left\{{ }^{1} \mathrm{H}\right\} \mathrm{NMR}$ in DMSO- $d_{6}$ at $126 \mathrm{MHz}$ 


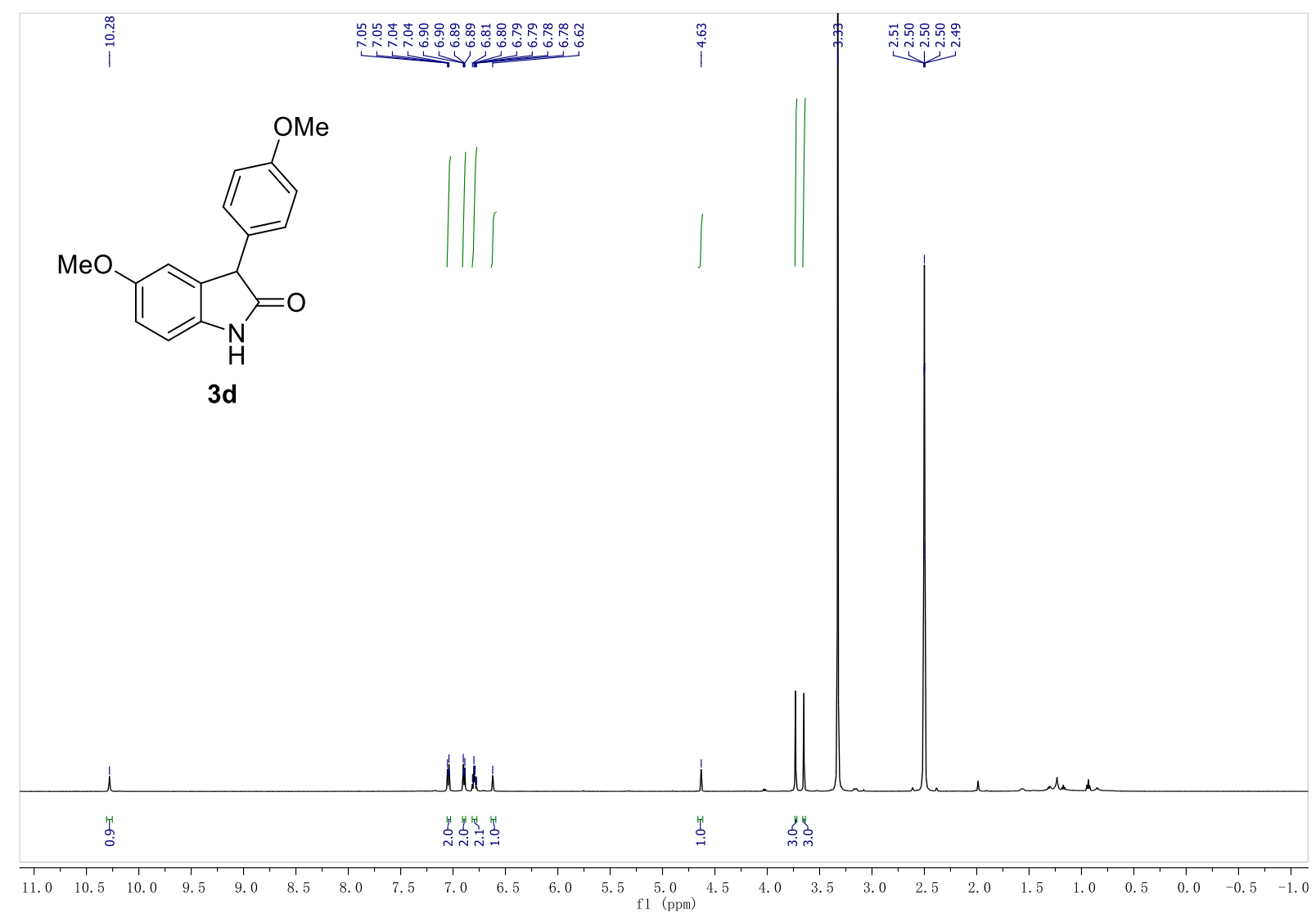

${ }^{1} \mathrm{H}$ NMR in DMSO- $d_{6}$ at $600 \mathrm{MHz}$

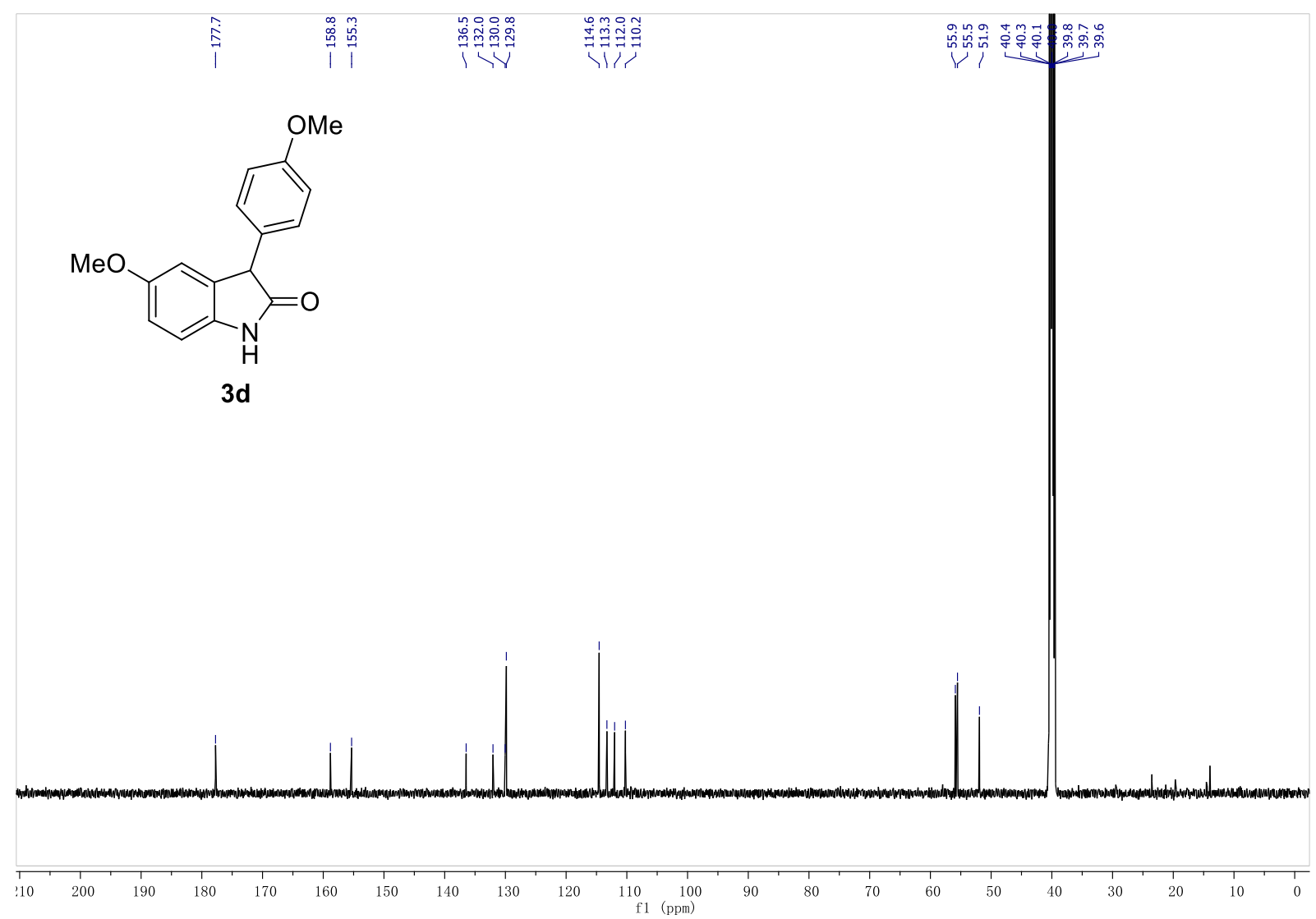

${ }^{13} \mathrm{C}\left\{{ }^{1} \mathrm{H}\right\} \mathrm{NMR}$ in DMSO- $d_{6}$ at $150 \mathrm{MHz}$ 


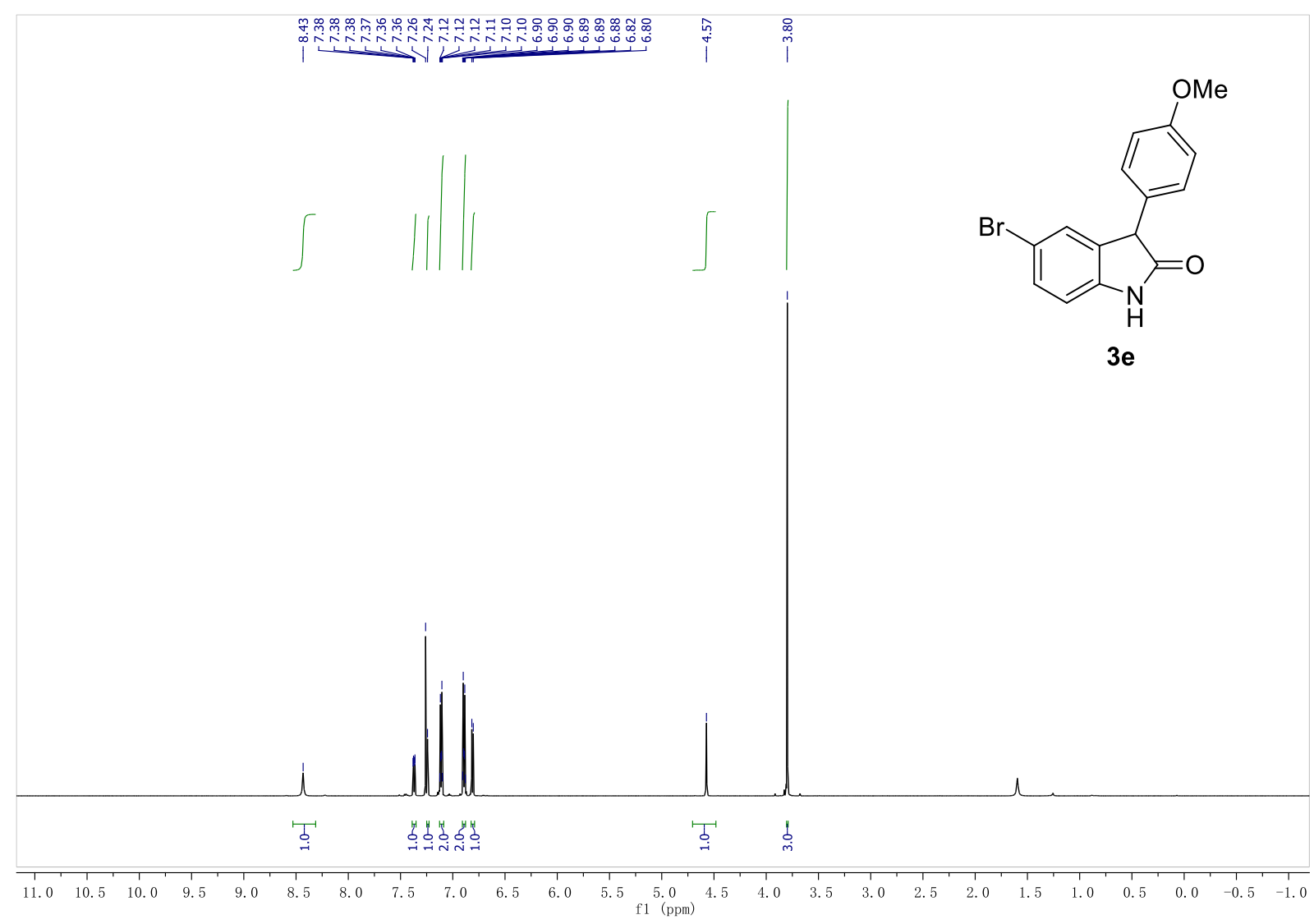

${ }^{1} \mathrm{H} \mathrm{NMR}$ in $\mathrm{CDCl}_{3}$ at $600 \mathrm{MHz}$

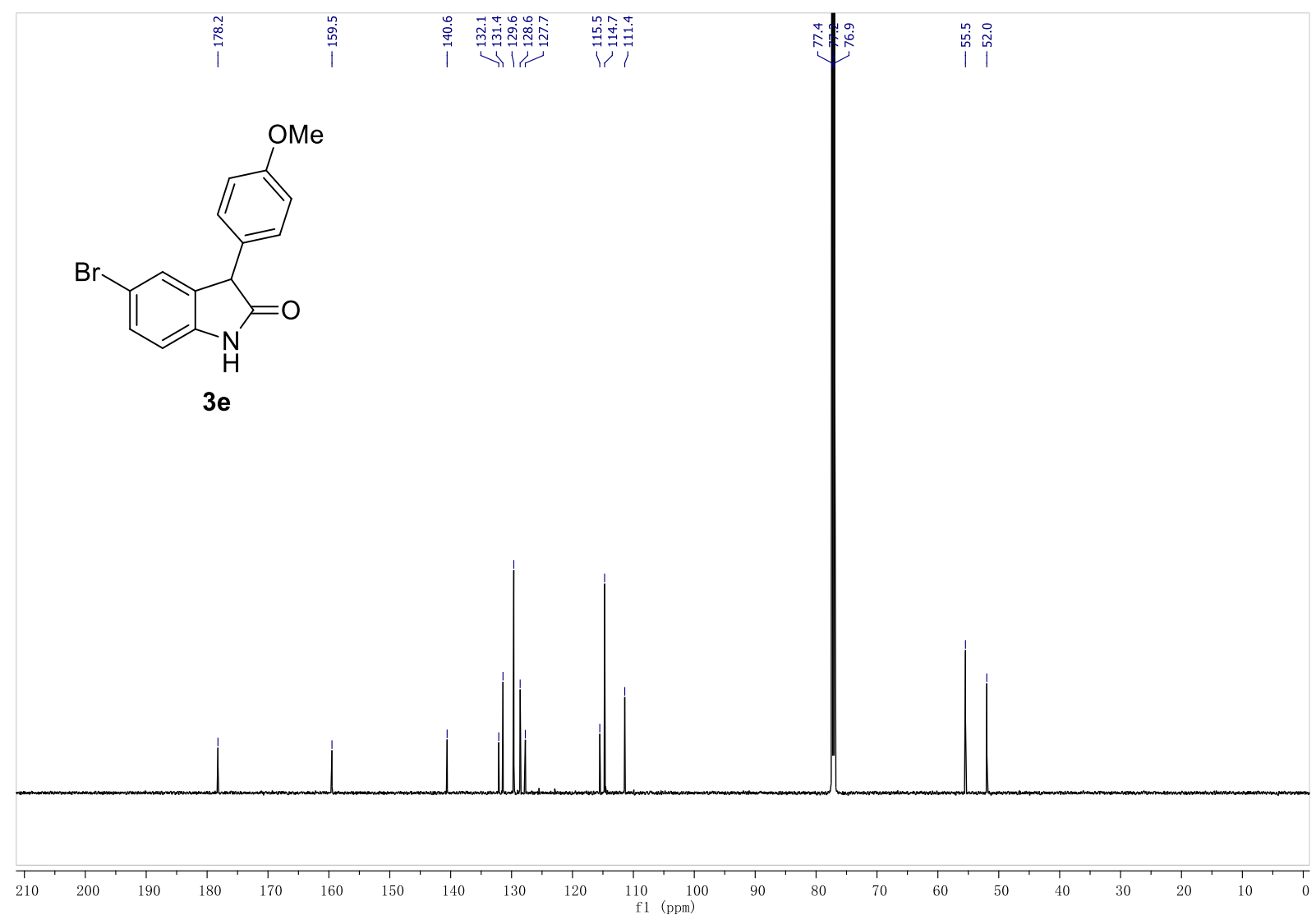

${ }^{13} \mathrm{C}\left\{{ }^{1} \mathrm{H}\right\} \mathrm{NMR}$ in $\mathrm{CDCl}_{3}$ at $150 \mathrm{MHz}$ 


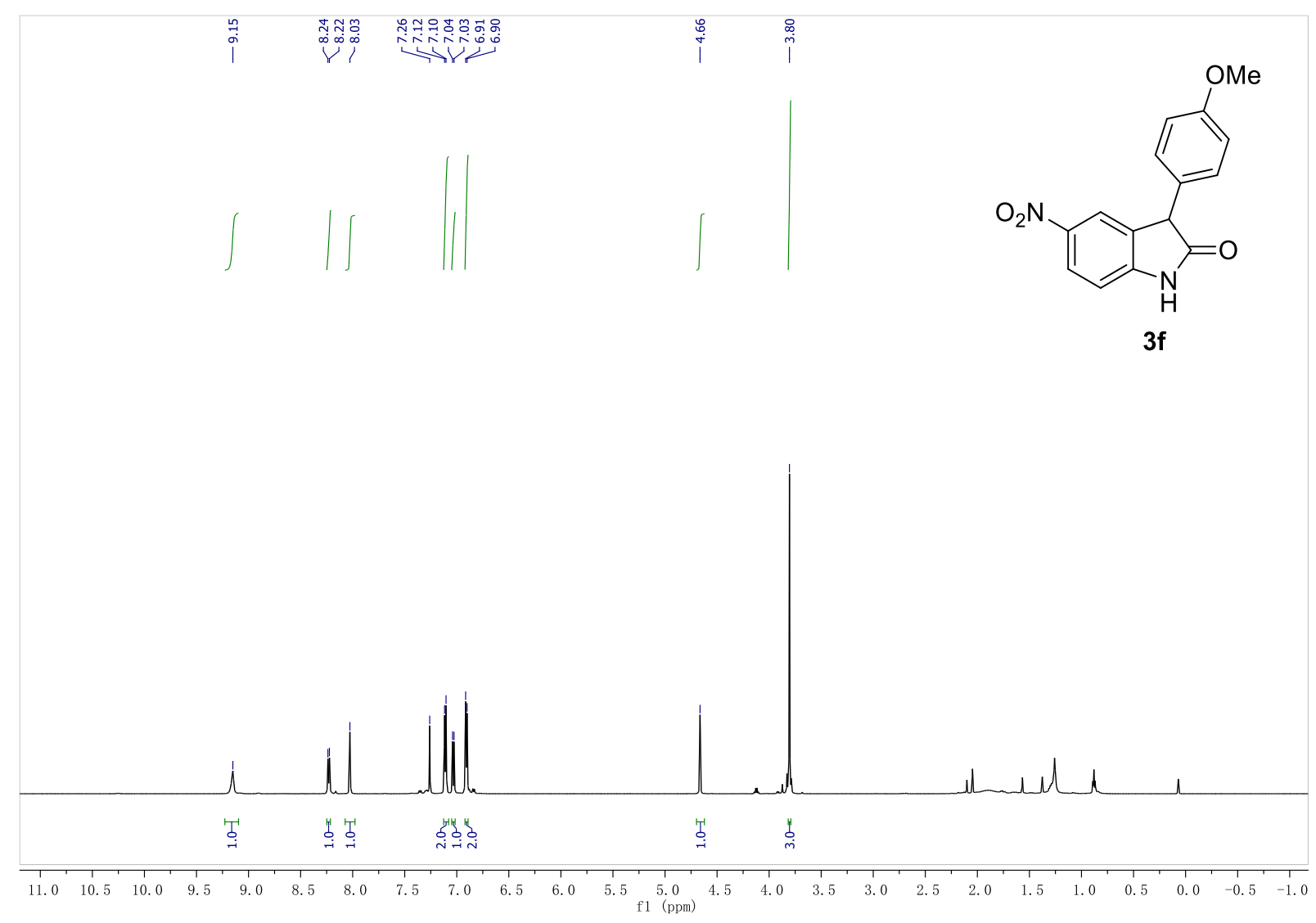

${ }^{1} \mathrm{H} \mathrm{NMR}$ in $\mathrm{CDCl}_{3}$ at $600 \mathrm{MHz}$

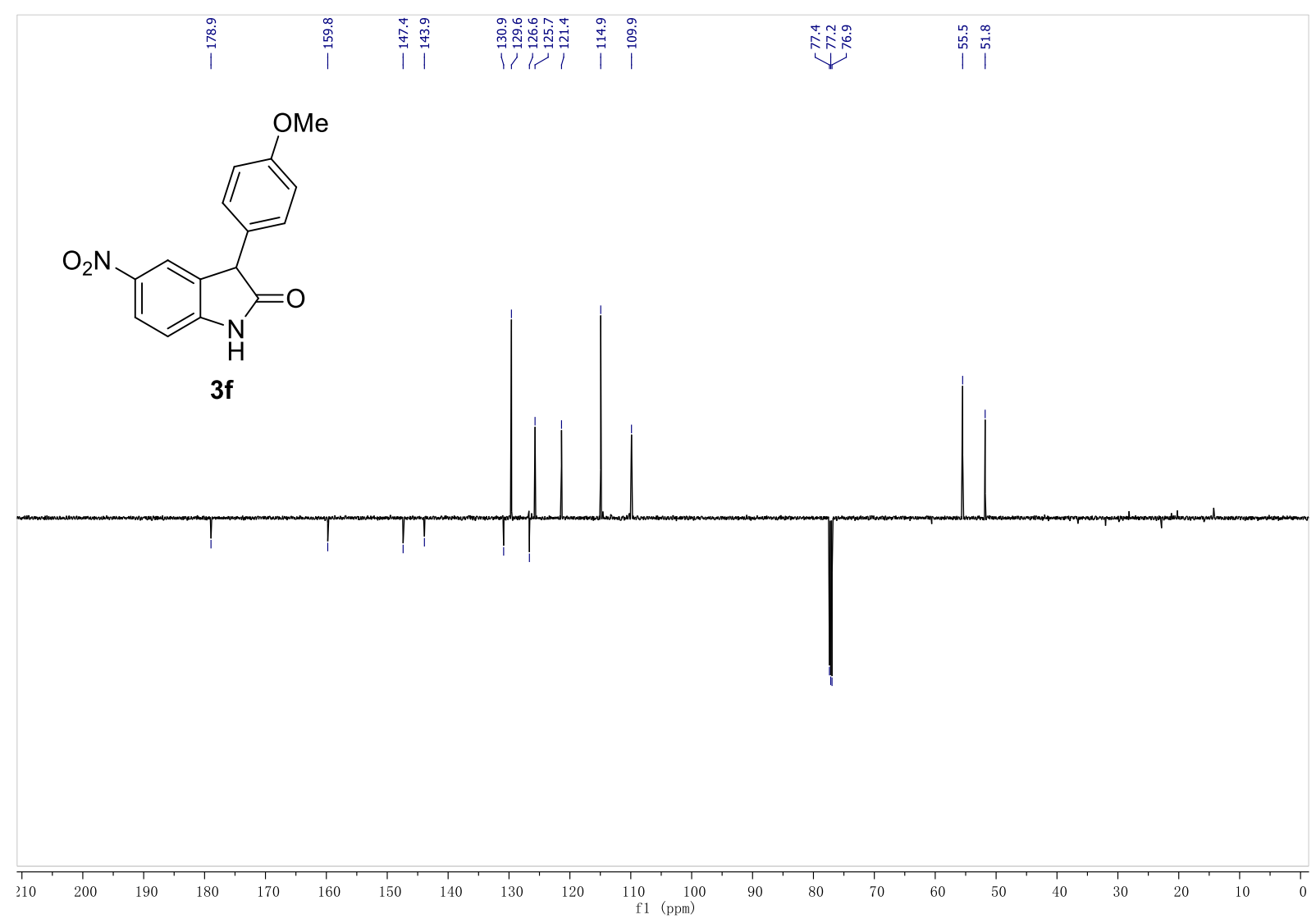

${ }^{13} \mathrm{C}\left\{{ }^{1} \mathrm{H}\right\} \mathrm{NMR}$ in $\mathrm{CDCl}_{3}$ at $150 \mathrm{MHz}$ 


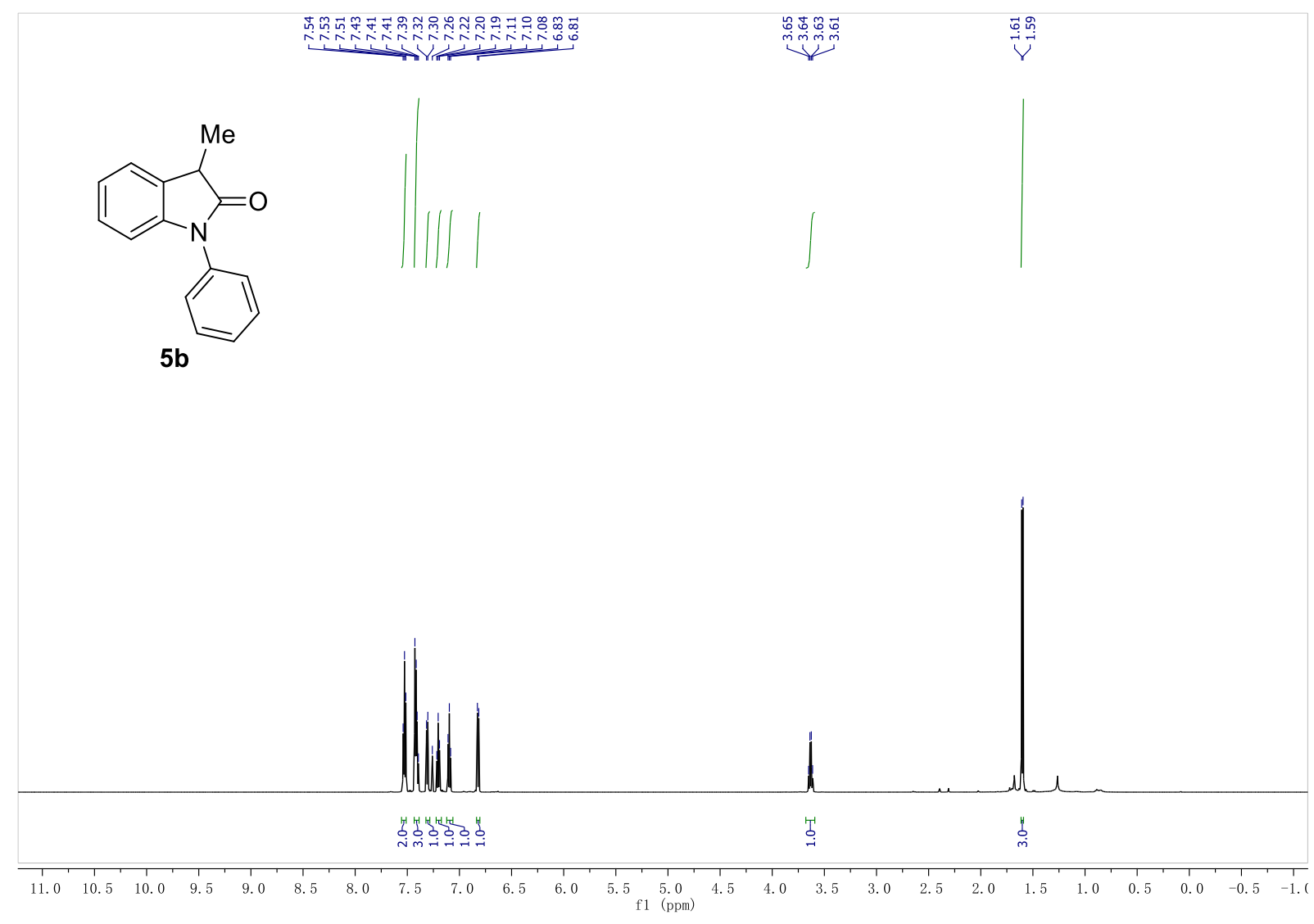

${ }^{1} \mathrm{H} \mathrm{NMR}$ in $\mathrm{CDCl}_{3}$ at $600 \mathrm{MHz}$

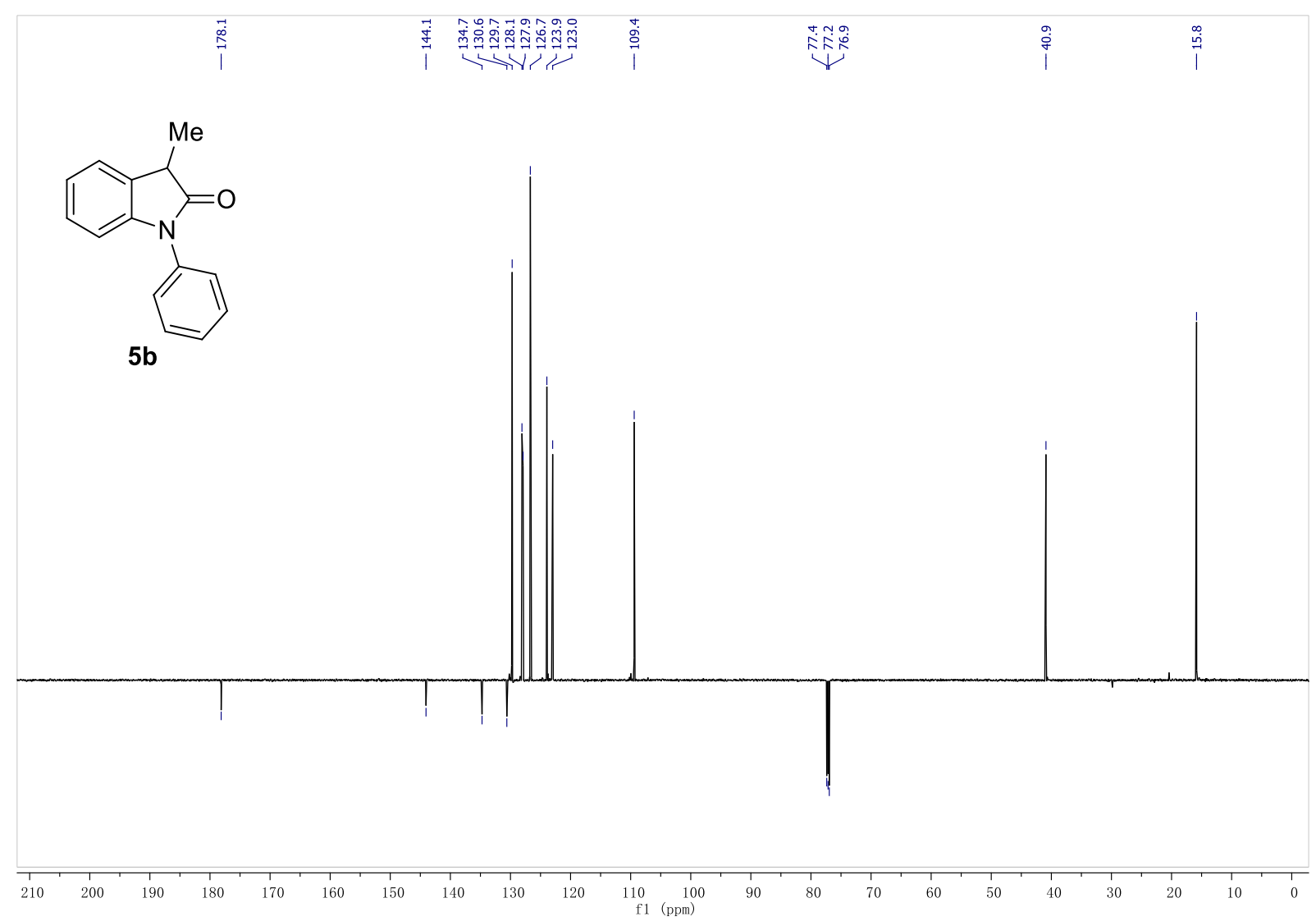

${ }^{13} \mathrm{C}\left\{{ }^{1} \mathrm{H}\right\}$ NMR in $\mathrm{CDCl}_{3}$ at $150 \mathrm{MHz}$ 


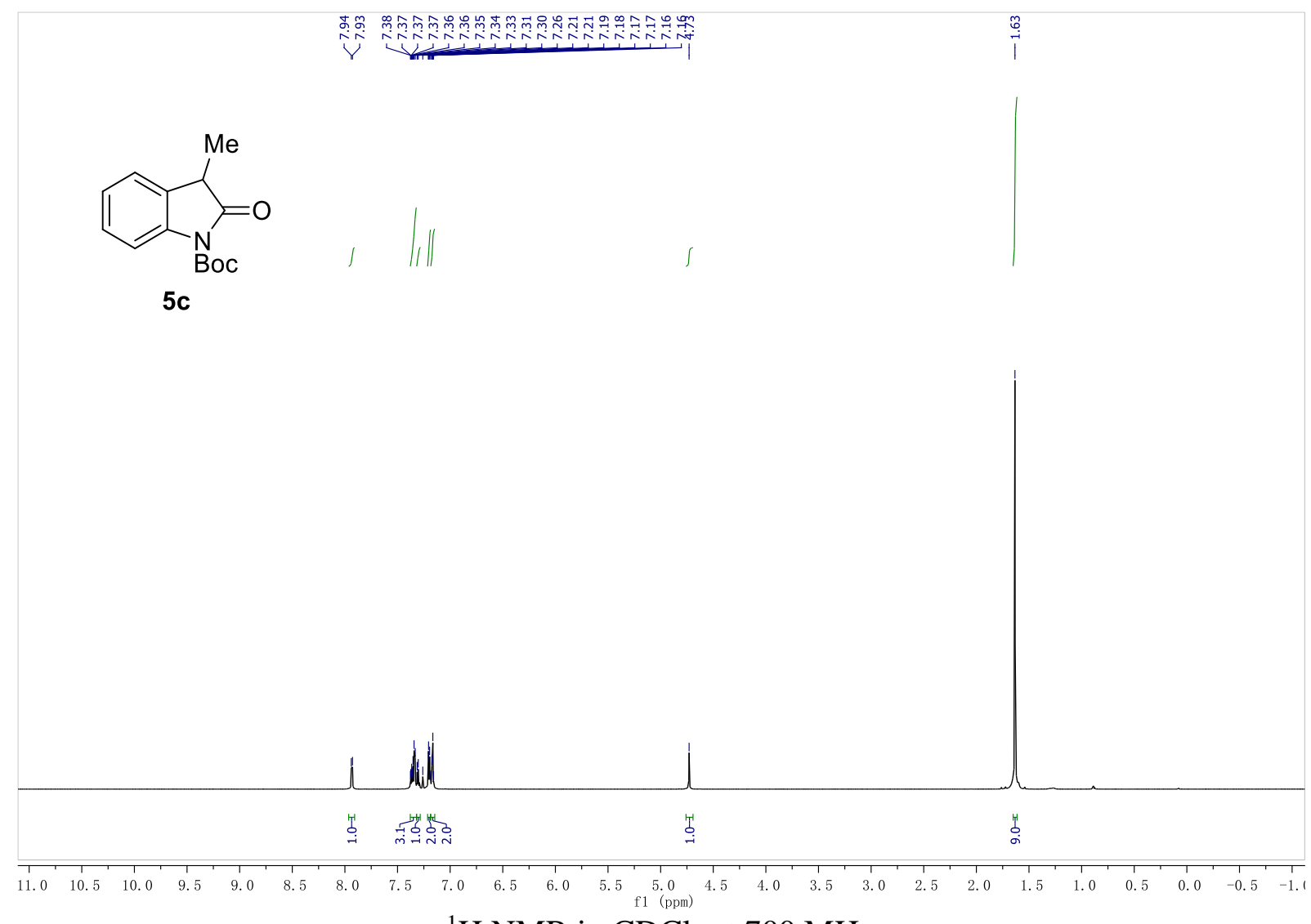

${ }^{1} \mathrm{H} \mathrm{NMR} \mathrm{in} \mathrm{CDCl}_{3}$ at $700 \mathrm{MHz}$

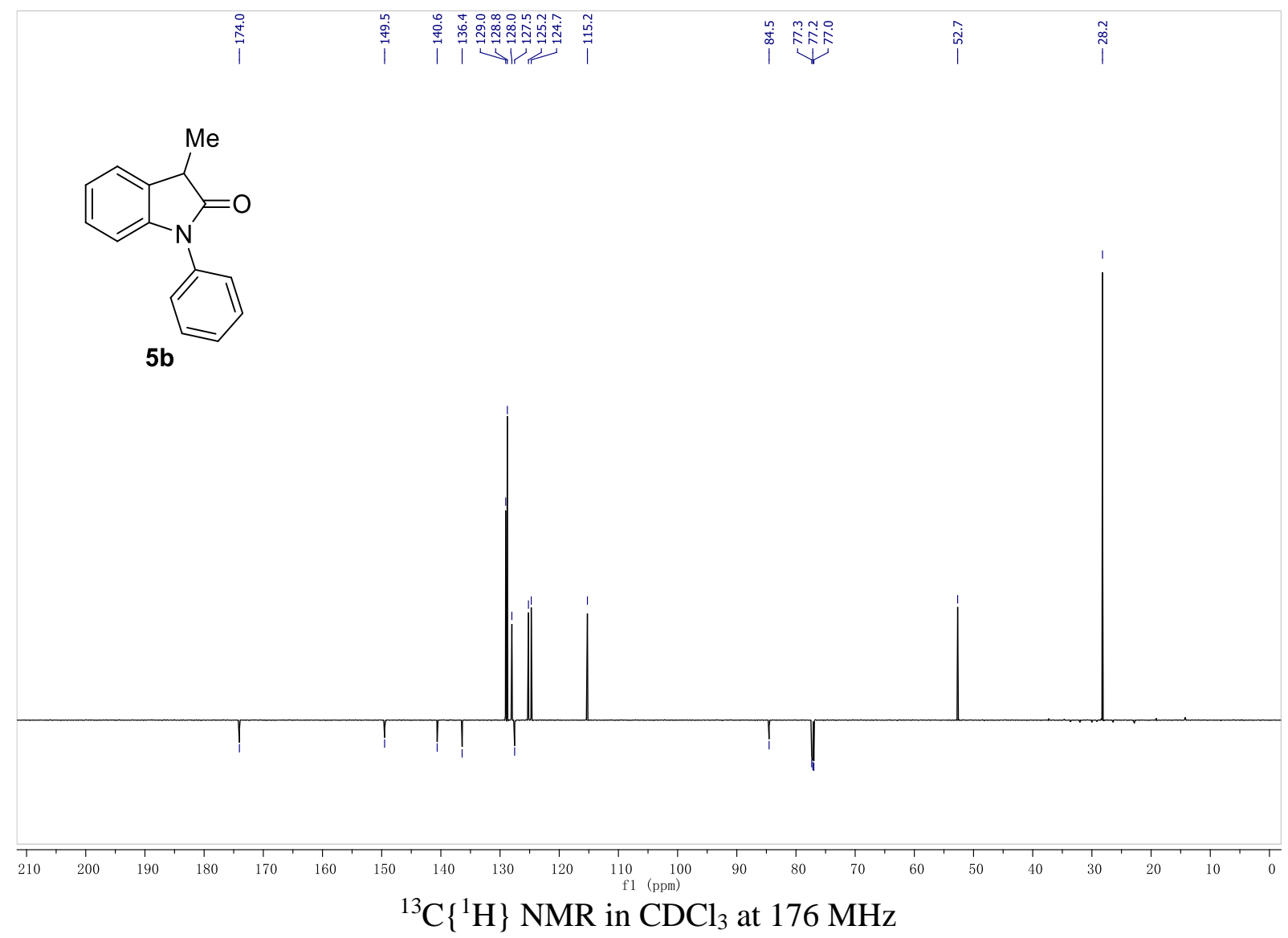




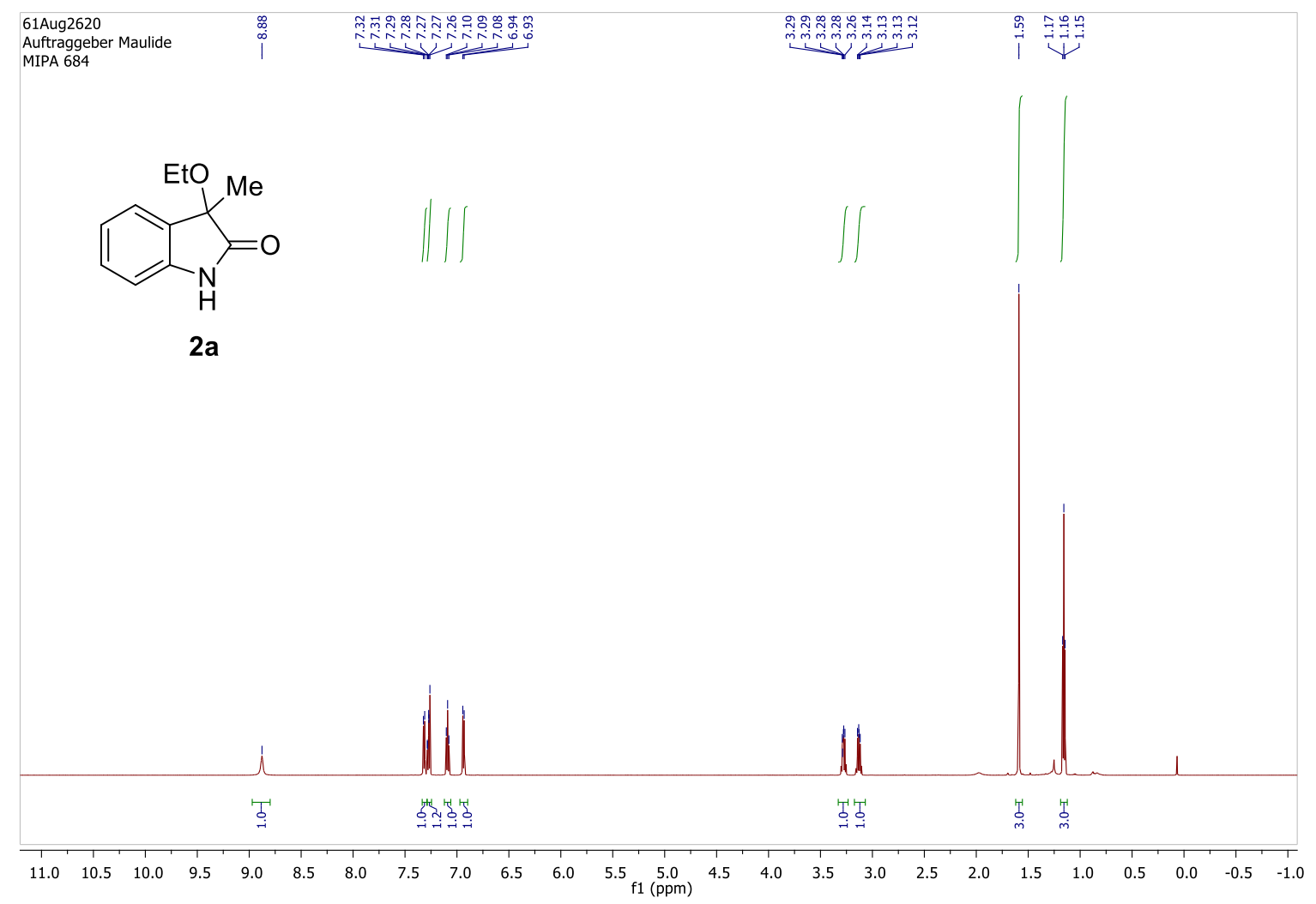

${ }^{1} \mathrm{H}$ NMR in $\mathrm{CDCl}_{3}$ at $600 \mathrm{MHz}$

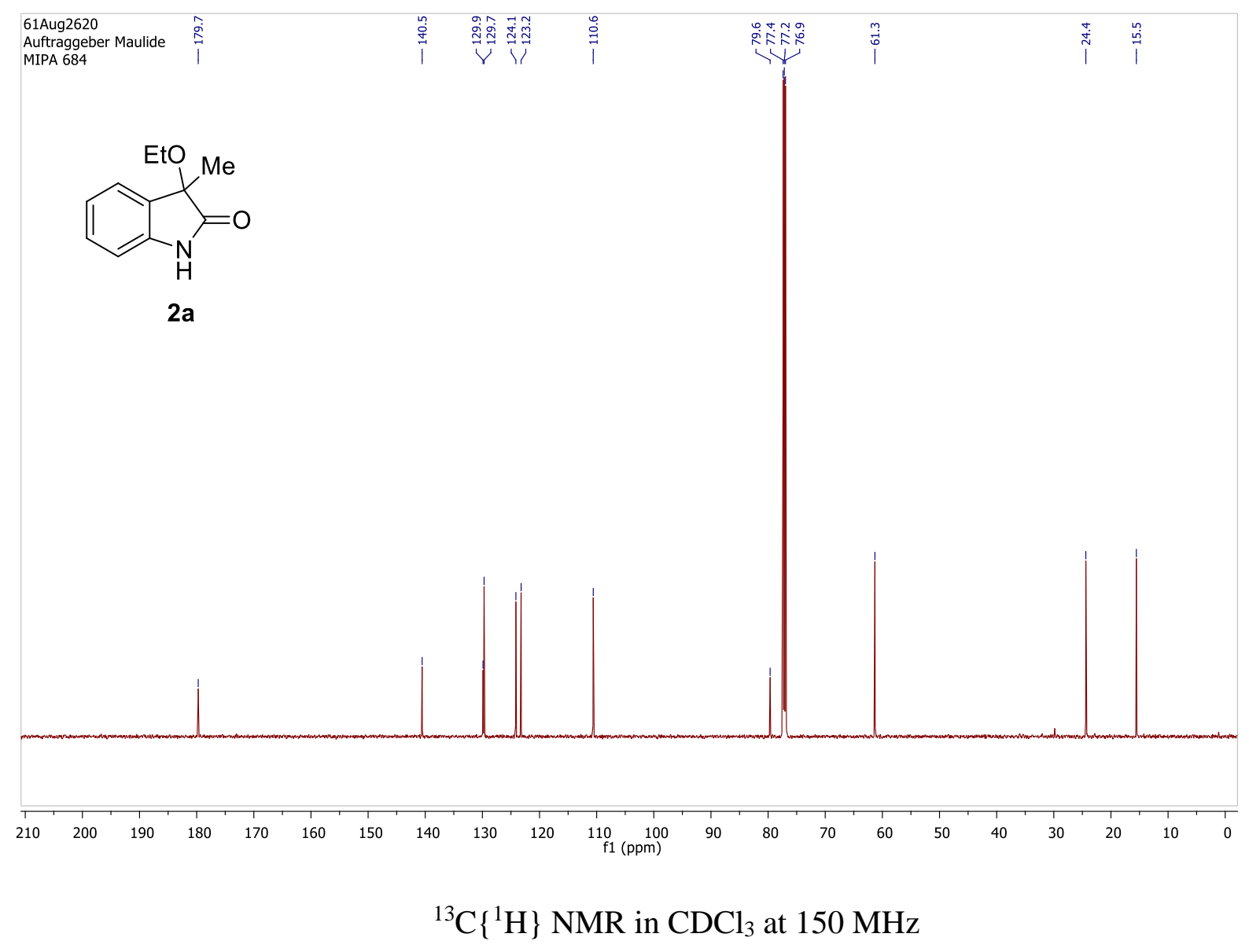




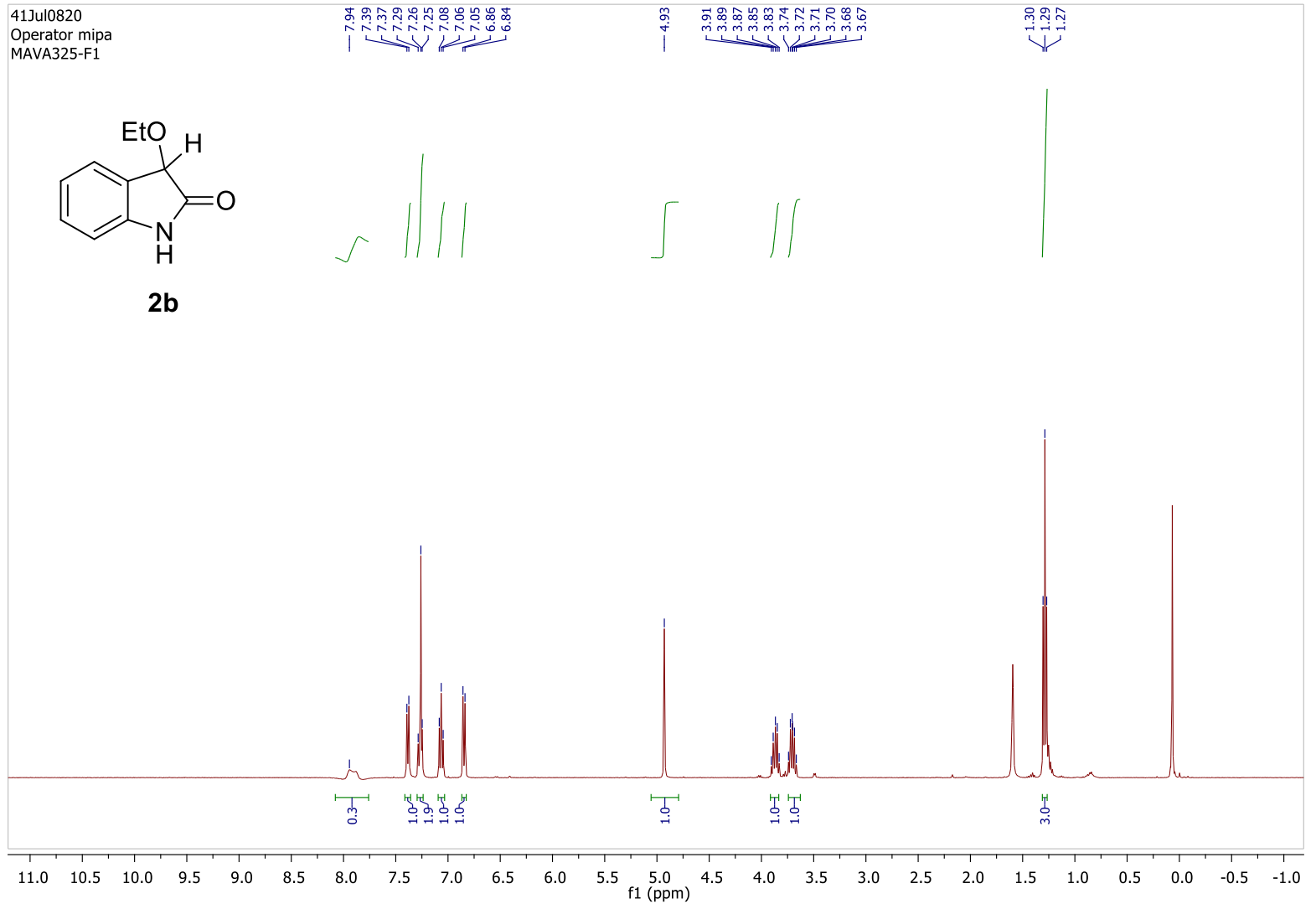

${ }^{1} \mathrm{H} \mathrm{NMR} \mathrm{in} \mathrm{CDCl}_{3}$ at $400 \mathrm{MHz}$

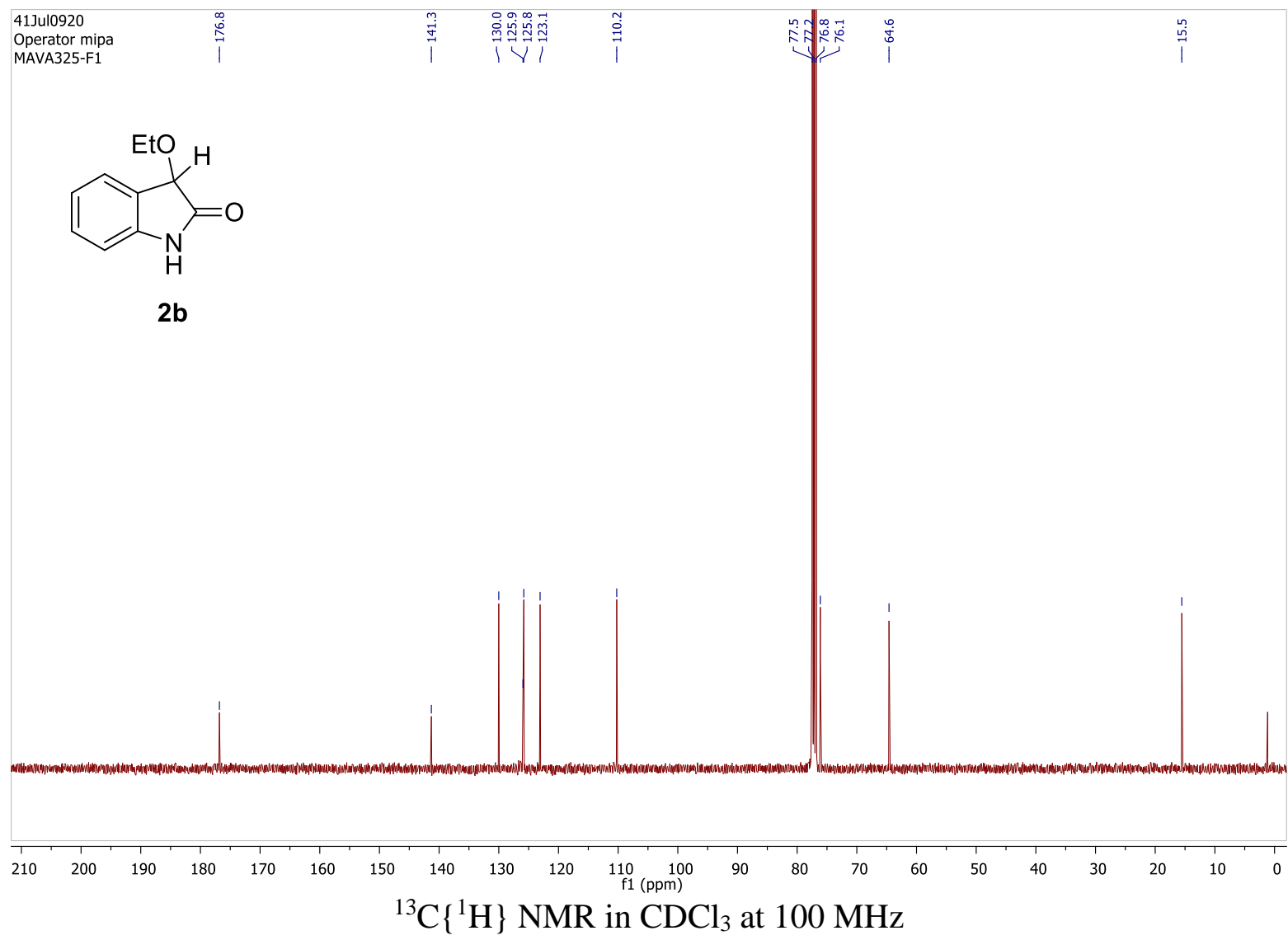




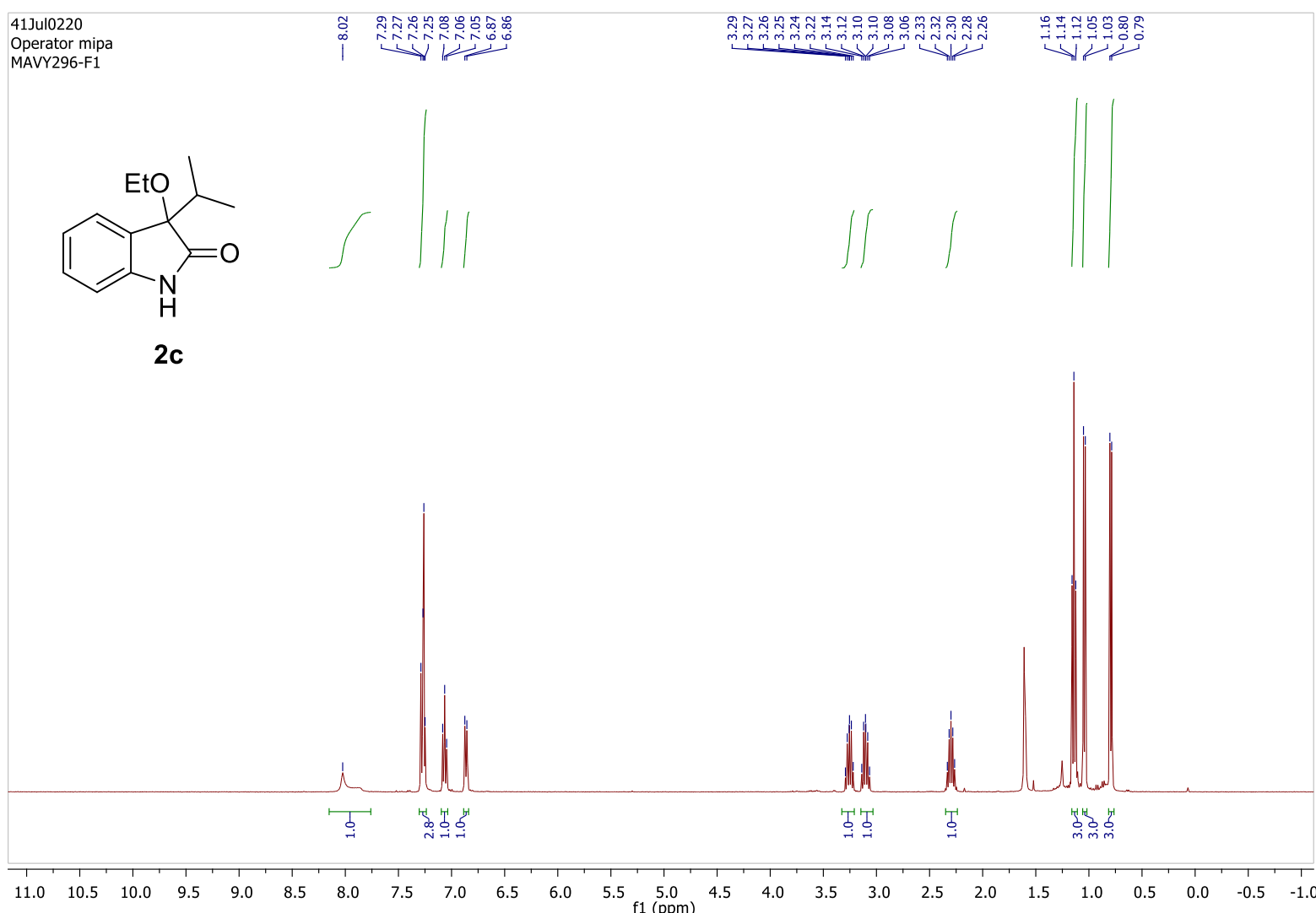

${ }^{1} \mathrm{H} \mathrm{NMR} \mathrm{in} \mathrm{CDCl}_{3}$ at $400 \mathrm{MHz}$

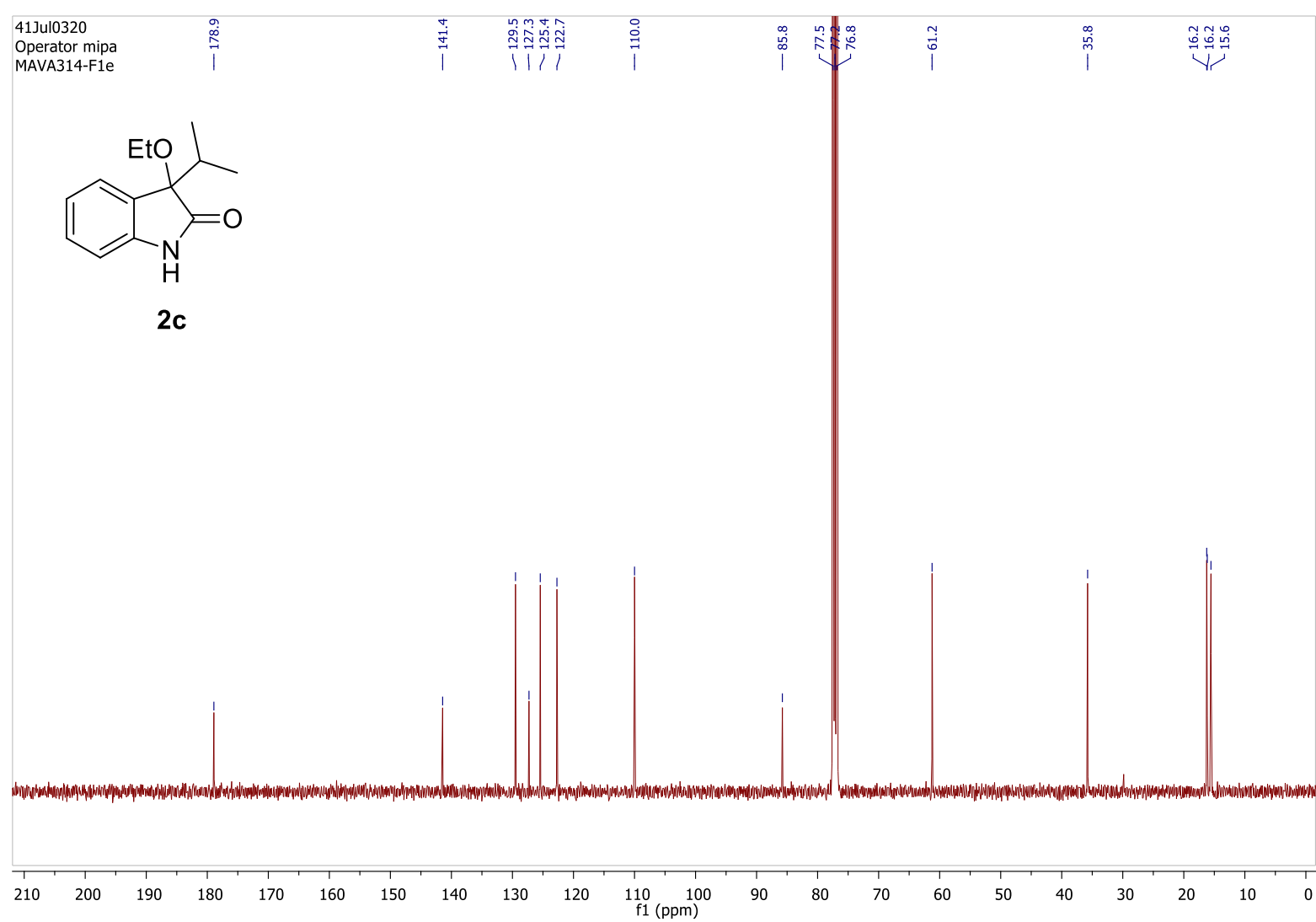

${ }^{13} \mathrm{C}\left\{{ }^{1} \mathrm{H}\right\} \mathrm{NMR}$ in $\mathrm{CDCl}_{3}$ at $100 \mathrm{MHz}$ 


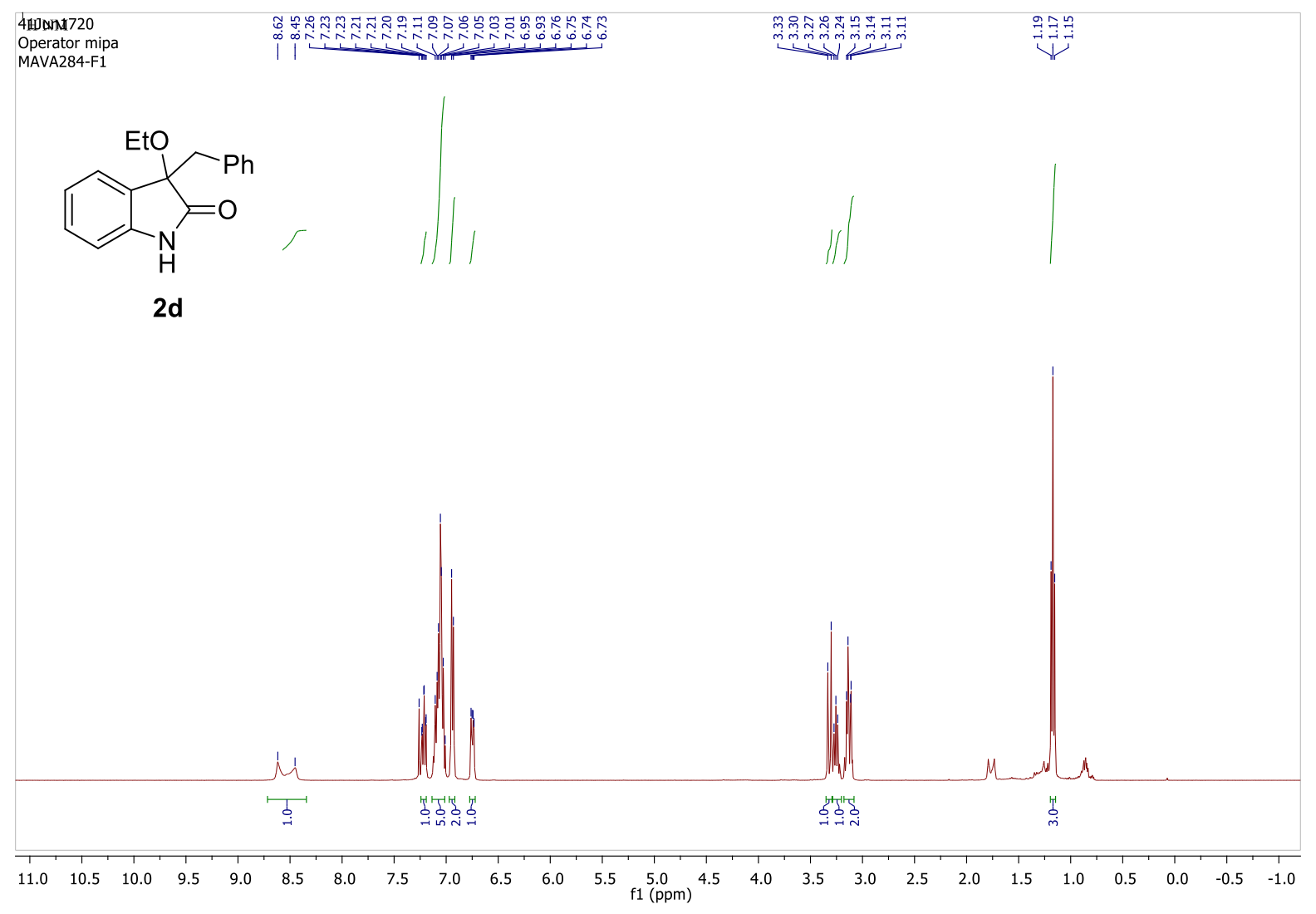

${ }^{1} \mathrm{H} \mathrm{NMR}$ in $\mathrm{CDCl}_{3}$ at $400 \mathrm{MHz}$

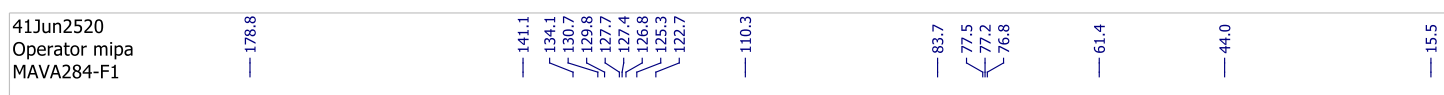

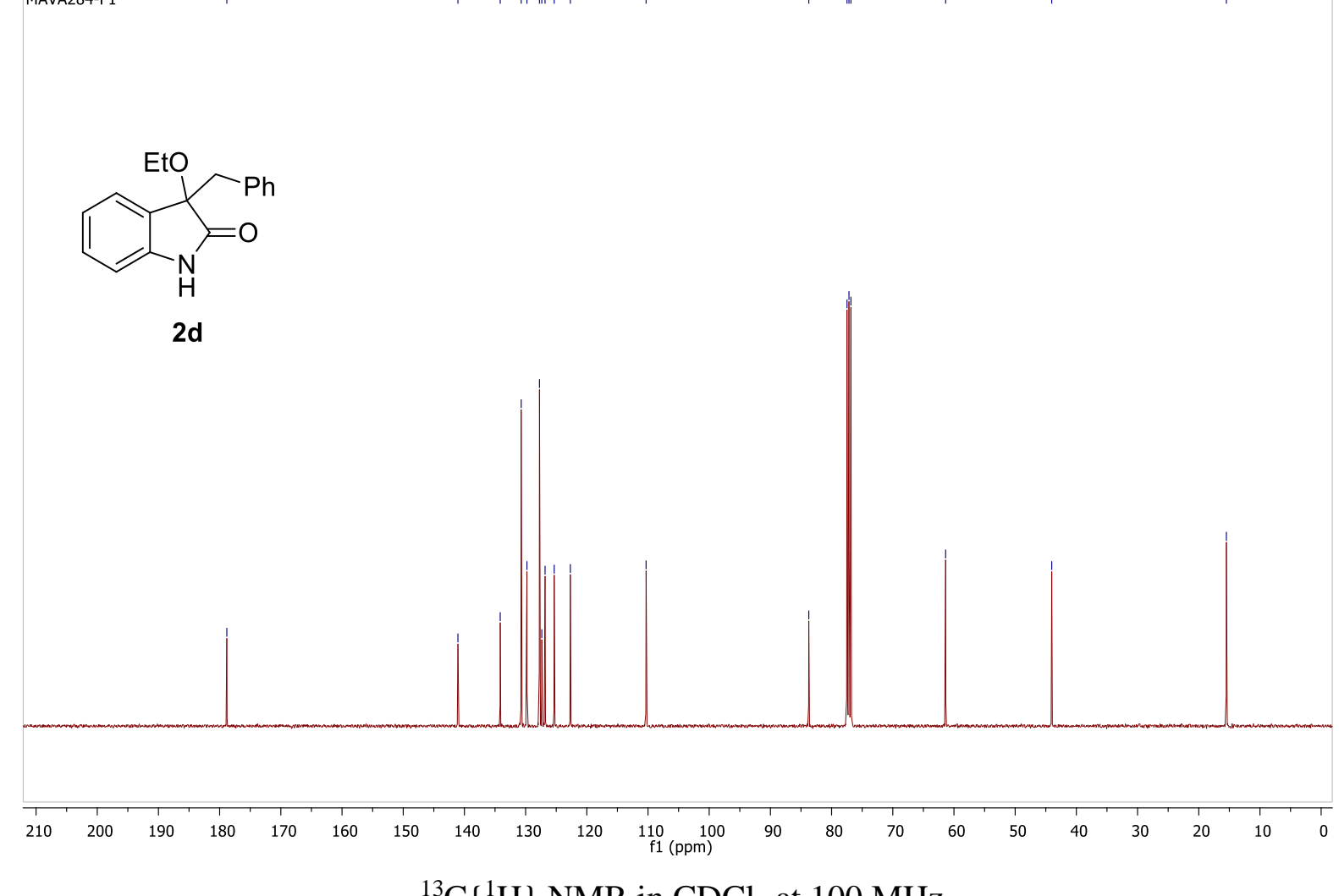

${ }^{13} \mathrm{C}\left\{{ }^{1} \mathrm{H}\right\}$ NMR in $\mathrm{CDCl}_{3}$ at $100 \mathrm{MHz}$ 


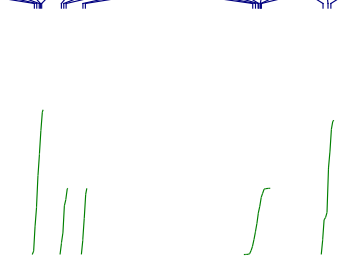<smiles>C=CCC1(OCC)C(=O)Nc2ccccc21</smiles>

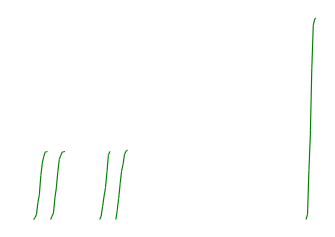

2e

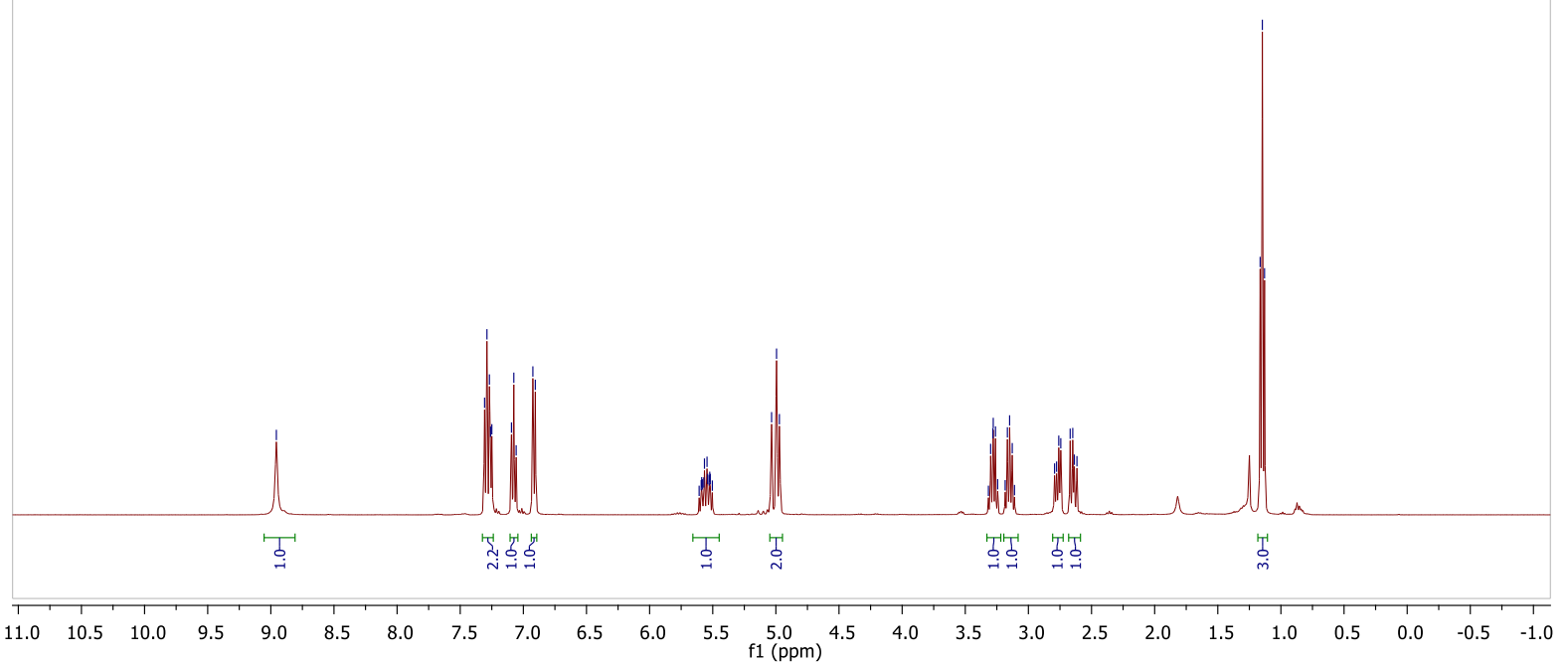

${ }^{1} \mathrm{H} \mathrm{NMR} \mathrm{in} \mathrm{CDCl}_{3}$ at $400 \mathrm{MHz}$

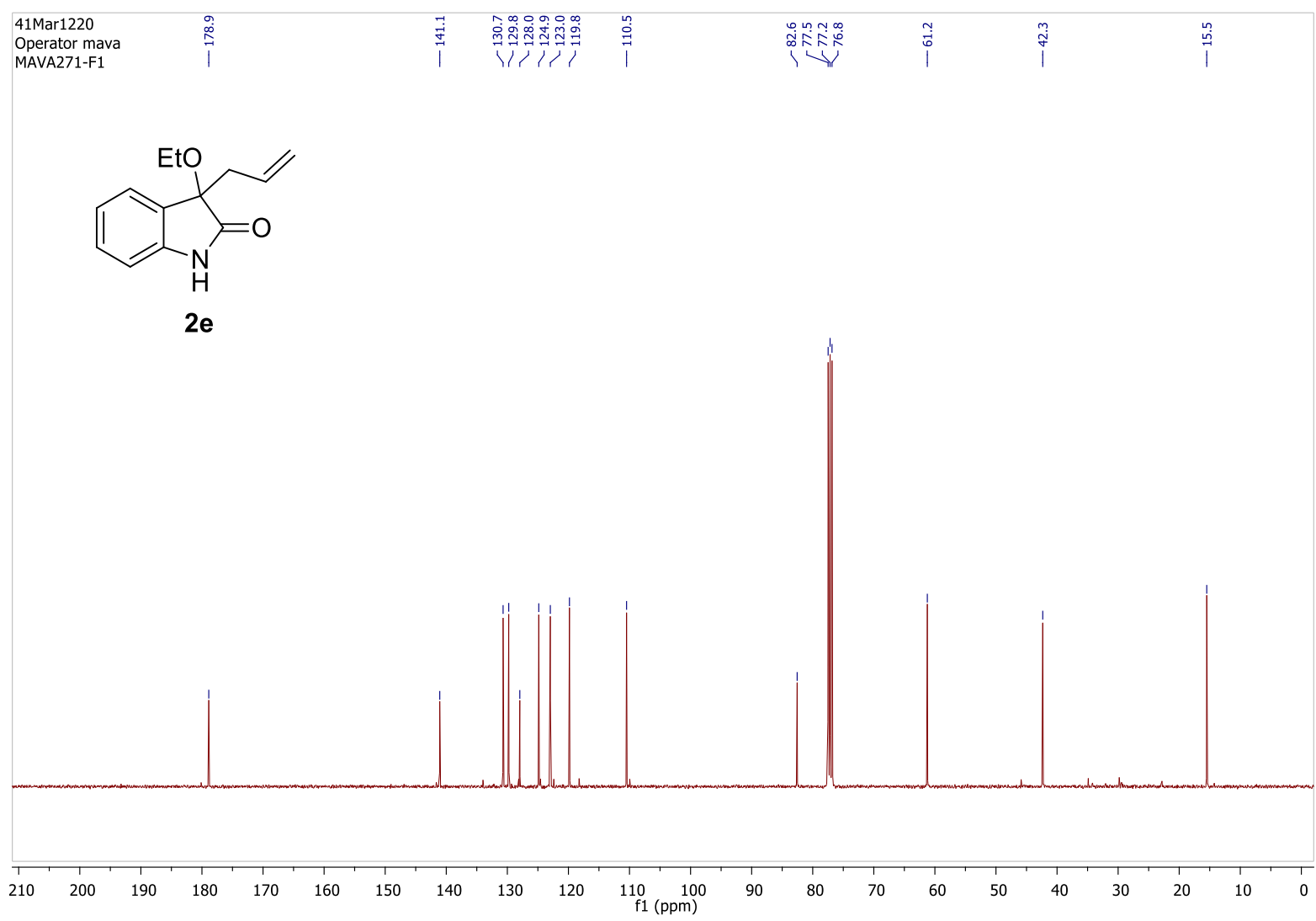

${ }^{13} \mathrm{C}\left\{{ }^{1} \mathrm{H}\right\} \mathrm{NMR}$ in $\mathrm{CDCl}_{3}$ at $100 \mathrm{MHz}$ 
<smiles>CCOC1(C2CCCC2)C(=O)Nc2ccccc21</smiles>

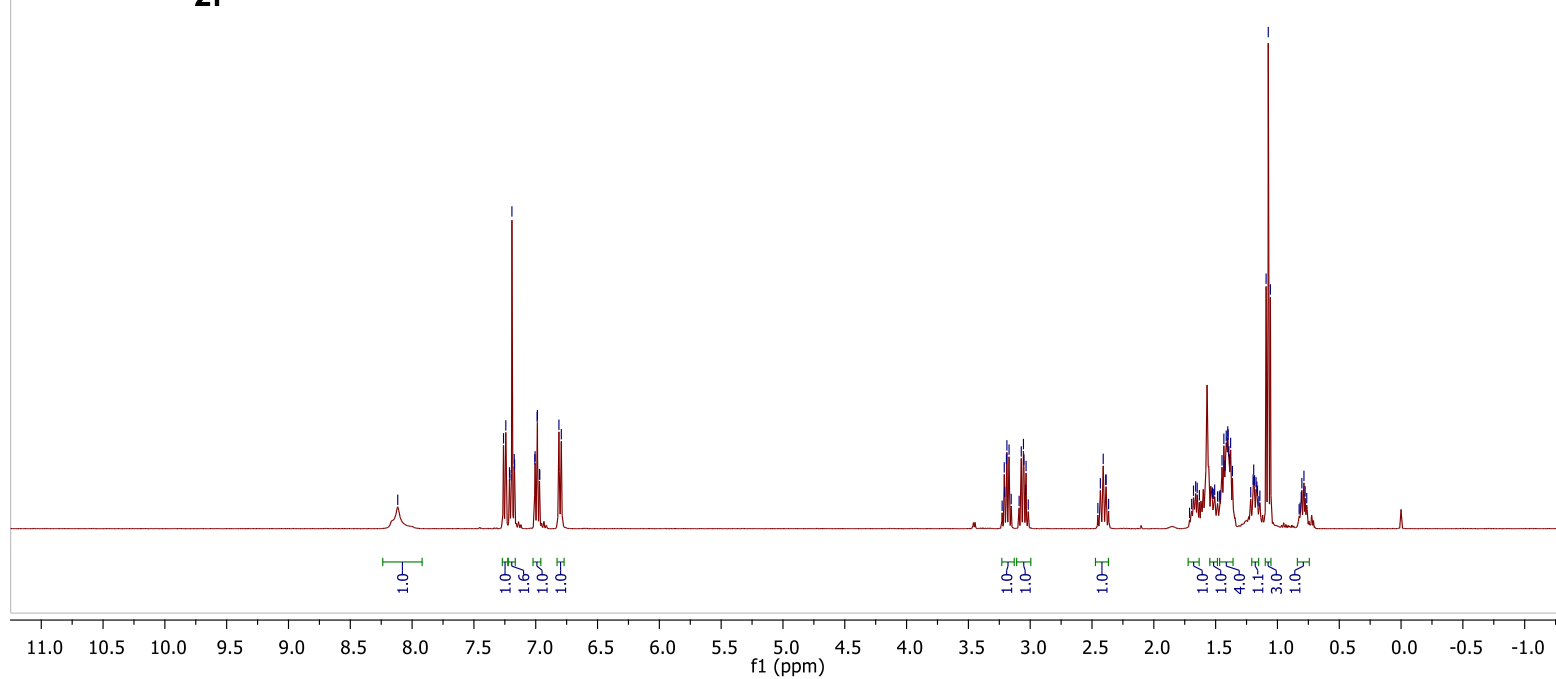

${ }^{1} \mathrm{H} \mathrm{NMR}$ in $\mathrm{CDCl}_{3}$ at $400 \mathrm{MHz}$

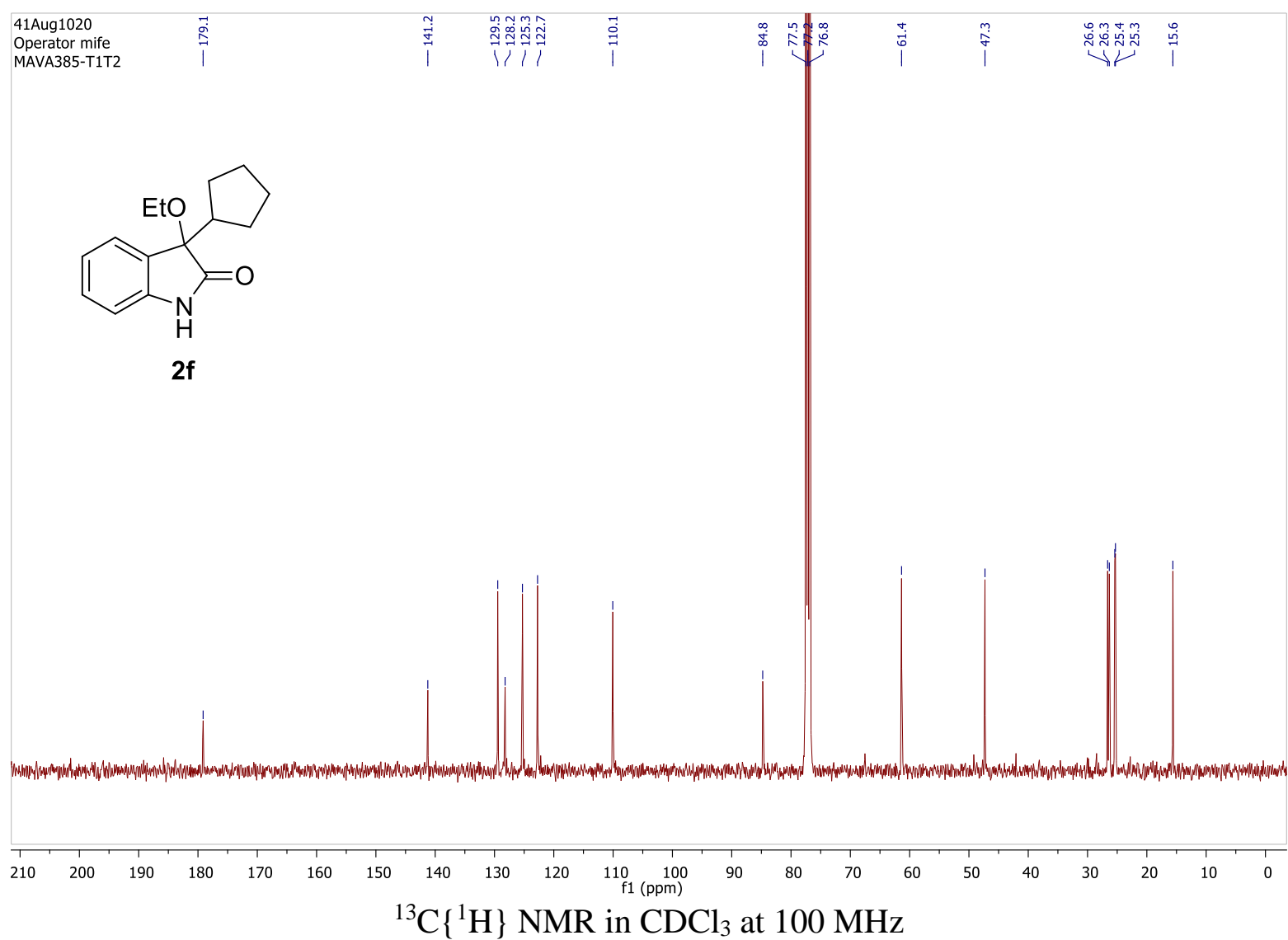




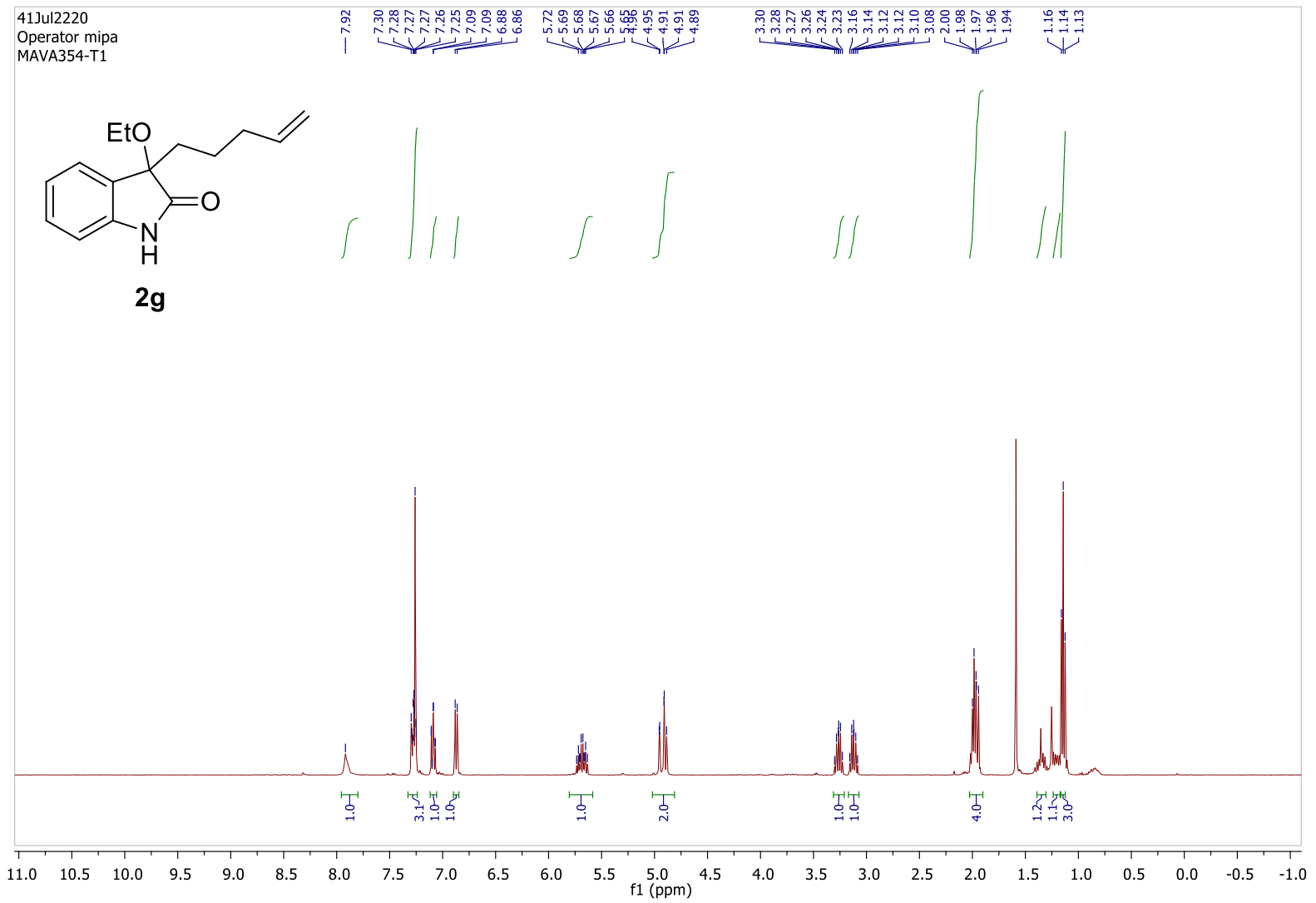

${ }^{1} \mathrm{H} \mathrm{NMR} \mathrm{in} \mathrm{CDCl}_{3}$ at $400 \mathrm{MHz}$

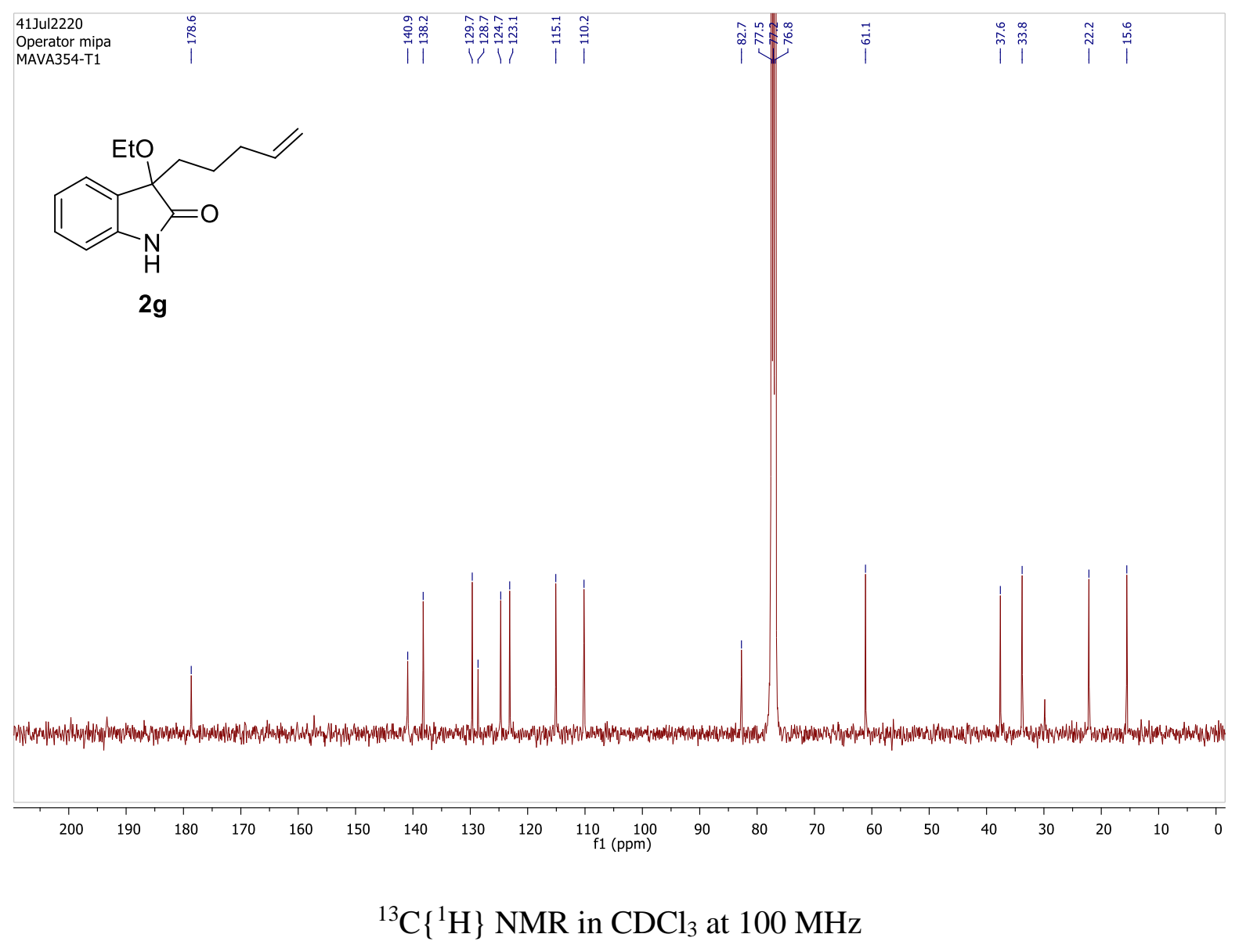




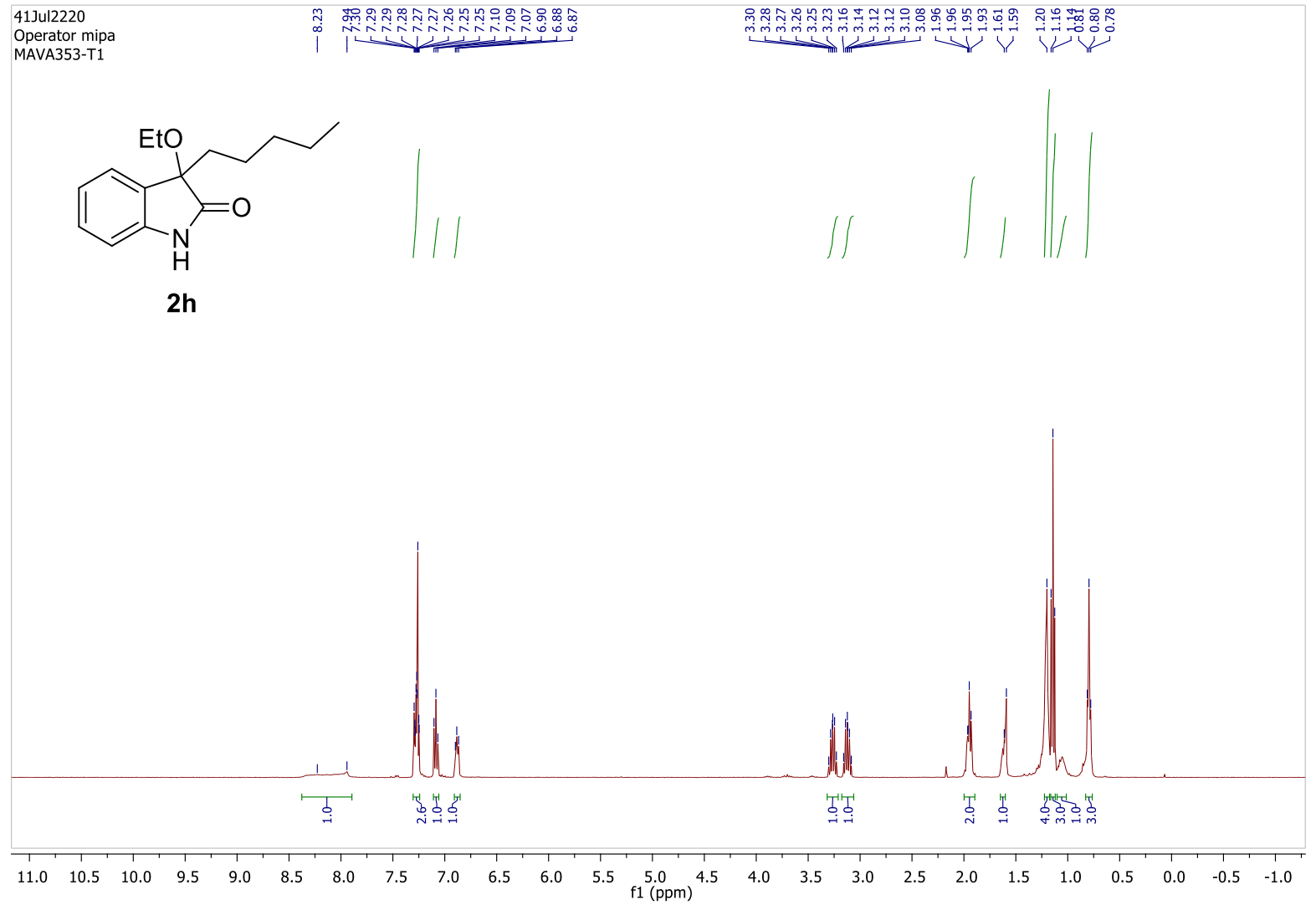

${ }^{1} \mathrm{H} \mathrm{NMR}$ in $\mathrm{CDCl}_{3}$ at $400 \mathrm{MHz}$

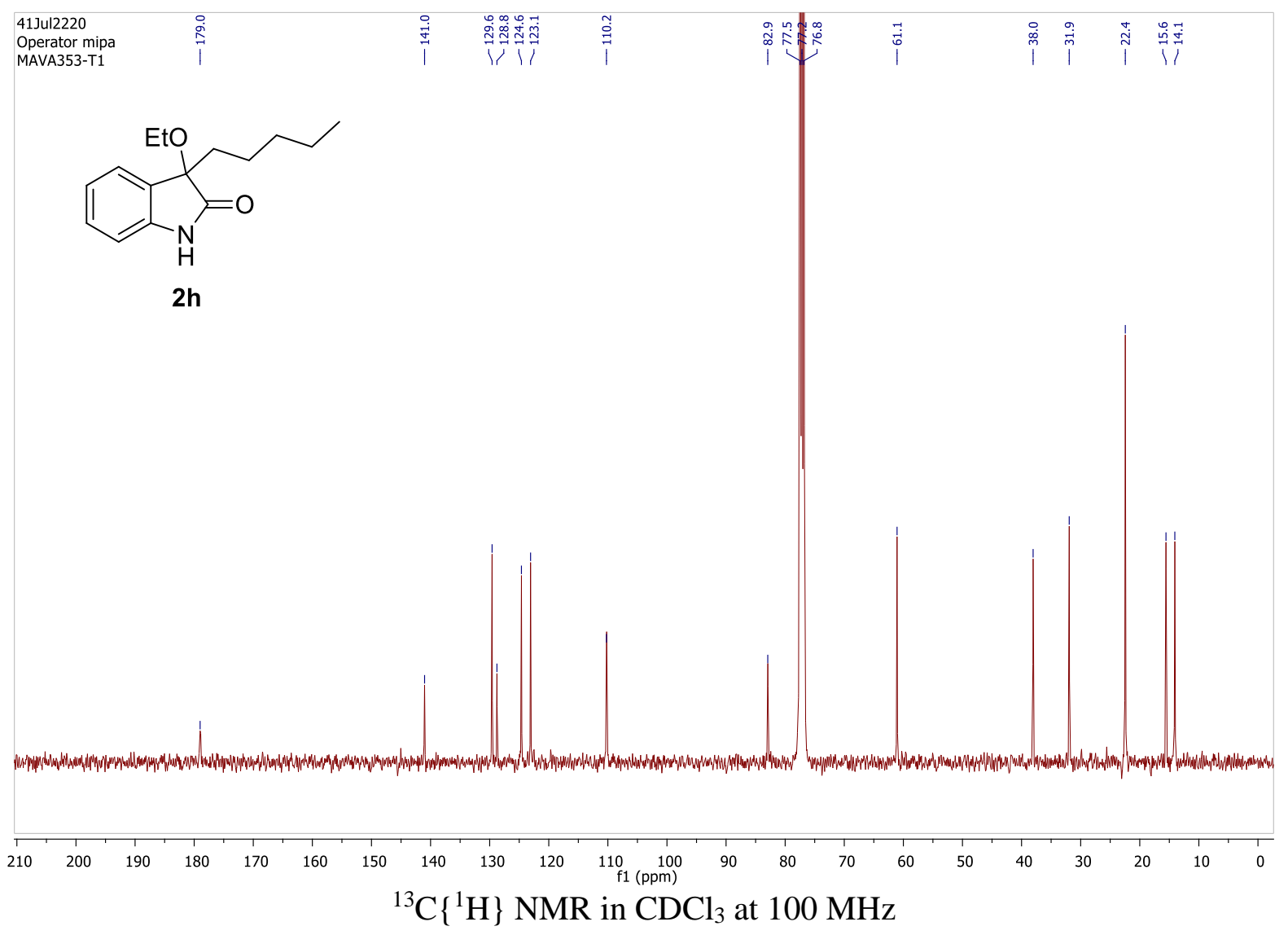




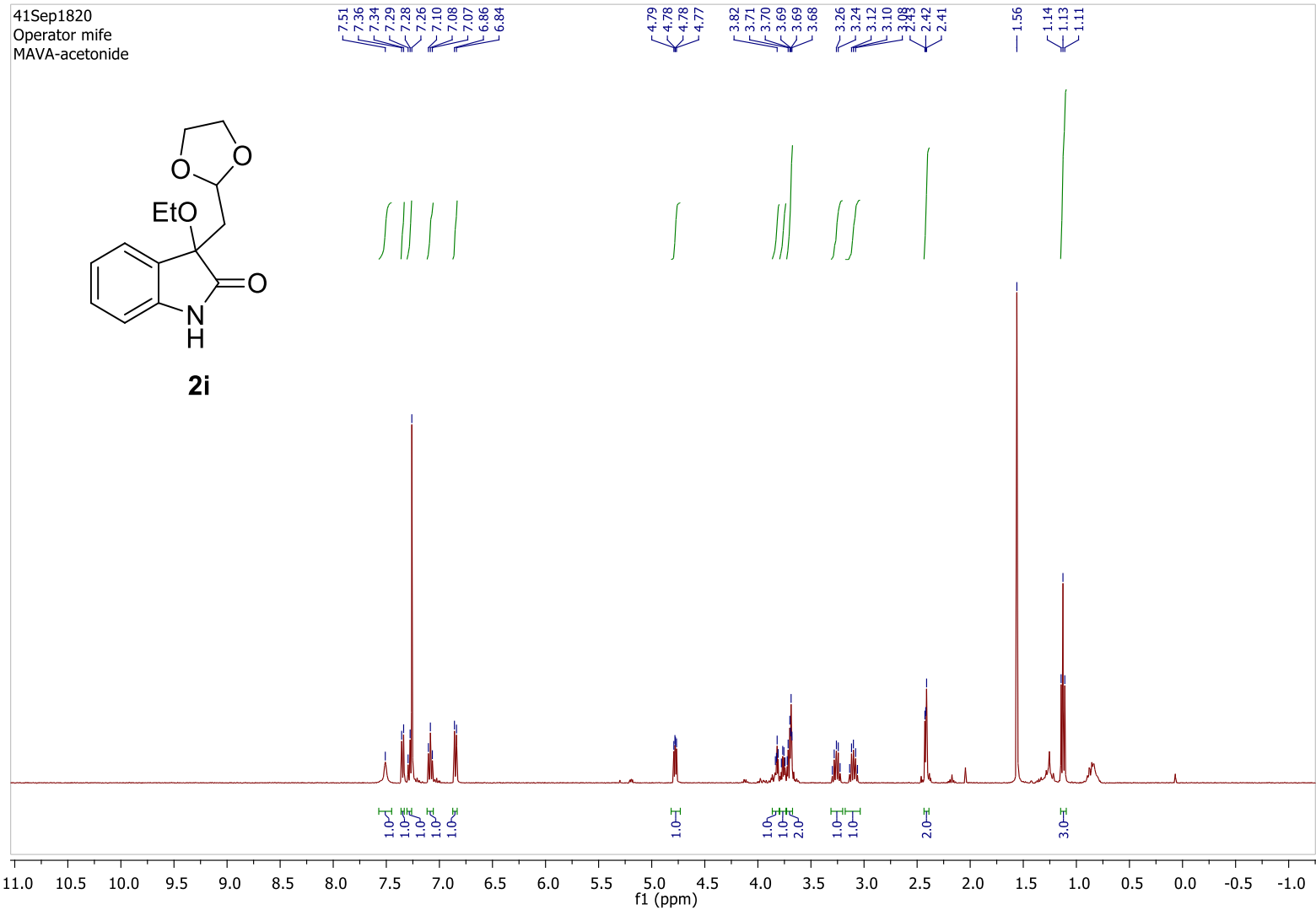

${ }^{1} \mathrm{H} \mathrm{NMR} \mathrm{in} \mathrm{CDCl}_{3}$ at $400 \mathrm{MHz}$

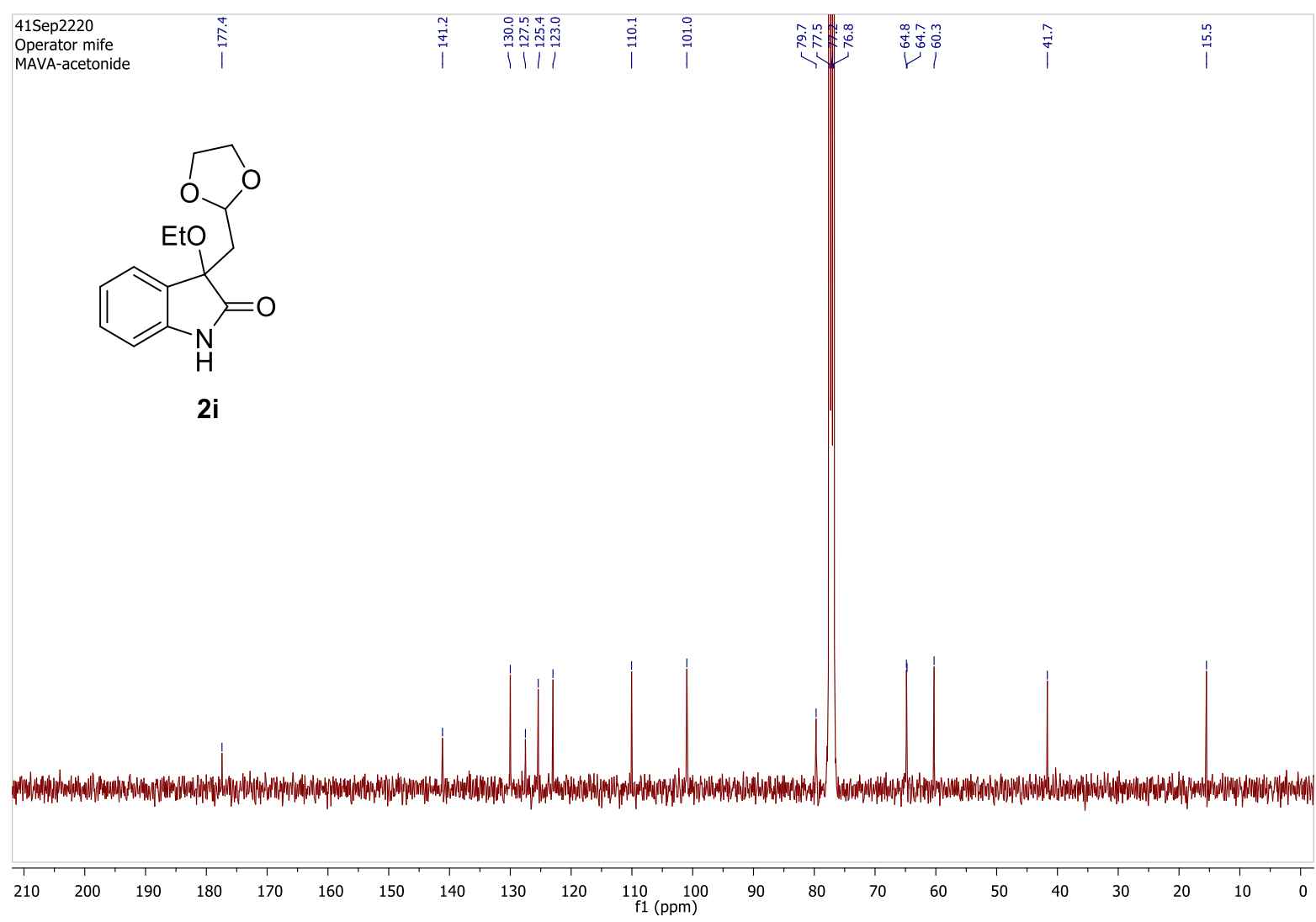

${ }^{13} \mathrm{C}\left\{{ }^{1} \mathrm{H}\right\}$ NMR in $\mathrm{CDCl}_{3}$ at $100 \mathrm{MHz}$ 


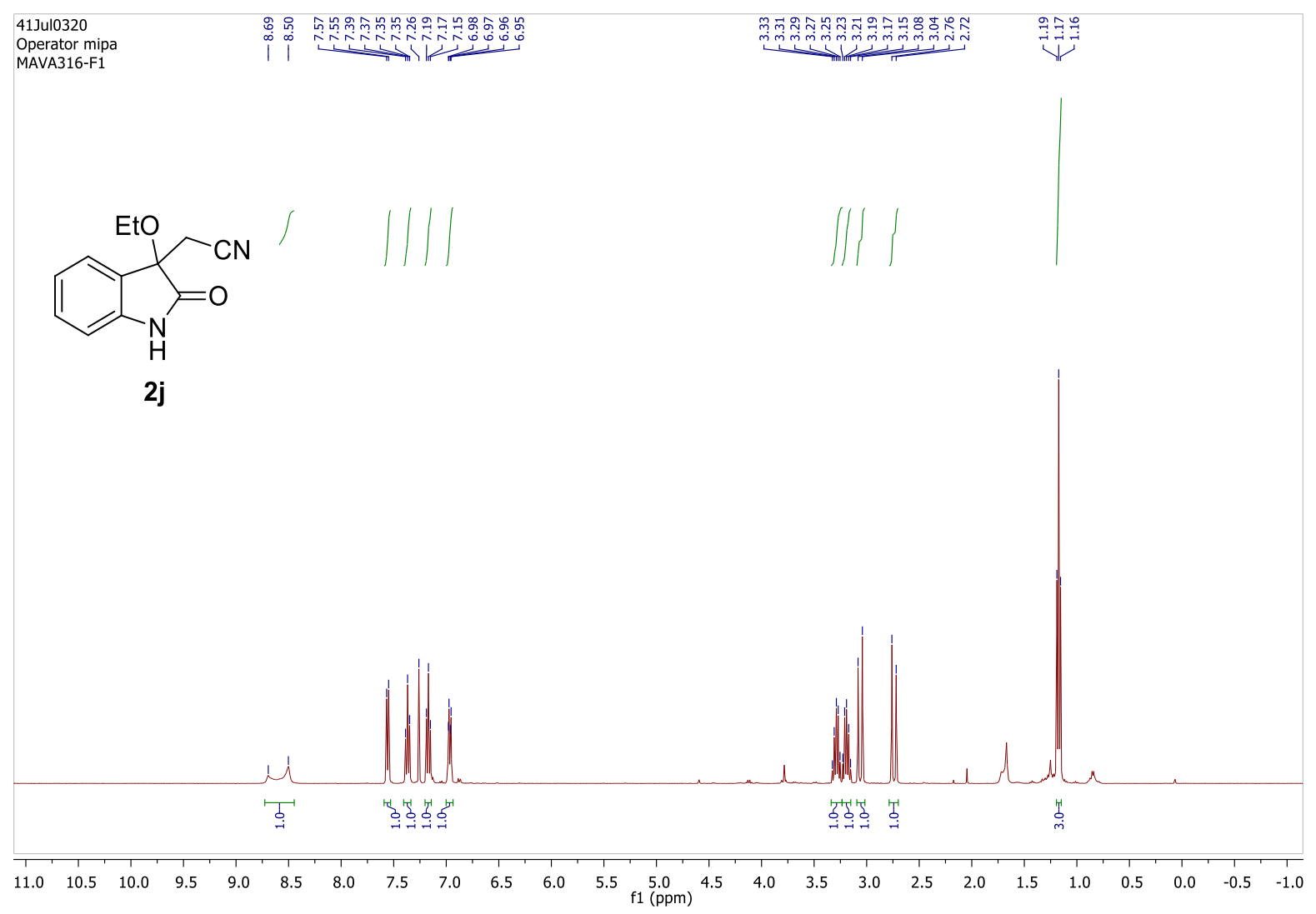

${ }^{1} \mathrm{H} \mathrm{NMR}$ in $\mathrm{CDCl}_{3}$ at $400 \mathrm{MHz}$

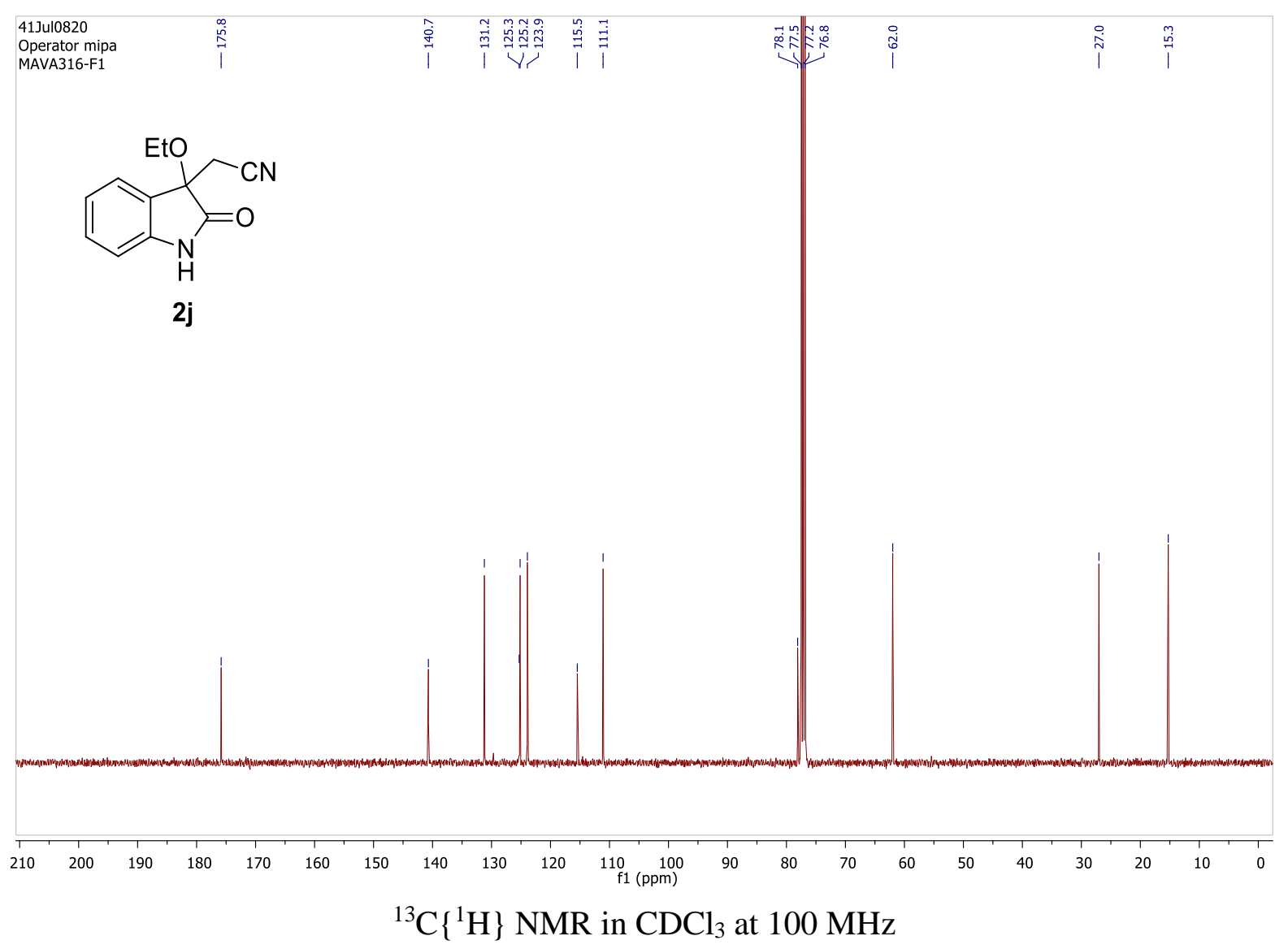




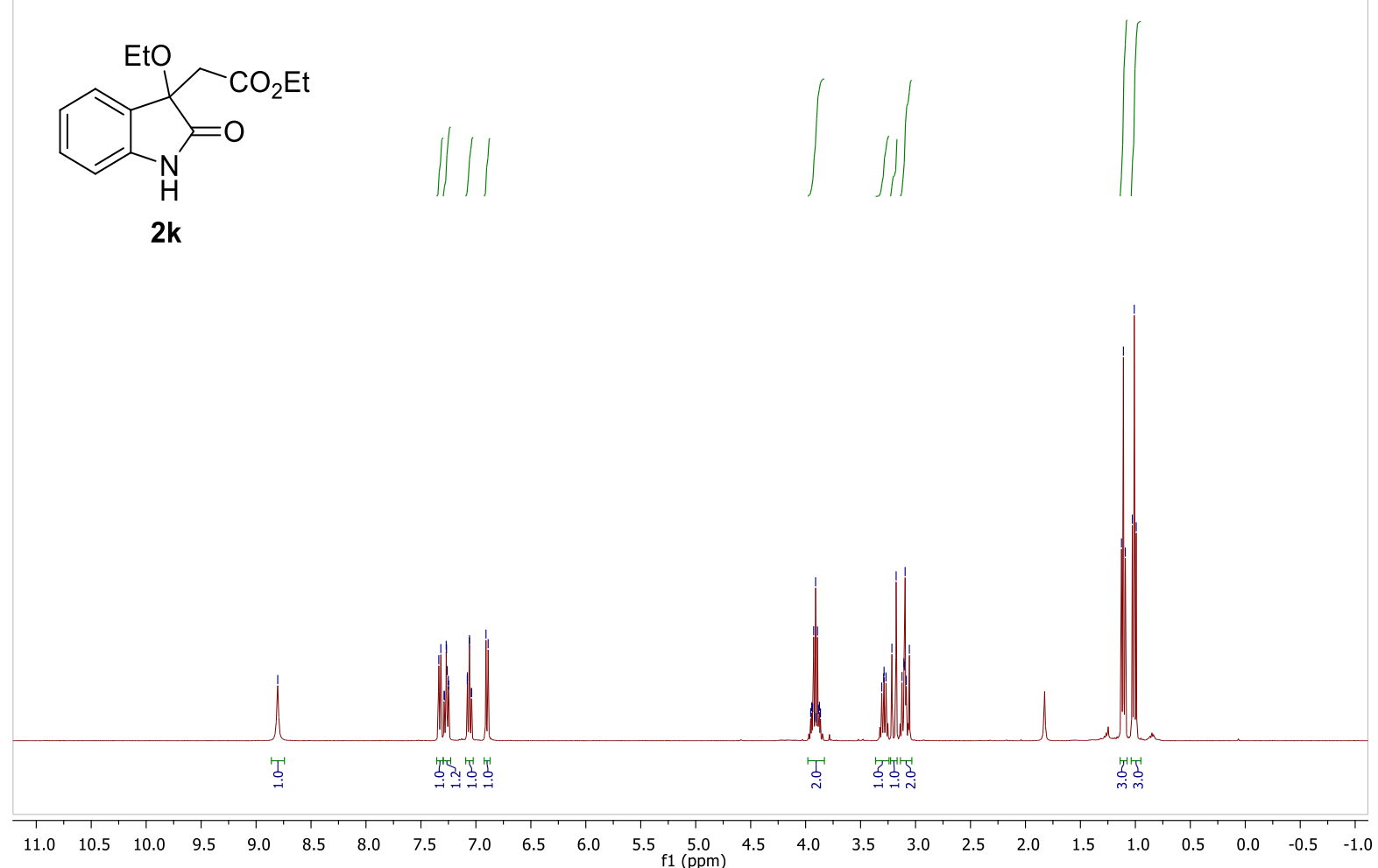

${ }^{1} \mathrm{H} \mathrm{NMR} \mathrm{in} \mathrm{CDCl}_{3}$ at $400 \mathrm{MHz}$

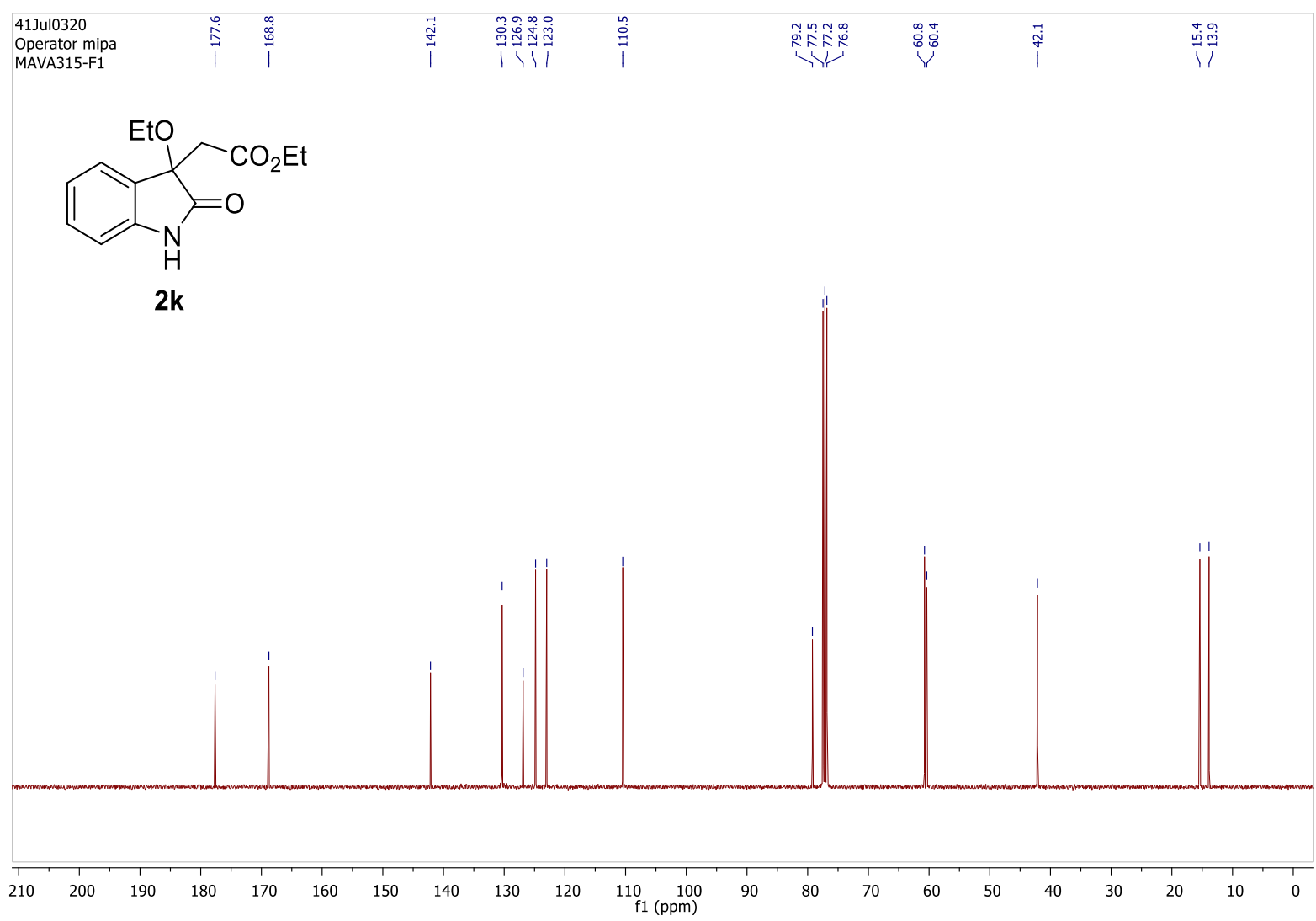

${ }^{13} \mathrm{C}\left\{{ }^{1} \mathrm{H}\right\} \mathrm{NMR}$ in $\mathrm{CDCl}_{3}$ at $100 \mathrm{MHz}$ 


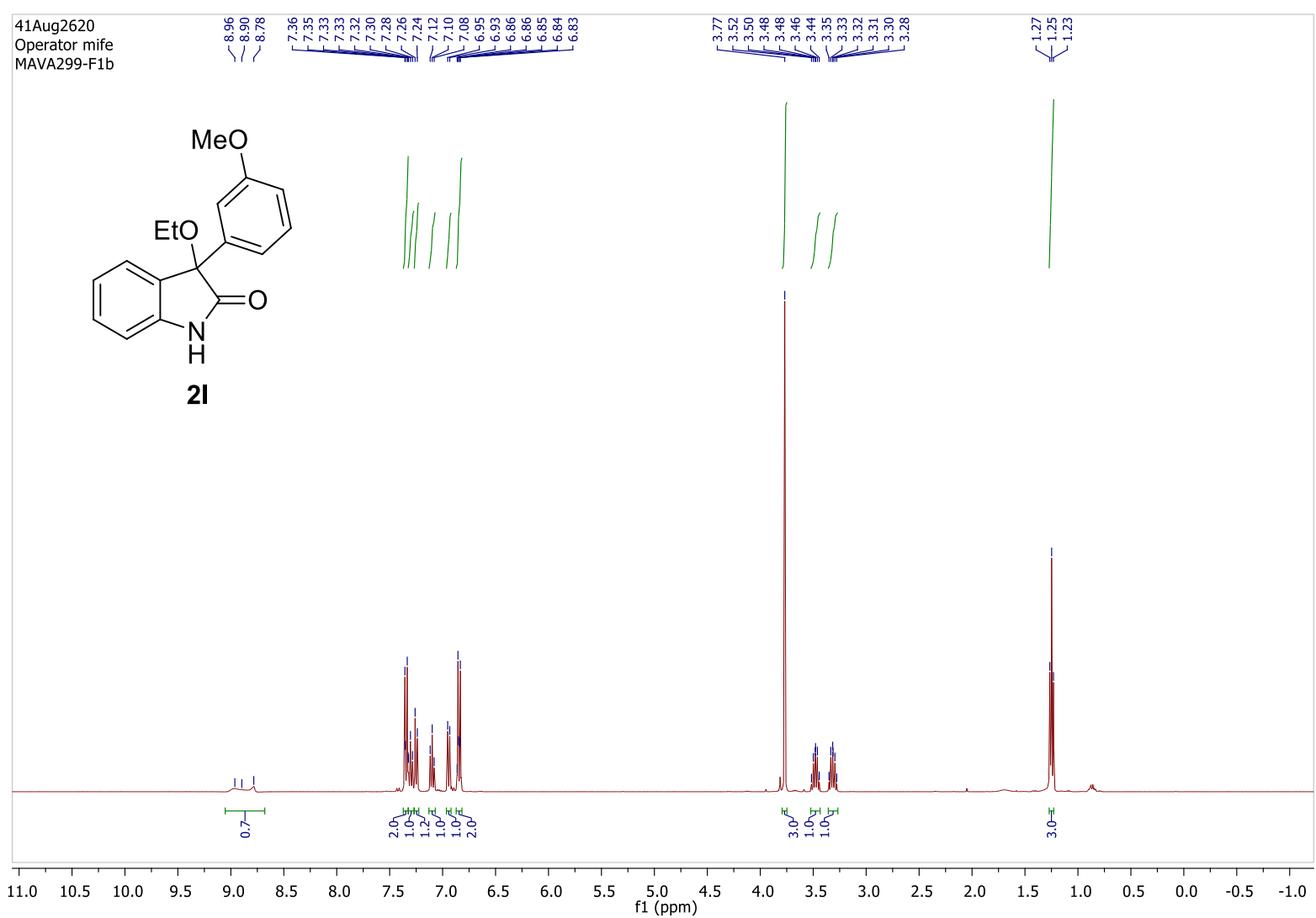

${ }^{1} \mathrm{H} \mathrm{NMR}$ in $\mathrm{CDCl}_{3}$ at $400 \mathrm{MHz}$

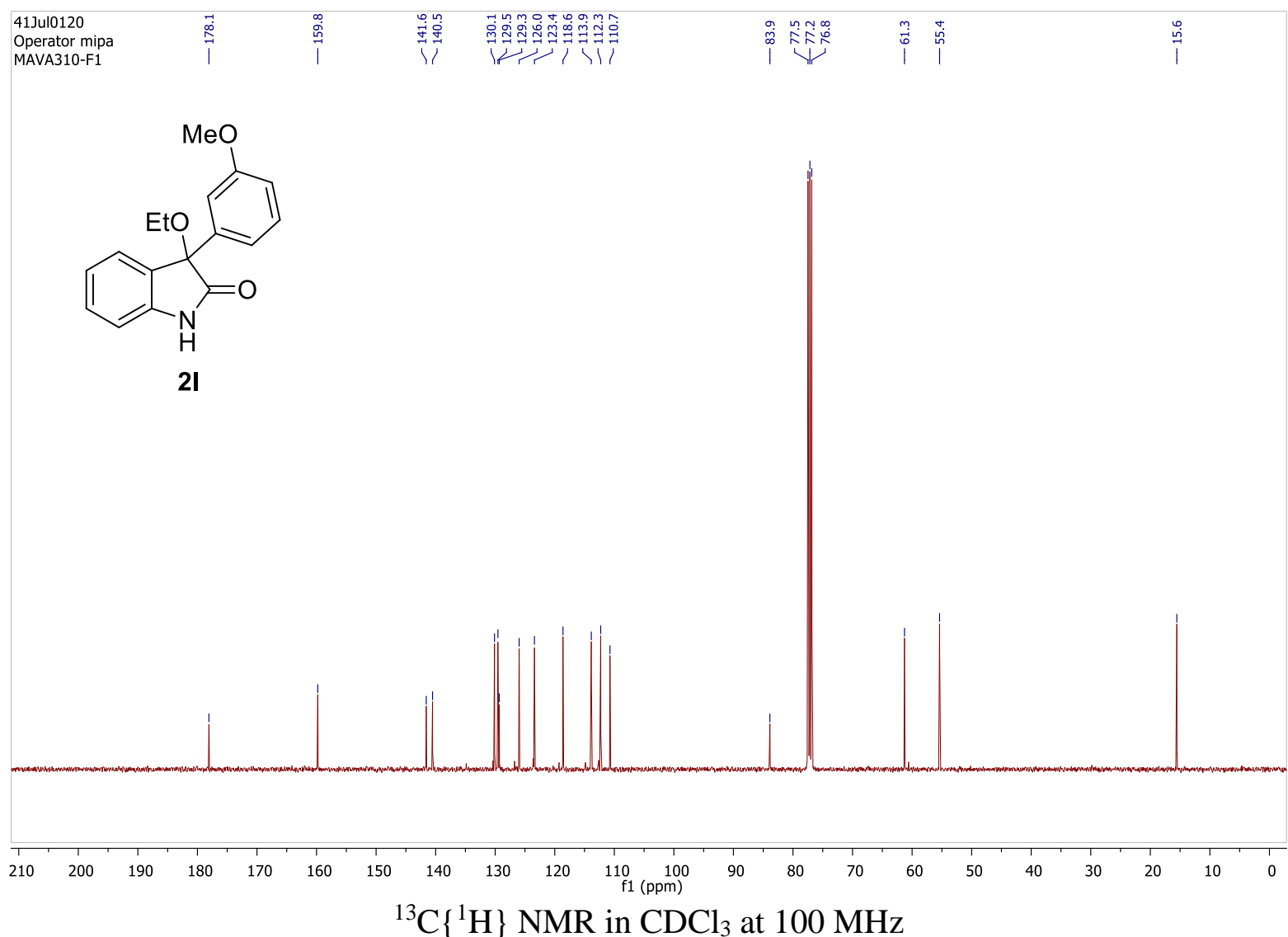




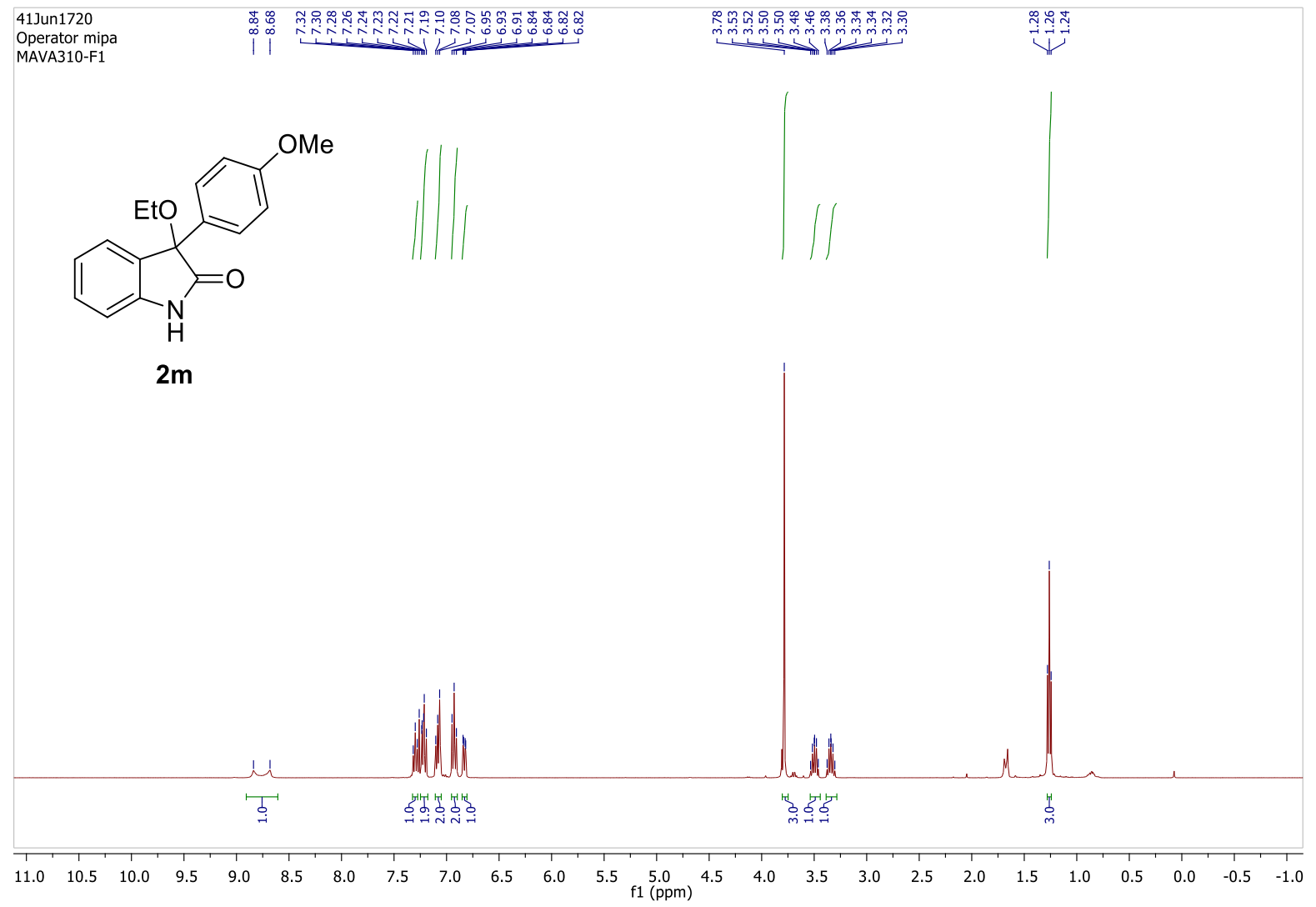

${ }^{1} \mathrm{H} \mathrm{NMR} \mathrm{in} \mathrm{CDCl}_{3}$ at $400 \mathrm{MHz}$

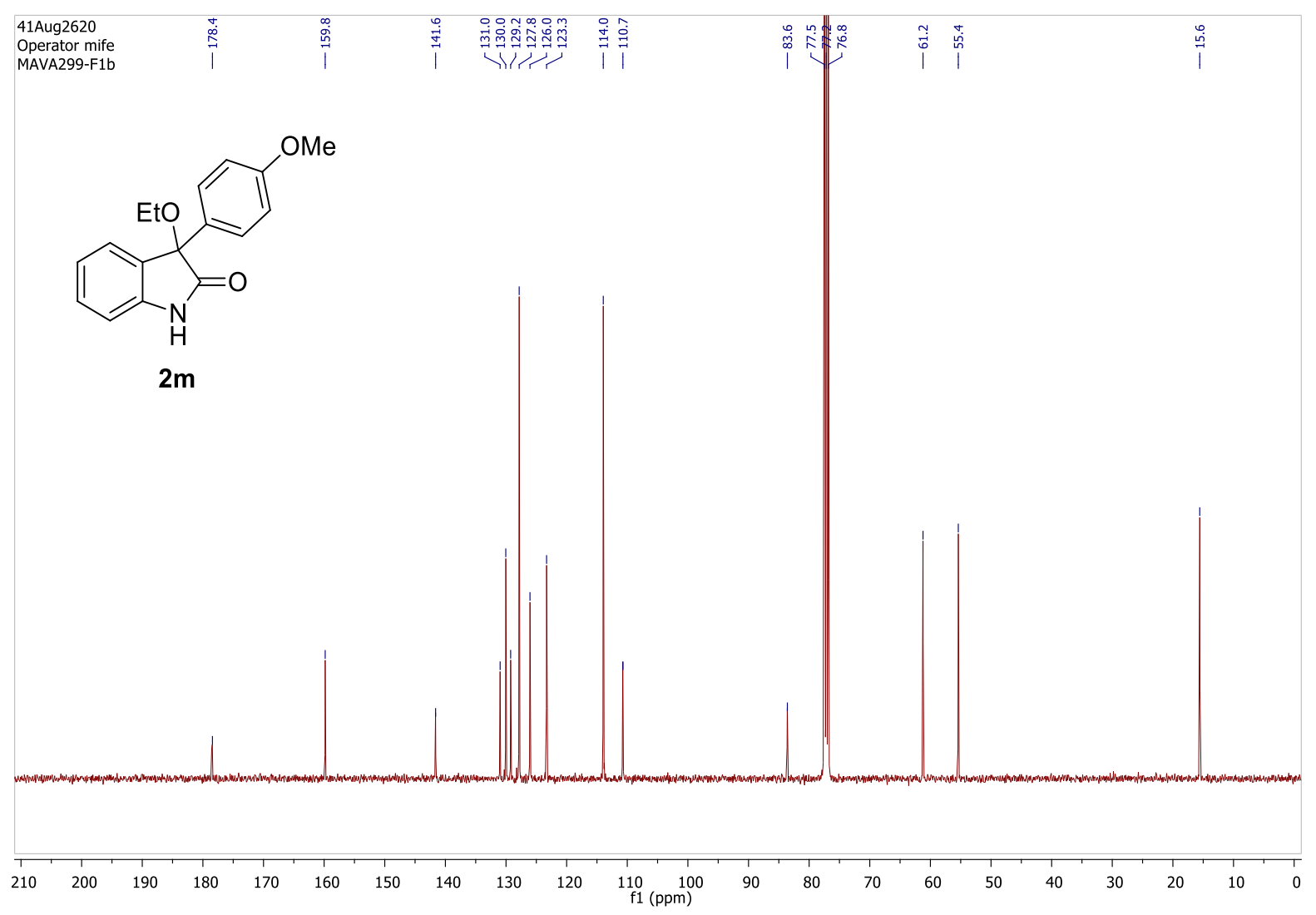

${ }^{13} \mathrm{C}\left\{{ }^{1} \mathrm{H}\right\} \mathrm{NMR}$ in $\mathrm{CDCl}_{3}$ at $100 \mathrm{MHz}$ 


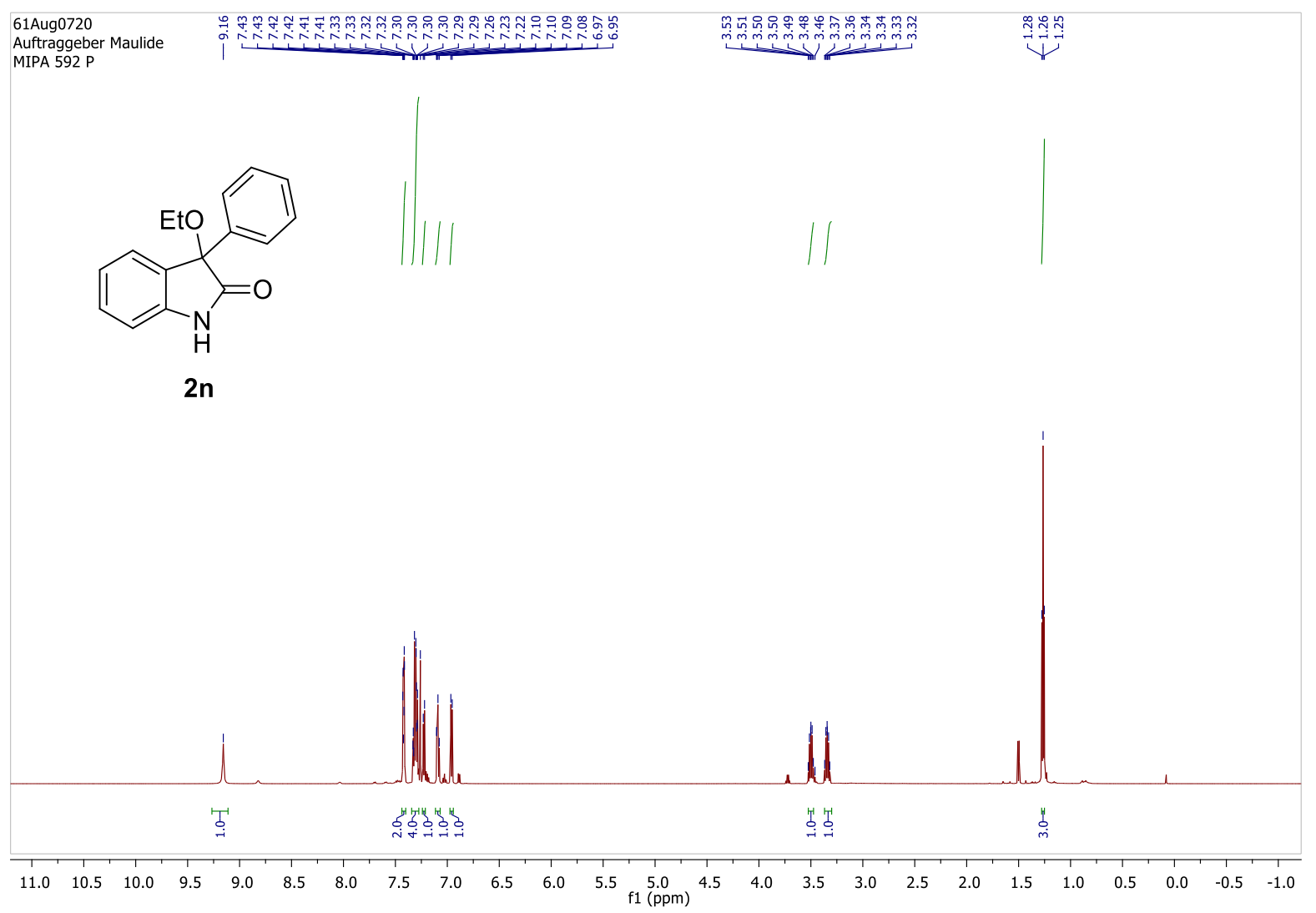

${ }^{1} \mathrm{H} \mathrm{NMR}$ in $\mathrm{CDCl}_{3}$ at $600 \mathrm{MHz}$

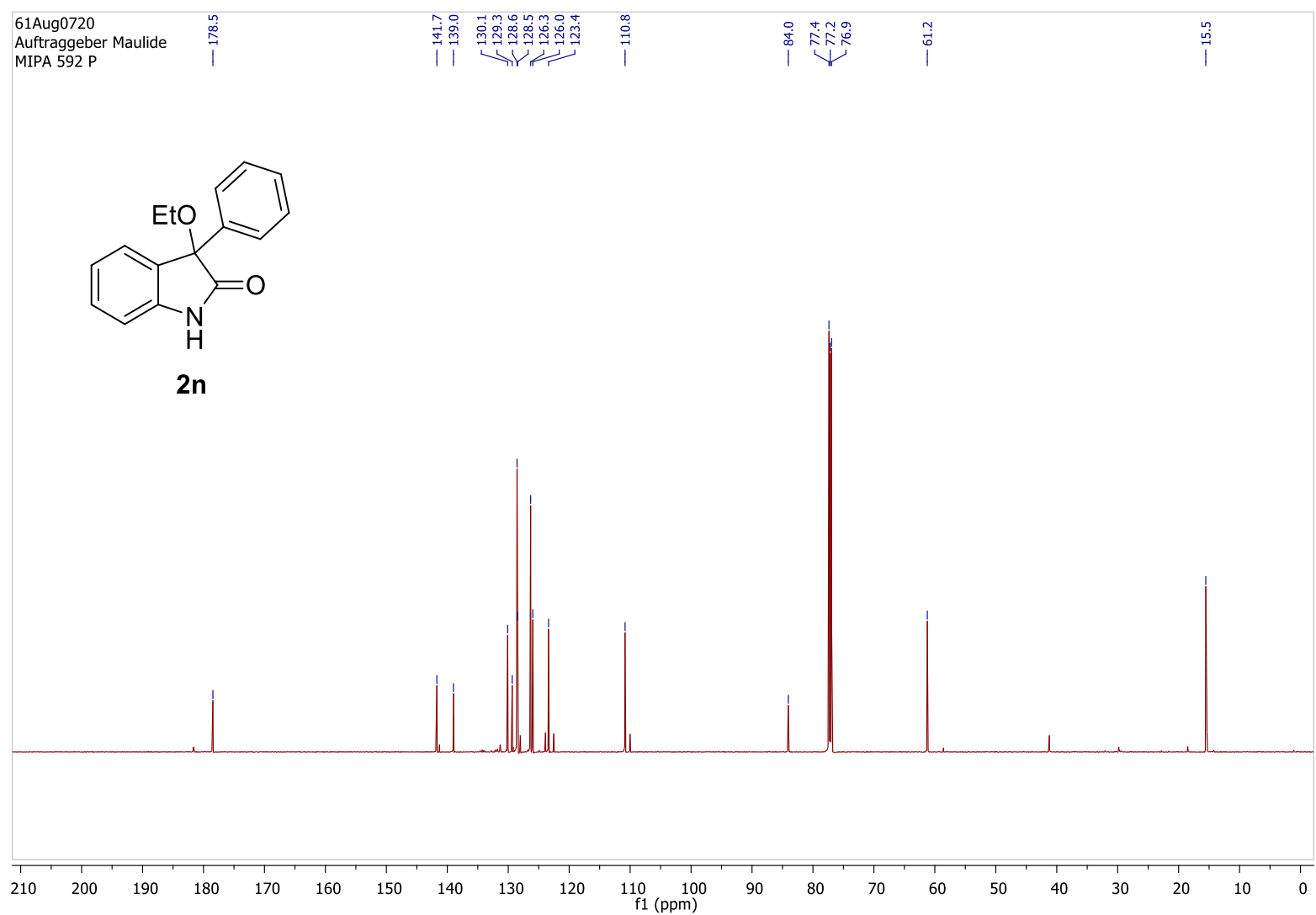

${ }^{13} \mathrm{C}\left\{{ }^{1} \mathrm{H}\right\} \mathrm{NMR}$ in $\mathrm{CDCl}_{3}$ at $150 \mathrm{MHz}$ 


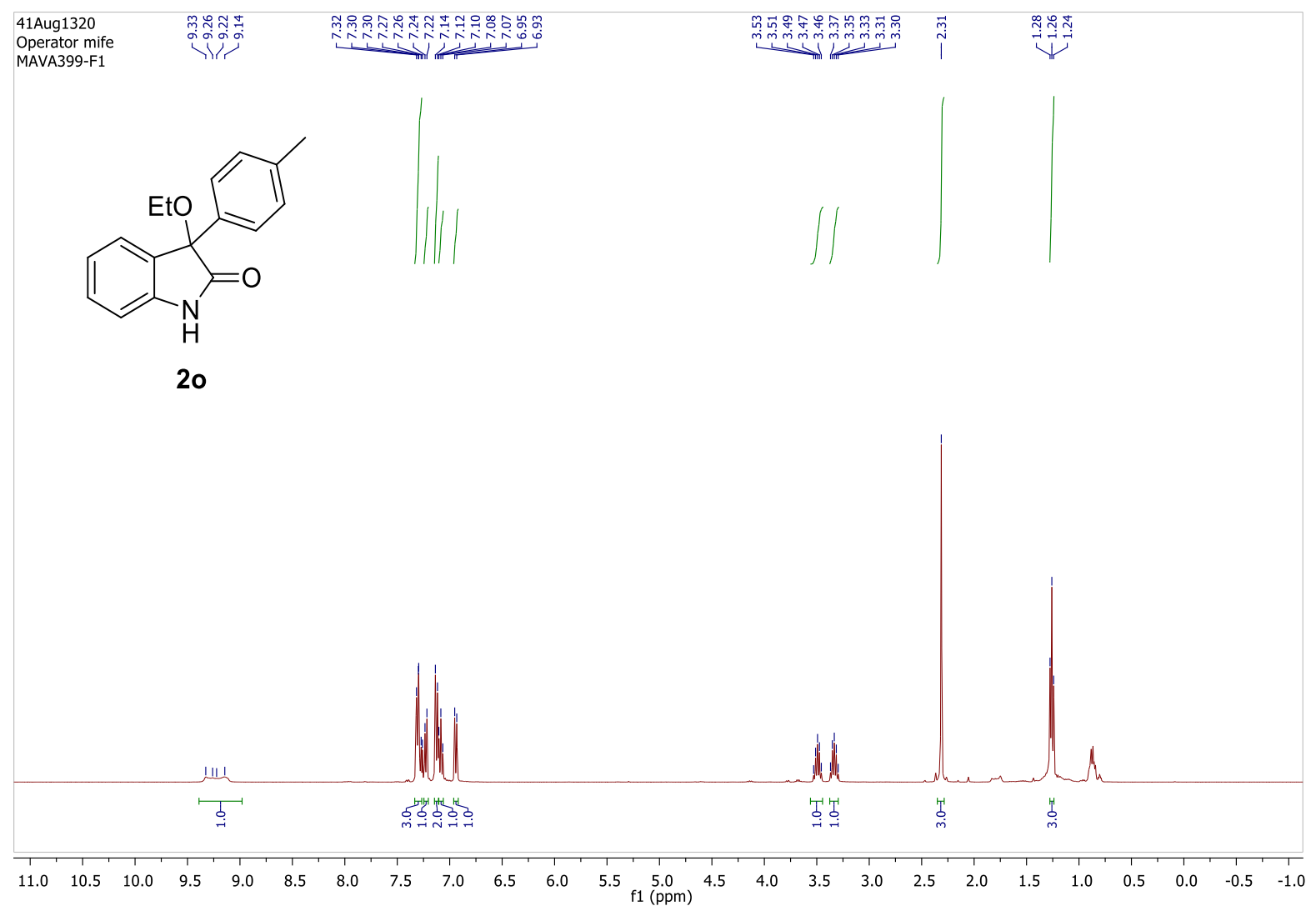

${ }^{1} \mathrm{H} \mathrm{NMR}$ in $\mathrm{CDCl}_{3}$ at $400 \mathrm{MHz}$

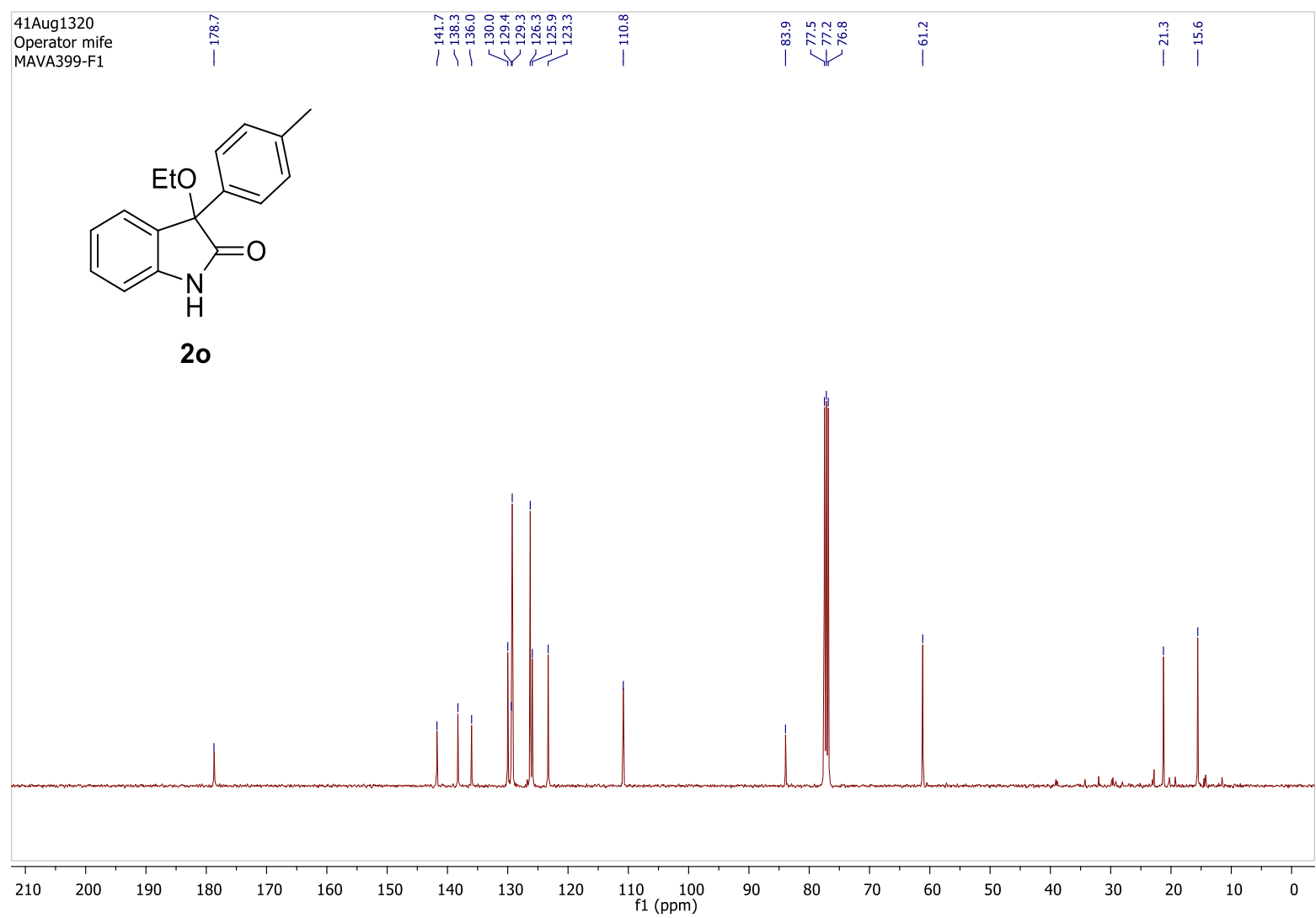

${ }^{13} \mathrm{C}\left\{{ }^{1} \mathrm{H}\right\} \mathrm{NMR}$ in $\mathrm{CDCl}_{3}$ at $100 \mathrm{MH}$ 


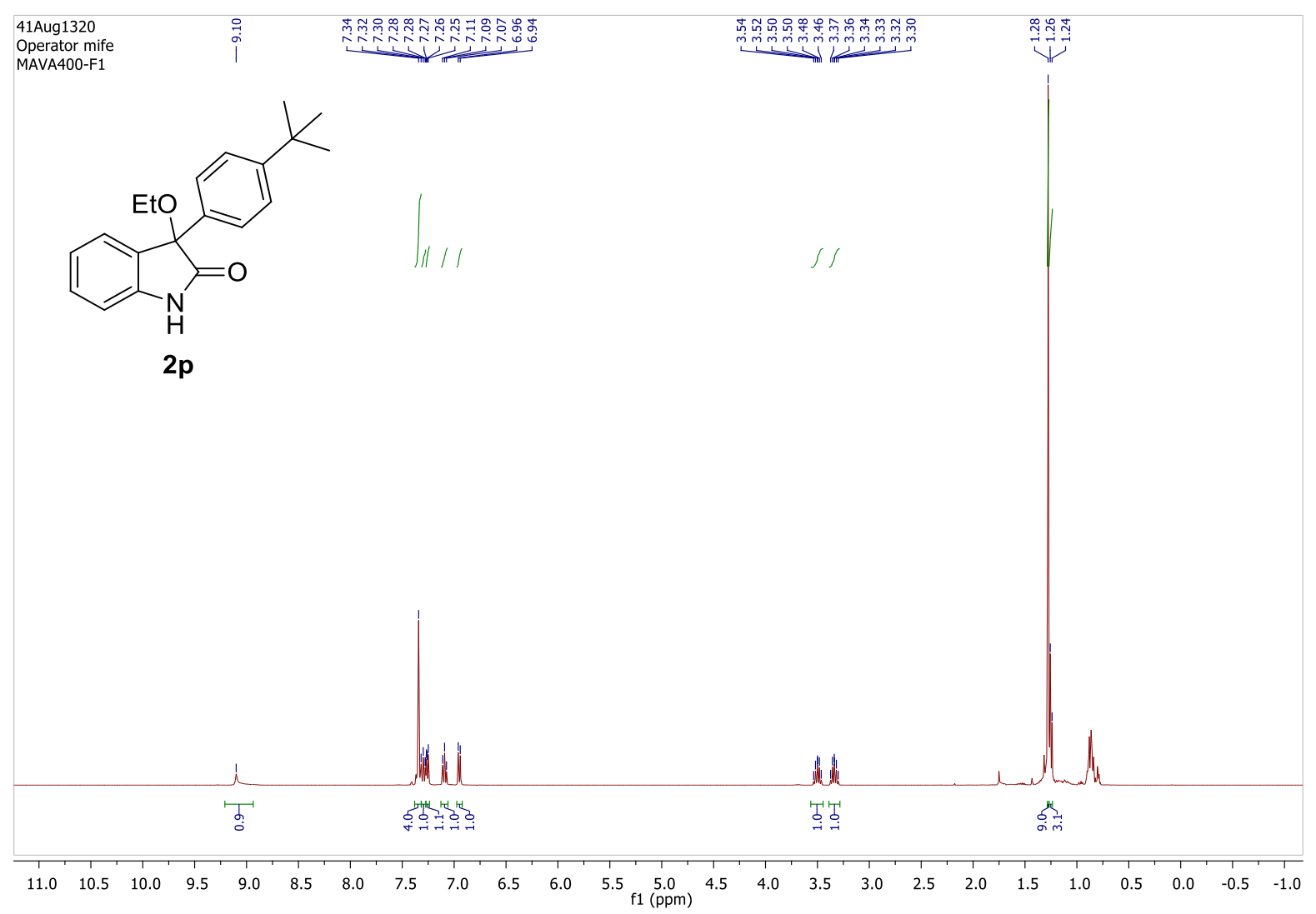

${ }^{1} \mathrm{H} \mathrm{NMR}$ in $\mathrm{CDCl}_{3}$ at $400 \mathrm{MHz}$

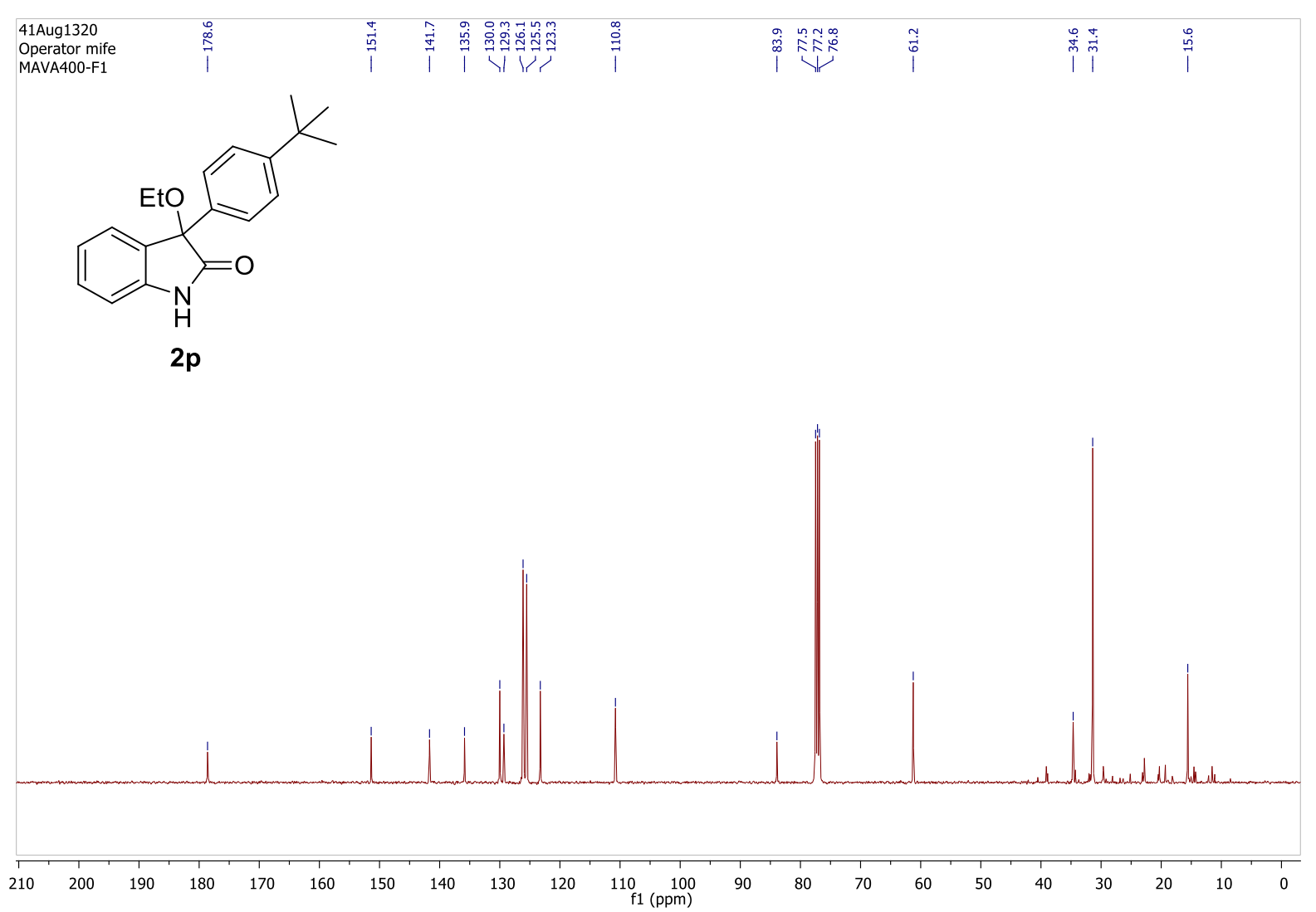

${ }^{13} \mathrm{C}\left\{{ }^{1} \mathrm{H}\right\} \mathrm{NMR}$ in $\mathrm{CDCl}_{3}$ at $100 \mathrm{MHz}$ 
<smiles>CCOC1(c2ccc(-c3ccccc3)cc2)C(=O)Nc2ccccc21</smiles>

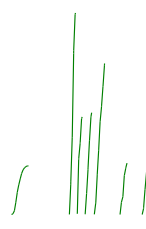

$2 q$

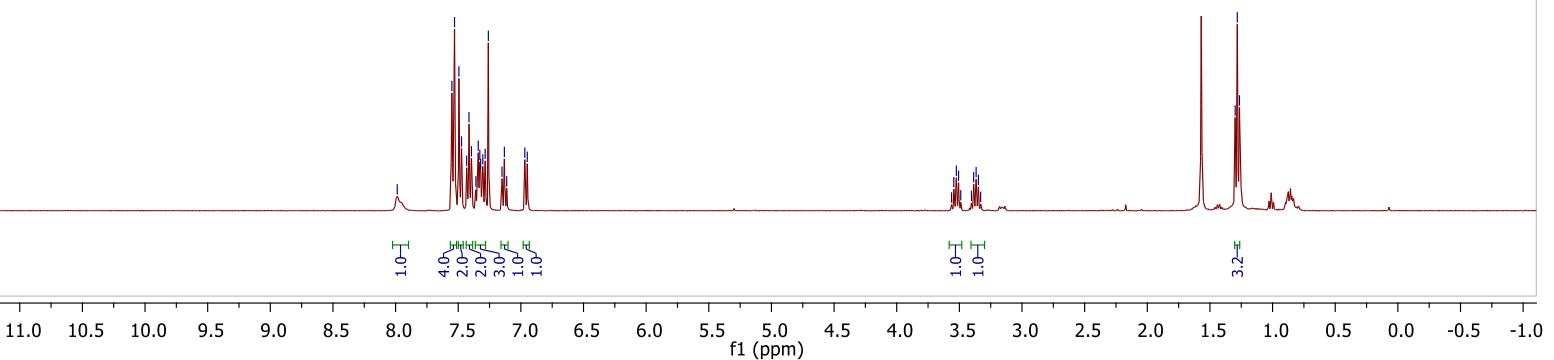

${ }^{1} \mathrm{H} \mathrm{NMR}$ in $\mathrm{CDCl}_{3}$ at $400 \mathrm{MHz}$

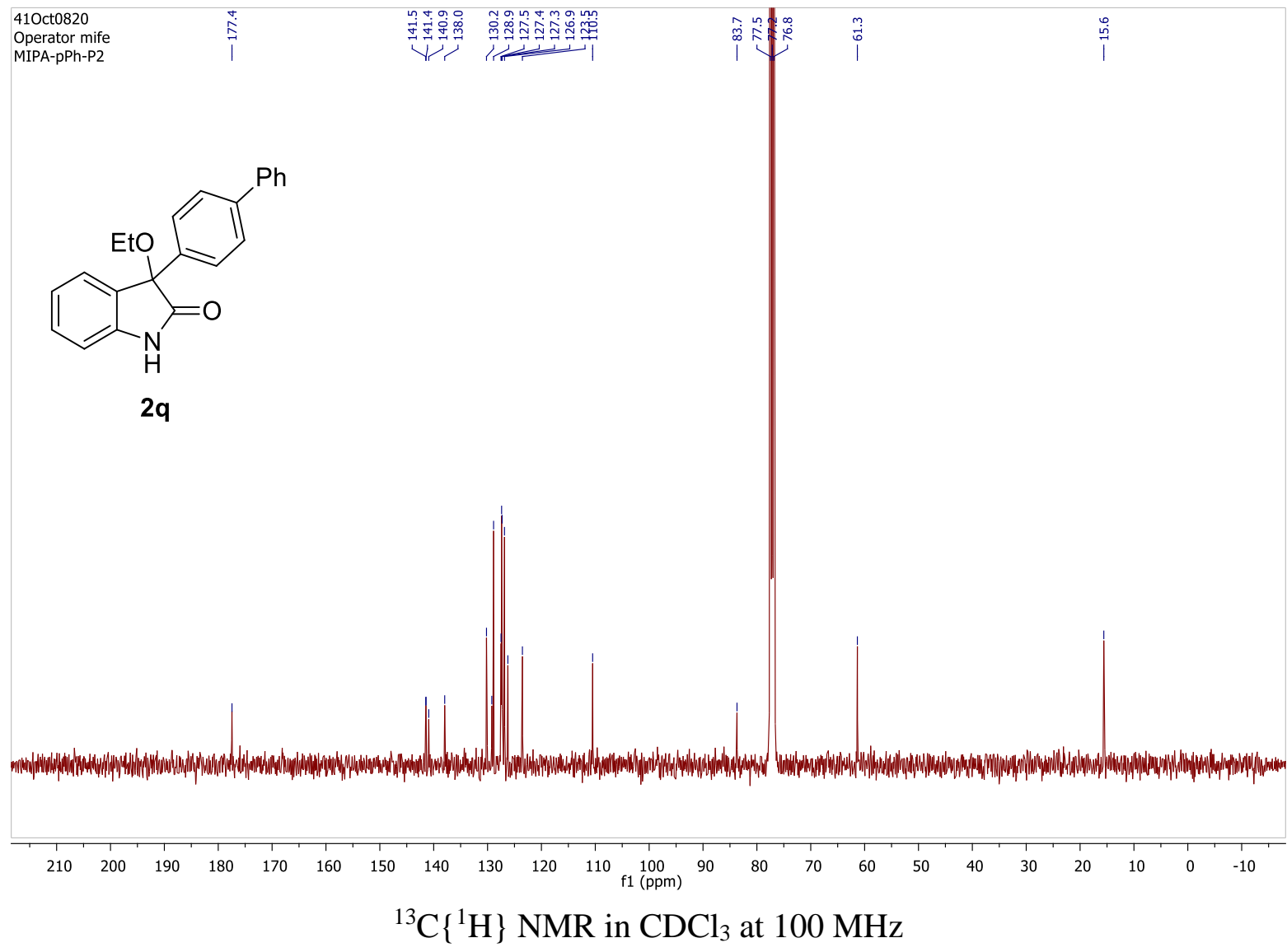




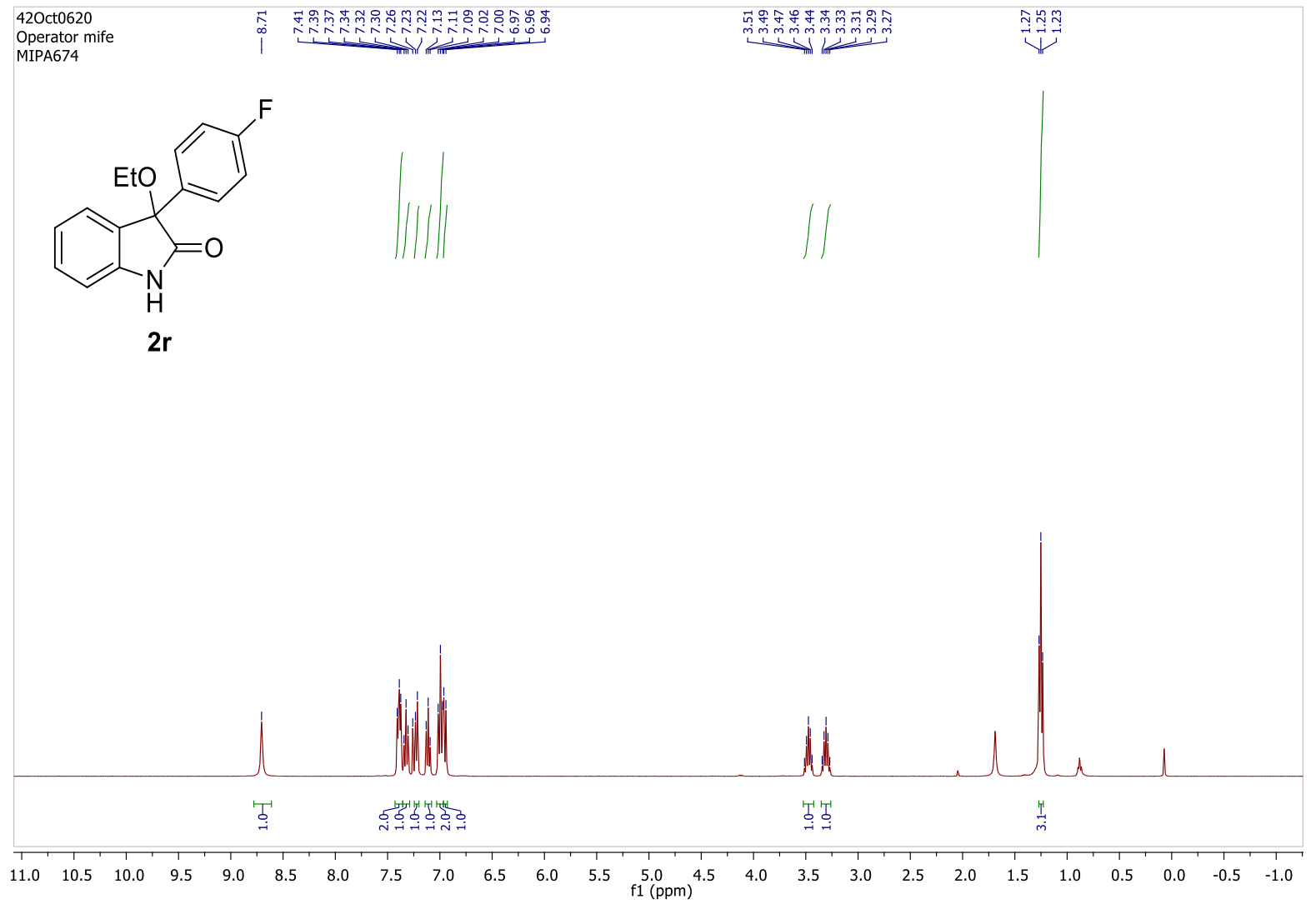

${ }^{1} \mathrm{H} \mathrm{NMR}$ in $\mathrm{CDCl}_{3}$ at $600 \mathrm{MHz}$

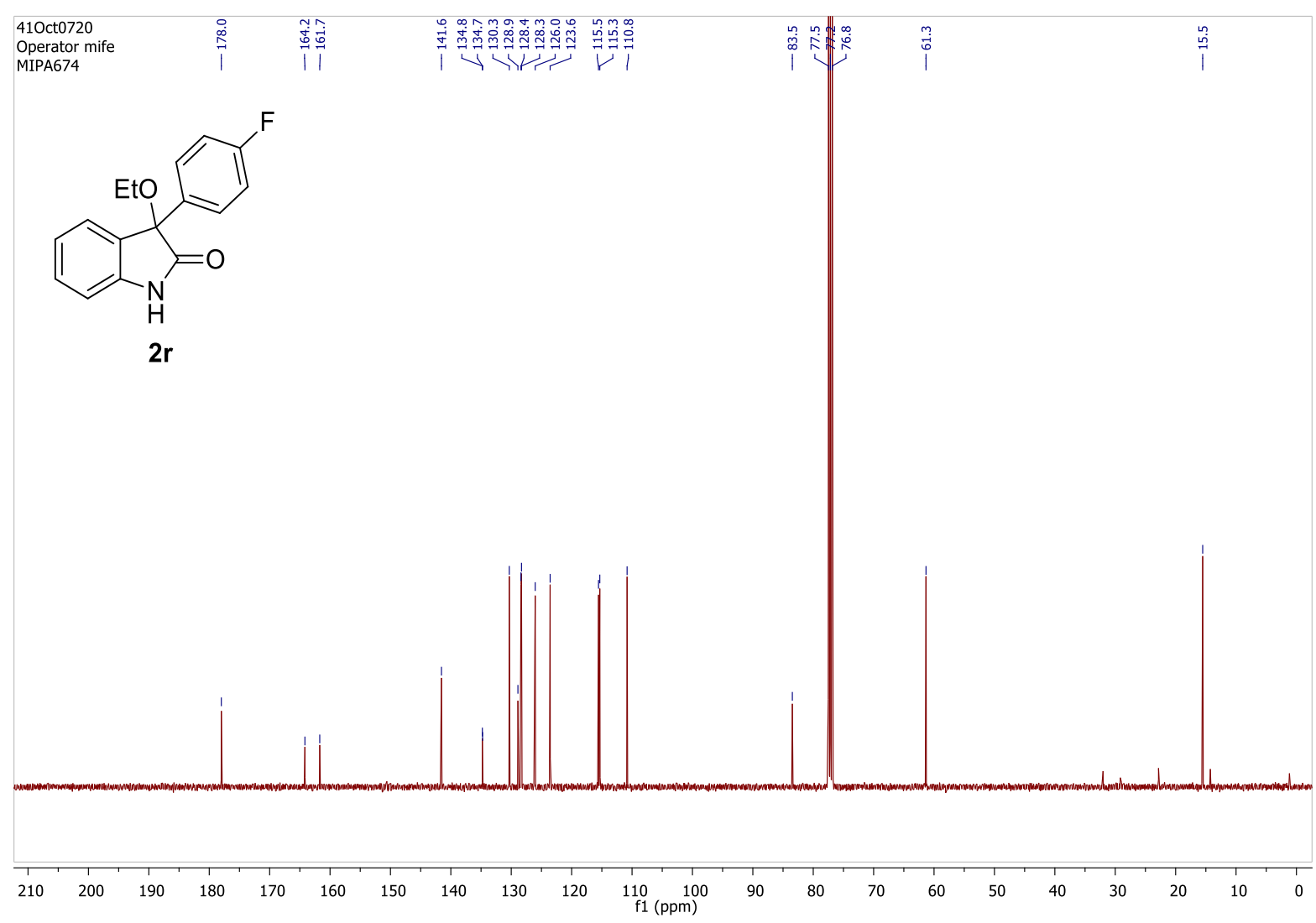

${ }^{13} \mathrm{C}\left\{{ }^{1} \mathrm{H}\right\} \mathrm{NMR}$ in $\mathrm{CDCl}_{3}$ at $150 \mathrm{MHz}$ 

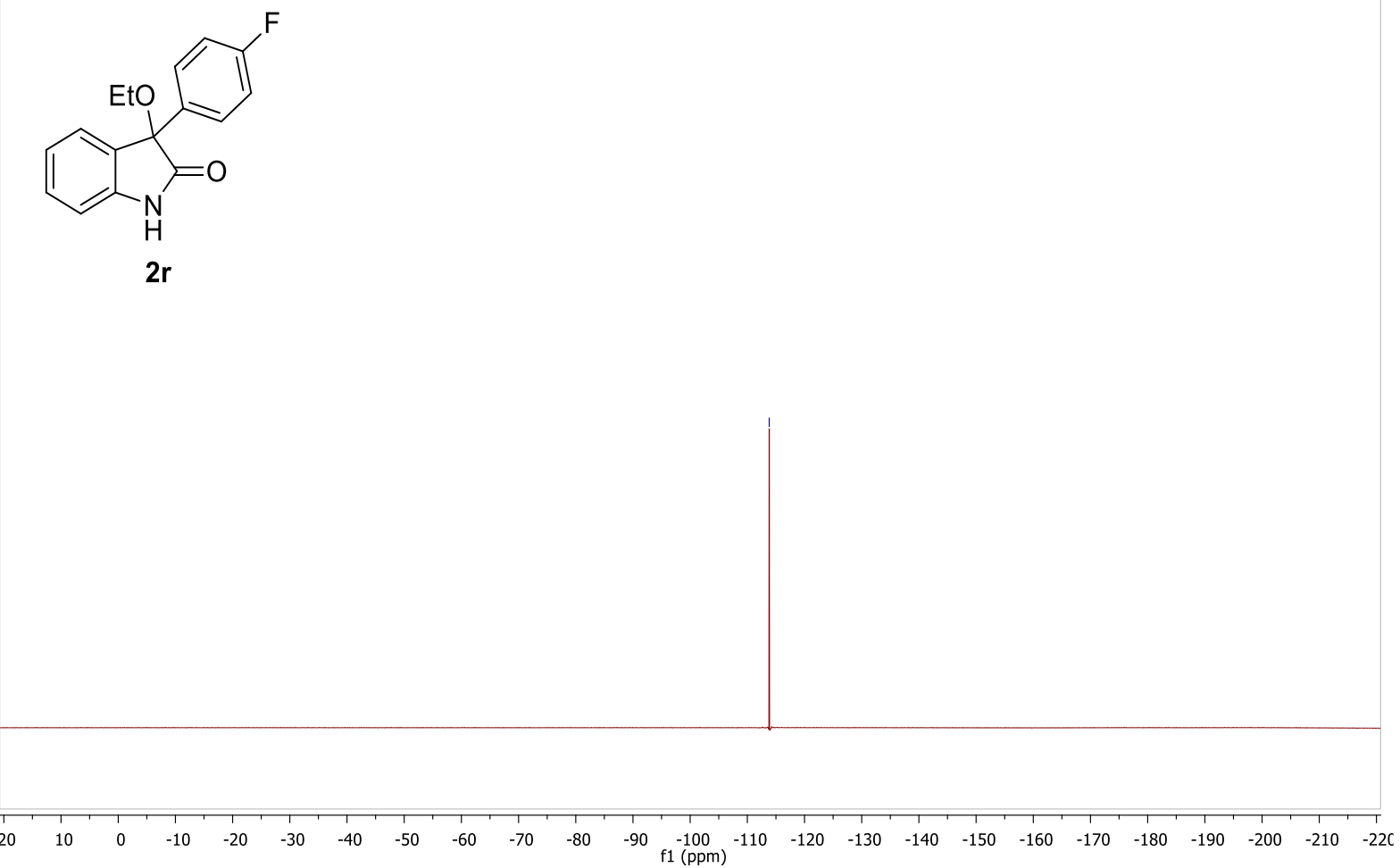

${ }^{19} \mathrm{~F} \mathrm{NMR}$ in $\mathrm{CDCl}_{3}$ at $376 \mathrm{MHz}$ 


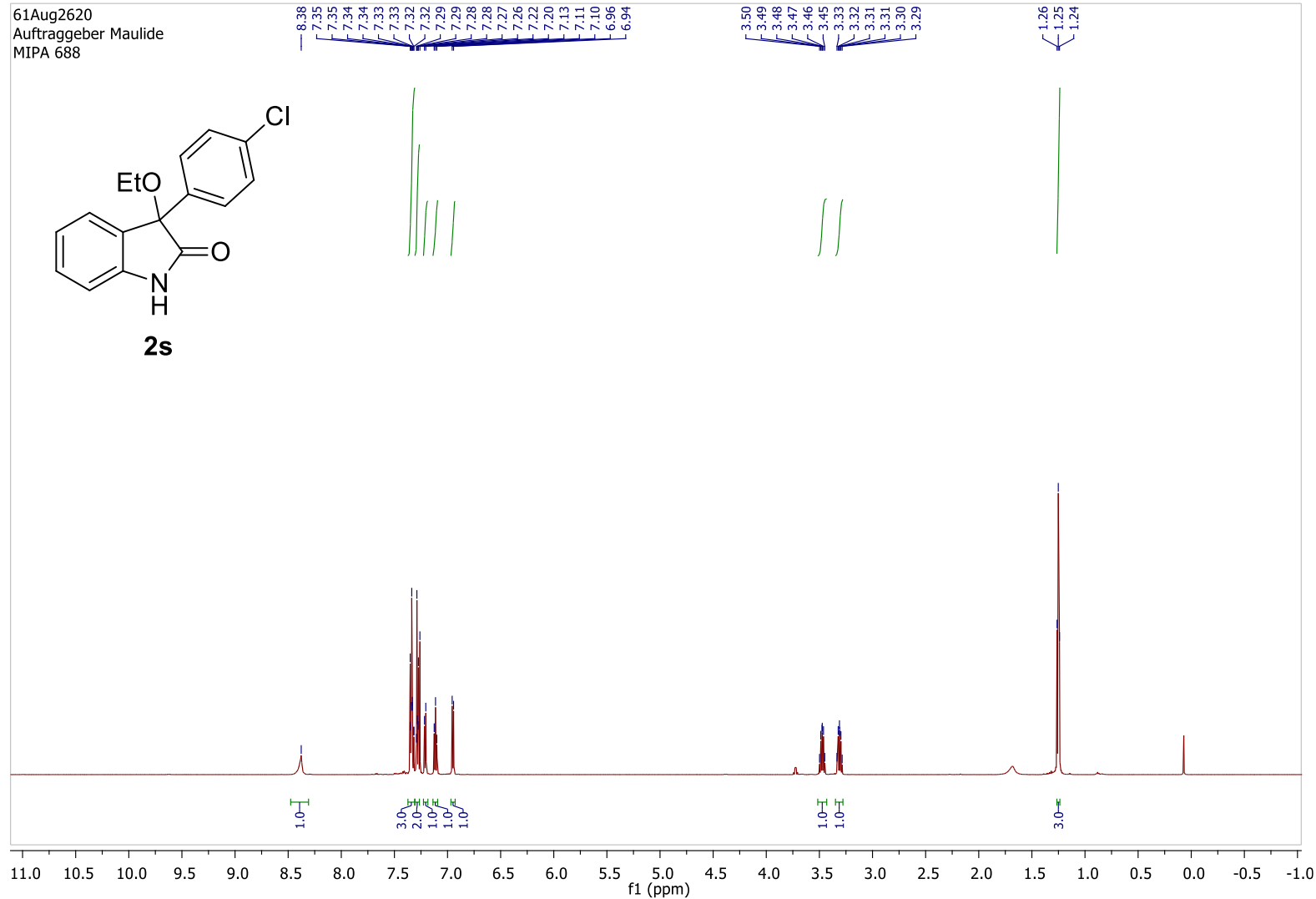

${ }^{1} \mathrm{H} \mathrm{NMR}$ in $\mathrm{CDCl}_{3}$ at $600 \mathrm{MHz}$

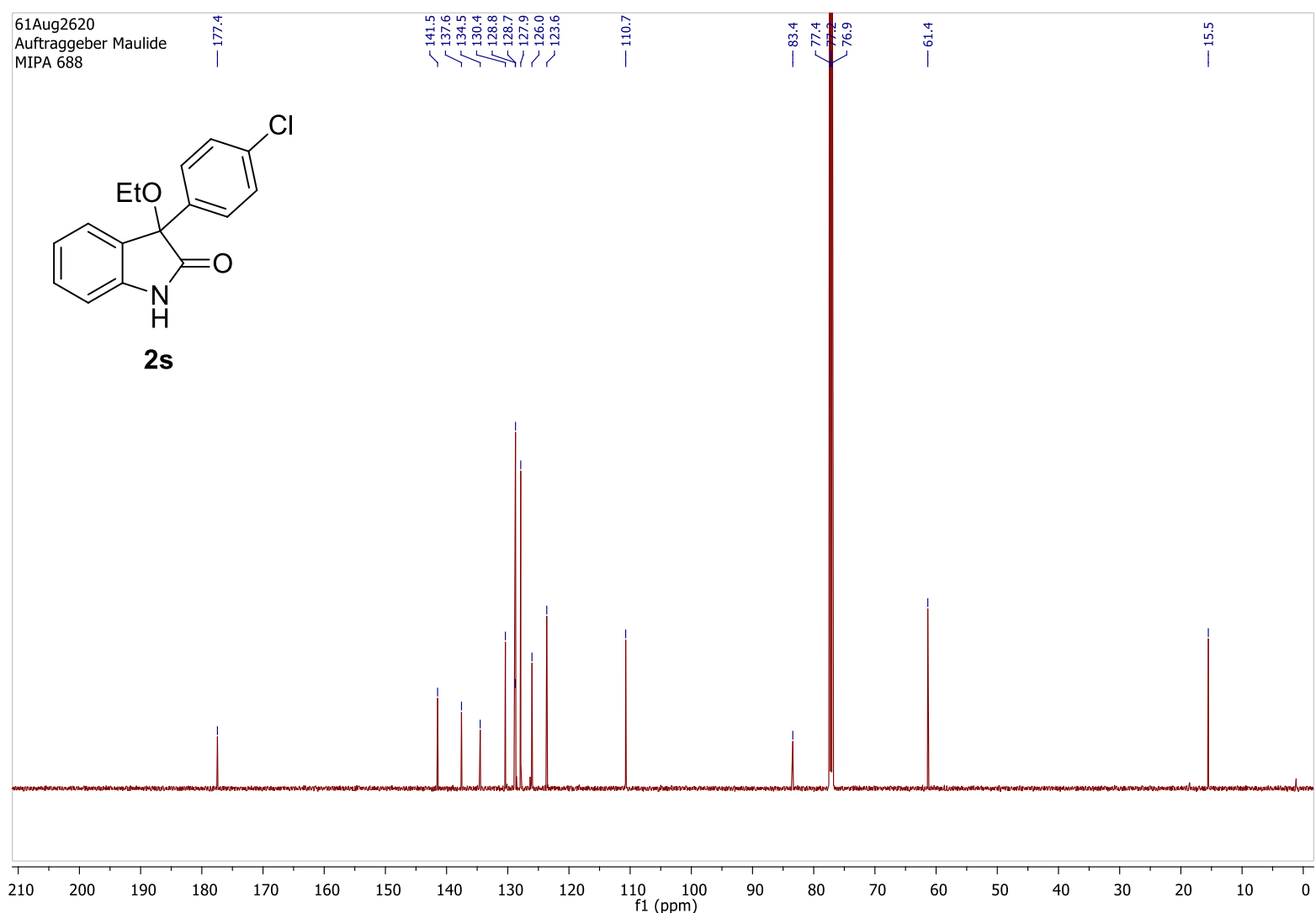

${ }^{13} \mathrm{C}\left\{{ }^{1} \mathrm{H}\right\} \mathrm{NMR}$ in $\mathrm{CDCl}_{3}$ at $150 \mathrm{MHz}$ 


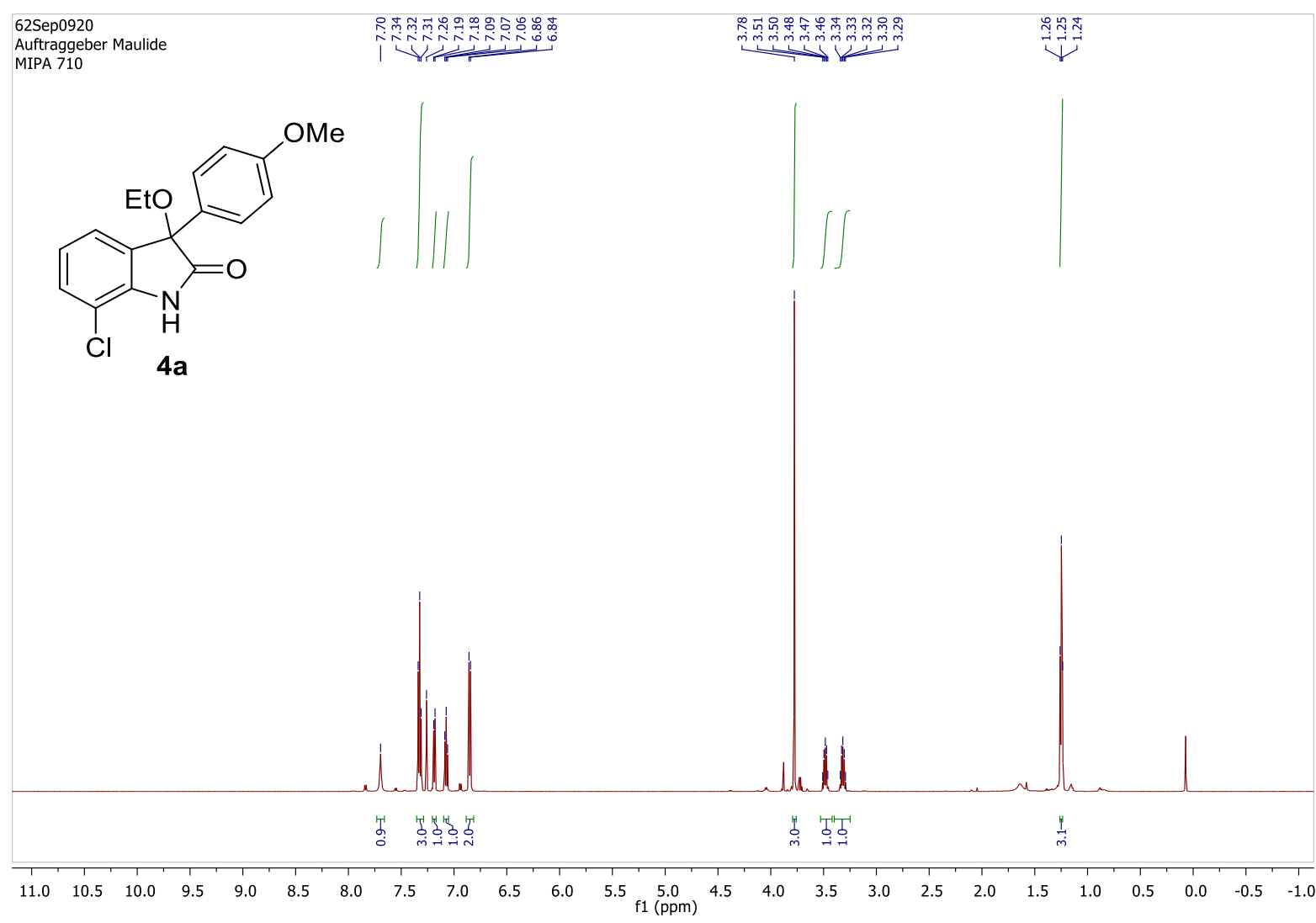

${ }^{1} \mathrm{H} \mathrm{NMR} \mathrm{in} \mathrm{CDCl}_{3}$ at $600 \mathrm{MHz}$

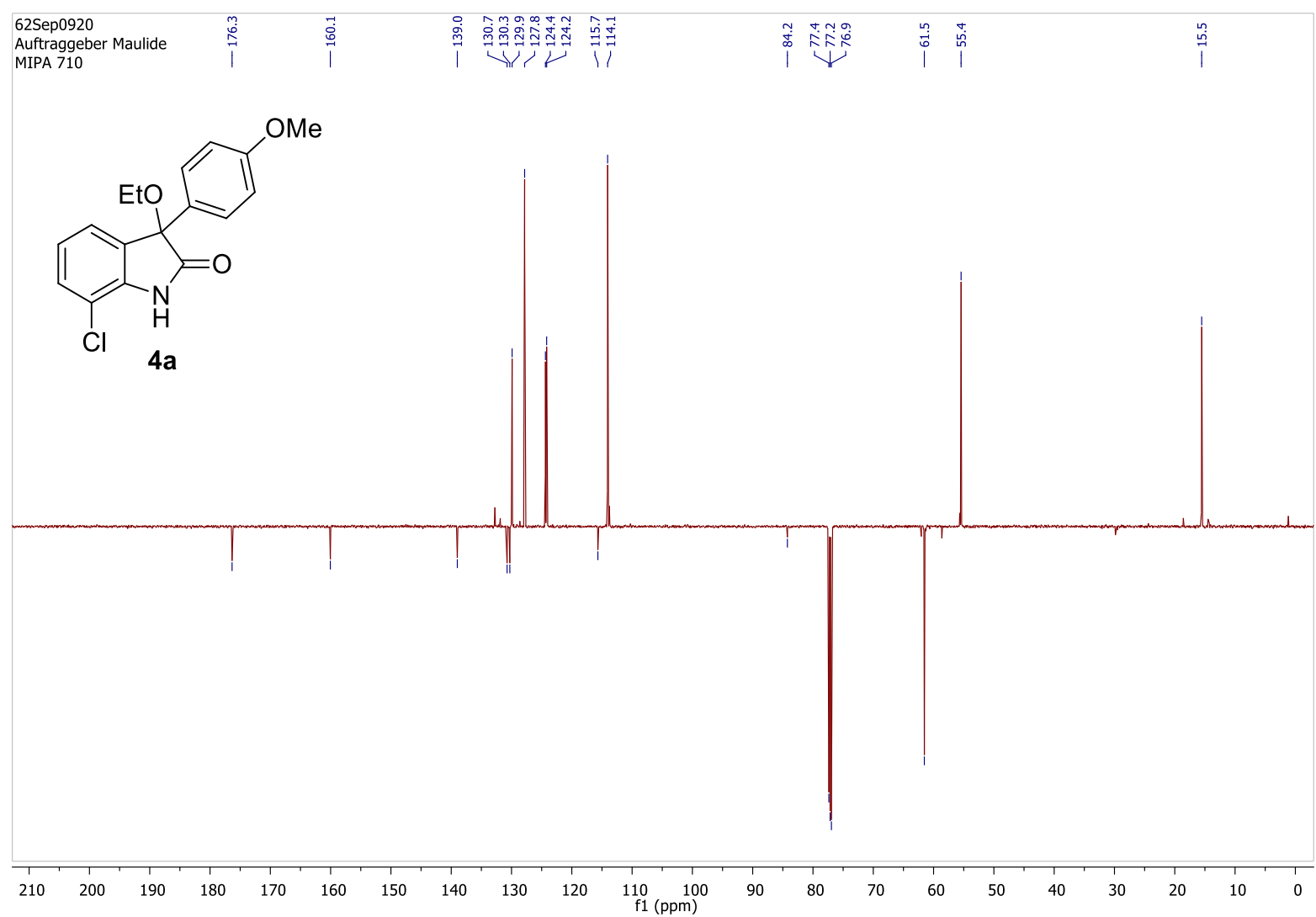

${ }^{13} \mathrm{C}\left\{{ }^{1} \mathrm{H}\right\} \mathrm{NMR}$ in $\mathrm{CDCl}_{3}$ at $150 \mathrm{MHz}$ 


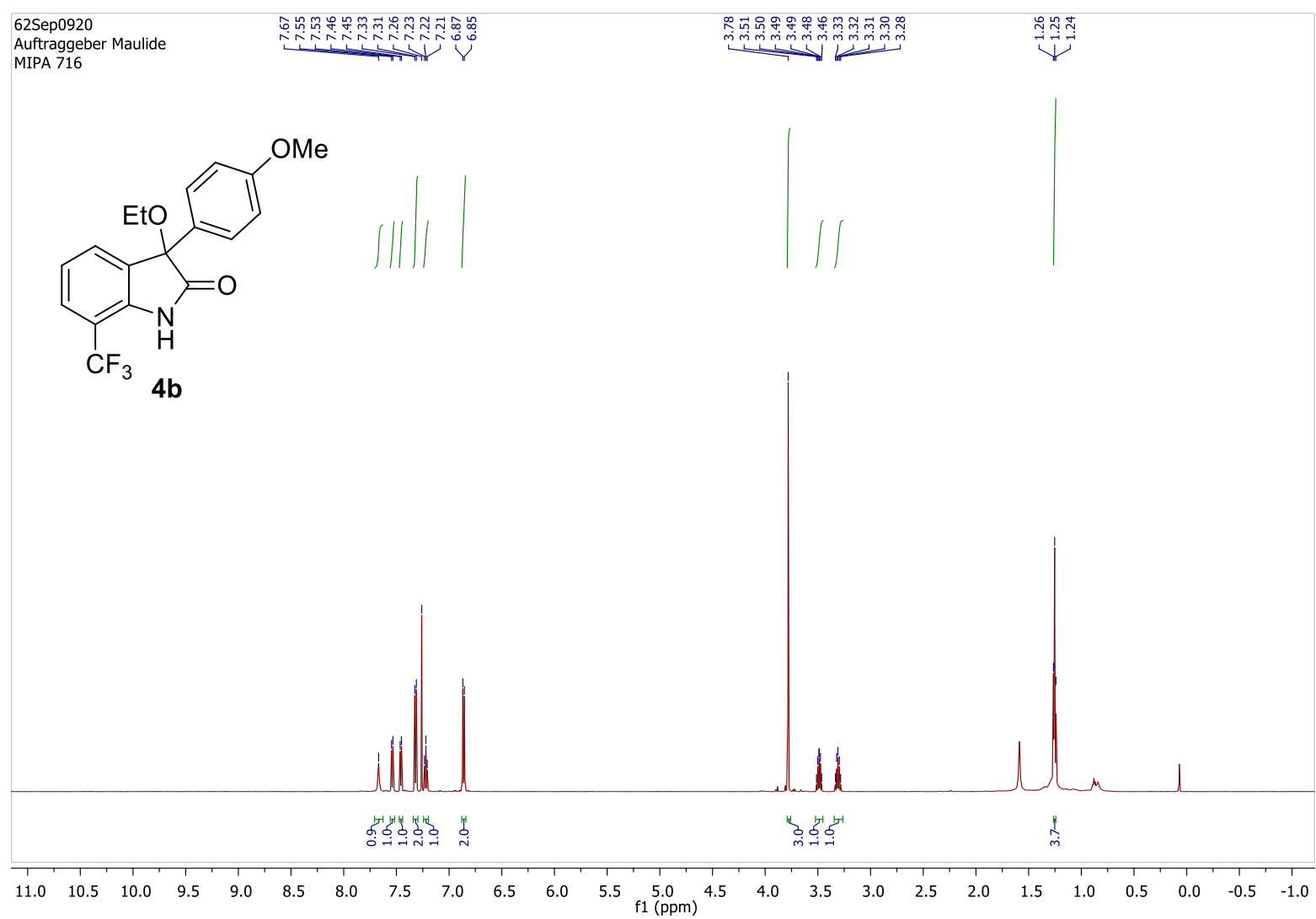

${ }^{1} \mathrm{H} \mathrm{NMR} \mathrm{in} \mathrm{CDCl}_{3}$ at $600 \mathrm{MHz}$

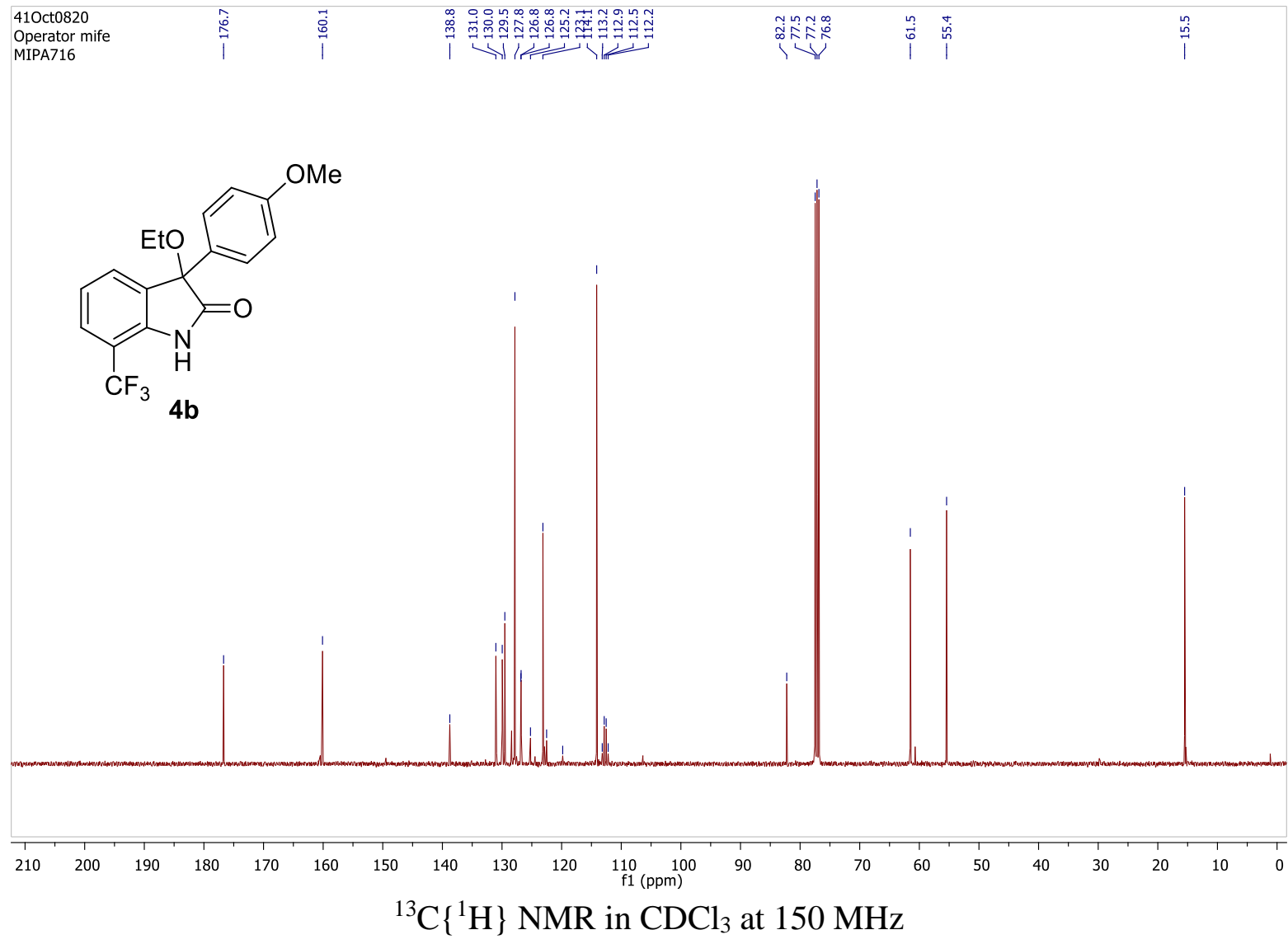


62Sep0920

Auftraggeber Maulide

MIPA 716<smiles>CCC1(c2ccc(OC)cc2)C(=O)Nc2c(C(F)(F)F)cccc21</smiles>

4b

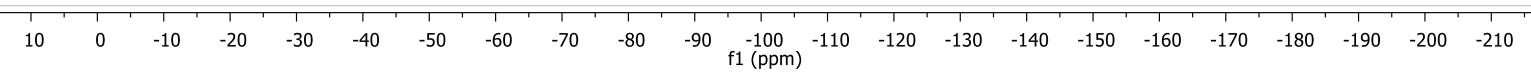

${ }^{19} \mathrm{~F} \mathrm{NMR}$ in $\mathrm{CDCl}_{3}$ at $565 \mathrm{MHz}$ 


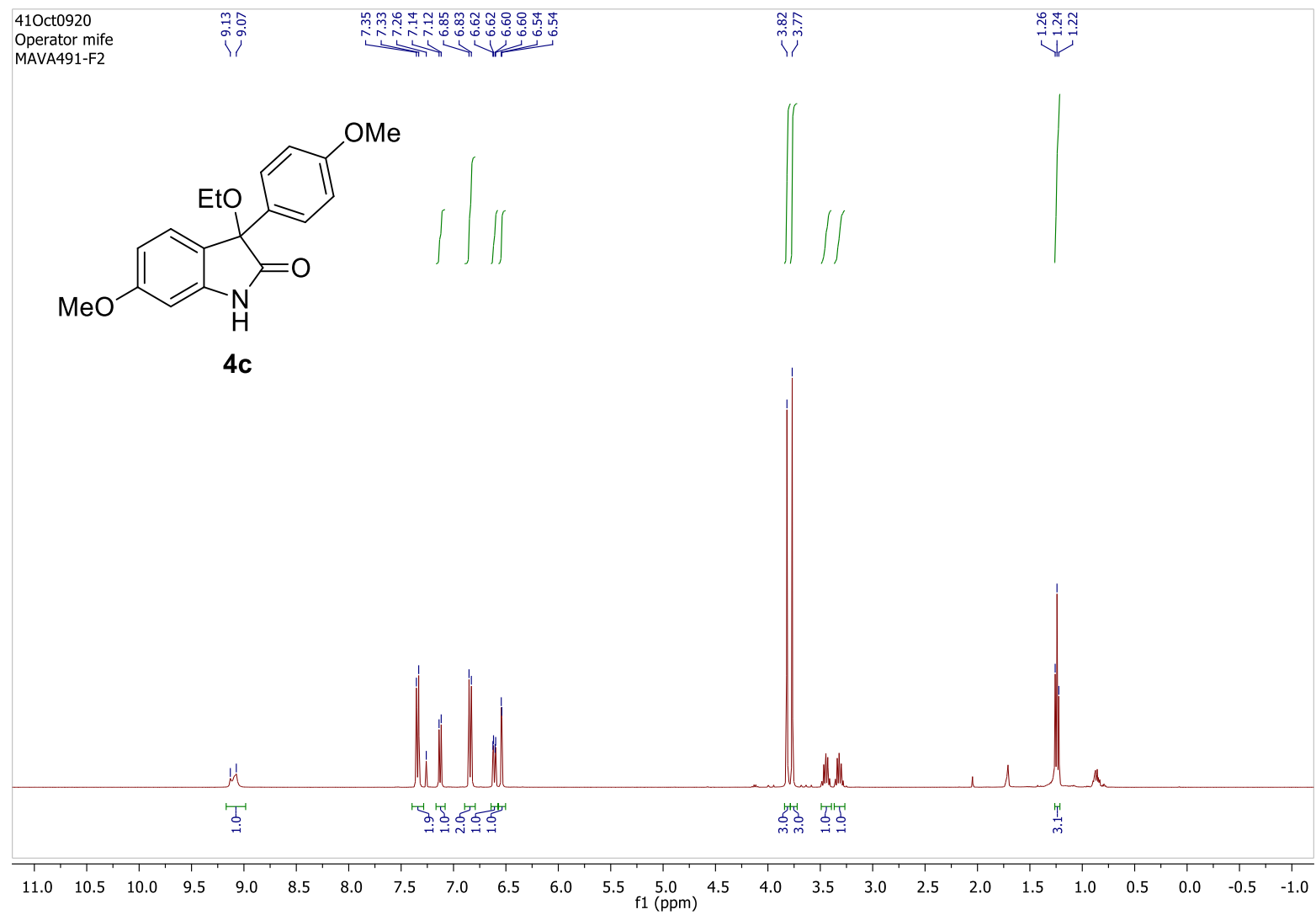

${ }^{1} \mathrm{H} \mathrm{NMR}$ in $\mathrm{CDCl}_{3}$ at $400 \mathrm{MHz}$

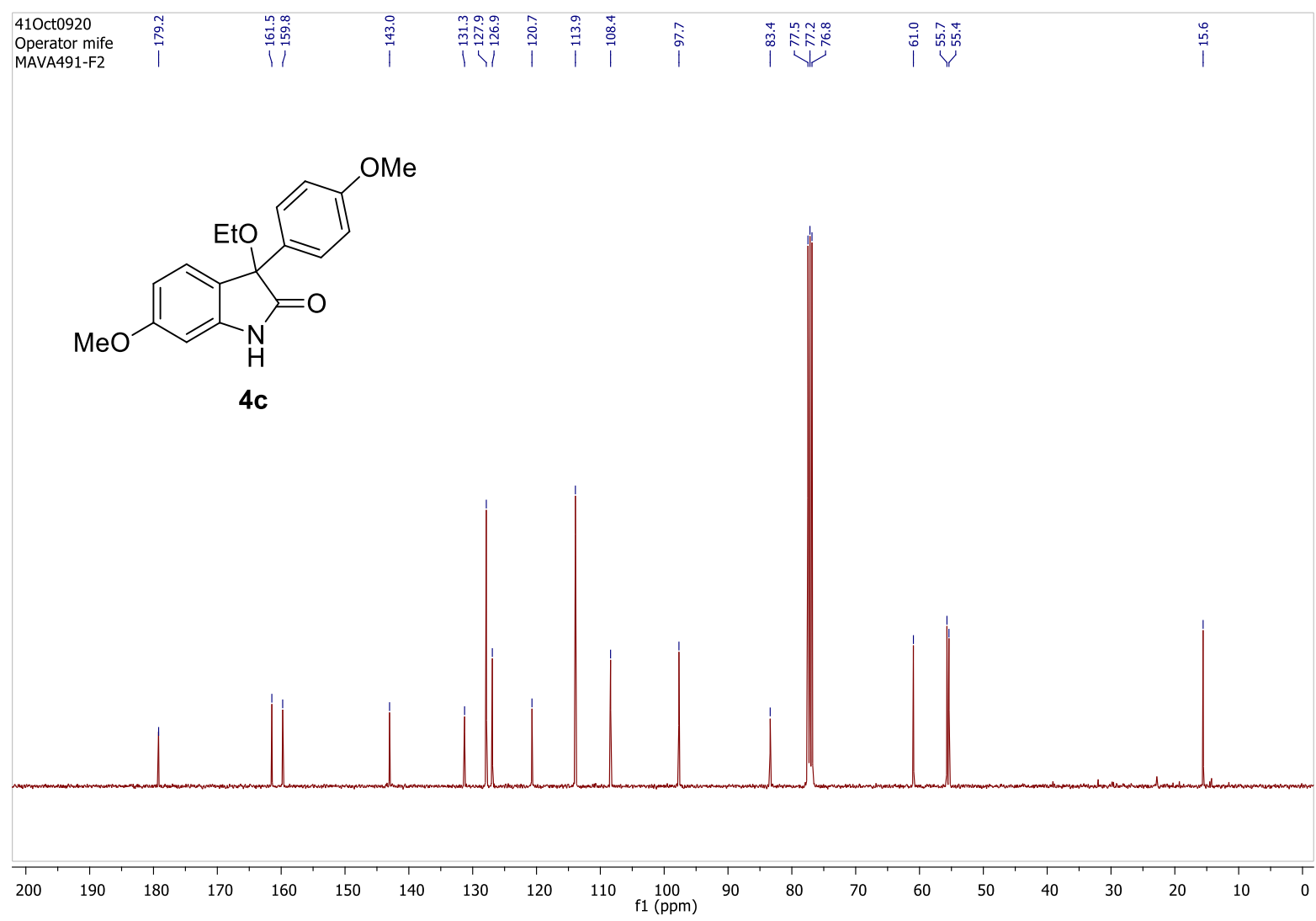

${ }^{13} \mathrm{C}\left\{{ }^{1} \mathrm{H}\right\} \mathrm{NMR}$ in $\mathrm{CDCl}_{3}$ at $100 \mathrm{MHz}$ 


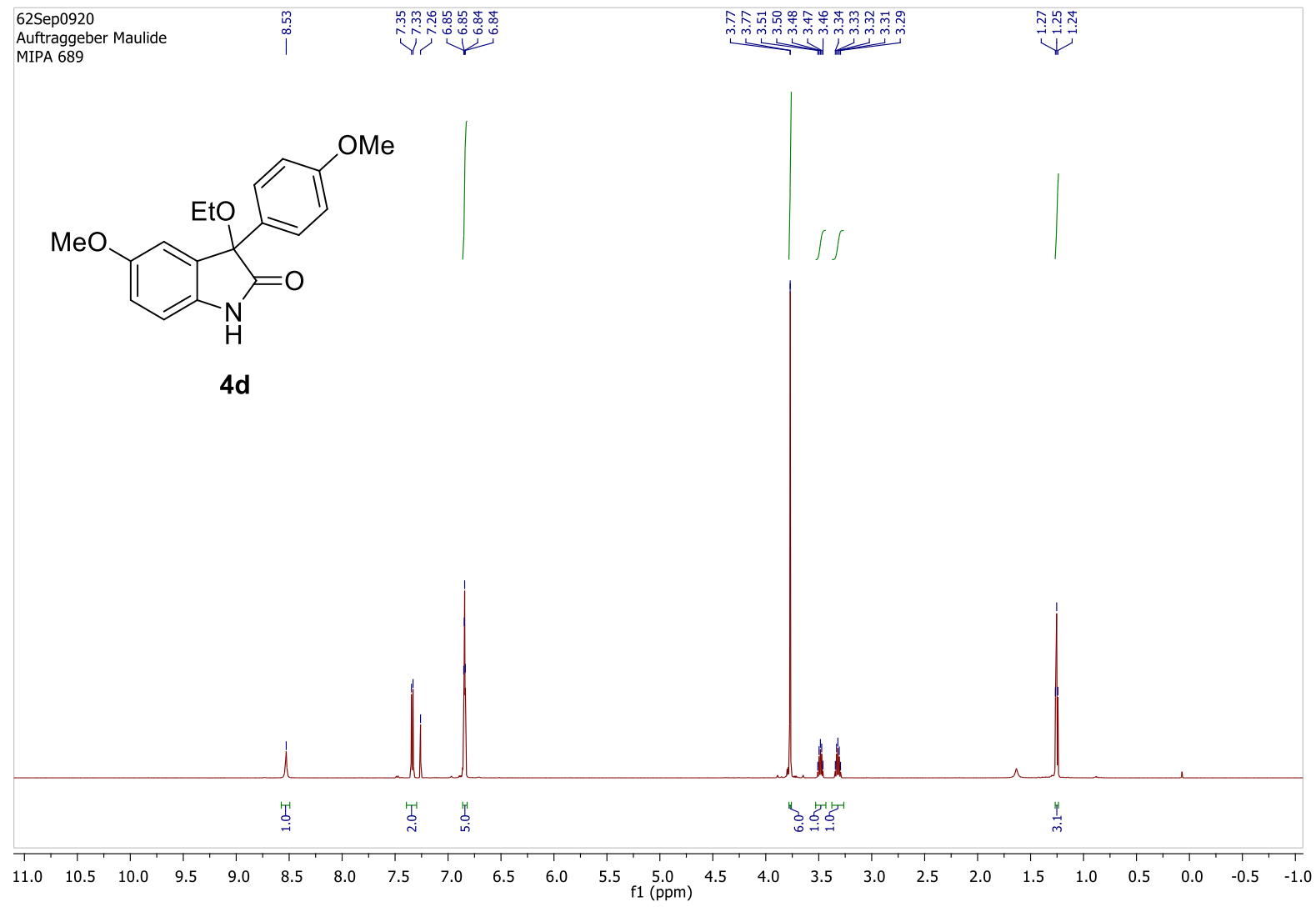

${ }^{1} \mathrm{H} \mathrm{NMR} \mathrm{in} \mathrm{CDCl}_{3}$ at $600 \mathrm{MHz}$

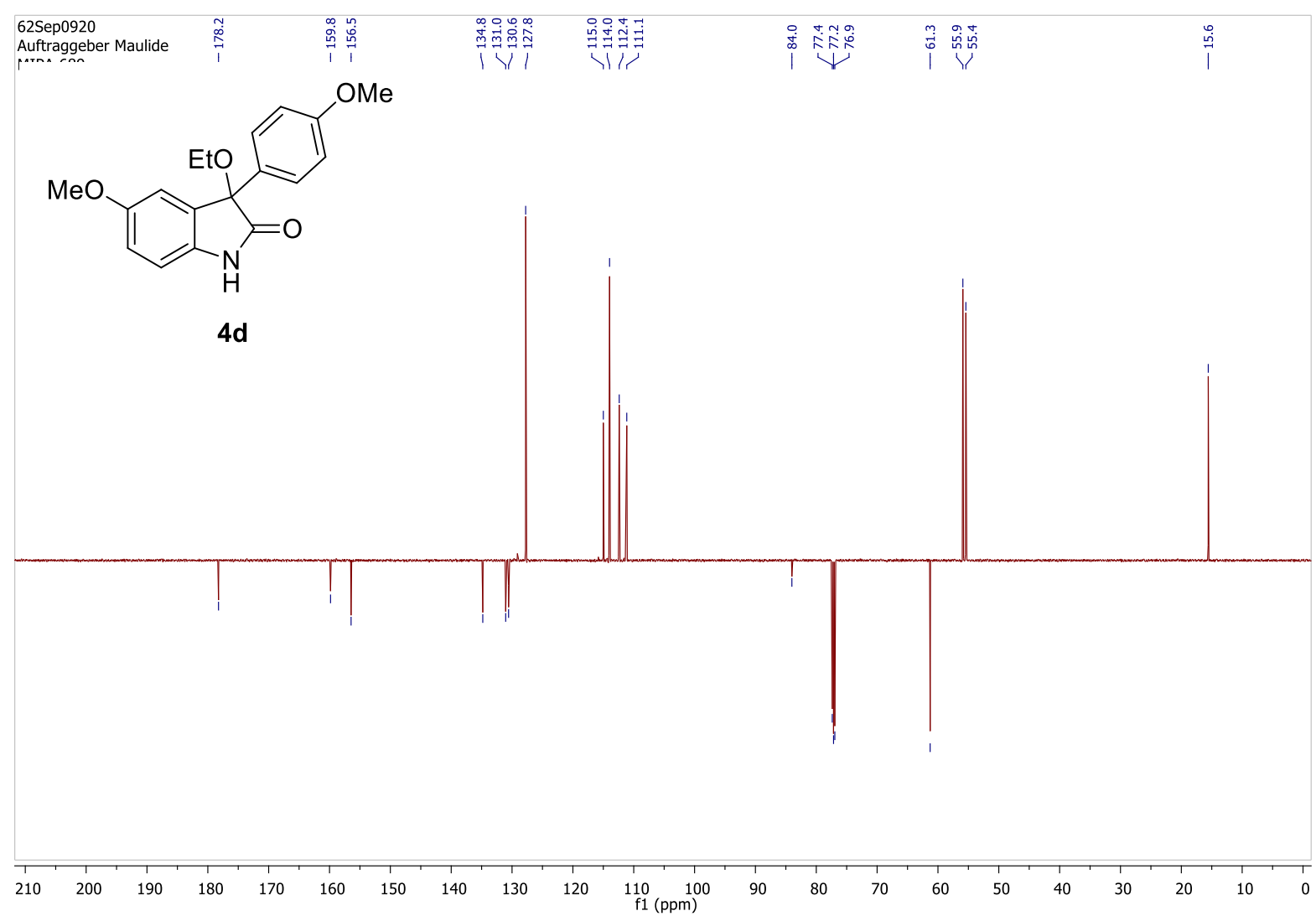

${ }^{13} \mathrm{C}\left\{{ }^{1} \mathrm{H}\right\} \mathrm{NMR}$ in $\mathrm{CDCl}_{3}$ at $150 \mathrm{MHz}$ 


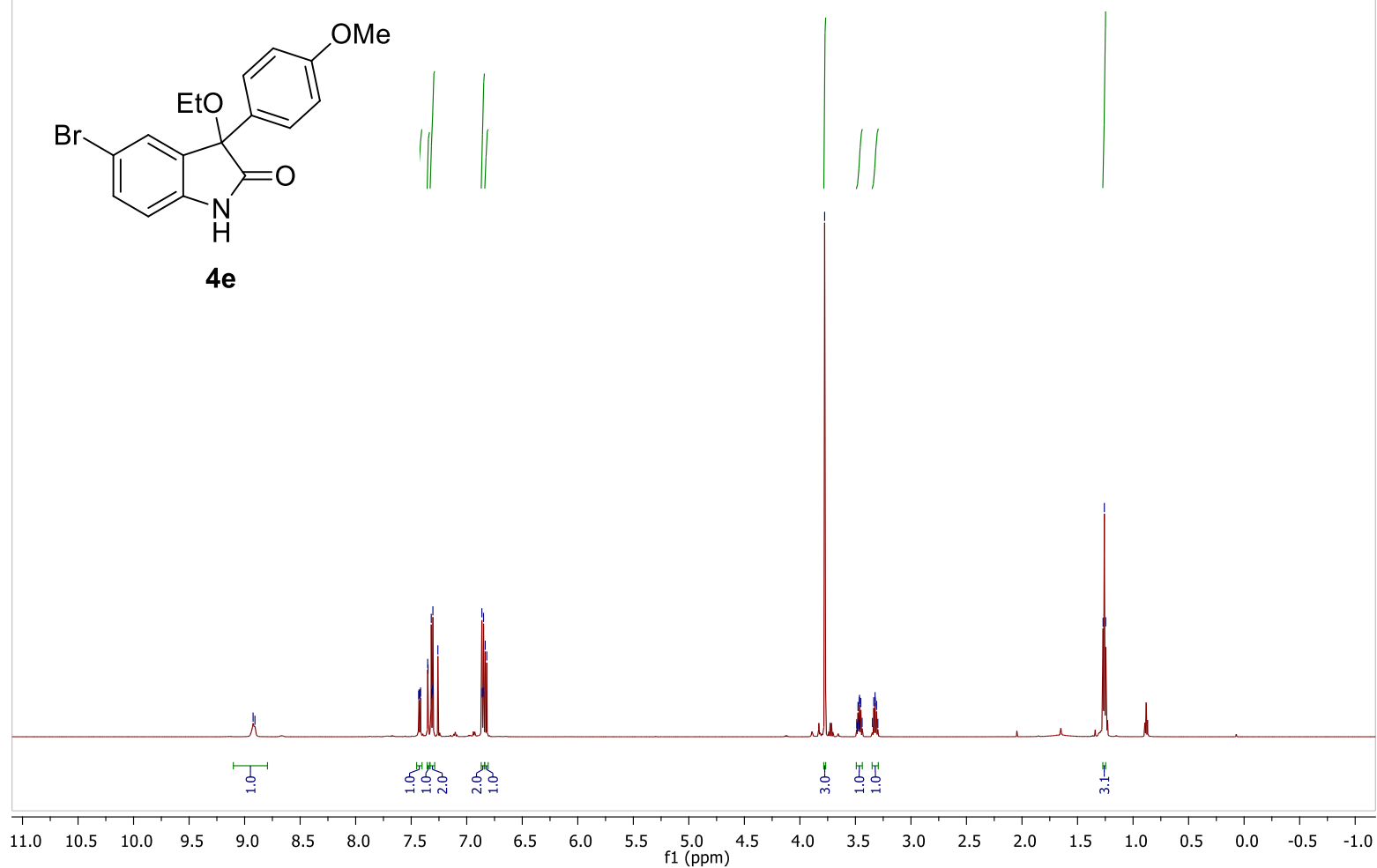

${ }^{1} \mathrm{H} \mathrm{NMR} \mathrm{in} \mathrm{CDCl}_{3}$ at $600 \mathrm{MHz}$

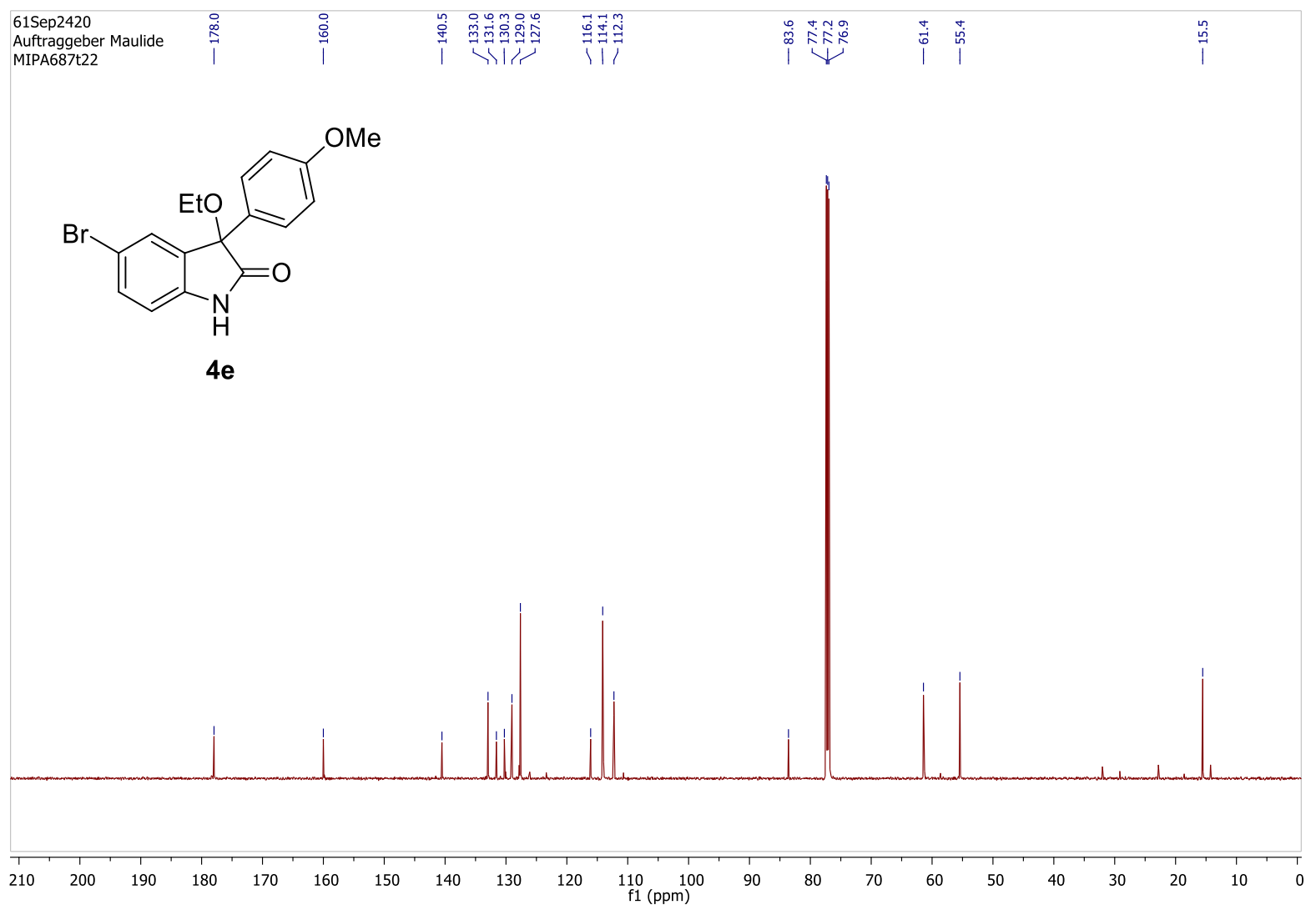

${ }^{13} \mathrm{C}\left\{{ }^{1} \mathrm{H}\right\} \mathrm{NMR}$ in $\mathrm{CDCl}_{3}$ at $150 \mathrm{MHz}$ 


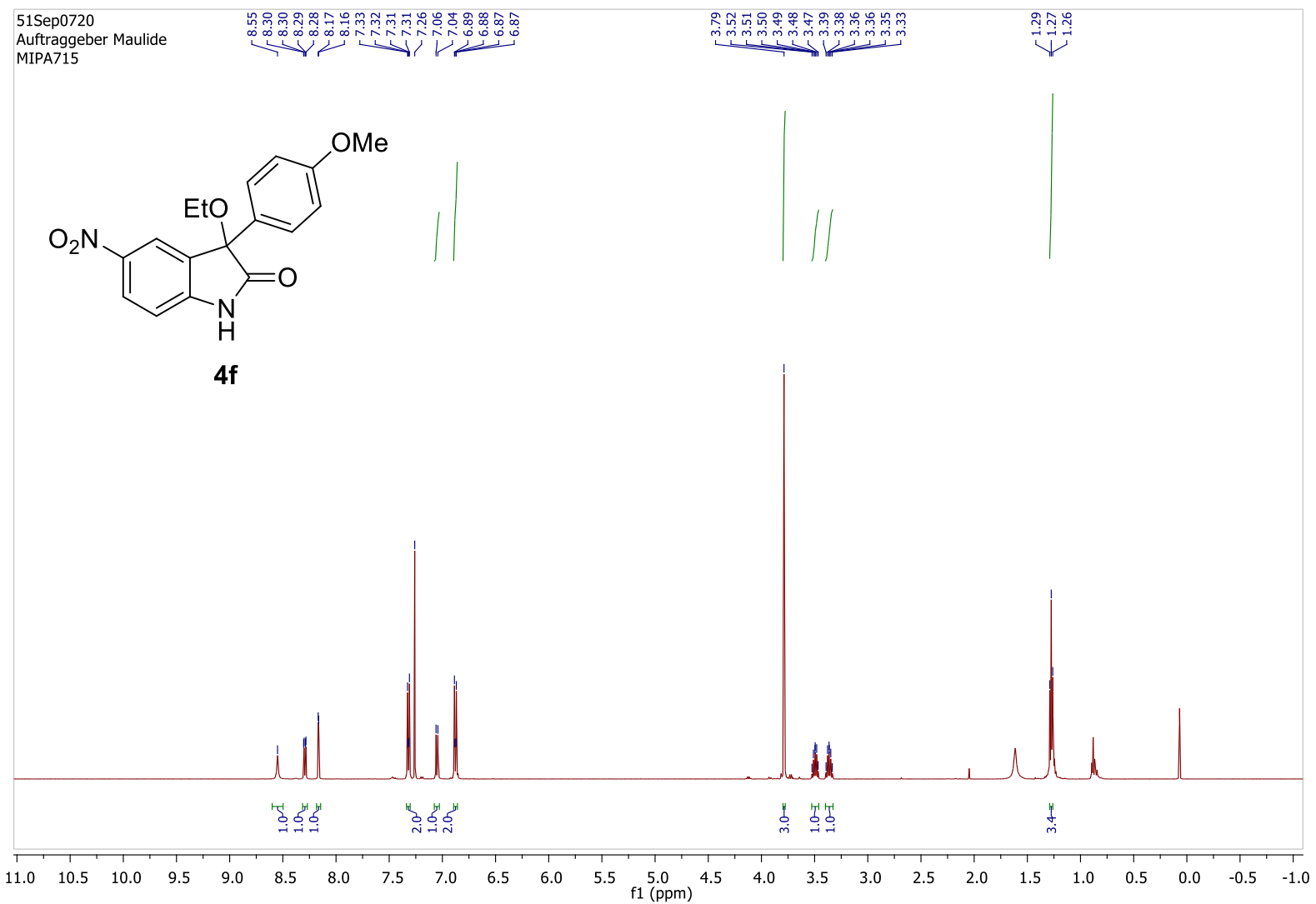

${ }^{1} \mathrm{H} \mathrm{NMR} \mathrm{in} \mathrm{CDCl}_{3}$ at $500 \mathrm{MHz}$

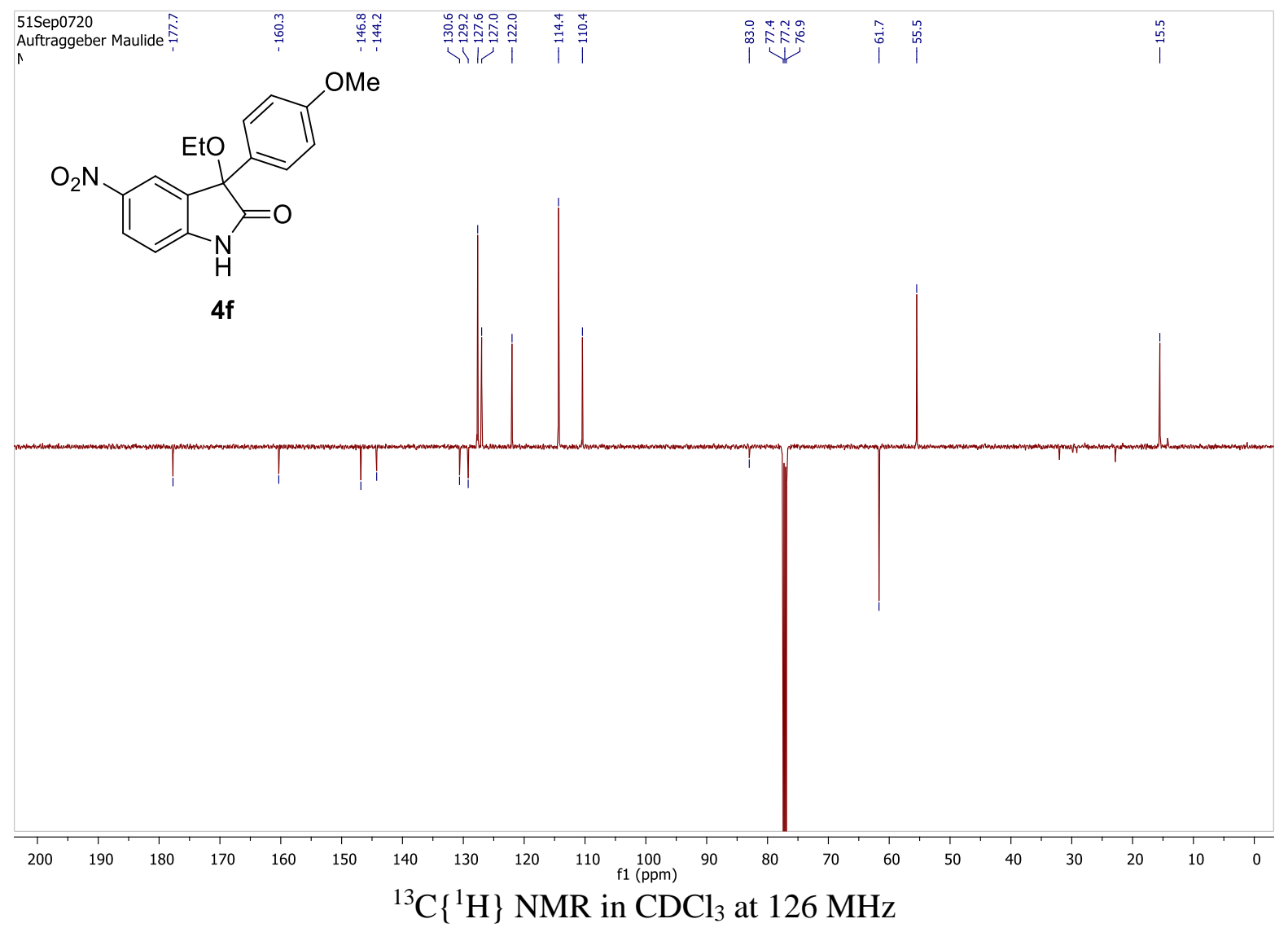




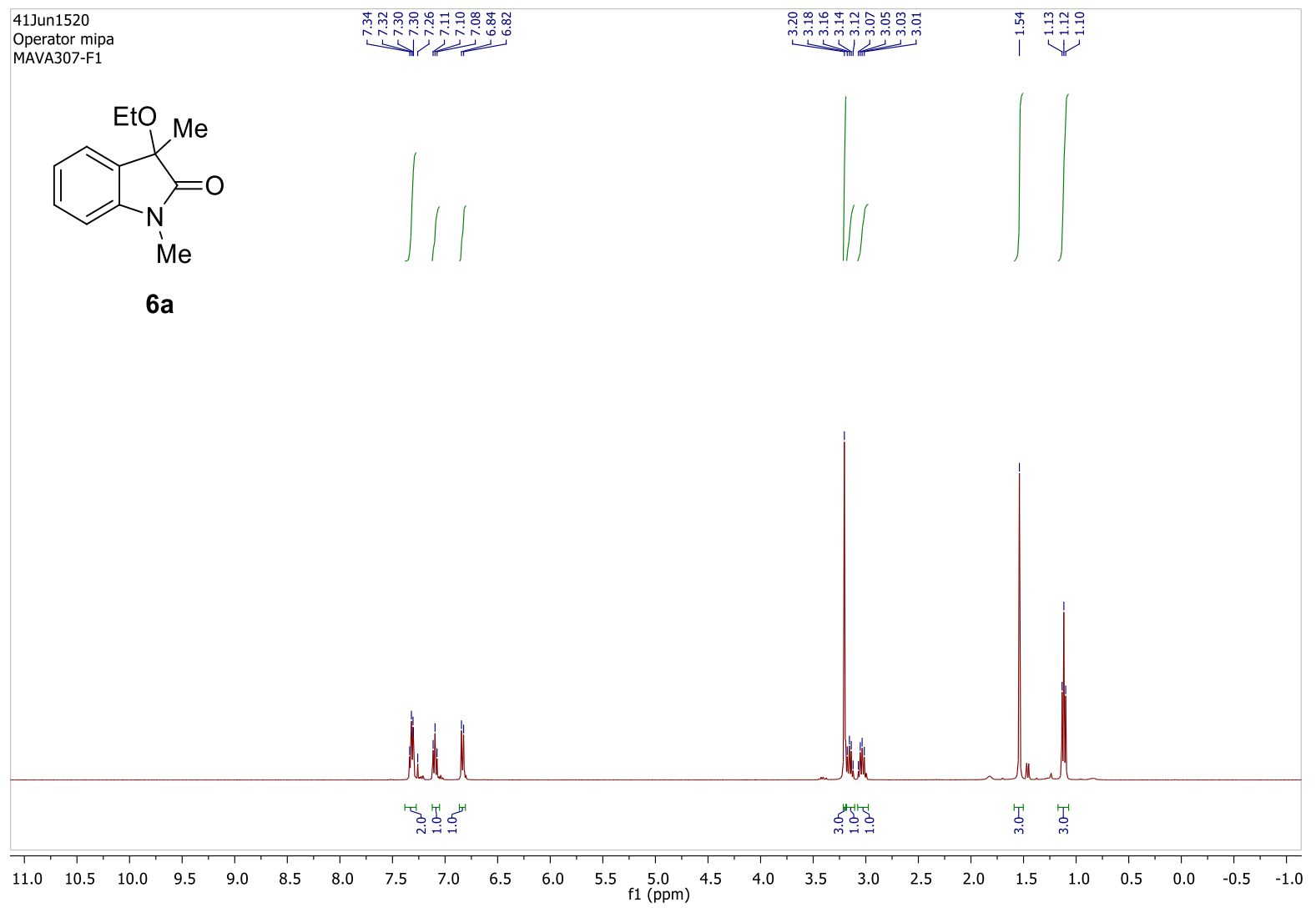

${ }^{1} \mathrm{H} \mathrm{NMR}$ in $\mathrm{CDCl}_{3}$ at $400 \mathrm{MHz}$

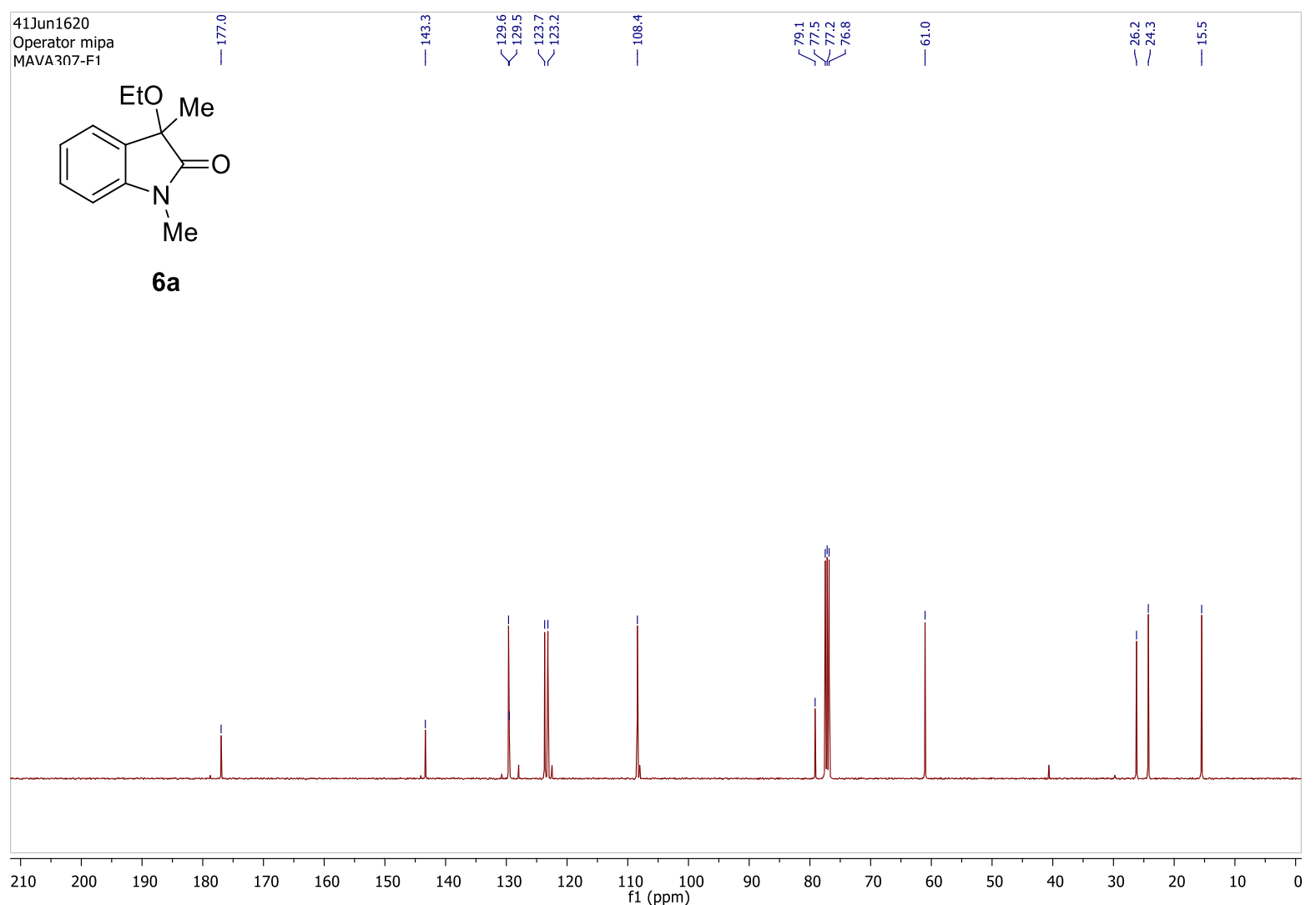

${ }^{13} \mathrm{C}\left\{{ }^{1} \mathrm{H}\right\} \mathrm{NMR}$ in $\mathrm{CDCl}_{3}$ at $100 \mathrm{MHz}$ 


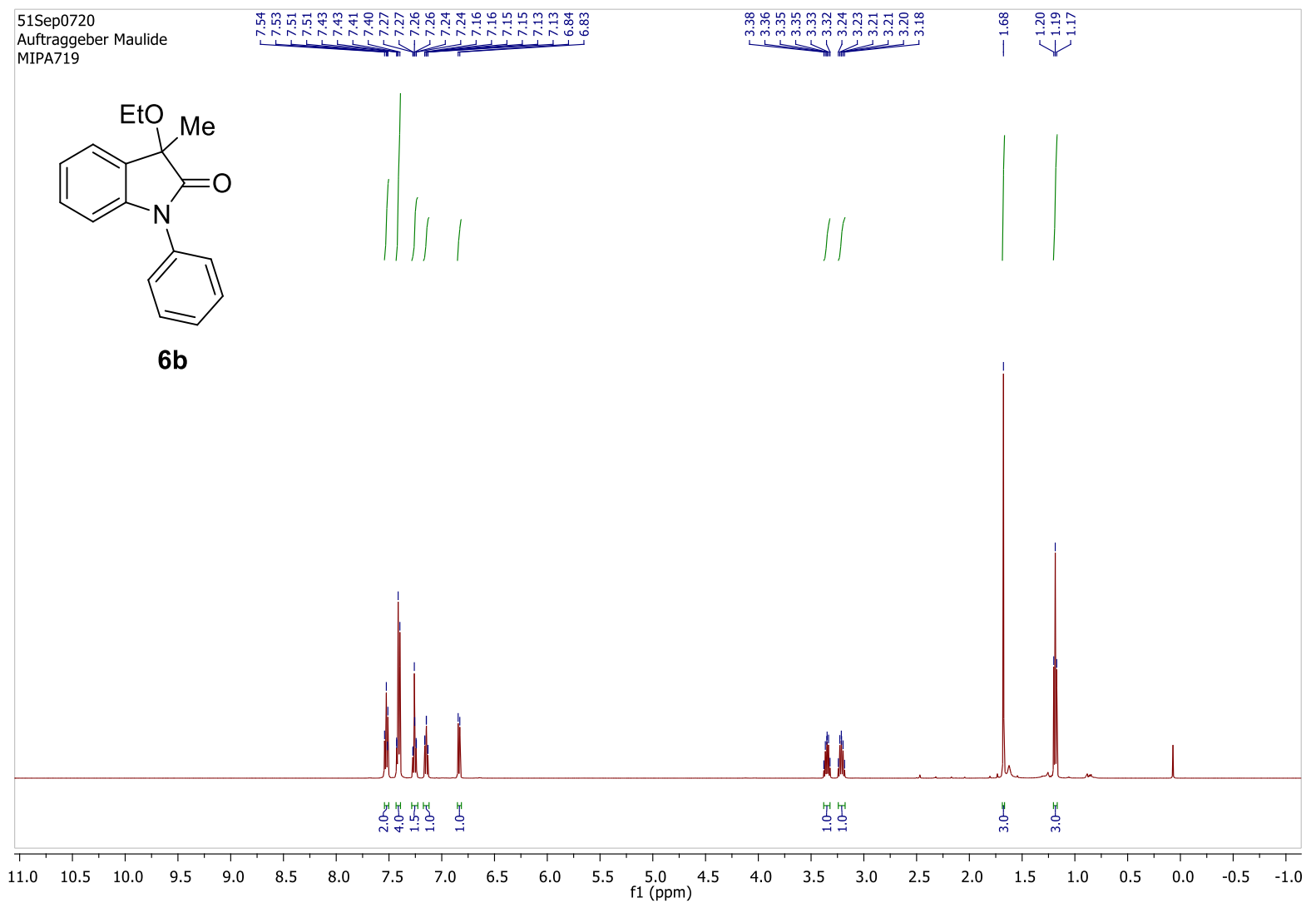

${ }^{1} \mathrm{H} \mathrm{NMR}$ in $\mathrm{CDCl}_{3}$ at $500 \mathrm{MHz}$

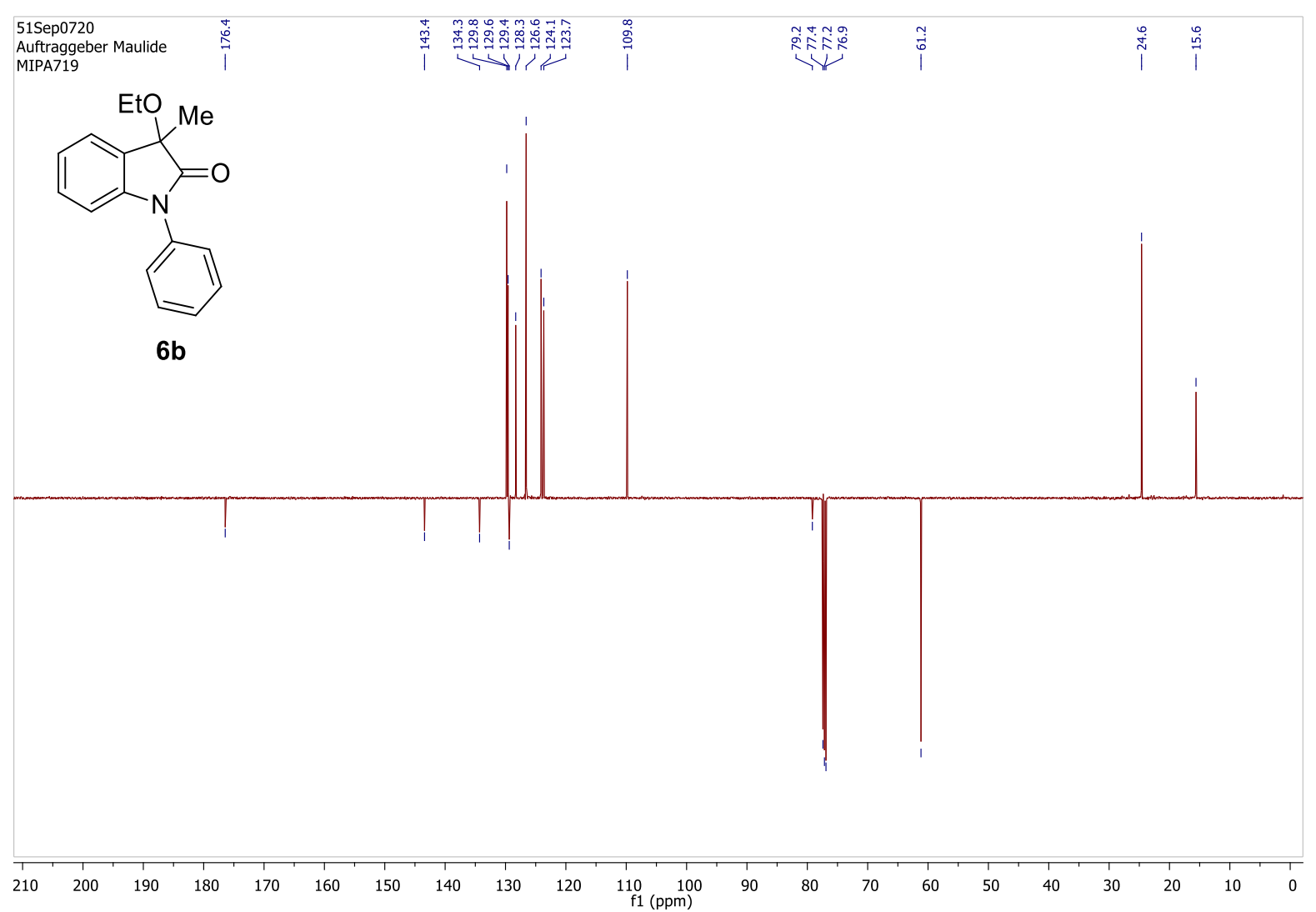

${ }^{13} \mathrm{C}\left\{{ }^{1} \mathrm{H}\right\} \mathrm{NMR}$ in $\mathrm{CDCl}_{3}$ at $126 \mathrm{MHz}$ 


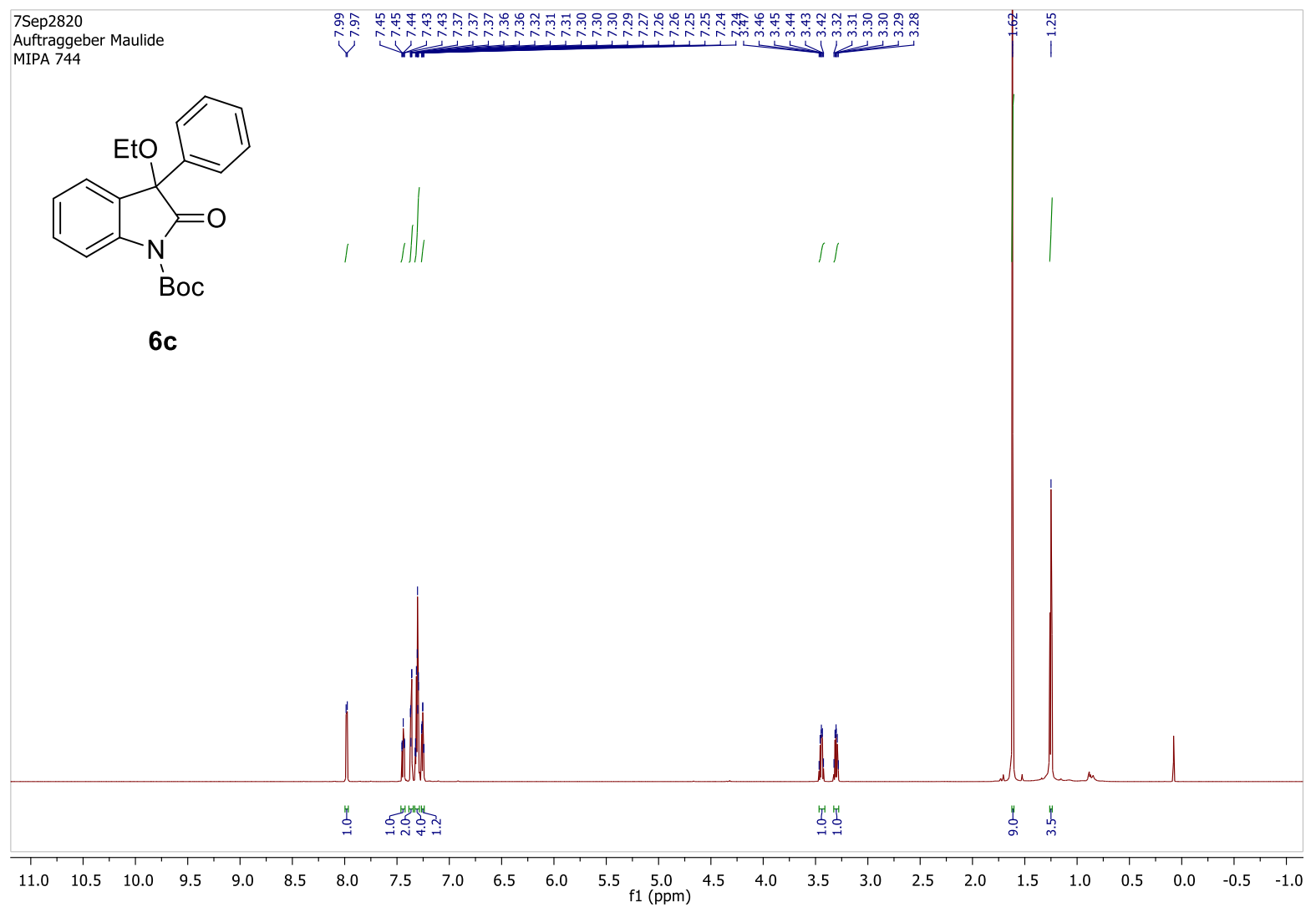

${ }^{1} \mathrm{H} \mathrm{NMR}$ in $\mathrm{CDCl}_{3}$ at $700 \mathrm{MHz}$

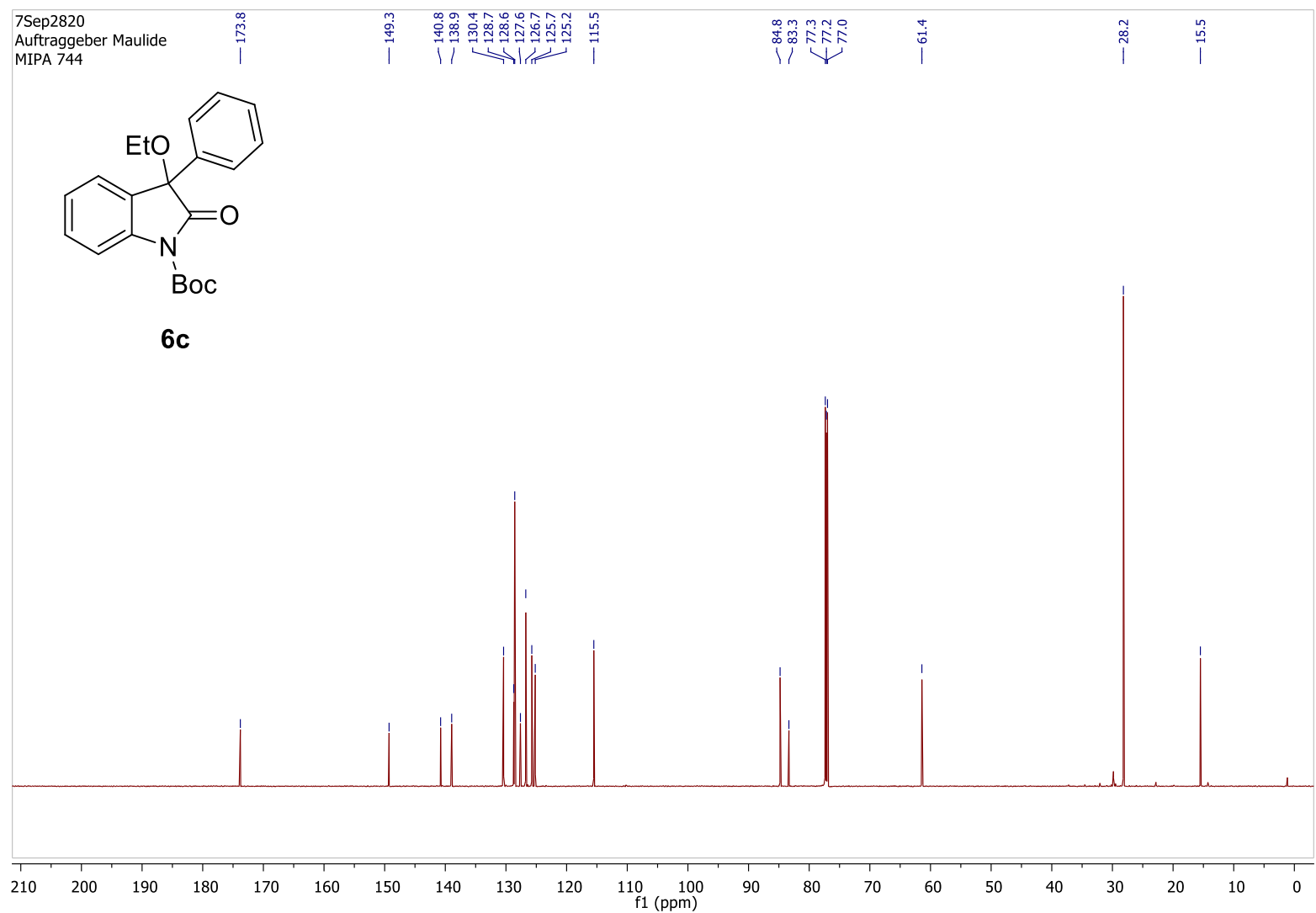

${ }^{13} \mathrm{C}\left\{{ }^{1} \mathrm{H}\right\} \mathrm{NMR}$ in $\mathrm{CDCl}_{3}$ at $176 \mathrm{MHz}$ 


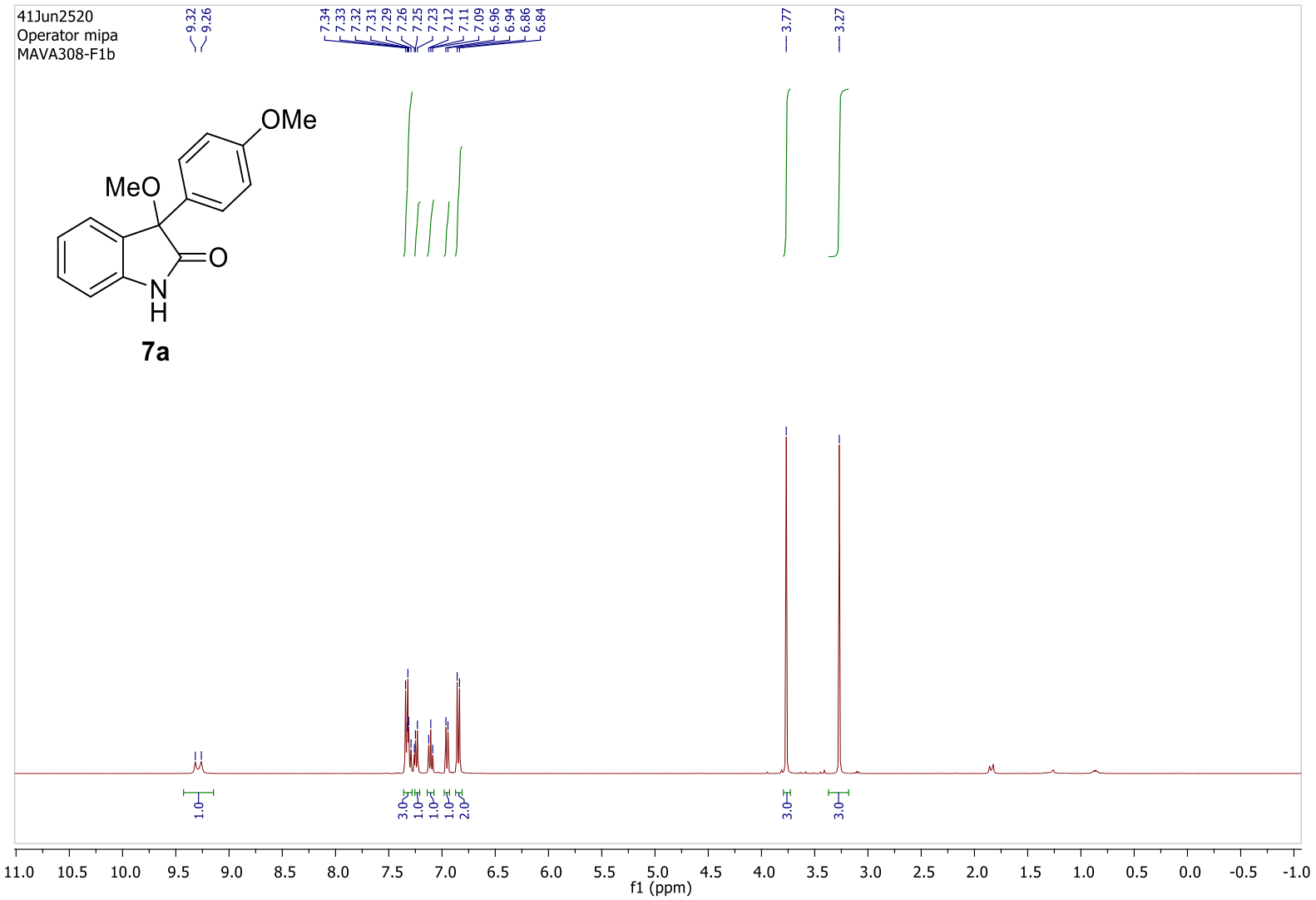

${ }^{1} \mathrm{H} \mathrm{NMR} \mathrm{in} \mathrm{CDCl}_{3}$ at $400 \mathrm{MHz}$

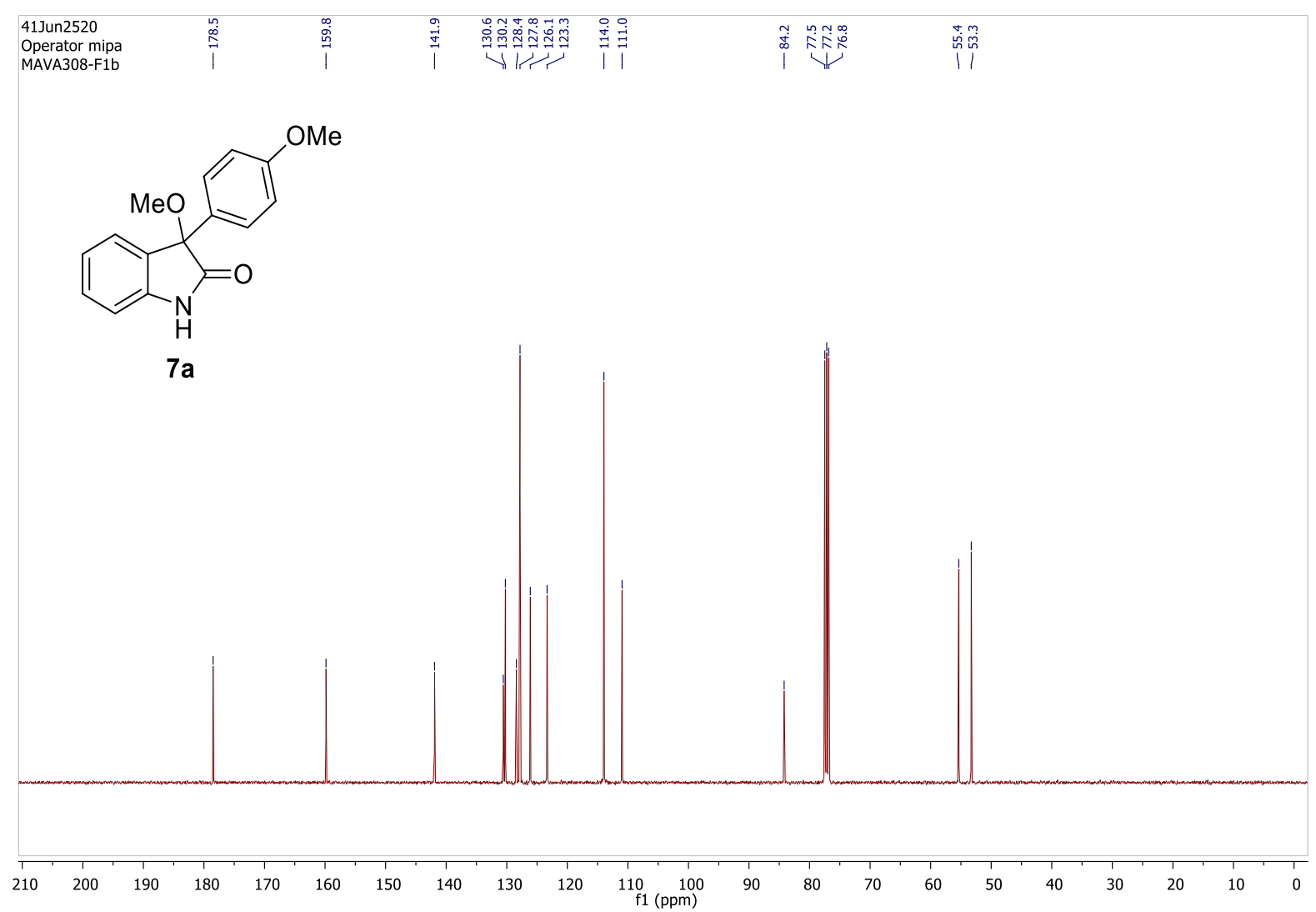

${ }^{13} \mathrm{C}\left\{{ }^{1} \mathrm{H}\right\} \mathrm{NMR}$ in $\mathrm{CDCl}_{3}$ at $100 \mathrm{MHz}$ 
<smiles>C=CCOC1(c2ccc(OC)cc2)C(=O)Nc2ccccc21</smiles>

$7 b$
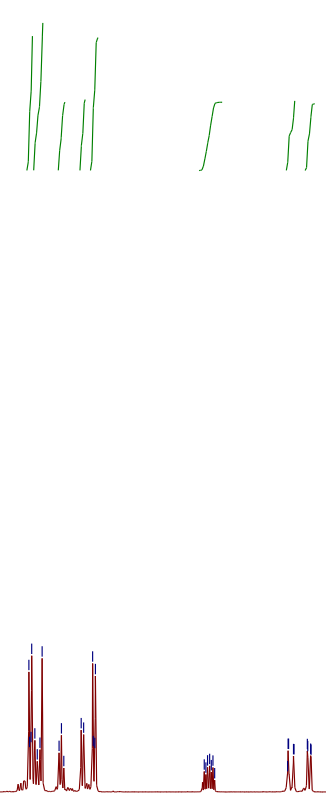

in

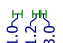

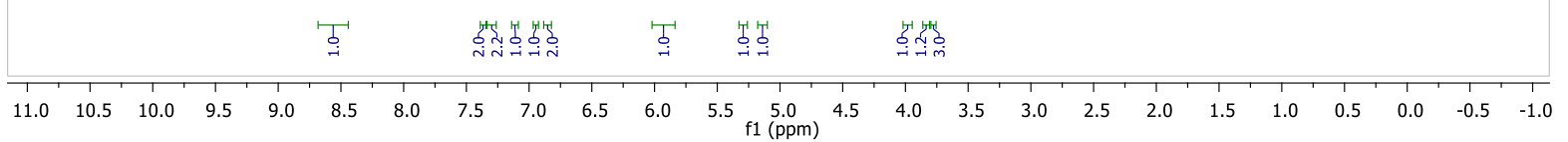

${ }^{1} \mathrm{H} \mathrm{NMR}$ in $\mathrm{CDCl}_{3}$ at $400 \mathrm{MHz}$

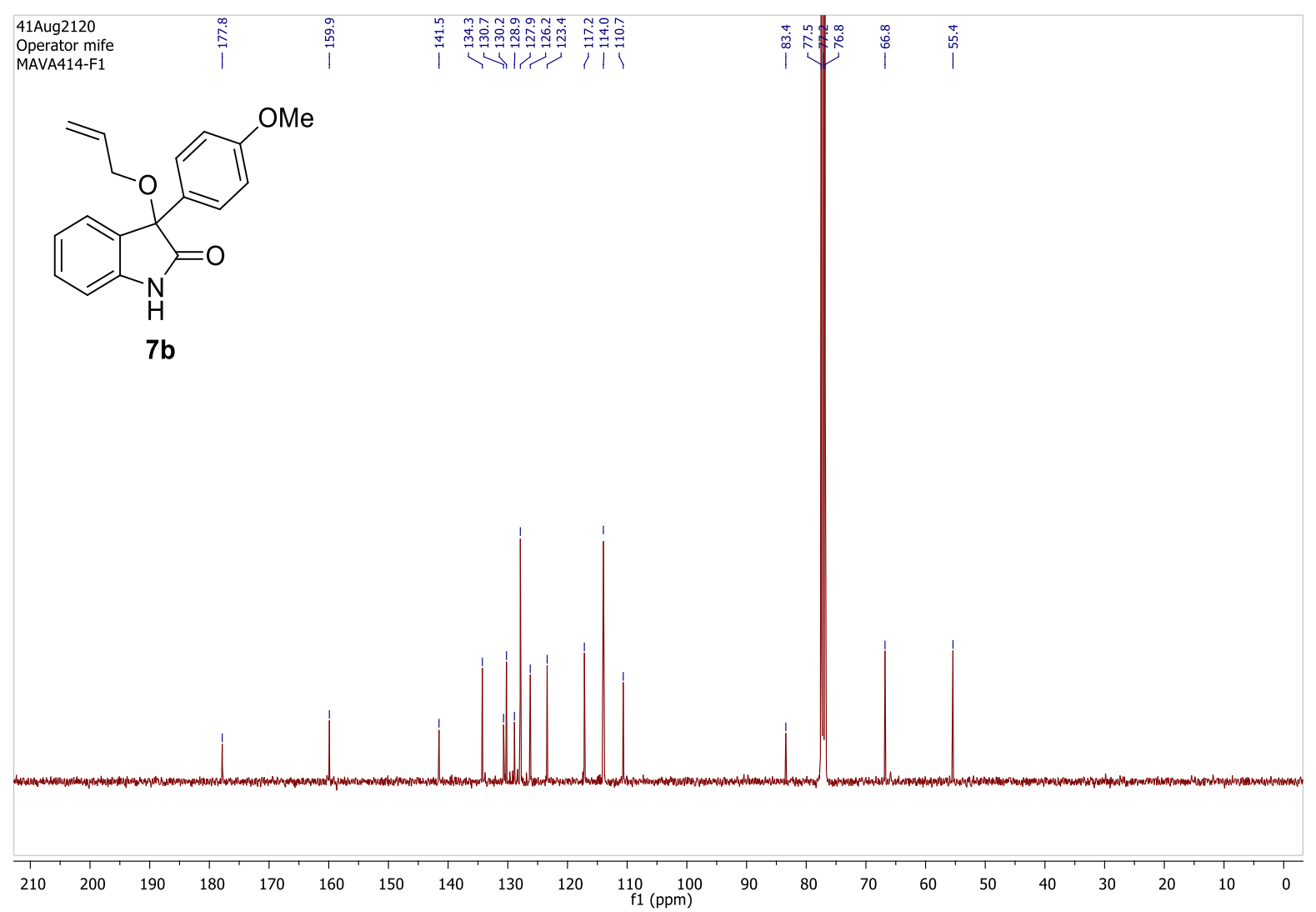

${ }^{13} \mathrm{C}\left\{{ }^{1} \mathrm{H}\right\} \mathrm{NMR}$ in $\mathrm{CDCl}_{3}$ at $100 \mathrm{MHz}$ 


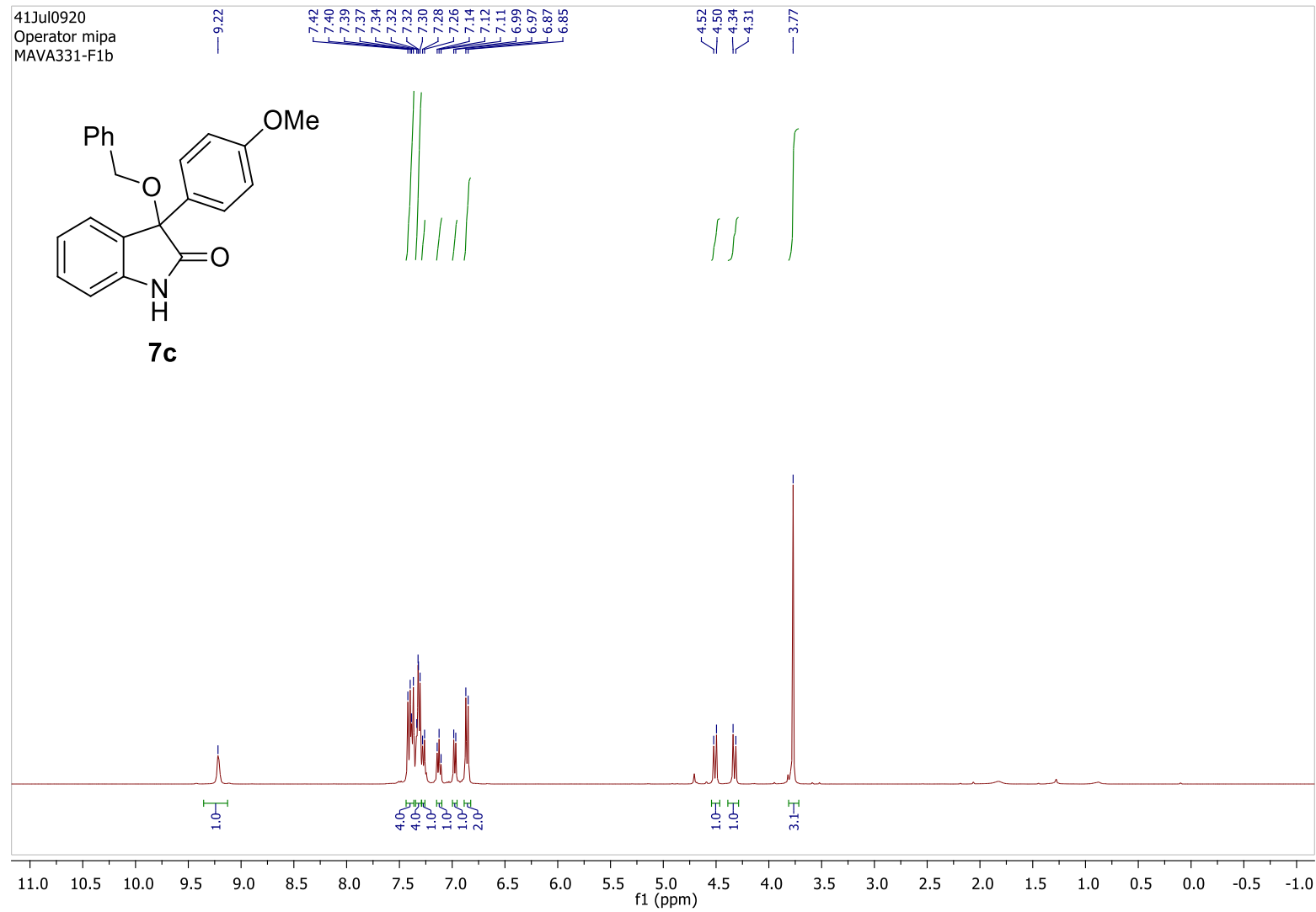

${ }^{1} \mathrm{H} \mathrm{NMR} \mathrm{in} \mathrm{CDCl}_{3}$ at $400 \mathrm{MHz}$

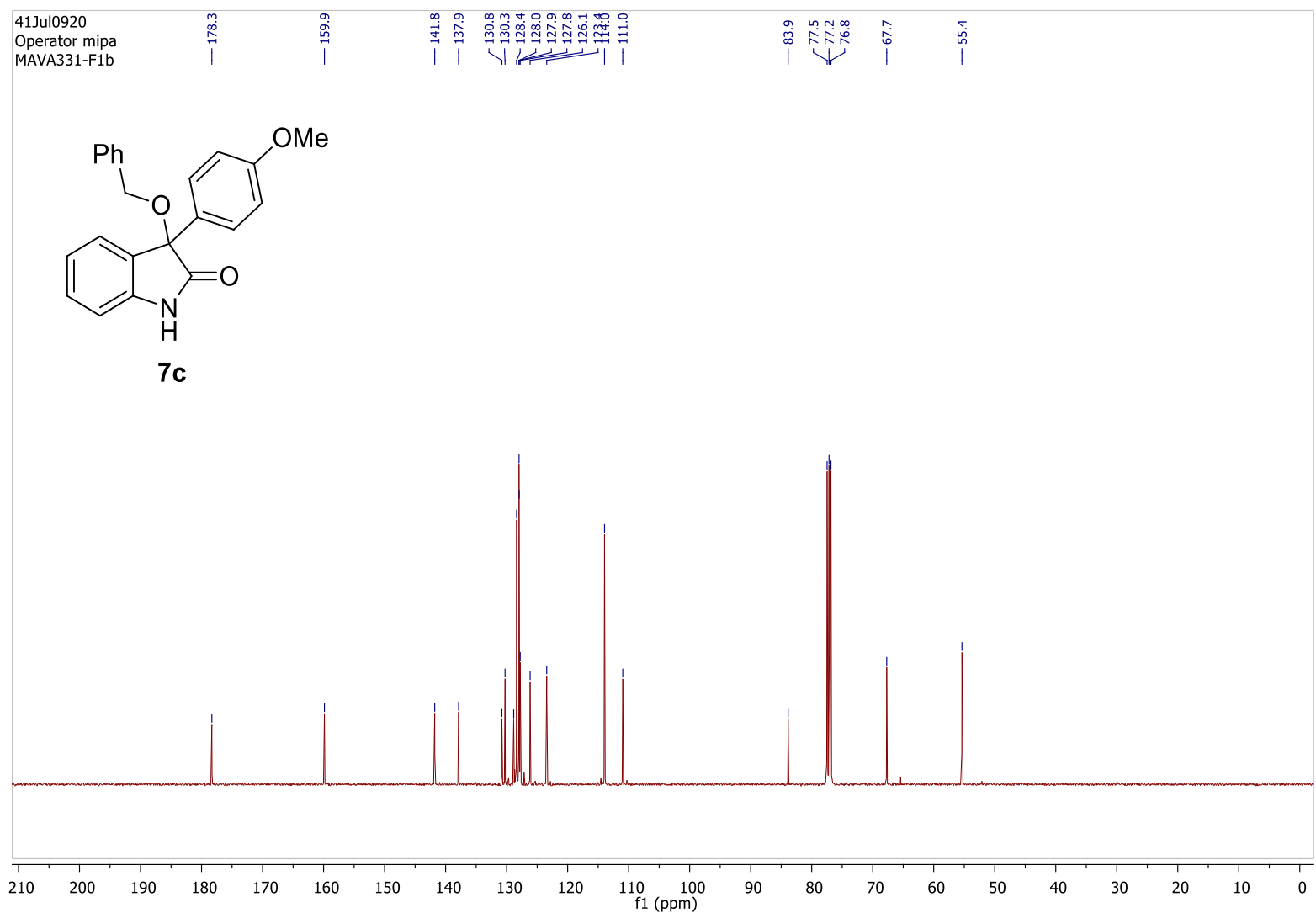

${ }^{13} \mathrm{C}\left\{{ }^{1} \mathrm{H}\right\} \mathrm{NMR}$ in $\mathrm{CDCl}_{3}$ at $100 \mathrm{MHz}$ 
61Aug2620
Auftraggeber Maulide
MIPA 626

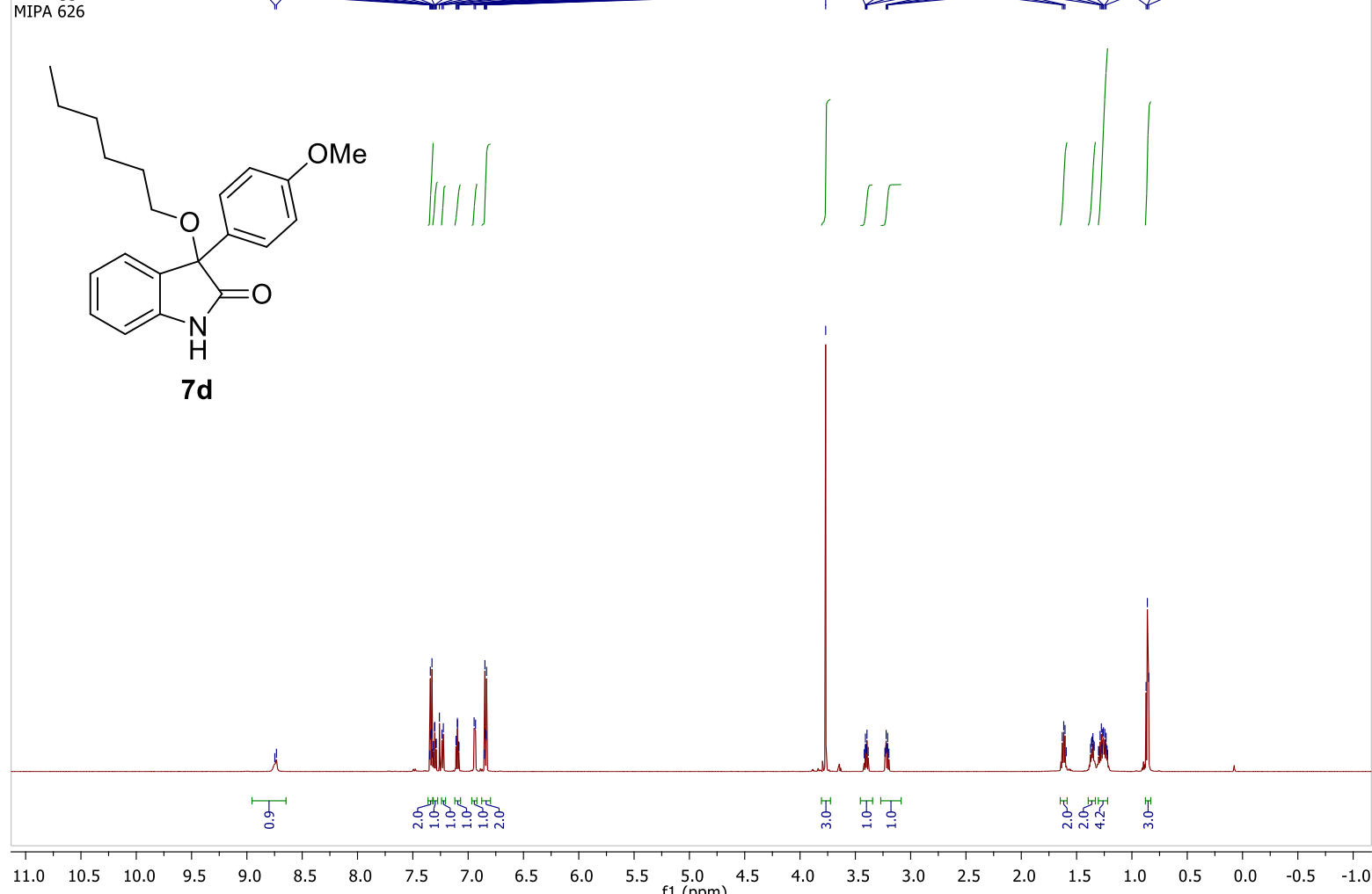

${ }^{1} \mathrm{H} \mathrm{NMR} \mathrm{in} \mathrm{CDCl}_{3}$ at $600 \mathrm{MHz}$

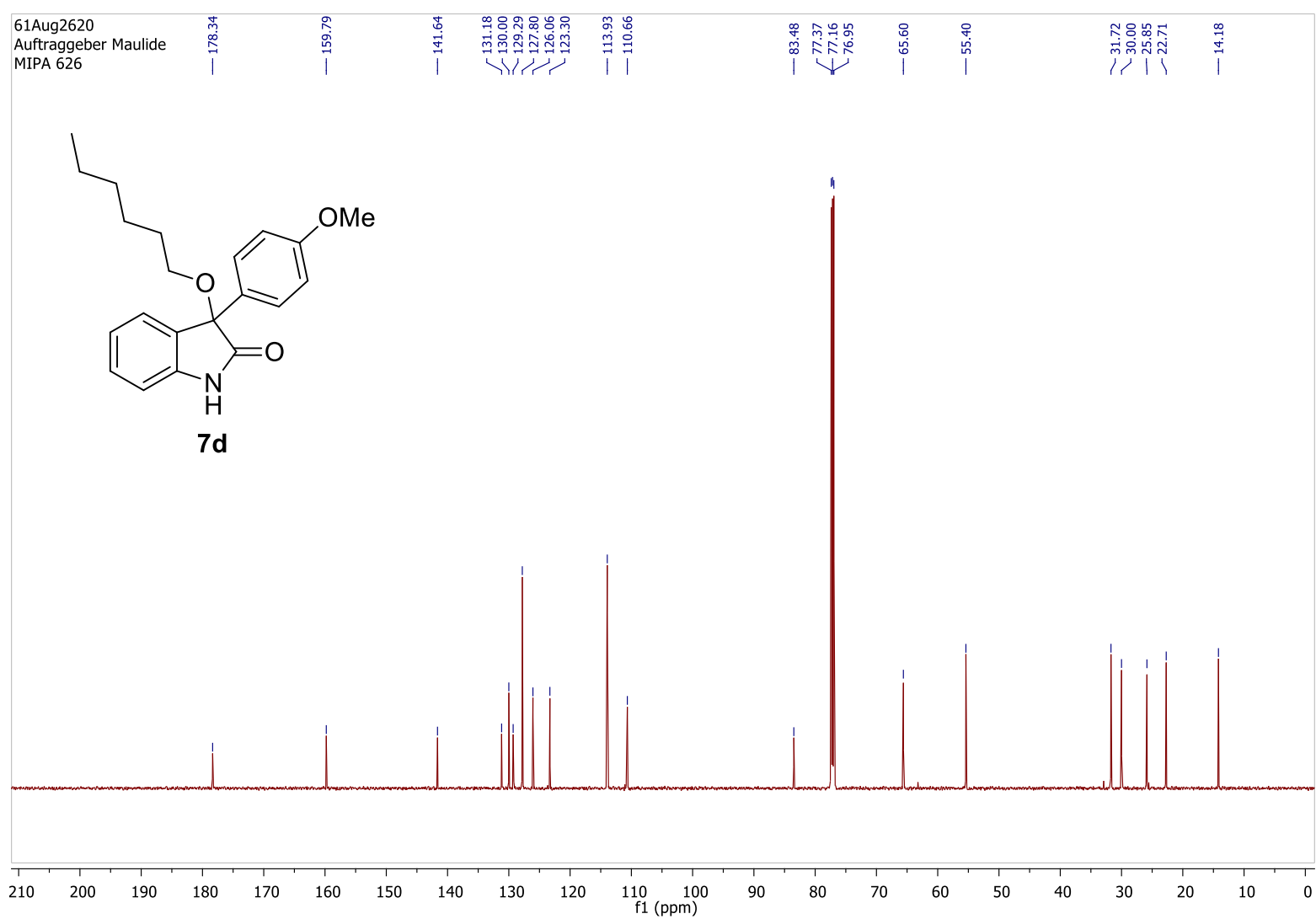

${ }^{13} \mathrm{C}\left\{{ }^{1} \mathrm{H}\right\} \mathrm{NMR}$ in $\mathrm{CDCl}_{3}$ at $150 \mathrm{MHz}$ 


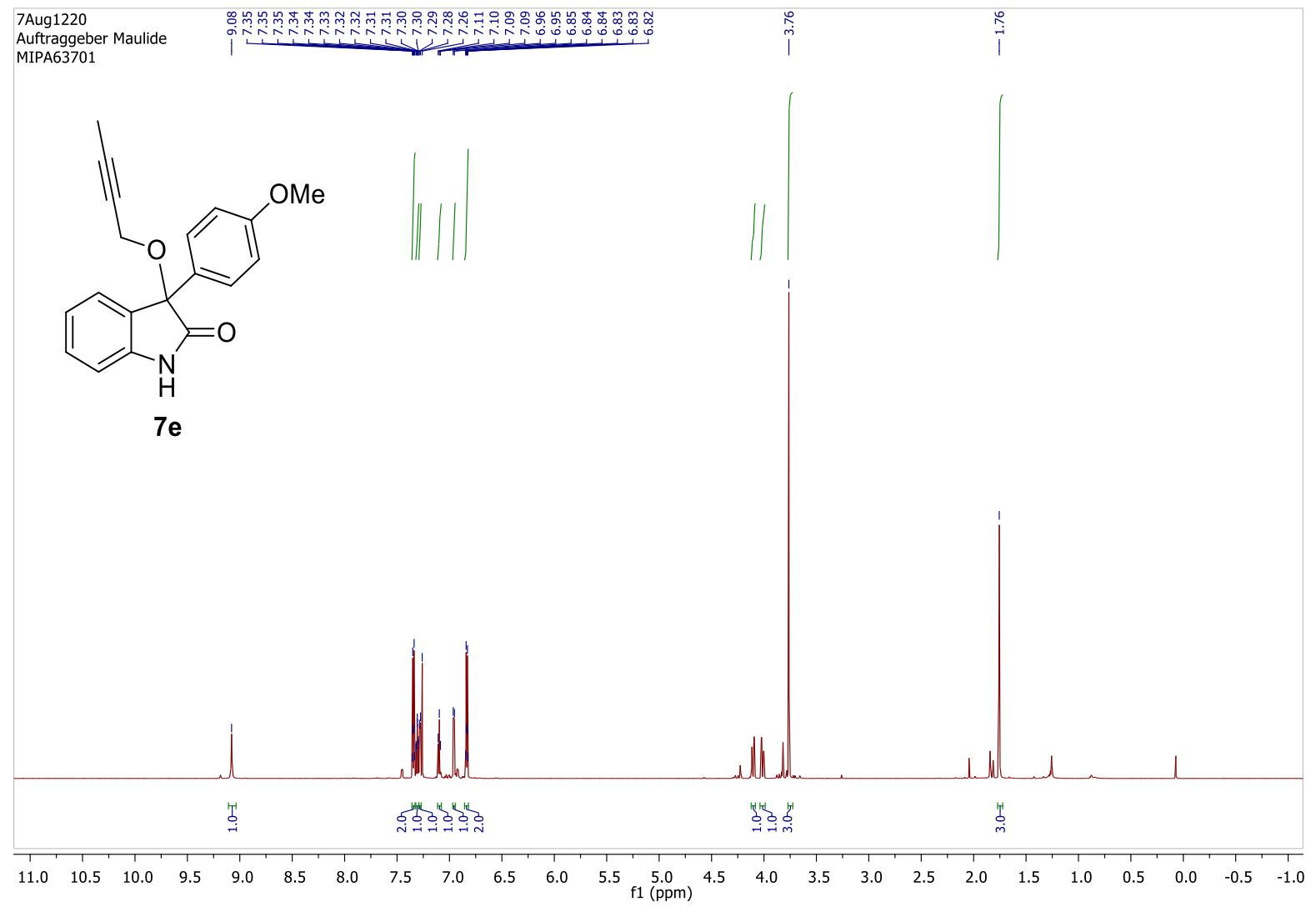

${ }^{1} \mathrm{H} \mathrm{NMR}$ in $\mathrm{CDCl}_{3}$ at $700 \mathrm{MHz}$

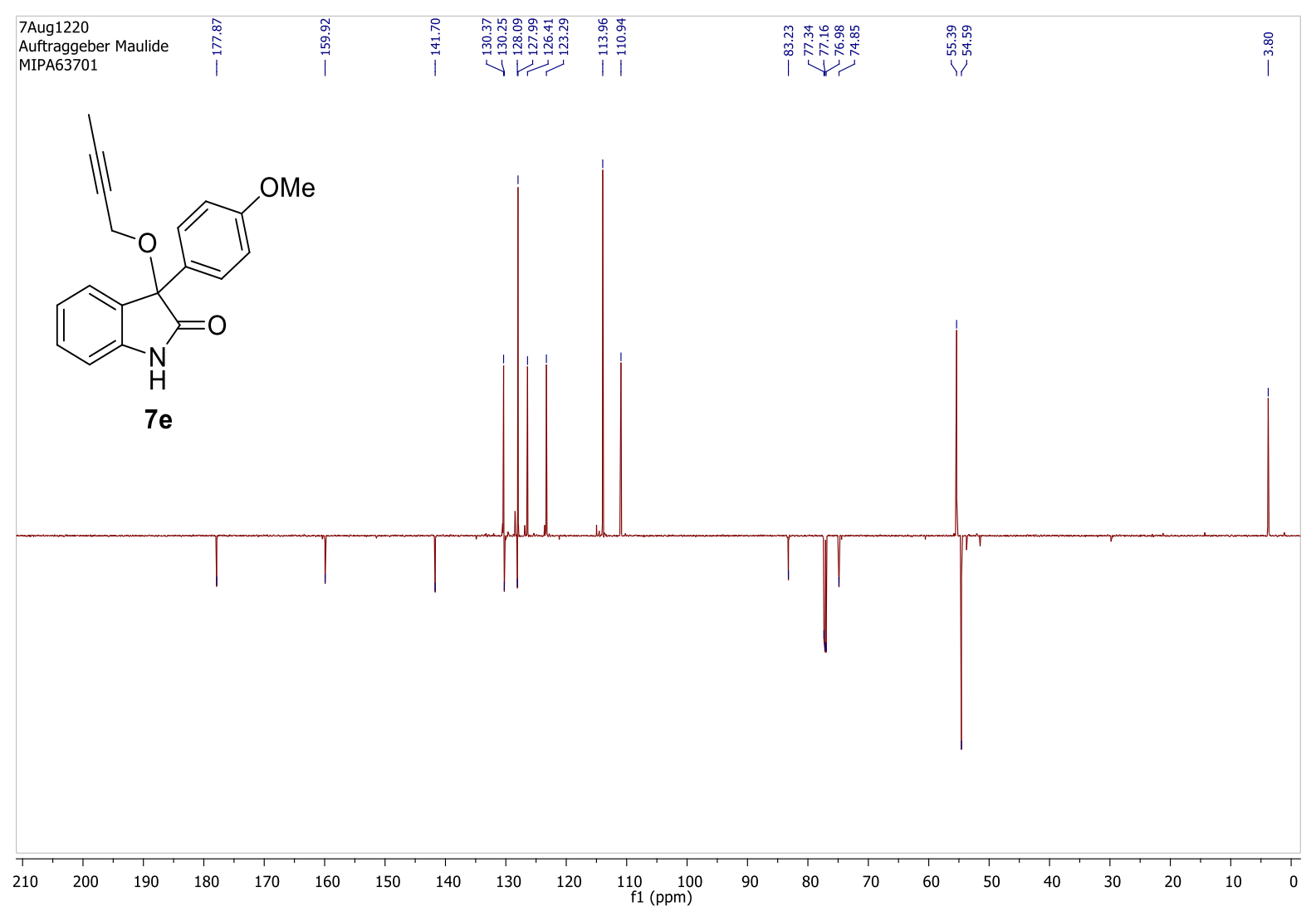

${ }^{13} \mathrm{C}\left\{{ }^{1} \mathrm{H}\right\} \mathrm{NMR}$ in $\mathrm{CDCl}_{3}$ at $176 \mathrm{MHz}$ 


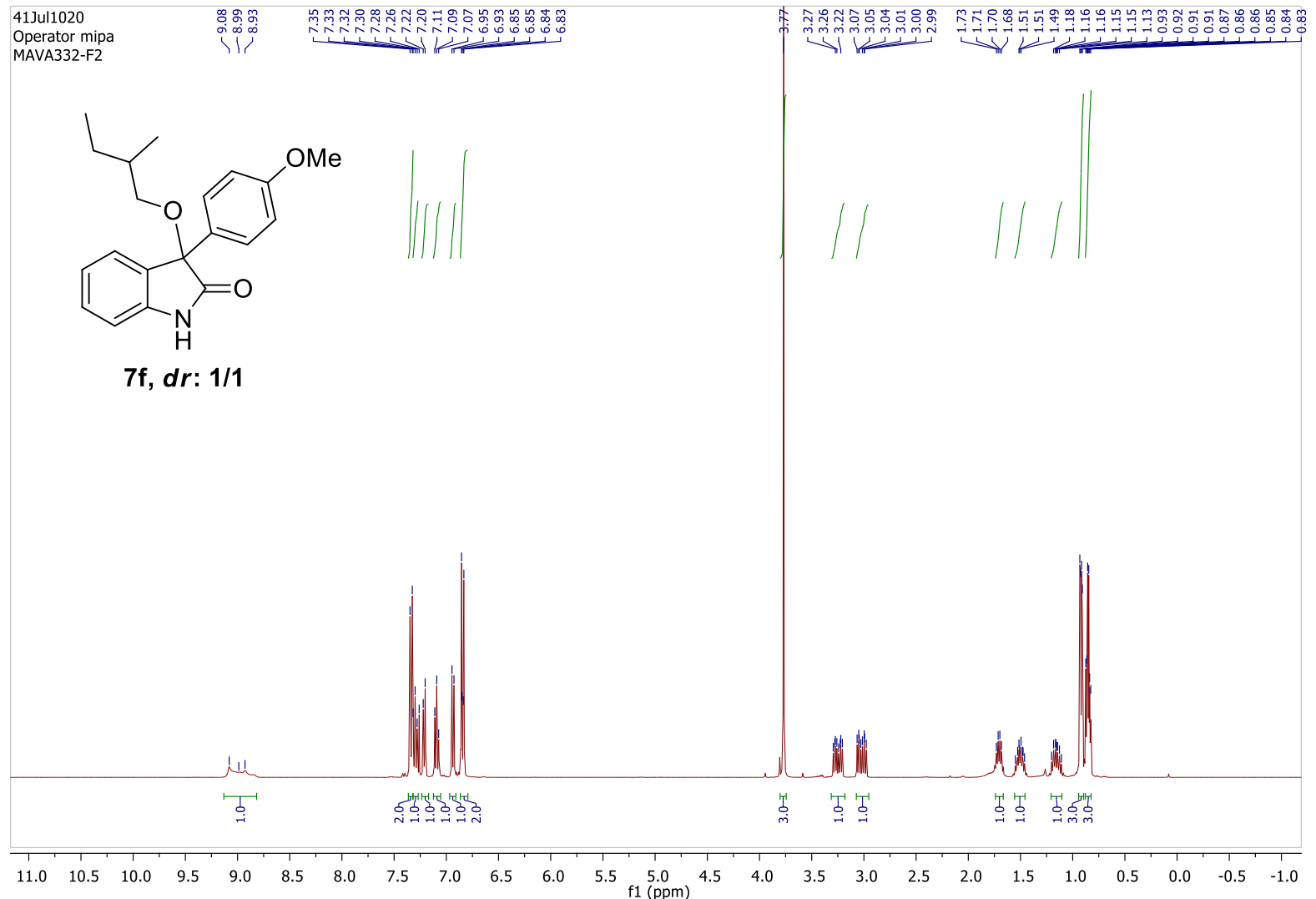

${ }^{1} \mathrm{H}$ NMR in $\mathrm{CDCl}_{3}$ at $400 \mathrm{MHz}$

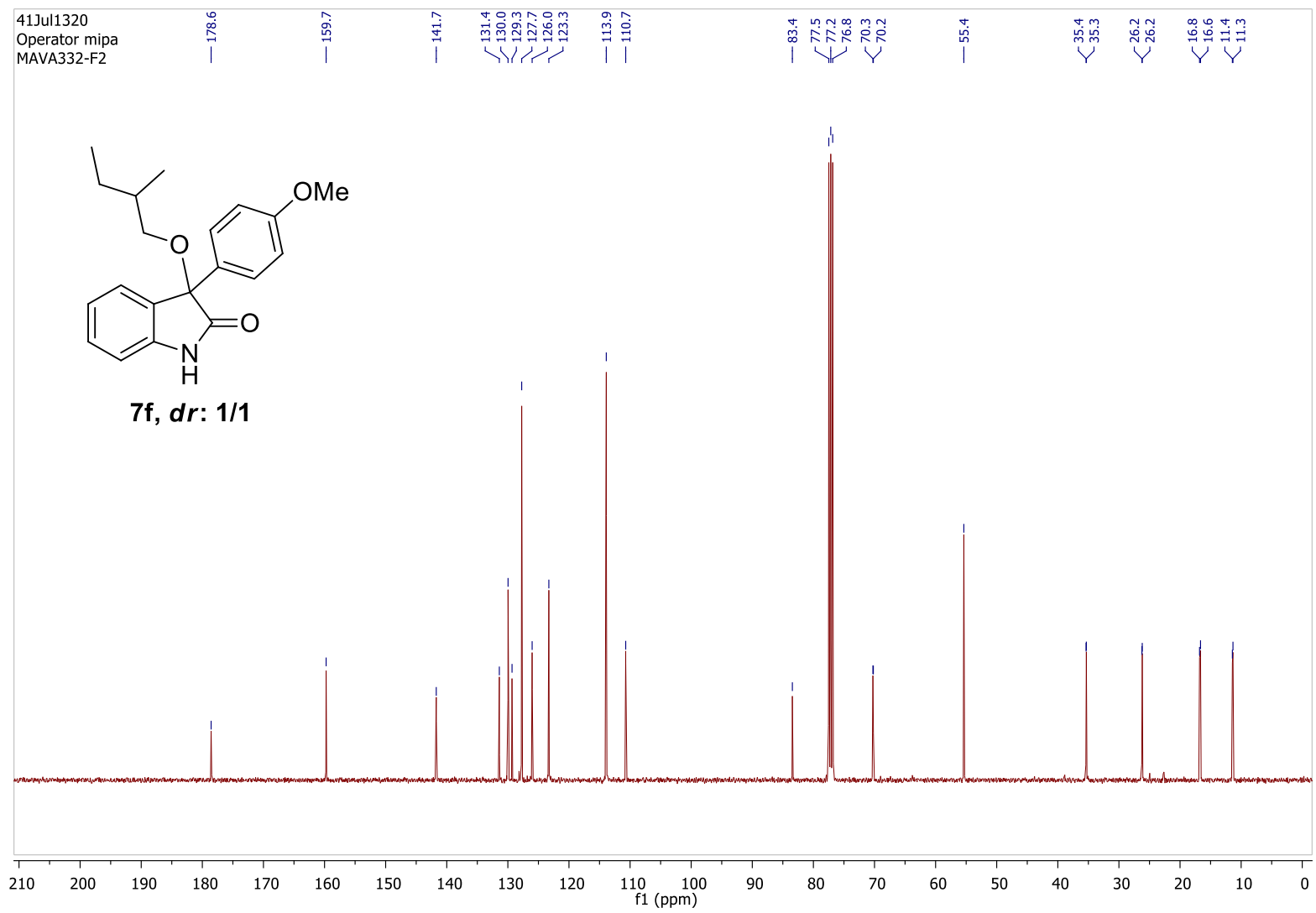

${ }^{13} \mathrm{C}\left\{{ }^{1} \mathrm{H}\right\} \mathrm{NMR}$ in $\mathrm{CDCl}_{3}$ at $100 \mathrm{MHz}$ 


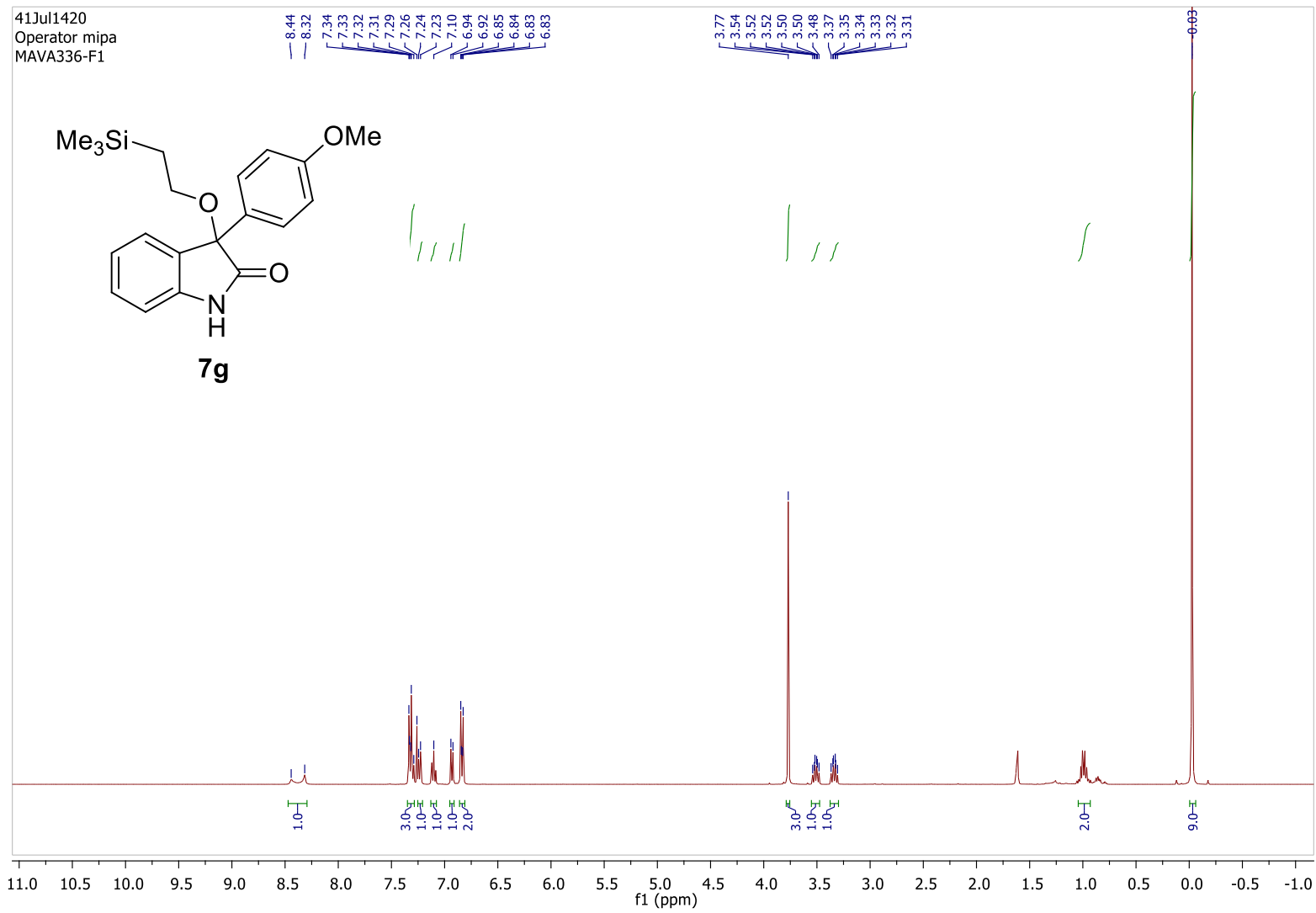

${ }^{1} \mathrm{H} \mathrm{NMR}$ in $\mathrm{CDCl}_{3}$ at $400 \mathrm{MHz}$

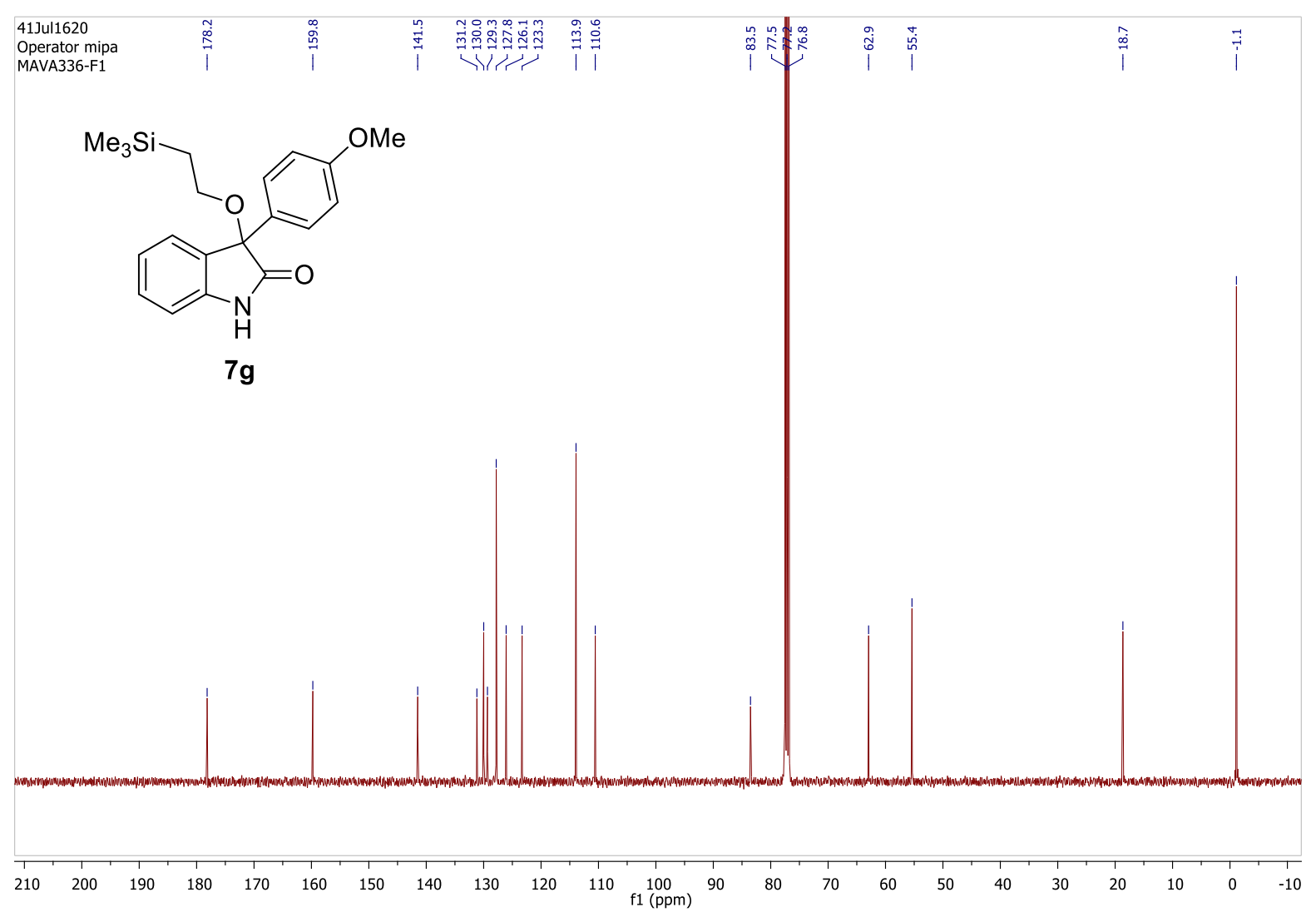

${ }^{13} \mathrm{C}\left\{{ }^{1} \mathrm{H}\right\} \mathrm{NMR}$ in $\mathrm{CDCl}_{3}$ at $100 \mathrm{MHz}$ 


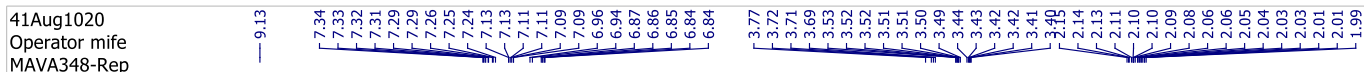

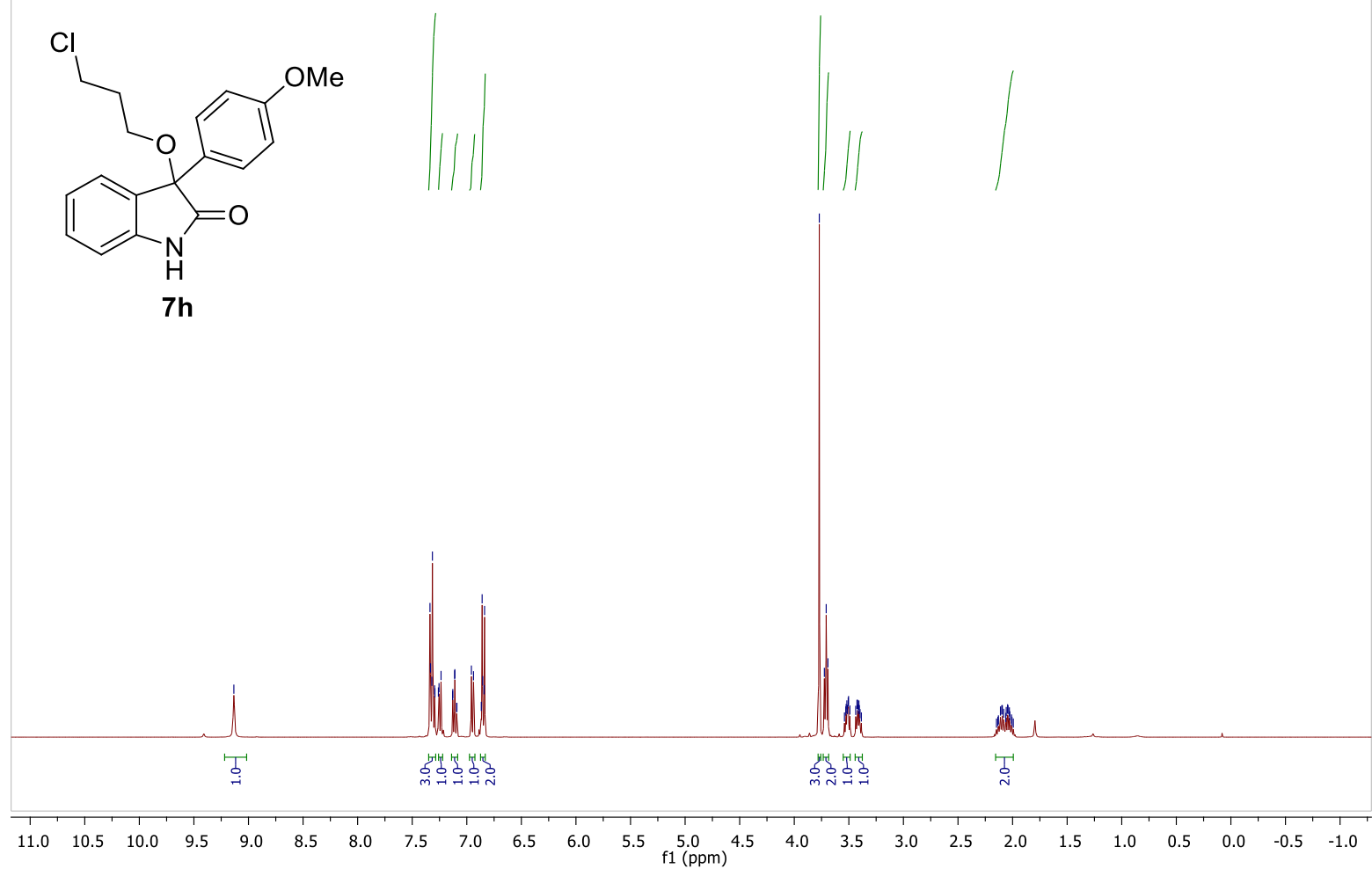

${ }^{1} \mathrm{H} \mathrm{NMR}$ in $\mathrm{CDCl}_{3}$ at $400 \mathrm{MHz}$

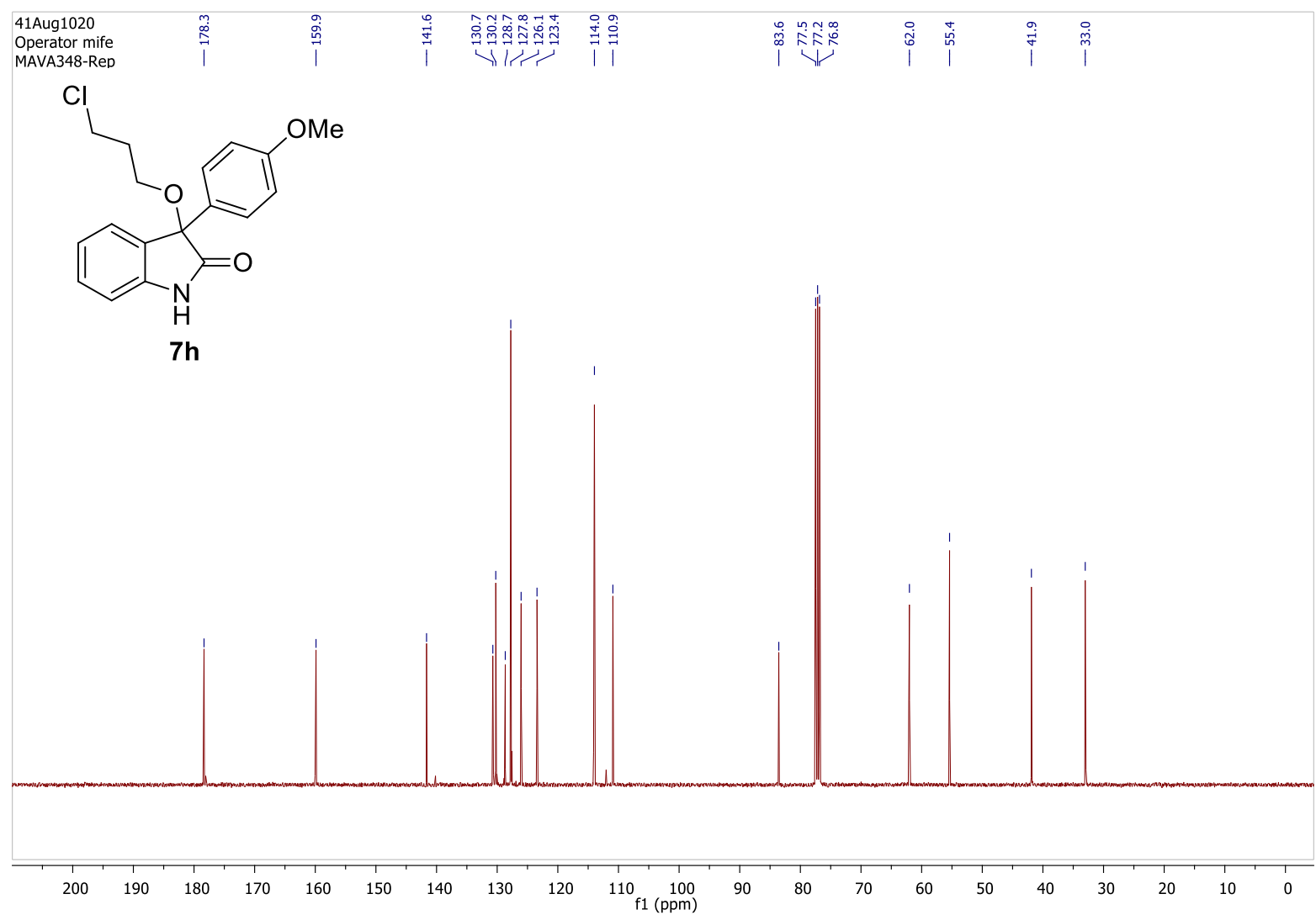

${ }^{13} \mathrm{C}\left\{{ }^{1} \mathrm{H}\right\} \mathrm{NMR}$ in $\mathrm{CDCl}_{3}$ at $100 \mathrm{MHz}$ 

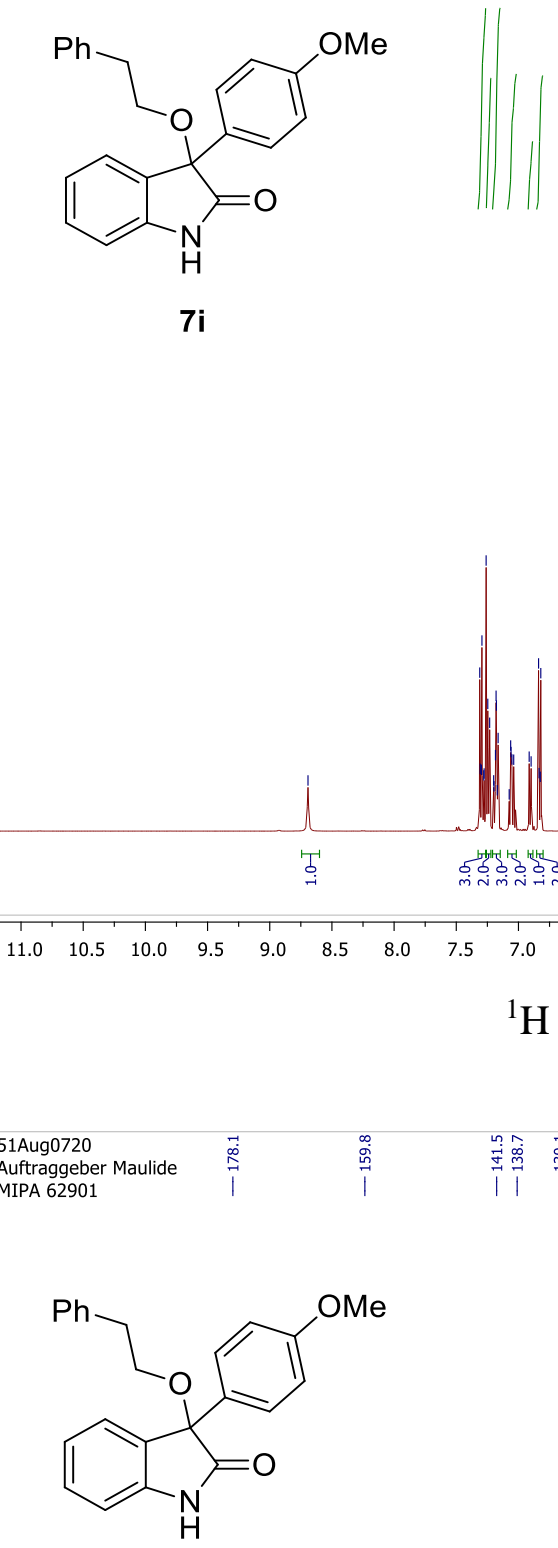

$7 \mathbf{i}$

${ }^{l} \mathrm{H} \mathrm{NMR}$ in $\mathrm{CDCl}_{3}$ at $500 \mathrm{MHz}$

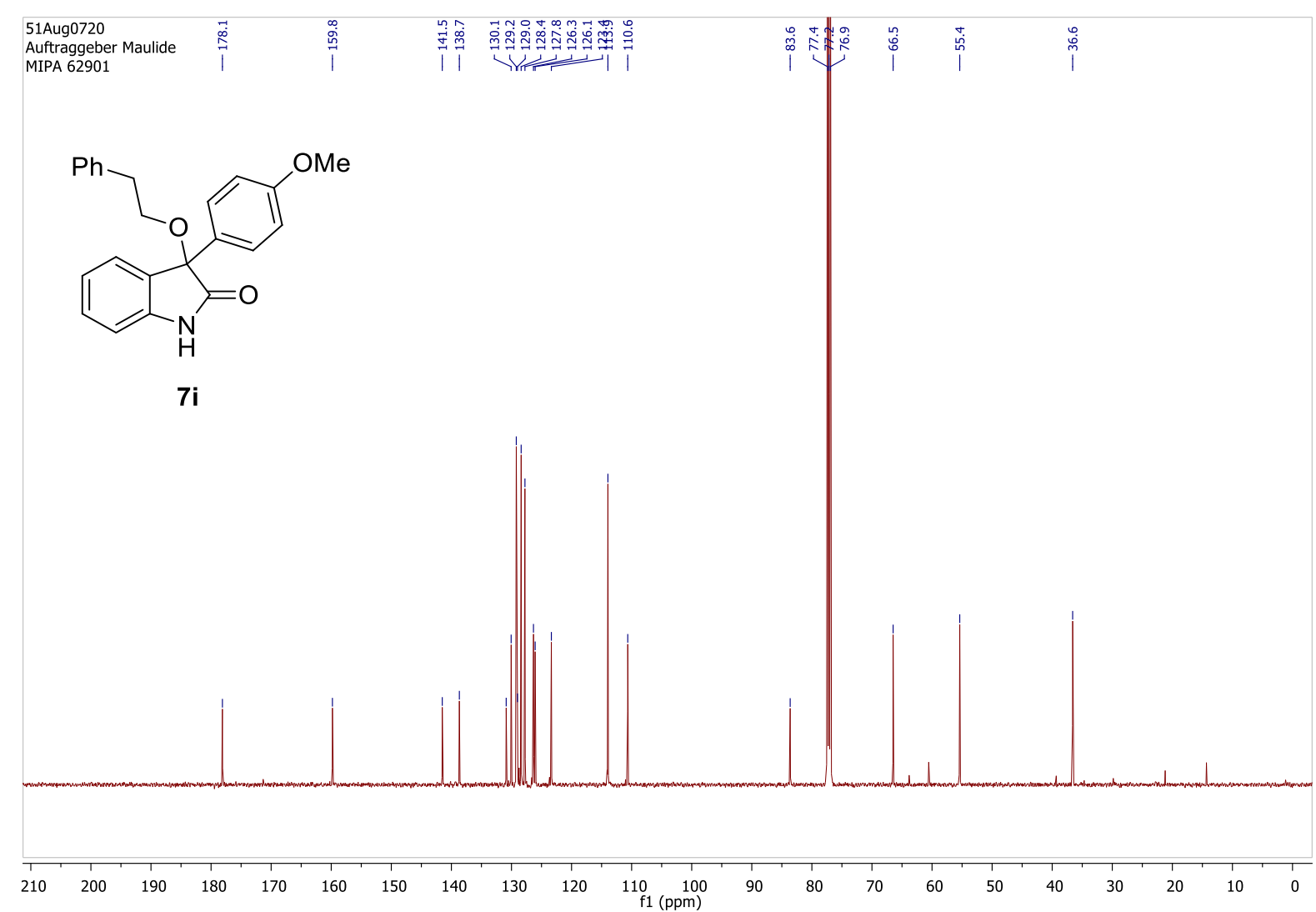

${ }^{13} \mathrm{C}\left\{{ }^{1} \mathrm{H}\right\} \mathrm{NMR}$ in $\mathrm{CDCl}_{3}$ at $126 \mathrm{MHz}$ 


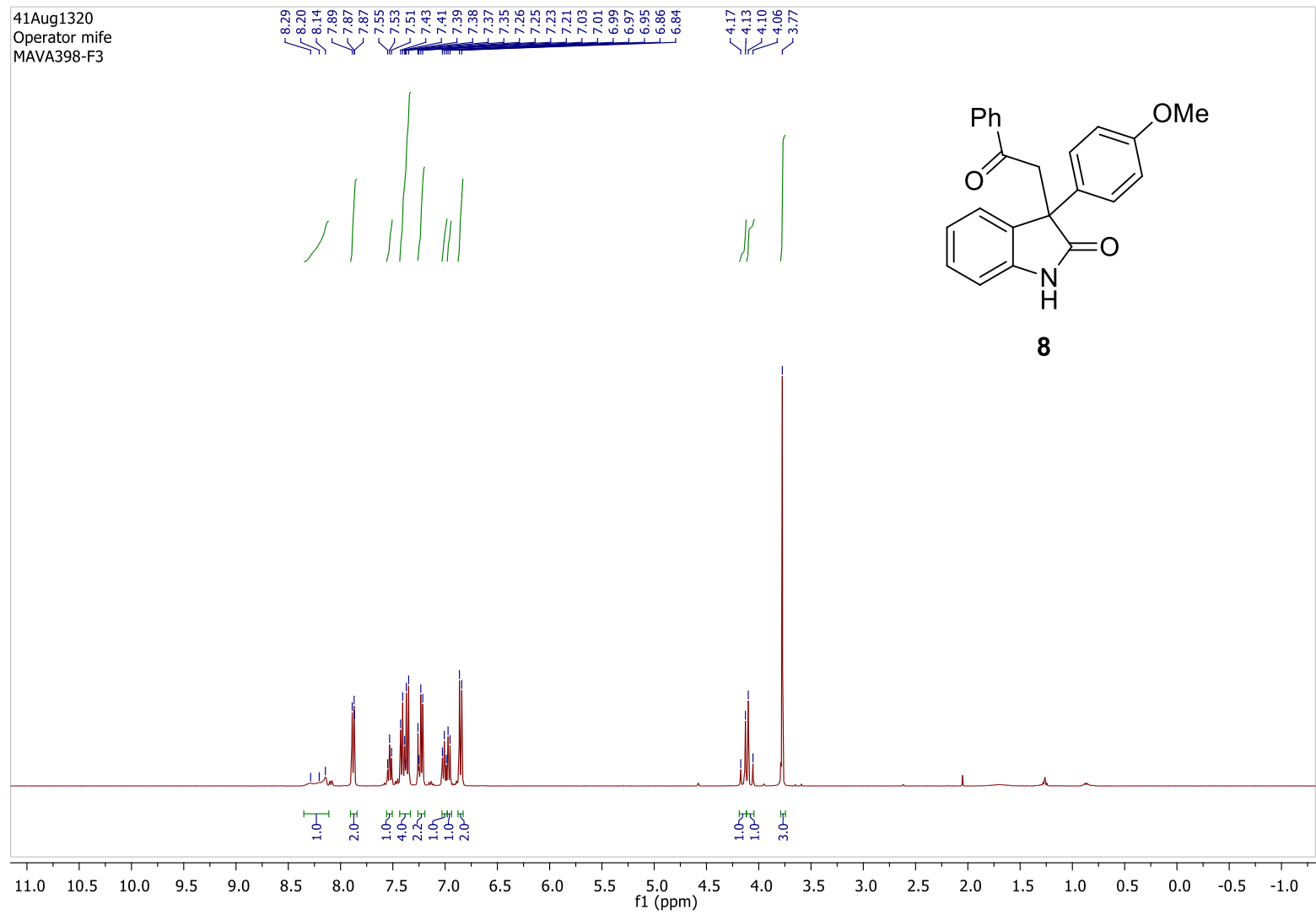

${ }^{1} \mathrm{H} \mathrm{NMR}$ in $\mathrm{CDCl}_{3}$ at $400 \mathrm{MHz}$

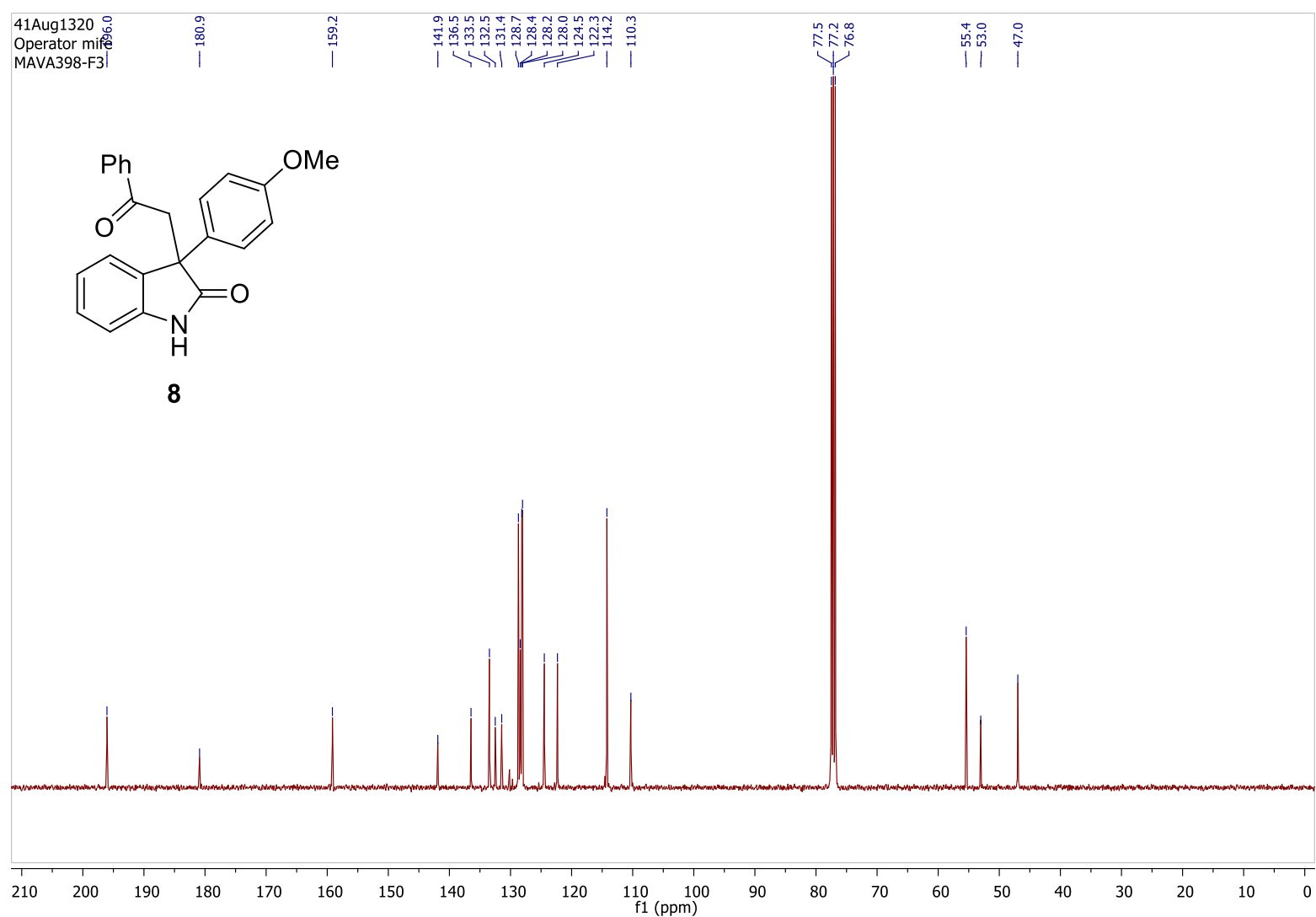

${ }^{13} \mathrm{C}\left\{{ }^{1} \mathrm{H}\right\} \mathrm{NMR}$ in $\mathrm{CDCl}_{3}$ at $100 \mathrm{MHz}$ 


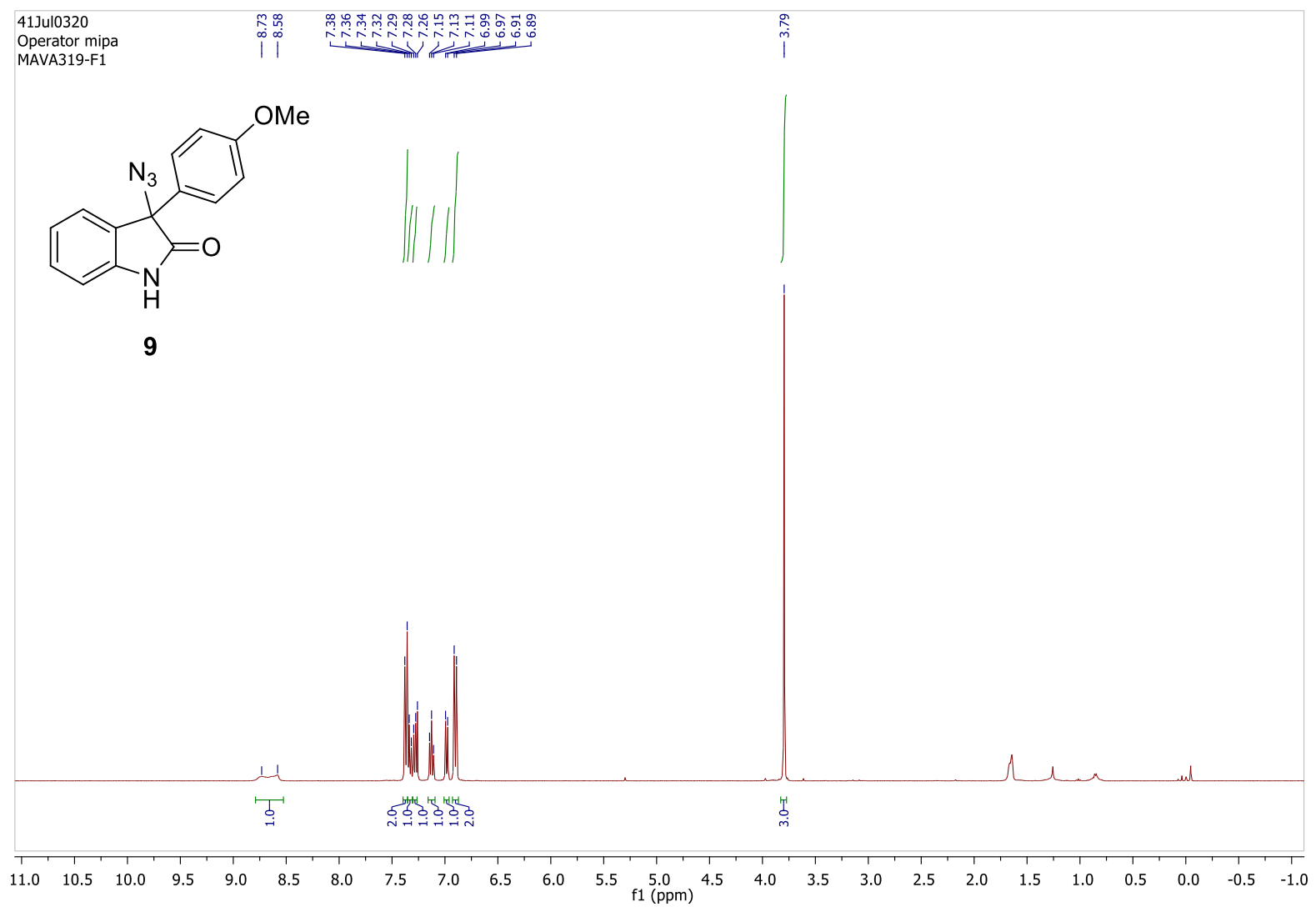

${ }^{1} \mathrm{H} \mathrm{NMR} \mathrm{in} \mathrm{CDCl}_{3}$ at $400 \mathrm{MHz}$

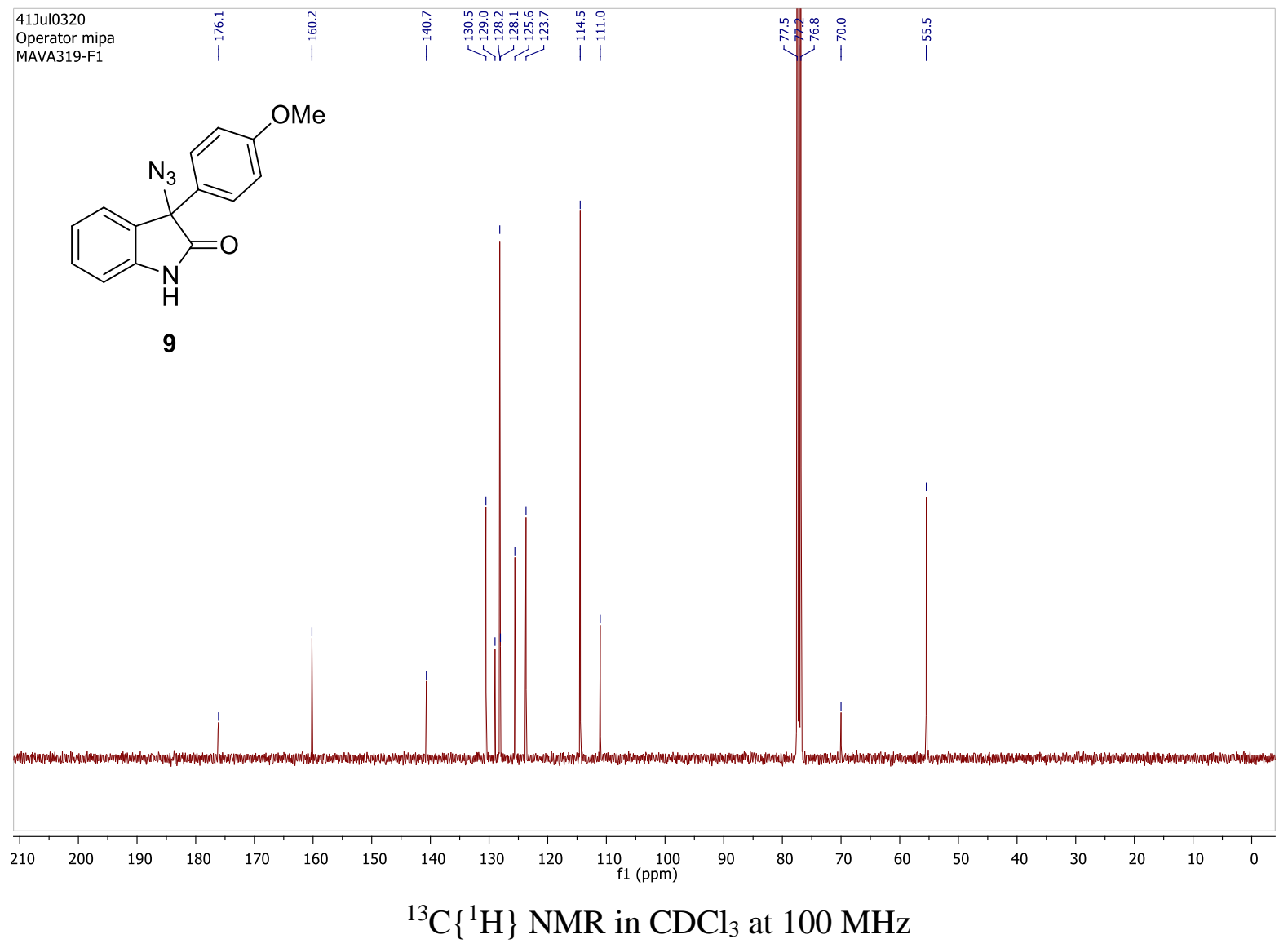




\section{References}

${ }^{1}$ Trost, B. M.; Zhang, Y. Mo-Catalyzed Regio-, Diastereo-, and Enantioselective Allylic Alkylation of 3-Aryloxindoles. J. Am. Chem. Soc. 2007, 129, 14548-14549.

2 Jensen, T.; Madsen, R. Ruthenium-Catalyzed Alkylation of Oxindole with Alcohols. J. Org. Chem. 2009, 74, 3990-3992.

${ }^{3}$ Trost, B. M.; Zhang, Y.; Zhang, T. Direct N-Carbamoylation of 3-Monosubstituted Oxindoles with Alkyl Imidazole Carboxylates. J. Org. Chem. 2009, 74, 5115-5117.

${ }^{4}$ Dua, T. -P.; Zhu, G. -G.; Zhou, J. A Facile Method for the Synthesis of 3-Alkyloxindole. Lett. Org. Chem. 2012, 9, 225-232.

${ }^{5}$ Xu, J.; Liang, L.; Zheng, H.; Chi, Y. R.; Tong, R. Green oxidation of indoles using halide catalysis. Nat. Commun 2019, 10, 4754.

${ }^{6}$ Cao, S. -H.; Zhang, X. -C.; Wei, Y.; Shi, M. Chemoselective Reduction of Isatin-Derived ElectronDeficient Alkenes Using Alkylphosphanes as Reduction Reagents. Eur. J. Org. Chem. 2011, 2668-2672.

${ }^{7}$ Altman, R. A.; Hyde, A. M.; Huang, X.; Buchwald, S. L. Orthogonal Pd- and Cu-Based Catalyst Systems for C- and N-Arylation of Oxindoles. J. Am. Chem. Soc. 2008, 130, 9613-9620.

${ }^{8}$ Toullec, P. Y.; Jagt, R. B. C.; de Vries, J. G.; Feringa, B. L.; Minnaard, A. J. Rhodium-Catalyzed Addition of Arylboronic Acids to Isatins: An Entry to Diversity in 3-Aryl-3-Hydroxyoxindoles. Org. Lett. 2006, 8, 2715-2718.

${ }^{9}$ Xiao, Z. -K.; Yin, H. -Y.; Shao, L. -X. N-Heterocyclic Carbene-Palladium(II)-1-Methylimidazole Complex Catalyzed $\alpha$-Arylation of Oxindoles with Aryl Chlorides and Aerobic Oxidation of the Products in a One-Pot Procedure. Org. Lett. 2013, 15, 1254-1257.

${ }^{10}$ Gade, A. B.; Bagle, P. N.; Shinde, P. S.; Bhardwaj, V.; Banerjee, S.; Chande, A.; Patil, N. T. Catalytic Enantioselective 1,3-Alkyl Shift in Alkyl Aryl Ethers: Efficient Synthesis of Optically Active 3,3'Diaryloxindoles. Angew. Chem. Int. Ed. 2018, 57, 5735-5739.

${ }^{11}$ Zhang, Z.; Smal, V.; Retailleau, P.; Voituriez, A.; Frison, G.; Marinetti, A.; Guinchard, X. Tethered Counterion-Directed Catalysis: Merging the Chiral Ion-Pairing and Bifunctional Ligand Strategies in Enantioselective Gold(I) Catalysis. J. Am. Chem. Soc. 2020, 142, 3797-3805.

${ }^{12}$ Xia, J. -T.; Hu, X. -P. Copper-Catalyzed Asymmetric Propargylic Alkylation with Oxindoles: Diastereo- and Enantioselective Construction of Vicinal Tertiary and All-Carbon Quaternary Stereocenters. Org. Lett. 2020, 22, 1102-1107.

${ }^{13}$ Polidano, K.; Allen, B. D. W.; Williams, J. M. J.; Morrill, L. C. Iron-Catalyzed Methylation Using the Borrowing Hydrogen Approach. ACS Catal. 2018, 8, 6440-6445.

${ }^{14}$ Wang, H. -L.; Li, Y. -M.; Wang, G. -W.; Zhang, H.; Yang, S. -D. Scandium(III) Triflate Catalyzed Direct Cyclization of Ketoamides for the Synthesis of 3-Hydroxy-2-Oxindoles. Asian J. Org. Chem. 2013, 2, 486-490.

${ }^{15}$ Liu, X. -L.; Yue, J.; Chen, S.; Liu, H. -H.; Yang, K. -M.; Feng, T. -T.; Zhou, Y. Thermal-mediated catalyst-free heterolytic cleavage of 3-halooxindoles: rapid access to 3-functionalized-2-oxindoles. $\mathrm{Org}$. Chem. Front. 2019, 6, 256-262.

${ }^{16}$ Hino, T.; Endo, M.; Tonozuka, M.; Hashimoto, Y.; Nakagawa, M. Bromination of 2-Bromo, 2Ethylthio, and 2-Ethylsulfonylindoles with N-Bromosuccinimide. Isolation and Reactions of 1Bromoindoles and 3-Bromoindolenines. Chem. Pharm. Bull. 1977, 25, 2350-2358.

${ }^{17}$ Escolano, C.; Vallverdú, L.; Jones, K. Reaction of Indolin-2-ones with Cerium(IV) Ammonium Nitrate. Tetrahedron 2002, 58, 9541-9545. 